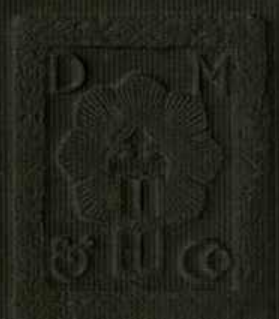




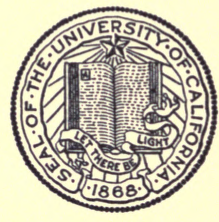

THE LIBRARY OF

THE UNIVERSITY OF CALIFORNIA

LOS ANGELES 
Dummay vale.

L.C. $\&$.

hanch' 26 . 





\section{ELEMENTS OF PLANT BIOLOGY}





\section{ELEMENTS OF PLANT BIOLOGY}

BY

A. G. TANSLEY, M.A., F.R.S.

University Lecturer in Botany and Examiner in Elementary

Biology to the University of Cambridge

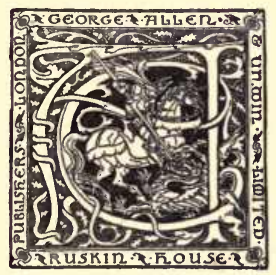

LONDON: GEORGE ALLEN \& UNWIN LTD. RUSKIN HOUSE, 4O MUSEUM STREET, W.C. I NEW YORK : DODD, MEAD \& COMPANY 
(All rights reserved)

Printed in Greal Briteint 


\section{Biomed \\ QK \\ 711 \\ T159e \\ 1922}

\section{PREFACE}

THIs book is intended primarily for medical students and others who do not necessarily intend to continue the study of botany, but who desire or are obliged to obtain some elementary knowledge of plants, particularly in relation to general biology. It may perhaps also be useful in the highest forms of schools where biology is taught, as well as in training colleges. But it is unsuitable for pupils under seventeen.

The book is based on the first portion, which deals mainly with plants, of the course in Elementary Biology for the Preliminary Examination in Science and the First Examination for the M.B. degree at Cambridge. This part of the course has been framed, in complete freedom as to the material chosen and its treatment, but in co-operation with the Professor of Zoology, to serve as an introduction to Biology suitable for freshmen, many of whom know nothing whatever of the subject. This first portion of the course, which is covered by the present work, occupies one term, and comprises twenty-four lectures and forty-eight hours of practical work in the laboratory. The rest of the course, which deals mainly with animals, comprises about thirty-six lectures and seventy-two hours of practical work, and includes some elementary treatment of heredity and evolution.

No claim is made to ideal pedagogic method. The problem we are faced with at Cambridge is a strictly 
practical one, conditioned by the fact that the students include some who know nothing of biology and are only beginning to learn elementary chemistry and physics, and by the further fact that the classes are large, varying from 120 to $25^{\circ}$ students, and have to be taught on the old-fashioned academic system of an hour's lecture followed by two hours' practical work, a system which has serious educational drawbacks, though also obvious conveniences.

The problem, then, is to provide, under these conditions, a course of teaching which shall be as interesting as possible and shall serve to introduce the student to the fundamental facts and principles of biology, both as part of his training for life and more particularly as an introduction to the study of medicine, which, as has often been said, is really a specialised branch of applied biology.

The time is past when we could hope to give the budding medical student a thorough training in elementary botany and zoology, and with the ever increasing complexity of the medical curriculum proper there is constant pressure to shorten and simplify the preparatory parts. It seems to me that biologists should yield to this pressure to a reasonable extent, and frankly ask themselves how far the things that have been traditionally taught really serve as the best introduction to biology for medical students under existing conditions.

At the same time the provision of a good introduction to general biology in the early part of the student's course is certainly of as much importance now as ever it was. If the foundations are not broad enough, the student's outlook will necessarily suffer when he comes to his more specialised work; and 
some serious knowledge of the specific structures, activities and evolution of plants is an essential part of a broad biological foundation. There are things about life as a whole that we can only learn from plants, and there are others which some knowledge of plants helps us to understand far more completely. The ideal course in elementary biology would undoubtedly be given by a teacher dealing with both plants and animals. Unfortunately, the differentiation of botany and zoology has gone much too far to make it possible to find or to employ such a teacher in most existing British universities. The Cambridge course attempts a compromise which approaches, though it cannot attain, this ideal, for the introductory lectures deal with animals as well as with plants, while plants as well as animals are freely used as illustrations in the concluding lectures of the zoological portion, which are concerned with heredity and evolution.

If all school children were taught some biology, as they certainly should be, and this were on well considered and recognised lines, the task of the university teacher would be more straightforward and more profitable: he could give his students a far better training in the time at his disposal. As it is he is bound to assume that they know nothing of biology and very little of chemistry and physics. A rudimentary knowledge of inorganic chemistry is, however, assumed in this book, and this is now fairly common among students entering the university.

The chief points in which this book differs from most works of similar scope are the following. First of all much more space is devoted to biological facts 
of general significance which can be best illustrated by the lower forms of plant life. The vascular plants are briefly treated: an attempt is made to outline the structure and life history of the seed plant, but much of the morphology is left on one side as of no great interest or importance to the student of general biology. Secondly, after an introductory lecture on the general characters and differences of animals and plants, the student is at once introduced to the most important organic substances which make up the body of the organism, and then to a brief consideration of some of the physical characters of organic substances and of protoplasm. This is followed by an account of amœba, and of the chief functions of organisms in general, and this again by a general account of the cell (Chapter VI). This line of approach, entered upon before the student knows anything worth mentioning of the detailed structure of organisms, certainly has drawbacks, but in a necessarily short course it has also great advantages, because it enables the structures and activities described later on to be followed with some understanding of their significance in the light of the funda. mental properties and functions of organic and living substance.

The Green Plant cell, as the most fundamental unit in the plant world, is dealt with in Chapter VII, and then the plant cell without chlorophyll, illustrated (as functioning organisms) by Yeast and Bacteria. The Bacteria are considered at greater length than is usual in elementary books of this scope, because of their enormous importance in the general economy of the world, and because, as it seems to the author, it is specially desirable for medical students to get 
a fair general notion of their activities before they enter, later in their medical course, upon the highly specialised study of the disease-producing formswhich are, after all, from the general biological standpoint, a relatively unimportant fraction of the group. The saprophytic and parasitic fungi are next dealt with, and then the more detailed study of the chlorophyll-bearing plants is entered upon, and occupies the rest of the book. Here the evolutionary series is roughly followed, for it seems to the author that the interest and educational advantage of this method of treatment are decisively in its favour.

The Chlamydomonas-Volvox series is dealt with in much more detail than is usual, because of the unique interest of this series as illustrating the origin of sex and of the soma. Fucus is taken as the best example of primitive tissue differentiation in a relatively large plant, Pellia as a simple land plant. The archegoniate plants are treated very briefly indeed, detailed descriptions of structure and development being deliberately omitted, though an attempt is made to give, in very brief outline, the fundamental facts of the biological significance of vascular plants and of heterospory.

The rest of the book deals with the Seed Plants, first with their external form and methods of vegetative propagation, then with the architectural elements of which they are composed-a course which has been found very useful in practical teaching because it relieves the later descriptions of the structure of the plant organs from being overloaded with accounts of the tissue elements themselves. The vegetative organs of the plant-root, leaf and stem-follow, and the account of the vegetative structure of the 
seed plant closes with the woody stem. Finally, the flower, fruit, seed and seedling are dealt with in order, thus completing the sketch of the structure, economy and life history of the seed plant.

Throughout the book the effort has been made to treat the material from the point of view of general biology rather than with the narrower outlook of pure botany.

The schedules of practical work follow pretty closely those in use in the Cambridge course; in a few cases alternative material is suggested. Though it is not suggested that the selection of material cannot be improved, nor to be supposed that any teacher will desire to follow the schedules in every detail, it was thought that the interests of teachers and students would best be served by presenting a perfectly definite selection of material which has been proved by experience to be workable and instructive. Section B on p. 34 and (7) on p. 47 were suggested to me by Dr. M. C. Rayner.

The practical work suggested at the end of each chapter occupies from 2 to $2 \frac{1}{2}$ hours. It is not contemplated that the students should cut their own sections in that time. It is found by experience that so large a proportion of the time available in a short course is spent in learning to cut sections, and the sections cut are often so inferior, that the result is wholly unsatisfactory. Much better results are obtained by providing students with good sections and then insisting that they shall examine and draw them properly. It is not of course suggested that it is not good for students to do as much of the necessary manipulation of their material as possible, but the particular technique of cutting hand-sections 
with a razor is of no further use to anyone unless he becomes a professional botanist (of no very great use indeed to many professional botanists), and to acquire any useful proficiency occupies far more time than it is worth, when the student's hours are strictly limited.

With a small class and ample time the case is quite otherwise, and not only should students cut many of their own sections under these conditions, but they may be taught to prepare material and experiments with great advantage to themselves and to their ultimate grip of the subject. Such conditions, however, simply do not exist in big university classes with every hour of the student's working day filled up.

In the earlier schedules, especially, fairly precise instructions are given to the student, and some general hints are prefixed to the book (p. I 7$)$, suggested by experience of the mistakes students most frequently make in doing their practical work. They are of course intended only for those who have no previous experience of microscopic work in the laboratory.

It is believed that the actual measurement of microscopic objects, though not usual in elementary classes, is a valuable help to the student in relating the structures he sees under the microscope to the objects he sees with the naked eye, giving the former a greater objective reality in his mind. It is therefore suggested that the student should learn early in the course to calibrate a micrometer eyepiece with a micrometer slide, and that he should frequently measure the diameters of microscopic objects.

I am greatly indebted to my daughter Margaret, who has drawn the whole of the illustrations. The 
majority are copied, with or without modification, from published figures. They are intentionally limited in number, no attempt having been made to illustrate everything that is described when the details of structure can quite easily be made out on the objects seen in the laboratory. Accurate pictures of the material actually studied provide too great a temptation to copy the picture rather than the object, and it is, of course, impossible to lay too great stress on the value of making careful drawings from the objects themselves. For this reason a good deal of the structure of the higher plants is illustrated by diagrams and generalised pictures which are not faithful copies of nature.

Professor A. E. Boycott, of University College, London, has been good enough to read the chapter on Bacteria, and has given me the help of his very valuable criticisms. My colleague, Mr. F. T. Brooks, has kindly performed a similar service in reading Chapters $\mathrm{X}$ and $\mathrm{XI}$, on the Fungi. Mr. S. M. Wadham, the Senior Demonstrator in Botany at Cambridge, has given ungrudging help in organising the Practical Work and suggesting improvements. Finally, I am deeply indebted to my friend Dr. F. F. Blackman, who has placed at my disposal his critical knowledge of plant physiology and biochemistry by advising in detail on Chapters II, III and VII, as well as on other smaller sections of the book.

A. G. T.

CAmbridge, July, 1922. 


\section{CONTENTS}

PAGE

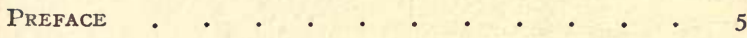

Hints to Students on Practical Work • • • I7 CHAPTER

I. Introductory. Plants and Animals • 2 I

II. Organic Substances and their Chemical

Characters . . . . . . . . 36

III. Some Physical Characters of Organic SubSTANCES • . . . • . . . 48

IV. Protoplasm and the Amcba. Protococcus - $6 \mathrm{I}$

V. The Vital Functions • • • • • 76

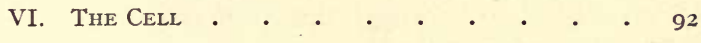

ViI. The Green Plant Cell . • • • • $\quad$ II 2

ViII. The Colourless Plant Cell. The Yeast

Plant • • • 1 • • • • • • $~ I 27$

IX. Bacteria • • • • • • • • $~ 138$

X. Saprophytic Fungi. Mucor and Penicillium. I57

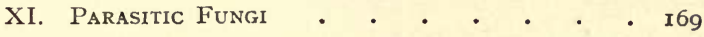

XiI. Origin of Sex and of the Soma. The

Green Algex

XiII. Differentiation of Tissues. Fucus: The Sea Wrack

$$
15
$$


XIV. The Simplest Land Plants-Liverworts and Mosses. The Pteridophyta . . 23I

XV. The Seed Plants: Forms and Life Histories 254 XVi. The Tissue Elements of Seed Plants . . 270 XVII. The Root . . . . . . . . . 288 XViII. The Foliage Leaf . . . . . . 302 XiX. The Primary Stem . . . . . . . 315

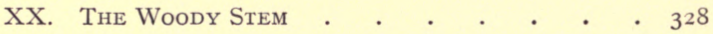

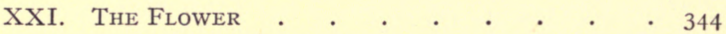
XXII. The Fruit . . . . . . . . $36 \mathrm{I}$ XXiII. The Seed and its Germination . $\quad$ • 375

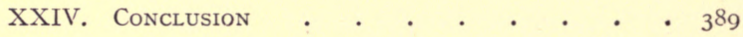
INDEX $\quad . \quad \cdot \quad \cdot \quad \cdot \quad \cdot \quad \cdot \quad \cdot \quad \cdot 405$ 


\section{HINTS TO STUDENTS ON PRACTICAL WORK}

\section{Use of the Microscope.}

(I) Remember that the microscope is a delicately adjusted scientific instrument, and must not be treated roughly. Do not lift it by the tube, but always by the solid part of the stand.

(2) Take great care not to get liquids of any kind on to the lenses. If a lens is dusty, wipe it with a soft handkerchief.

(3) Always see that the slide and coverslip are perfectly clean before use.

(4) If you cannot then get a clear view of an object, look first to the surfaces of the lenses, especially of the objectives. If there is glycerine on the surface of the lens, it must be carefully and repeatedly wiped with a soft handkerchief. Breathing on the lens between wipings will often help. Take care never to scratch the lenses. This is often done by rubbing with a harsh cloth.

(5) In examining an object or section under the microscope look at it first with the naked eye, then with a hand lens, then with the low power, and finally with the high power. This procedure enables you to get a preliminary idea of the general form of the object or section, before you look at its details, and thus to relate the details to the larger features of its structure. Remember that the world you see under the microscope is not really a different world from that which 
you see with the naked eye. The microscope is merely an instrument which extends the power of the eye so that it can detect the fine details of structure.

(6) Never put on the high power unless the object is covered with a coverslip, or you will be almost certain to get the mounting medium on to the lens of the objective.

(7) Never focus the high power carelessly, or you will jam it on to the slide, spoil the object, and very likely injure the objective. When you have put on the high power, note its distance from the coverslip by looking from the side (you will soon learn to judge about the distance at which it is in focus). Correct the distance with the coarse adjustment carefully, till you judge it is about right, and then look through the microscope and focus accurately with the fine adjustment.

\section{Observation and Drawing of Structure.}

Careful observation, followed by accurate drawing of the structures of plants and animals, whether seen with the naked eye, with a hand lens or under the microscope, is an indispensable means of acquiring a sound first-hand knowledge of biology. It is also one of the best methods of training the mind to form and retain clear mental pictures, and this power is most valuable in almost every occupation of life, most notably in the everyday work of the medical man. It is therefore of great importance that the student should seriously bend his mind from the start to learning how to represent what he sees in drawings and diagrams.

The power of making good representations on paper of what one sees varies very much in different people -some find it comparatively easy, others very difficult. 
But those students who find it difficult should not be discouraged, for everyone can improve this power by practice, and it is the effort to improve which is specially valuable as training of the mind.

The following points should always be kept in view :-

(I) Outlines should be represented by clear firm lines on smooth paper with a pencil of medium hardness sharpened to a good point. Avoid all "muzziness" in drawings: it always indicates a " muzzy" mental picture, which is useless. Do not put in "shading" in drawings of microscopic objects, and never "shade" any drawing till you are quite satisfied that the outlines are accurate. Shading is very seldom necessary.

(2) Draw only what you can actually see. Never pretend to see what you do not see. But remember that practice in observation will enable you to see things that you cannot see at first. So do not jump to the conclusion that things cannot be seen which the demonstrator says can be seen, because you cannot see them at once.

(3) Always draw on a large scale, so that all details can be clearly shown.

(4) Always write the names of the parts of what you draw at the side of the drawing, either in full or with unmistakable abbreviations, connecting each name with the corresponding part by a straight line, so that the drawing is at once intelligible.

(5) When you have to represent a complicated structure as seen under the microscope, for instance a section of an organ of a higher plant showing various tissues, make (a) a diagram, including the outlines only of the tissues, under the low power, to show the distribution of the tissues; and (b) detailed drawings 
20 HINTS TO STUDENTS ON PRACTICAL WORK

of small samples of each tissue under the high power, to show the structure of the different cells.

Do not make a drawing of the whole section and try to put in all the cells. To do it accurately would take a very long time, and the time spent would be largely wasted. To do it carelessly and inaccurately is worse than waste of time: it is training in slovenly work. 


\title{
Elements of Plant Biology
}

\author{
CHAPTER I
}

\section{INTRODUCTORY. PLANTS AND ANIMALS}

BotAny is that part of biology specially concerned with those living organisms we call plants. Plants form the basis of all life as it is lived upon the earth, because they alone have the power of making new supplies of what are called organic substances-the only substances on which animals can feed-out of inorganic substances. And the study of plants teaches us some things about life which we cannot learn, and others which we cannot so easily learn, from the study of animals. It is therefore necessary not only for all those who want to acquire some knowledge of biology as a whole, but also for those who will be daily concerned in their profession with the most highly developed and most complex of all living organisms - man-to learn something about plants if they are to acquire a firm foundation for their later studies. It is not necessary for them to gain the detailed and comprehensive knowledge that the botanist has to acquire, and their studies should be directed rather to what plants can teach them about life as a whole than to a knowledge of plants for their own sakes. The title of this book, which is designed especially for the medical student who knows nothing of biology, 
and whose time is strictly limited, is intended to emphasise this point of view. But such a student must understand that if he is to acquire a foundation which shall be of real use to him in his later work he must give himself freely to the labour of acquiring a firm elementary knowledge of the nature and constitution of the bodies of plants, of the outline of their structures and of their various activities, not only from books and lectures, but also at first-hand in the laboratory.

\section{What is a Plant? Differences between Animals and} Plants.-The animals and plants with which we are most familiar are the higher, i.e. the more complex ones. They mostly live on the land, and are on the whole, though by no means invariably, bigger than the lower, less complex, forms. Certain outstanding differences between the familiar higher animals, on the one hand, and the familiar higher plants, on the other, are very obvious. The animals move about, the plants are rooted in one place: the animals are compact, the plants are branching, in their habit of body: the animals are variously coloured, the plants are green, or at least have green leaves. The most important functional difference is that animals consume solid food, while plants do not; and this is really the root of all the other differences.

The locomotion of animals is related to the fact that they have to move about to find their food, which must be organic, i.e. must consist of special complex chemical substances forming part of, or produced by, other animals or plants, while the food of plants consists of liquid and gaseous inorganic substances which are found everywhere in the earth and air.

The compact form of animals is connected with the necessity of locomotion and with the fact that the 
body is organised round the gut (alimentary canal), which digests the organic food, i.e. changes it into forms which can directly nourish the body. The branching habit of plants, on the other hand, exposes the greatest possible surface to the soil and to the air, from which the plant directly absorbs the liquid and gaseous inorganic substances which form its food.

Plants are green or have green parts because of the green colouring matter (chlorophyll $\mathrm{x}$ ) which enables them to build up their bodies from simple inorganic substances-or, in other words, to form organic substances from inorganic.

And the many other differences of structure and organisation which distinguish the higher animals and the higher plants are all related, directly or indirectly, to the fundamental difference in their foods.

There are, however, cases in which both animals and plants depart, in one or other respect, from these general characters. There are parasitic animals which do not move about, but remain fixed on or in the " host," and which consume only liquid food, though this is always organic, and derived from the body of the host. There are plants which are partly carnivorous, with special organs which digest solid animal food and absorb the products of digestion. There are branching animals and compact plants. There are plants which live largely on organic (liquid) food.

Furthermore, there are simple minute microscopic organisms living in water and other liquids which have some animal and some plant characters, so that biologists have sometimes considered them as animals and sometimes as plants. These last are more or less changed representatives of some of the organisms

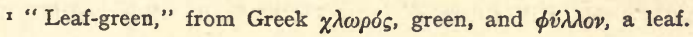


which were produced early in the history of life, and they have not become differentiated like the higher organisms, the unmistakable familiar animals and plants. Thus there is a minute oval organism called Chlamydomonas (Fig. 20, p. I85) which lives in pools. It is green and lives exclusively on liquid and gaseous food like a plant, but during most of its life it swims actively about like an aquatic animal. It must be reckoned as a plant, because, as we saw, the mode of feeding is the basal character of difference, but it still retains the animal character of free locomotion. And there are other minute organisms which feed in both ways, partly on liquid and gaseous inorganic food, partly on solid organic food, and these escape the meshes of any definition of an animal or of a plant.

We cannot, in fact, frame any comprehensive definition which shall sharply separate all living organisms into animals and plants. This conclusion illustrates a truth that the student will realise more and more fully as his biological studies progress, namely, that it is useless to expect the facts of nature exactly to fit our definitions, however carefully framed. There are so many different ways in which living substances may take shape, so many varieties of function, i.e. of ways in which it may work, and so many possible combinations of both of these, that there are certain to be exceptions to every rule we try to lay down. 'But that does not mean that we have to give up the task of analysing and classifying form and function, or the science of biology would be impossible. We can always recognise types of structure and types of function to which organisms conform, more or less closely, because their physical and chemical constitution forces them, so to speak, along certain lines of differentiation and behaviour. 
Only we must not expect to draw sharp lines. The forms included in any groups we make will always tend to shade off into forms belonging to other groups.

Thus we can say that the essential animal character depends upon the habit of consuming solid organic food; that the essential plant character depends upon the habit of absorbing liquid and gaseous inorganic food; that a man is indubitably an animal, while an oak tree is as indubitably a plant; that with the man we can group a host of other different kinds of organism as animals, and with the oak tree a host of other organisms as plants; but that when we come to minute microscopic organisms we find the differences which are so clear and sharp among the higher forms becoming bluried, till finally we arrive at forms of which it is impossible to assert that they are definitely animals or definitely plants.

Relation of Animals and Plants to Energy.-The essential difference between animals and plants in their relation to food determines also their characteristic difference in relation to energy. All living organisms may be regarded as machines transforming energy from one form into another, for instance, from the potential energy locked up in the molecules of organic food to the kinetic energy seen in motion of the body and in the production of heat (animal), or from the "radiant" (kinetic) energy of sunlight to the potential energy of organic substances formed in the body (green plant). This subject will be dealt with more in detail in later chapters.

Meanwhile we note the broad difference between animals and plants in their relation to energy is that animals consume organic food and spend the energy it contains in heat and motion, while plants build organic 
substance and store energy. And since life consists in the expenditure of energy, animals live much more intensely than plants, and correspondingly they are sensitive to much more varied stimuli, i.e. to the influences which lead to their expending energy in definite ways. But because they are alive plants do spend energy and are sensitive to stimuli. In the course of the life of a tree, for instance, a large amount of energy is expended in lifting the branches high into the air and in pushing the root tips through the soil, though these things are done so slowly, measured by the standard of the rate of motion in animals, that we are not at once impressed by them. The shoots of a plant also bend towards the light, and the roots towards supplies of water, i.e. they respond to different stimuli in definite ways. Also animals do store energy in various forms of organic food, e.g. glycogen (a form of starch) in the liver and the muscles, and thus have a reserve supply of potential energy which enables them to carry on their active life for a time without consuming fresh food.

\section{The Naming and Classification of Plants and Animals.}

-The immense multitude of individual plants and animals existing on the earth are exceedingly varied in form and structure, and in order to get any understanding of plant or animal life we must classify them in some way. We recognise at once-mankind has recognised from the earliest times-that there are many different kinds, but of these some resemble each other so closely that they are difficult to distinguish, and can only be separated by those who have made a special study of the forms in question, while others are very distinct indeed. For instance, a blackberry is obviously different from a raspberry in the colour and taste of the fruit, and in the fact that the former 
has hard prickles which can tear the flesh, while the latter has soft prickles which cannot. But there are many different kinds of blackberry, some of which differ from one another in such small and variable characters that even specialists who have spent a large part of their lives in the study of the blackberries do not agree as to exactly how they should be grouped.

We distinguish the kinds or groups of individuals which resemble one another more or less closely, and which interbreed freely, as species, but authorities differ as to what they consider species, and there is not yet agreement as to whether the conception of a species can be made at all a precise conception. Species which are most like each other are grouped into genera, and genera which are most like each other into families. Thus the raspberry and the blackberry are different species of one genus, the sweet briar and the dog rose of another genus belonging to the same family. The hare and the rabbit are different species of one genus; the dog, the wolf and the fox of another belonging to quite a different family, though to the same large group, the mammals, which include all animals that suckle their young, comprising such diverse types as rats and mice, elephants, whales and men.

The conventional nomenclature, which is used in naming the different species we recognise, and enables us to record and systematise them, gives each genus a Latin name-a noun-and adds a qualifying adjective for the species. Thus the genus to which both the blackberry and raspberry belong is called Rubus, the former (if we lump all the blackberries together as one species) being Rubus fruticosus, the latter Rubus idaus. The genus Rosa includes Rosa rubiginosa, the sweet briar, and Rosa canina, the dog rose, as well 
as many other species. Both these genera, along with many others, belong to one family, the Rosacea, or Rose family, because they have certain characters of the flower and fruit in common. The hare is Lepus timidus, the rabbit Lepus caniculus: the dog, the European wolf and the fox respectively, Canis familiaris, C. lupus, and C. vulpes. In the last two cases it will be seen that the specific name is a noun (the Latin name of the animal in question), though it is used in place of an adjective. Very many species, of course, have no common names in any language because they have not impressed their existence on man by their usefulness or harmfulness, or conspicuousness-they have not attracted his attention in any way, until botanists and zoologists began to study the different forms for their own sakes. The Latin nomenclature has the indispensable advantage of being international. It is in fact a relic of the time when Latin was the universal language of learned men.

Genera, as has been said, are grouped into families, families into orders, orders into still larger groups, each successively higher grade of groupings containing forms which are less and less like those belonging to other groupings of the same grade.

Range of Form and Structure in the Plant World. - Starting with the higher forms, including the trees, shrubs and herbs with which we are most familiar, the following are the larger groups of plants :

(I) The Seed Plants (Spermophytes), including (i) the Angiosperms, or true Flowering Plants, which produce seeds completely enclosed in bag- or box-like structures, and comprise nearly all the herbs, grasses, etc., and the shrubs and broad-leaved trees, and (ii) the Gymnosperms, with seeds not so enclosed, but 
often borne in cones, as in the Coniferæ, mostly needleleaved trees (pines, firs, etc.). The Seed Plants have the most complicated internal structure of any plants.

(2) The Pteridophytes, plants of the same grade of organisation as the Ferns, and possessing, like the Seed Plants, stems, roots and leaves, and a well-developed internal water-conducting system, but reproducing themselves not by seeds, but by very minute bodies called spores. These include the Ferns, Clubmosses, and Horsetails. The living forms of the two lastnamed groups represent a very small proportion of the plants belonging to these groups, which flourished in certain past ages, and which, often growing to the size of trees, are preserved as fossils in the coal measures and other rocks. In the same rocks are preserved forms which are intermediate between the Pteridophytes and the Seed Plants. These formed seeds, but seeds which were different in structure from the seeds of existing plants.

(3) The BRYophytes, including the Mosses and the Liverworts, also reproducing themselves by spores, but much smaller on the whole than the Pteridophytes, and with a less well-developed water-conducting system, or none at all. The Mosses and some of the Liverworts have distinct stems and leaves, but other liverworts have none, the plant body consisting of a flat thallus, usually a branched ribbon-shaped structure, rather like an indefinitely growing leaf. These forms are mostly only able to live in relatively damp places, though the majority are land plants.

(4) The ALGex, plants living mostly in water, and able, like the previous groups, to form organic substances from inorganic. They all contain chlorophyll. but some of them, the red and brown seaweeds, for 
instance, are not green in colour, because the colour of the chlorophyll is modified by the presence of other pigments. They include the seaweeds, many of which are large plants, with a considerable degree of internal organisation, and the algæ that live in freshwater, most of which are pure green in colour, and consist of simple filaments or single microscopic cells (see below).

(5) The FungI, plants of about the same grade of organisation as the algæ, and, like them, comprising a great range of size and complexity of organisation, from the bulky mushrooms and toadstools to simple moulds and mildews and minute microscopic forms. They are distinguished by not possessing chlorophyll, and they depend on organic food.

(6) The Lichens, peculiar compound plants, each consisting of an alga and a fungus associated together in one plant body, or thallus, which resembles in a general way the liverwort thallus referred to above, though it often branches in a complicated way. The thallus of lichens is variously coloured: sometimes the green colour of the included alga can be seen through the fungal investment. Lichens are especially found on rocks, the trunks of trees, or the bare soil.

Many of the algæ and fungi are microscopic, and some of them are very minute indeed. Among these microscopic forms, which live in water or some other liquid, we come to the groups of organisms referred to on pp. 23-4, which are on the borderline between plants and animals, and are sometimes classed together as

(7) The Protista. Among these are the Bacteria, the smallest of all visible organisms, which are on the whole plants, but many have the animal character of locomotion. They are of enormous importance to mankind, as we shall see in a later chapter. The 
Protista include many other groups of simple organisms of very various characters.

As we descend the series outlined above, starting with the higher (most complex) plants, we find that the forms of plant life (with the exception of the fungi and lichens) are more and more dependent on external water, most of the algæ living all their life inmersed in water. It is established biological doctrine that the higher plants, which are able to live on dry land, though their roots must in fact always be able to obtain some water from the soil, have gradually arisen, during the history of the world, from forms like these lower plants which are confined to water. This is the doctrine of organic evolution, as applied to plants, a doctrine of whose truth Darwin first succeeded in convincing the world.

Cellular Structure of Plants.--When we come to examine their structure with the microscope, we find that the bodies of nearly all plants and animals are composed of what are called cells and the products of cells. In the case of plants the cells are closed spaces, surrounded by a cell wall of non-living substance, and containing living substance called protoplasm, in which all the ultimate life processes occur. This protoplasm is the only part of the bodies of living beings which is actually alive. A large part of the body consists of non-living organic substances formed by the protoplasm. Thus in plants the body is composed of a "skeleton" of cell walls, with living substance in each cell cavity. The protoplasm is, however, generally continuous from cell to cell by means of exceedingly thin filaments of protoplasm (which cannot be seen by ordinary microscopic observation) passing 
through the cell walls, so that all, or nearly all, the protoplasm of the plant really forms a single connected structure.

The protoplasm of many of the cells of the bodies of the higher plants disappears during the life of the plant, so that only the dead cell walls remain, for instance in the outer bark and the heartwood of tree trunks, which are thus dead, though integral parts of the living plant body, just as are the nails, for instance, in the case of man.

Green Colour of Plants. Chlorophyll. - The great majority of plants, from the simplest algæ to the flowering plants, are green, or have green parts (fungi and bacteria are an exception). It has already been mentioned that this green colour is due to the presence of a pigment called chlorophyll, whose presence, owing to the light which it absorbs, enables the protoplasm of the plant cell containing it to build up organic substances out of simple inorganic substances which the cell absorbs. The chlorophyll is contained in definite protoplasmic bodies called chloroplasts, usually spherical, oval or disc shaped, within the general protoplasm of the cell. This process of the formation of organic substances from inorganic by the activity of the chloroplasts under the action of light is called photosynthesis. I It is upon photosynthesis that the continuance of life in the world ultimately depends, because it is the only natural process which makes new organic substance on a sufficiently large scale.

Protoplasm, as has been said, is the seat of all the essential life processes. Before we can begin to analyse the nature and relations of these it is essential to learn something of the nature of protopiasm itself. Though

x "A putting together with the help of light," from Greek $\phi \tilde{\omega}$,

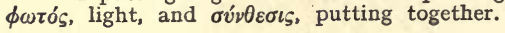


we are far from understanding the whole secret of life, we do know that many of the manifestations of life depend upon the chemical nature and physical properties of protoplasm and of the substances derived from it. The next two chapters will therefore be devoted to these subjects.

\section{PRACTICAL WORK.}

\section{A. Use of the Microscope.}

(I) Examine the microscope, especially the mirror, substage diaphragm, low and high power objectives, eyepieces, coarse and fine focussing adjustments. [The demonstrator should show the students how to handle a microscope, explain the outlines of its working, and give the necessary cautions.]

(2) Put a small drop of water on the middle of a slide, and place in it a few grains of sand. Cover with a coverslip so as to include in the water some bubbles of air.

Examine first with the low power and then with the high power

(a) a sand grain, noting the shape, colour and translucency ;

(b) an air bubble, noting its appearance in different focal planes.

(3) Mount a single leaf from a moss plant in a drop of water. Cover with a coverslip so as not to include bubbles of air.

Examine first with the low and then with the high power. Note that the leaf consists of a single layer of box-like compartments (cells), with transparent walls, through which can be seen the green chloroplasts containing the pigment chlorophyll. The cells of the moss leaf are a good type of green plant cell.

(4) Determine the apparent length in $\mu^{x}$ in the field of the microscope (with fixed tube length) of a division of the eyepiece micrometer scale, $(a)$ with the high and $(b)$ with the low power objective, by determining the correspondence of the eyepiece scale with the divisions of the measured scale engraved on the micrometer slide. Make a note of these values for future use. Now measure the length and breadth of a cell of the moss leaf and the diameter of a chloroplast under the high power.

$$
{ }^{2} \mu=\text {.oor mm. }
$$




\section{B. Types of the Plant Kingdom.}

A series of specimens will be given out by the demonstrators to be handed round. These illustrate types of all the great groups of the plant kingdom, from the highest (flowering) plants. Ask the demonstrator any questions that occur to you about them.

Examine also the fully labelled demonstration series.

\section{Differences between Species of the Same Genus.}

Examine carefully fresh specimens of two or three species of flowering plant belonging to the same genus. Write down the differences which strike you, and get the demonstrator to correct your notes and to point out the characteristic differences in case you have failed to observe them. [The species used must depend upon the time of year and upon what can be obtained. In the autumn $Q$ uercus robur, $Q$. sessiliflora and $Q$. ilex are suitable; in April Veronica agrestis, $V$. hederifolia and $V$. tournefortii; in May or June Ranunculus acer, R. repens and R. bulbosus.]

\section{Exceptions to the Characteristic Forms and Structures of Plants and Animals.}

\section{(Demonstration Specimens.)}

(I) A Branching Animal.-Fresh or museum specimens of a hydroid polyp or a bryozoon " colony." Each individual has a mouth surrounded by tentacles and a gut. It consumes solid food consisting of tiny organisms living in the sea, and is therefore an animal in spite of the facts that it is fixed to one spot and that the whole " colony" has a branching form like a plant.

(2) A Compact Plant.-Mamillaria, or other cactus from subtropical America, shows no obvious division of its shoot into stem and leaves. It has a branching root in the soil like an ordinary plant, but its shoot consists of a compact fleshy green stem bearing spines or bristles which represent the leaves. The compact form decreases the evaporating surface and at the same time stores water which is held by the abundant mucilage the plant contains, so that the cactus is able to live in a hot, dry climate.

(3) An Animal with no Mouth.-The tapeworm is a parasitic animal which absorbs its liquid organic food from the intestines of the animal in which it lives. It has no mouth or gut, having lost these by degeneration in correspondence with its habit of life. 
(4) A Plant which consumes Solid Organic Food.-The sundew (Drosera) and the pitcher plant (Nepenthes) are insectivorous plants which digest and absorb the products of insects that fall on to the leaf or into the pitcher (a modified part of the leaf). This is a character not possessed by most plants, but it serves to demonstrate the common powers of plant and animal protoplasm. In other respects the sundew and the pitcher plant are ordinary flowering plants. 


\section{ORGANIC SUBSTANCES AND THEIR CHEMICAL CHARACTERS}

AlL the activities of life depend upon protoplasm, which is therefore the essential part of every living organism. But by no means all parts of an organism consist of or contain living protoplasm. For instance, the heartwood and outer bark of a tree, the hairs, feathers, nails or hoofs of a warm-blooded animal are destitute of protoplasm. These lifeless parts of an organism are, however, all formed from or by protoplasm, and they consist mainly of complex chemical compounds, containing carbon, hydrogen and oxygen, often also nitrogen and sulphur, as well as other elements, which are called "organic" compounds because they are associated with organisms. There is no sharp distinction between organic and inorganic compounds in chemistry, and many of the simpler organic compounds formed by organisms can also be made synthetically in the laboratory.

All the organic compounds contain the element carbon, organic chemistry being sometimes known as "the chemistry of the carbon compounds," though many substances containing carbon (for instance, calcium carbonate) are not specially associated with organisms. Of the immense number of organic compounds known certain classes are specially important in the structure and actıvities of living organisms, and 
we must have some knowledge of the nature and properties of these before we can understand the nature and functions of protoplasm.

We must, of course, be careful to distinguish between an organic substance which is a mixture of chemical compounds in various proportions, and a chemical compound which has a perfectly definite chemical composition, i.e. its molecule or ultimate unit of structure consists of a definite number of atoms of different elements arranged in a definite way. Milk, for instance, is an organic substance which has a varying composition, being a mixture in various proportions of the chemical compounds water, milk sugar, various definite fats, proteins, salts, etc. Such organic substances as wood, horn, hair, etc., are, similarly, mixtures of chemical compounds.

Of the chemical compounds which enter into the composition of the body of an organism, water $\left(\mathrm{H}_{2} \mathrm{O}\right)$ is of first importance, as we shall see in detail later. It is essential to the structure of living protoplasm, and is found in greater or less proportion in all parts of the body. More than 90 per cent. of the weight of a herbaceous plant is water, as can easily be shown by weighing the plant and then heating it at $100^{\circ} \mathrm{C}$. till it loses no more water and weighing again.

The three classes of chemical compounds which play the leading part in organisms are the carbohydrates, the fats, and the proteins, the two former consisting of carbon, hydrogen and oxygen, the latter with nitrogen, sulphur, and sometimes phosphorus in addition.

\section{The CaRbohydrates.}

These are a class of carbon compounds in whose molecules the atoms of hydrogen and oxygen are 
generally present in the proportion of two to one, as in water.

\section{The Sugars.}

The most important carbohydrates are the sugars, of which there are many different kinds. Glucose (grape sugar), $\mathrm{C}_{6} \mathrm{H}_{12} \mathrm{O}_{6}$, lavulose, $\mathrm{C}_{6} \mathrm{H}_{12} \mathrm{O}_{6}$, sucrose (cane sugar), $\mathrm{C}_{12} \mathrm{H}_{22} \mathrm{O}_{11}$, and maltose, $\mathrm{C}_{12} \mathrm{H}_{22} \mathrm{O}_{11}$, are the only ones we need mention here. It will be seen that the two former have the same number of atoms in the molecule, and so have the two latter. They differ, however, in the way in which the atoms are grouped, and this leads to differences in crystalline form, and to differences in reaction with other compounds.

The sugars are readily soluble in water; moreover, they easily move about the body by liquid diffusion, and their molecules possess a large amount of potential (chemical) energy, so that when the molecule is oxidised and broken up a great deal of energy is set free in the kinetic form. The kinetic energy which appears in organisms as heat and movement is mainly derived from the oxidation and breaking up of sugars. For these reasons the sugars are extremely important in the sum total of the chemical changes, called metabolism, which are perpetually occurring in the living protoplasm of plants and animals.

Glucose (grape sugar), $\mathrm{C}_{6} \mathrm{H}_{12} \mathrm{O}_{6}$, is the most prominent of the sugars in the economy of organisms. While glucose is a relatively stable substance in the test tube, it takes up oxygen more easily than other sugars, and thus acts as a moderately strong "reducing agent," i.e. it can take oxygen from (or " reduce ") certain other substances. An example of this action is the behaviour of glucose solution when mixed with a cupric salt (i.e. a salt of copper containing much oxygen) in alkaline 
solution-such as Fehling's solution-and warmed. Some of the oxygen is taken from the cupric salt, and red cuprous oxide is formed. This is the ordinary test for sugars that behave in this way-the "reducing sugars" as they are called. Maltose is also a reducing sugar, though to a less extent than glucose. On the other hand, sucrose (cane sugar) does not reduce Fehling's solution at all.

In the living cell glucose very readily takes up oxygen and breaks up its molecule into carbon dioxide and water. This is the basis of the process of respiration, which is of fundamental importance in the economy of living organisms:-

$$
\underset{\text { glucose }}{\mathrm{C}_{6} \mathrm{H}_{12} \mathrm{O}_{6}}+\underset{\text { oxygen }}{6 \mathrm{O}_{2}}=\underset{\substack{\text { carbon } \\ \text { dicxide }}}{6 \mathrm{CO}_{2}}+\underset{\text { water }}{6 \mathrm{H}_{2} \mathrm{O}}
$$

Sucrose or saccharose (cane sugar), $\mathrm{C}_{12} \mathrm{H}_{22} \mathrm{O}_{11}$, is the most important sugar commercially. It is the main product of the sugar cane and the sugar beet, and is the principal sugar we use as food. It is very much sweeter than glucose. The importance of sugar as a food for animals depends on the characters already mentioned, solubility and high potential energy. It is thus easily absorbed through the wall of the alimentary canal into the blood, and the readiness with which it is oxidised places large amounts of energy at the disposal of the muscles, where the energy appears in the kinetic form, as motion and heat. Sugar is, in fact, used in this way by all living cells, but most energetically by the muscles, which are the great energy spenders of the animal body.

Sucrose is not a reducing sugar, and, in the living cell, is converted into glucose and lævulose before being oxidised. This process of the conversion of sucrose 
into glucose and lævulose is an example of hydrolysis, i.e. the splitting of a molecule by reaction with water.

$$
\underset{\text { sucrose }}{\mathrm{C}_{12} \mathrm{H}_{22} \mathrm{O}_{11}}+\underset{\text { water }}{\mathrm{H}_{2} \mathrm{O}}=\underset{\text { glucose }}{\mathrm{C}_{6} \mathrm{H}_{12} \mathrm{O}_{6}}+\underset{\text { lævulose }}{\mathrm{C}_{6} \mathrm{H}_{12} \mathrm{O}_{6}}
$$

Various sugars are found mixed in different proportions in living cells and are constantly being converted from one form into another.

\section{The Polysaccharides.}

These are another important class of carbohydrates. They derive their name from the fact that they are formed by the putting together of many molecules of sugar to form one molecule of polysaccharide, a process known as condensation. The most important polysaccharides of plants are the starches and the celluloses. There are many different kinds of each, but for our purpose we may treat them in each càse as one.

Starch is formed as solid grains in the protoplasm of the living cells of plants. The substance of each grain is laid down in concentric layers round a centre called the hilum of the starch grain. Starch is formed by the union of many molecules of glucose with the elimination of one molecule of water from each :-

$$
n \mathrm{C}_{6} \mathrm{H}_{12} \mathrm{O}_{6}=\left(\mathrm{C}_{6} \mathrm{H}_{10} \mathrm{O}_{5}\right)_{n}+n \mathrm{H}_{2} \mathrm{O}
$$

The value of $n$ has not been exactly determined, but it is probably somewhere about roo. Thus the starch molecule is far more complex than the glucose molecule. Starch has a very characteristic blue reaction with watery solution of iodine in potassium iodide. Most starches are quite insoluble in cold water ; they are hydrolysed in the presence of dilute mineral acids into various simpler carbohydrates, and eventually 
into glucose. Hydrolysis of starch also takes place in plant cells (see below). Starch is a very common and widespread substance in plant cells. It bulks largely in the economy of plants, though it stands second to the sugars in this respect. It also forms a very valuable food for animals, being hydrolysed into sugars (or rather its hydrolysis is catalysed) by the action of the digestive juices of the mouth and of the small intestine.

Cellulose is a substance of even more complex composition than starch. Its empirical formula is the same, i.e. it has the same proportion of carbon, hydrogen and oxygen atoms in the molecule, though a much higher total number. Thus, if starch be represented by $\left(\mathrm{C}_{6} \mathrm{H}_{10} \mathrm{O}_{5}\right)_{n}$ we may represent cellulose by $\left(\mathrm{C}_{6} \mathrm{H}_{10} \mathrm{O}_{5}\right)_{m}$.

Cellulose is of immense importance because it forms the main substance of the cell walls of most plants, and thus the "skeleton" of the plant body. Linen and cotton are nearly pure cellulose, and paper contains a varying amount according to the plant substance from which it is made. Cellulose is formed in a similar manner to starch by the condensation of many molecules of sugar. It is not coloured blue with iodine, but it can be altered, e.g. by the action of strong sulphuric acid into a substance which resembles starch in so far as it is so coloured with iodine.

\section{The FATS.}

These, like the carbohydrates, consist of carbon, hydrogen and oxygen, but the fat molecule is much larger than the sugar molecule, and contains a much smaller proportion of oxygen. Fats are widely distributed in plant cells, and form a characteristic store of non-nitrogenous food in many seeds, e.g. linseed, 
cottonseed, coconut, castor oil seed. They contain an even larger store of potential energy per molecule than the carbohydrates, and thus require more oxygen for complete oxidation. Of numerous kinds two examples are palmitin $\left(\mathrm{C}_{51} \mathrm{H}_{98} \mathrm{O}_{6}\right)$ and olein $\left(\mathrm{C}_{57} \mathrm{H}_{104} \mathrm{O}_{6}\right)$. Fats occur in plant cells as complex mixtures of different individual fats. They are usually found in the liquid state, and are then often spoken of as " oils."

Fats are even more widespread and important in the animal than in the plant body, forming a very valuable reserve store of energy.

\section{The Proteins.}

These are the most important of the nitrogencontaining organic substances. The molecule consists of carbon, hydrogen, oxygen, nitrogen and sulphur, with sometimes phosphorus in addition. The protein molecules are the largest and most complex of any chemical compounds, each molecule consisting of many hundreds, or even thousands, of atoms. The different groups of atoms composing these huge molecules have, like the atoms composing the molecules of all chemical compounds, a perfectly definite position in regard to one another. The more numerous the atoms the greater will be the number of different relative positions possible. Hence there are an enormous number of distinct protein molecules with comparatively small differences between them.

Now protein molecules form the essential basis of protoplasm. Protoplasm, as we shall see in succeeding chapters, has the same general characteristics and powers in all living organisms, but it is obvious that the protoplasm of every species differs in some way from that of every other species, or it 
could not build up a different body. It is probable that differences between species ultimately depend upon comparatively slight differences in structure between some of the proteins present, or between the relative amounts of the different proteins present, or perhaps between the ways in which different protein molecules are built up into more complex protoplasmic structures, of which we actually know practically nothing. Such biochemical differences between even closely allied species have recently been shown to hold for other important organic substances of the body, for instance between the starches of different species of plants and between the hæmoglobins (the red pigment of the blood) of different species of animals. It has been shown that the starch of every species (out of many hundreds examined) is in some respect different from the starch of every other species; and the same is true of hæmoglobin. The proteins are much more difficult to investigate, but when we consider that the molecules of many of them are much more complicated, it becomes obvious that the possibilities of slight but perfectly definite differences are much more numerous.

Proteins as a class show certain colour changes when acted upon by certain substances; for instance a yellow colour, when after boiling with nitric acid ammonia is added; a violet colour, with a drop of dilute copper sulphate followed by excess of caustic soda. A dilute solution of iodine in potassium iodide, which colours starch blue, stains proteins yellow. In such ways proteins, as a class, can be distinguished from other classes of organic compounds. Into the characters of the different proteins we shall not attempt to enter here.

Apart from their universal occurrence in living proto- 
plasm, as the essential part of its structure, proteins occur in more or less solid aggregations as grains (called "aleurone grains") of non-living substance in the cells of certain parts of plants, such as seeds. Here they represent a store of protein substance used as food by the young seedling, and are comparable in this respect with starch grains or oil (fat) globules, which similarly form stores of non-nitrogenous substance.

There are of course many other classes of organic substances besides the three mentioned. In plants, for instance, the so-called "aromatic substances" occur universally. They contain carbon, hydrogen and oxygen, and are founded on the structure known as the benzene ring, or a similar ring, but our knowledge of their rôle in the economy of plants is still obscure. The three classes of organic substances-the carbohydrates, fats and proteins-briefly described comprise the most inportant organic substances entering into the composition of organisms, and they can all be used as foods by animals.

Enzymes.-These are a very important class of substances, whose exact chemical composition is still unknown, but many of them can easily be isolated. Their importance consists in the fact that they act as catalysts to many of the chemical changes occurring in living protoplasm. A catalyst is a substance that facilitates a chemical change without itself entering into the final products of the change. It probably works by temporarily entering into combination with the substance or substances in reaction, causing a disruption of their molecules, and ultimately separating from the products. Owing to the fact that the enzyme itself is not involved in the final product, a very small 
amount of enzyme is sufficient to carry out the change in large quantities of substance.

Enzymes in great variety exist in the living body, each kind acting upon a particular class of substance. Thus the lipases I facilitate the hydrolysis of fats, which are decomposed into simpler substances; the proteases have a similar action upon proteins. Of the enzymes which work upon carbohydrates, diastase, which converts starch into sugar, is the best known. The first stage of the reaction produces dextrin (a soluble polysaccharide of the same empirical formula as starch) and maltose :-

$$
\underset{\text { starch }}{\left(\mathrm{C}_{6} \mathrm{H}_{10} \mathrm{O}_{5}\right)_{n}}+\underset{\text { water }}{\mathrm{H}_{2} \mathrm{O}} \rightarrow \underset{\text { dextrin }}{\left(\mathrm{C}_{6} \mathrm{H}_{10} \mathrm{O}_{5}\right)_{x}}+\underset{\text { maltose }}{\mathrm{C}_{12} \mathrm{H}_{22} \mathrm{O}_{11}}
$$

The molecule of maltose is hydrolysed (its hydrolysis being catalysed by another enzyme, maltase) into two molecules of glucose :-

$$
\underset{\text { maltose }}{\mathrm{C}_{12} \mathrm{H}_{22} \mathrm{O}_{11}}+\underset{\text { water }}{\mathrm{H}_{2} \mathrm{O}}=\underset{\text { glucose }}{2 \mathrm{C}_{6} \mathrm{H}_{12} \mathrm{O}_{6}}
$$

Invertase similarly catalyses the hydrolysis of sucrose into one molecule of glucose and one molecule of the closely allied sugar lavulose:-

$$
\underset{\text { sucrose }}{\mathrm{C}_{12} \mathrm{H}_{22} \mathrm{O}_{11}}+\underset{\text { water }}{\mathrm{H}_{2} \mathrm{O}}=\underset{\text { glucose }}{\mathrm{C}_{6} \mathrm{H}_{12} \mathrm{O}_{6}}+\underset{\text { lævulose }}{\mathrm{C}_{6} \mathrm{H}_{12} \mathrm{O}_{6}}
$$

Cytases facilitate the hydrolysis of the celluloses into simpler soluble carbohydrates.

- Many, if not all, of the enzymes have what is called a reversible action, i.e. they may bring about a change in either direction, e.g. of starch into sugar, or of sugar into starch. The direction in which the reaction actually proceeds at any given moment depends upon the relas The uniform termination -ase is used in the naming of enzymes. 
tive concentration of the soluble end products. Thus, if the sugar concentration is low, starch will be converted into sugar, but when it rises above a certain point the sugar will be converted into starch. We shall see in the sequel that these reversible reactions are very important in the living organism.

In nature organic compounds always occur as constituents or products of living organisms, but chemists can now make many of them out of simpler substances in the laboratory, for instance the sugars, and even some of the simpler proteins.

\section{PRACTICAL WORK.}

\section{Carbohydrates, Fats and Proteins.}

(I) Compare the samples of solid glucose and of solid sucrose provided. Note that the first is a crystalline powder, while the second forms large well-defined crystals. Compare their sweetness by tasting. Dissolve the samples in two separate test tubes each containing a little water, and note that each dissolves completely. Test a little of each with very dilute solution of iodine in potassium iodide $x$ and note that there is no coloration. Add a few drops of Fehling's solution (copper sulphate made strongly alkaline) to each test tube, and heat. Note that one solution turns red owing to the formation of red cuprous oxide, while the other does not.

(2) Examine a thin slice of potato under the low and high powers of the microscope. Draw one or two of the starch grains under the high power. Measure their long and short diameters with the micrometer eyepiece and express the values in $\mu$ (see Practical Work I (4) p. 33). Treat the section with very dilute iodine solution and examine again.

(3) The tube provided contains very dilute starch paste. Pour a little into a watchglass, and add one drop of very dilute iodine solution. Now add a little taka-diastase (a commercial preparation of diastase also containing maltase, see p. 45) to the contents of the tube, and shake the tube vigorously at intervals. After a minute pour a little of the mixture into another watch-

I This solution will be alluded to in future simply as "iodine solution." 
glass and add a drop of very dilute iodine. Shake the tube vigorously again at intervals and after another two minutes pour a little into a third watchglass, adding a drop of very dilute iodine. Note the colours in the three cases. The pinkish colour with iodine is due to the formation of dextrin, a carbohydrate intermediate between starch and sugar. On adding a few drops of Fehling's solution to the contents of the test tube after half an hour, and heating, a slight reducing effect should be obtained. This is due to the glucose present.

(4) Test with iodine solution a piece of good writing paper (nearly pure cellulose) across which strong sulphuric acid has been streaked.

(5) Examine the demonstration specimen of paper boiled in strong sulphuric acid, neutralized with alkali and then treated with Fehling's solution to demonstrate the formation of a reducing sugar (glucose); also of the cut date stones (the very thick walls of whose cells consist of cellulose) similarly treated.

(6) Note that desiccated coconut "meat" (food material used by the embryo growing into a seedling) is oily to the touch. Place some in a test tube with water and boil: the oil rises to the surface. Add a few drops of Sudan 3 (an aniline dye) and allow to stand: the oil is coloured red. Add a few drops of Sudan 3 to olive oil in a test tube : the oil is coloured red. Pour half the red oil into another test tube. Add a little alcohol to one and a little water to the other. Note the position taken up by the water and alcohol respectively in relation to the oil: also the coloration resulting.

(7) The two substances provided in separate test tubes are (a) white of egg shaken up with water, which is a typical protein, (b) bean meal shaken up with salt solution. Divide each into three portions and subject each set of three to the following tests, noting the coloration in each case:-

(i) Add a few drops of strong nitric acid. Boil and then cool thoroughly. Add a few drops of ammonia solution) xanthoproteic reaction).

(ii) Add one drop of dilute copper sulphate solution. Now add excess of caustic soda or potash (biuret reaction).

(iii) Add a few drops of iodine solution. 


\section{SOME PHYSICAL CHARACTERS OF ORGANIC SUBSTANCES}

Crystalloids and Colloids.-A solid substance, when placed in water, may behave in one of several ways. First, it may react chemically with the water, as for instance sodium, which breaks up the molecule of water, combining with the oxygen and liberating hydrogen. Secondly, it may dissolve in the water to form a true solution, as for instance common salt (sodium chloride), the salt seeming to disappear in the solution (where it is called the solute, the dissolving substance being the solvent). Thirdly, it may remain practically unaltered, as for instance quartz sand (silica). If, however, such a substance as the coarse grains of silica are ground in a very finely grinding mill into finer and finer particles, a point of fineness is ultimately reached at which the particles stay suspended in the water, without settling, to form what is known as a colloid I solution, or sol, which differs from a true solution in several important respects. This fourth kind of behaviour with water is a very characteristic feature of many organic compounds.

Broadly speaking, the substances which form true solutions with water, or some other liquid solvent, have small or comparatively small molecules, such for instance as simple inorganic salts, and many of the ${ }^{x}$ Greek $\kappa \dot{\alpha} \lambda \lambda a$, glue, from the jelly-like nature of the most familiar colloids ( $\mathrm{gels}$ ). See p. 53 . 
simpler organic compounds, such as the sugars. On evaporation of the solvent they reappear, often in the form of crystals. Substances which behave in this way are known as crystalloids. The substances which when mixed with water become dispersed in very fine particles through the water, each particle consisting of a number of molecules aggregated together, are called colloids. It is to be noted that many simple substances - even elements such as gold and platinum-may be prepared in such a form that they behave as colloids with water, i.e. they become dispersed in minute particles through the liquid. We now know that we cannot sharply divide substances into the two categories, crystalloids and colloids, but speak of a substance, in a given condition, behaving as " a crystalloid or as a colloid" with water, or some other solvent. Nevertheless the organic compounds with large molecules, e.g. polysaccharides, proteins, etc., commonly do behave as, and are therefore commonly spoken of as, colloids.

The aggregate of the dispersed particles of a colloid is known as the disperse phase, the continuous liquid medium in which they are dispersed as the continuous phase of the colloid sol. The disperse phase does not necessarily consist of solid particles, it may consist of very minute liquid droplets, and then the sol is called an emulsion sol, or an emulsoid, while if the disperse phase is solid we have a suspension sol, or a suspensoid.

Coarse particles or droplets, suspended in a liquid, form ordinary suspensions or emulsions, and tend to sink slowly to the bottom, or rise to the top, as they are heavier or lighter than the liquid. Thus the fat globules suspended in milk slowly rise to the surface as cream. If we shake up a handful of soil in a beaker of water, the coarser and heavier particles sink to the 
bottom at once, others sink more slowly, but the very fine colloidal particles (clay) remain suspended indefinitely, forming a colloid sol with the water.

\section{Microscopic and Ultramicroscopic Particles.-The} larger of these very fine colloid particles, such as those of an Indian ink sol, may be clearly seen under the high power of the microscope. When they are quite freely suspended, that is not adhering to the glass slide or coverslip, they may be seen to be in continuous oscillation, or intermittent jerky movement. This is due to the continuous bombardment of the disperse particles by the molecules of the liquid, which are of course themselves in constant motion. At any given moment the probability is that this bombardment will be unequal on different sides of the suspended particle, and this will therefore move away from the side on which it is most heavily bombarded. If the bombardment happens to be momentarily equal on all sides the particle will remain motionless. This kind of movement is called Brownian movement, from its discoverer, the botanist Robert Brown.

The disperse particles of the finer sols, such for instance as a Congo Red sol, are too small to be seen under the highest powers of the microscope, and the sol appears clear. With the highest powers and the best definition the smallest particles that can be clearly defined under the microscope are those not less than about ${ }^{\prime} 5 \mu^{\mathrm{I}}=$.000I5 mm. in diameter. But by means of an instrument called the ultramicroscopewhich consists essentially of a high power microscope with lateral instead of vertical illumination-when lighting from the side and viewing against a dark

${ }^{1} \mu$ is one-thousandth part of a millimeter and is the ordinary unit of microscopic measurement. 
background, smaller objects than these can be detected, though they cannot be clearly defined. They are seen as tiny blurs of light owing to diffraction of the rays round the ultramicroscopic particles. By this method particles of about $005 \mu(=5 \mu \mu)$ I may be detected, which we know from calculations based on other data is about the size of the very large molecules of soluble starch. ${ }^{2}$ The disperse particles of very fine sols run down to perhaps $2.5 \mu \mu$, the size of the molecules of hæmoglobin, the red pigment of the blood. The molecules and ions (single atoms, or small groups of atoms, into which a fine solute may be dissociated) of true (crystalloid) solutions are much smaller than this, from $5 \mu \mu$ (alcohol molecule) down to perhaps ' $I \mu \mu$, which is about the diameter of a hydrogen ion (separate atom of hydrogen).

To give some more easily comprehensible notion of the relative sizes of these exceedingly minute objects : if we imagine the hydrogen ion magnified a million times, so that it was just visible to the naked eye as the smallest possible ink dot on a sheet of paper, then a very large organic molecule (such as might just be detected with the ultramicroscope), if it were magnified to the same extent, would be about the size of a small pea, and a very small bacterium (the smallest organisms that are just visible with the microscope) would be twice the diameter of a football. Represented on this scale a real pea would appear more than three miles in diameter.

Internal Surface of Colloids.-The very large number of particles or droplets forming the disperse phase of

\footnotetext{
' $\mu \mu$ is one-thousandth of $I \mu$, and is the unit of ultramicroscopic measurement.

2 Soluble starch is a simpler form of starch produced on boiling with dilute hydrochloric acid and forming a colloid sol with water.
} 
a colloid soil present collectively an enormous surface. If we take a solid spherical object, such as an orange, and cut it into halves, it is clear that we have greatly increased the exposed surface, for to the original external surface we have added the cut surfaces of the two halves. If we now cut the halves into quarters the total surface is still further increased, and the more we divide the orange the greater the surface exposed. Thus the collective surfaces of all the disperse particles in a drop of a colloid sol are immensely greater than the surface of the sphere which would be formed if all the particles in the drop were aggregated in a single mass.

\section{Surface Energy, Surface Tension and Adsorption.-} Now, where a surface exists, i.e. where solid or liquid matter is in contact with other matter, that surface is the seat of free energy, because of the break in action of molecular forces at the surface, and this is expressed in what is called surface tension. The surface of a drop of liquid in air, for instance, tends to contract, and that is why a free drop of liquid assumes the spherical shape, in which the surface has contracted as much as possible, and has the smallest possible area in relation to the mass of the liquid. Liquids with a high surface tension form drops which do not easily spread upon a solid surface, because their high surface tension tends to keep the drop spherical and prevent it spreading. Mercury, water and alcohol form a series of liquids with decreasing surface tension, and a corresponding increasing readiness to " wet," i.e. spread upon, a clean sheet of glass.

The enormous collective surface, i.e. the sum of all the surfaces, of the disperse particles or droplets of a sol involves a corresponding amount of surface energy 
of the particles or droplets. This is of very great importance in determining the behaviour of the colloid towards liquids, solutes and other sols, which may be attracted to the surfaces of the disperse particles or droplets, and held there by the surface energy, at the same time decreasing the surface tension of the disperse phase. This process is called adsorption. The living protoplasm of the cell is believed to be a colloid sol of which the continuous phase is a solution of various crystalloids, and the disperse phase consists of protein and fat droplets or particles. These take up and hold by adsorption the molecules and ions of solutes which can diffuse into the cell and come within the range of their surface energy. In this way large quantities of various substances may be taken in and held in the living cell. Dyes are taken up in the same way by protoplasm and by the other organic colloids of the plant or animal, and on this process largely depends the staining of tissues employed in making microscopic preparations.

Gel Formation.-When hydrosols (sols with water as the continuous phase) lose water, for instance by evaporation, their disperse particles get closer and closer together. In the case of certain sols, including those whose disperse phases consist of large organic molecules, such as those of gelatine for example, the molecules or aggregates of molecules tend, on loss of water, to run together in chains. As more water is lost, we picture these chains becoming more and more entangled with one another, the sol becomes more and more viscous (treacle-like), till ultimately a jellytechnically called a gel-is formed. On further loss of water the gel shrinks, and eventually becomes solid. If water is again added it is absorbed, and the substance 
gradually swells till it passes back into the condition of a sol.

A gel which can thus pass back into a sol is called a reversible gel. A piece of solid gelatine, for instance, will absorb water till it passes into the gel, and finally into the sol condition, in which the original gelatine has disappeared from sight. Allowed to evaporate the sol will lose water till the gel reappears and the gelatine finally becomes solid again; and these opposite processes can be carried out an indefinite number of times.

Many organic colloids of the living body are in the gel condition, e.g. cell walls, starch grains, gums, mucilages, etc., and some of these, by the absorption and loss of water, can pass into sols and back again. Protoplasm itself may exist either as a sol or as a gel. The internal surface of a gel, though less than that of the disperse phase of a sol, is nevertheless very great, and gels accordingly show those characters, such as the power of adsorption, which depends upon internal surface.

Membrane Formation.-Gels may be formed from sols in other ways than by loss of water. For instance, when a sol is in contact with some other substance, a film or membrane of gel structure is often formed on the plane of contact. The protoplasmic sol always forms a menibrane or film on its free surface, and these protoplasmic gel membranes play a vital part in the economy of the living cell.

Diffusion.-A very important difference between crystalloids and colloids, a difference used by Thomas Graham, who first investigated the subject in the middle of last century, to distinguish between them is their relative diffusibility. 
If a strong (concentrated) solution of a crystalloid is brought into contact with a weak (dilute) solution of the same crystalloid in the same solvent, diffusion proceeds until the solute is equally distributed through the whole of the liquid. This is an expression of the general physical law that all systems tend towards equilibrium. The rate of diffusion varies directly with the difference in concentration of the two solutions, and with the temperature, inversely with the size of the molecule of the solute. If now the two solutions of different concentrations are separated by a colloidal membrane of the nature of a gel, such as vegetable parchment, crystalloid solutes will diffuse through the membrane as they would diffuse into a liquid with which they were directly in contact, though less rapidly. The rate of this diffusion through a membrane depends partly on the nature of the membrane, and partly on the size of the molecule of the solute, partly again on the chemical relation between the solute and the membrane.

The disperse phase of a colloid sol, on the other hand, does not in general pass through a gel membrane at all, or only does so with extreme slowness, and this again depends on the size of the particles, on the nature of the membrane, and on the chemical relations between them. It was this difference which Graham used to distinguish colloids from crystalloids, and he showed that a colloid and a crystalloid in mixed solution could be separated by placing the solution in a parchment bag and plunging the bag into water, when the whole of the crystalloid would eventually escape through the membrane, leaving the colloid pure behind. This process is called dialysis.

We may say, broadly, that crystalloids in solution 
will dialyse, and that colloids will not. But different crystalloid solutes pass through a given membrane at very different rates. The membrane may be likened to a sieve with meshes of definite size, though varying within limits. These meshes will let through molecules up to that size, but not larger ones. The disperse particles of a colloid sol are in general too large to pass through the membrane, but in some cases they may be just small enough to pass in small numbers. The matter is further complicated, as we have seen, by the reaction in some cases between the molecules of the solute or disperse phase and those of the membrane.

A membrane which will allow a solute to pass through it is said to be permeable to the solute. But a membrane which is permeable to the solvent and not to the solute is said to be semi-permeable. Different colloidal membranes show very different degrees of permeability to different crystalloid solutes, some letting through a large variety of solutes, others being impermeable to many, while others are impermeable to practically all solutes, though they allow water to pass. This fact has a great importance in the living cell because the gel membrane or film on the surface of the layer of protoplasm which lines the cell wall is a semipermeable membrane, allowing certain solutes to pass quickly, others slowly, and others, again, not at all.

Osmosis.-If we place some sugar solution in a parchment bag (i.e. a colloidal membrane) open at the top, and immerse the bag in water up to the level of the sugar solution within the bag, we find that the level of the liquid within the bag gradually rises owing to the passage of water from the beaker through the membrane, while the sugar does not pass out into the beaker. The membrane is said to be "semi-permeable 
to sugar solution," the molecules of the solute being unable to pass through it, while the molecules of water can. If common salt solution, instead of sugar, is within the bag, the molecules of salt can pass out (though at first the liquid rises in the bag because the water passes in more quickly than the salt can pass out), since the membrane is permeable to them. The solution within the bag becomes less concentrated owing to the entrance of water and the escape of salt, while the liquid outside becomes a salt solution of increasing strength till the two are of equal concentration and equilibrium is attained. But in the case of the sugar equilibrium cannot be attained in this way because the sugar molecules cannot escape through the membrane, and so the water continuously enters, rising in the bag if it is open at the top, distending its extensible wall if the bag is closed. This process of the passage of a solvent through a semi-permeable membrane which will not allow the solute to pass is called osmosis, and the pressure developed on the wall of the membrane is known as osmotic pressure.

Various solutes, among which sugars are prominent, to which the protoplasmic membranes bounding the cells appear to be practically impermeable, are formed within the living cells of plants and animals. These are known as osmotic substances, and many of the processes of absorption and movement of water within the organism depend on the passage of water through membranes on the other side of which such solutes exist, in accordance with the universal tendency to establish equilibrium.

We now begin to see the importance of the fact that the structure of organisms is so largely built up of 
colloidal substances. On the one hand we have the power of the colloid gel to absorb water, and the power of both gels and sols to adsorb a great variety of substances as a result of their immense internal surfaces. On the other we have the fact that the gel membrane, especially the protoplasmic membrane, is semi-permeable, so that it does not allow of the escape of osmotic substances within the cell, while it does allow water to enter freely, and other substances, such as salts, at various rates.

The colloidal nature of complex organic substances largely depends on the great size of their molecules, and this is most conspicuously seen in the proteins which form the basis of protoplasm. The great size of these complex molecules is often associated with instability of particular arrangements of the atoms which compose it. This makes possible the existence of very numerous chemical compounds of distinct though closely similar molecular structure. A given group of atoms within the molecule is replaced by another of somewhat different arrangement. This is the cause of the extraordinary richness and variety of the chemical and physical changes within the cell which are the basis of the varied manifestations of life and of organic form.

Though we are still very far from understanding exactly how what we call living protoplasm actually carries out all the complex processes which take place within it, we can already trace the direct dependance of many of them on the chemical and physical structure and relations of the substances of which protoplasm is composed. 


\section{PRACTICAL WORK.}

\section{A. Behaviour of Crystalloids and Colloids with Water.}

(I) Place some crystals of potassium bichromate at the bottom of a test tube and just cover with hot water. The crystals quickly dissolve and disappear and there is no expansion on solution. Examine a drop of the solution under the microscopeit is clear, no particles are to be seen. This is a true (crystalloid) solution. Leave the tube of solution and examine later: as the solution cools and the solvent evaporates, crystals of the salt reappear.

(2) Warm some powdered glue in a test tube with a little water: the glue disappears gradually, taking up water and forming a colloid sol. On cooling and standing a solid jelly ( $\mathrm{gel}$ ) is formed.

(3) Place a rectangular strip of dry gelatine on a sheet of glass over squared paper. Mark the edges of the gelatine with Indian Ink opposite the divisions of the paper. Allow the ink to dry, then dip the gelatine in water and float it on the glass in a little water. Note the gradual expansion of the gelatine as it absorbs water. After a time measure the increase in area of the gelatine strip by again placing the glass over squared paper. Now transfer the gelatine to a piece of fine muslin and hang it up to dry. It contracts, and gradually decreases in area owing to loss of water. The gelatine is a colloid gel.

(4) Put one drop of Indian Ink in a test tube and dilute with water till you can see through the mixture. Filter, and examine a drop of the filtrate under the high power of the microscope. It is full of fine particles. This is a coarse suspensoid sol. The particles show Brownian movement. The particles do not settle if the tube is left standing.

(5) Place a very little solid Congo Red in water. It apparently dissolves, the solution looks clear and the microscope reveals no particles. This is a fine suspensoid sol of which the disperse particles are ultramicroscopic.

(6) Examine the demonstration in which the three liquids (a) potassium bichromate solution, (b) Congo Red sol, (c) Indian Ink sol are illuminated laterally by a strong beam (Tyndall's beam). (a) is clear, $(b)$ and $(c)$ cloudy; $(b)$ looks more cloudy when thus lighted than when looked at against the light.

B. Difference in the Rates of Diffusion of Crystalloids AND Colloids.

(7) The two test tubes provided contain at the bottom orange and red jellies respectively. The orange jelly is impregnated 


\section{PHYSICAL CHARACTERS OF ORGANIC SUBSTANCES}

with potassium bichromate, the red with Congo Red. Half fill the tubes with water and view against a sheet of white paper. The potassium bichromate diffuses into the water quickly, the Congo Red very slowly.

(8) Another test tube contains jelly, the bottom layer of which contains sodium carbonate, while the top layer does not. Pour a little hydrochloric acid on the top of the jelly, it diffuses down through the substance. When the layer containing sodium carbonate is reached by the hydrochloric acid a reaction takes place, bubbles of carbon dioxide appearing in the jelly. [Note.The jelly must be made up immediately before the class, or the sodium carbonate will diffuse up into the top layer of jelly, and the reaction will take place immediately the hydrochloric acid enters the jelly.]

\section{Coagulation of Protein Colloid by Heat.}

(9) Place small portions of fresh white of egg in two test tubes. Add a little water to one : the water is absorbed by the colloid. Slowly heat the other: the white of egg sets to a solid. Cool and add a little water. The coagulated protein no longer absorbs water, having lost its colloid structure.

\section{Formation of Colloin Membrane.}

(Io) Carefully drop a crystal of copper acetate into a test tube containing a 2 per cent. solution of potassium ferrocyanide. A double decomposition occurs as the two salts meet and the copper ferrocyanide is precipitated to form a colloidal membrane around the crystal. This membrane enlarges by breaking and patching so that it seems to grow.

\section{E. Swelling of Vegetable Colloids in Water.}

(II) Cover with water on a sheet of glass the rectangular piece of seaweed [Chondrus is very suitable] provided, being careful to wet both sides. Note the expansion as with the sheet of gelatin in (3).

(12) Add water to a dried section of seaweed under the microscope and note the expansion that takes place.

(13) Compare the size and shape of dry peas with those of peas that have been soaked in water for 24 hours. Place six soaked and six unsoaked peas in rows touching one another: measure the aggregate diameter of the six in each case and determine the average absolute and percentage increase in the soaked peas. 


\section{PROTOPLASM AND THE AMEEBA. PROTOCOCCUS}

Constitution and Structure of Protoplasm. - The viscous but semi-liquid mobile protoplasm that forms the bulk of many animal and plant cells is to be regarded as a mixed colloid sol. The continuous phase is water in which various organic and inorganic substances are dissolved. These solutes may vary to a considerable extent according to circumstances. The disperse phase we believe to consist essentially of ultramicroscopic particles of protein, which may possibly include separate protein molecules, as well as aggregations of molecules. Fats or fat-like bodies may also exist as part of the disperse phase, and these may be closely associated with the protein droplets. In this sol numerous chemical and physical processes are constantly taking place, and it is the sum of these that give rise to the chemical phenomena of life.

Besides the ultramicroscopic disperse particles, which, together with the continuous watery phase, form, so far as we can tell, the ultimate structural basis of living protoplasm as a form of matter, the protoplasm ordinarily contains much larger microscopically visible granules and droplets of very various size and very various composition. The presence of these last, like that of the solutes, depends upon the state of the cell in regard to nutrition, etc. The sum total of the chemical processes 
going on in the living protoplasm is called metabolism, $\mathrm{I}$ and these larger granules and droplets are metabolites, i.e. products of metabolism. They must be carefully distinguished from the ultramicroscopic disperse particles which we cannot see, but whose existence we confidently infer from different lines of evidence, and which form the permanent structure of protoplasm.

In the clear protoplasm of the pseudopodium of the amœba (see Fig. I), in which there are no coarse granules, very numerous fine particles can be seen in Brownian movement. This movement is suddenly brought to an end by electrical stimulation. When the stimulation ceases the Brownian movement recommences. The mobile sol has been momentarily converted into a reversible gel. When protoplasm is killed by certain "poisons" (what are called in biology "fixing reagents," such as acetic acid, chromic acid or dilute iodine solu. tion, etc.) the sol is converted into an irreversible gel. If protoplasm is heated to $60^{\circ} \mathrm{C}$., however, chemical changes occur in the proteins, and the whole colloid structure is destroyed. The proteins are then said to be " coagulated."

The protein particles forming the disperse phase of the protoplasmic sol very readily run together to form a membrane (gel) in any plane on the two sides of which they are subjected to different physico-chemical action. This occurs for instance on the outer surface of a detached unit of protoplasm such as a cell, and this is accordingly covered with a semi-permeable protoplasmic membrane, the ectoplasm, which is relatively fixed and sharply distinguished from the rest of the cell protoplasm (endoplasm). Similar gel membranes are formed round the vacuoles (segregated drops of liquid enclosed

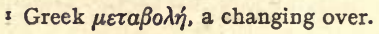


in the endoplasm), and also round the cell nucleus. It will be noted that all these phenomena of the living cell are strictly in accordance with what we learned of the behaviour of non-living colloids in the last chapter.

The special characters of protoplasm are largely dependent on the fact that it has the power of readily passing from the sol to the gel condition and back again. Normally it may be said to exist somewhere near the margin of the sol and the gel conditions. About $0^{\circ} \mathrm{C}$. it gels and becomes inactive, though as we shall see in later chapters it is not " killed," i.e. its essential structure is not destroyed, at far lower temperatures. As the temperature rises it becomes more labile and more active, till above $40^{\circ} \mathrm{C}$. changes begin to occur which eventually destroy its structure altogether. Many other causes besides changes of temperature and the loss or absorption of water send the protoplasm from the sol to the gel condition and back again, and it is on the changes in aggregation of the protein molecules and groups of molecules, the association and dissociation of these with one another, and with molecules of fats and salts, that what we call the vital activities of the protoplasm depend. The more aggregation occurs, the more gellike and relatively stable the structure of the protoplasm becomes, the less free the motion of particles and molecules from one part of the living cell to another. Consequently different chemical and physical processes can go on in different parts of the cell (as is often the case) without interfering with one another. Disaggregation leads to the opposite effect. Many of the more specialised animal cells have their protoplasm more or less permanently in the gel condition, and the specialised character of their activities depends largely on this fact. But on the whole the life activities 
depend essentially on the maintenance of the protoplasm within a comparatively narrow range of molecular aggregation, and on its power of passing backwards and forwards within this range.

Since the watery continuous phase of protoplasm is an essential part of its structure, the equilibrium of the protoplasmic water must be maintained or the structure of protoplasm would be destroyed. In other words, the forces tending to drive water out of the cell and those tending to draw water into the cell must be balanced. This condition of balance is maintained by primitive organisms living in water and consisting of naked protoplasm, when they are surrounded by water. If such an organism is transferred to air, evaporation from the surface into the air at once destroys the water balance, the body loses water and the organism dies. This is why terrestrial organisms which live surrounded by air must have some means of checking the loss of water by evaporation and of renewing it as it is lost. We shall see in later chapters that the history of evolution of land plants is very largely a history of the appearance and development of structures which have these results.

The Amœba.-The most suitable organism for preliminary study is the simple minute animal called the amœba. There are various species which live in freshwater pools and slow rivers, where they creep about upon the surface of the mud or upon the water plants.

Each individual consists of a minute irregular mass of naked protoplasm varying in different species from about I00 $\mu$ to about $250 \mu$ in diameter. ${ }^{I}$ The surface layer of the body (ectoplasm) is clear under the micro-

${ }^{x}$ I.e. from about $\frac{1}{10}$ to $\frac{2}{4}$ of a millimeter, or about $\frac{1}{2} \frac{1}{5 \sigma}$ to $\frac{1}{10}$ of an inch. 
scope, the rest of the protoplasm is filled with granules visible under the microscope and very variable in size and nature. Ir the ectoplasm, but bulging into the endoplasm, is situated a spherical space containing liquid, and called the contractile vacuole, because it can

A

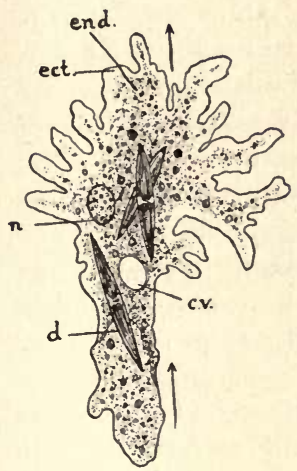

B

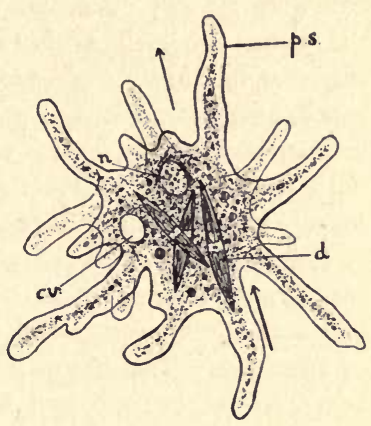

FIG. I.-A maba proteus. Two successive views of the same individual. The arrows show the direction of movement of the pseudopodia, which in $\mathbf{A}$ are adhering to the glass slide. ps., pseudopodium; ect., ectoplasm (clear); end., endoplasm (granular); $n$, nucleus: c.v., contractile vacuole; $d$, diatoms (unicellular plants), which have been taken into the endoplasm as food. The larger granules are the undigested remains of food. After Cash. $\times$ about 320 (the organism is just visible to the naked eye).

be observed to contract till it disappears completely or almost completely, and then quickly appears again in the same spot. The contractile vacuole is generally regarded as excretory in function, i.e. as forcing out of the body, whenit contracts, waste products of metabolism including urea (a waste product containing nitrogen), 
and perhaps also carbon dioxide. Thus its function is equivalent to that of the kidneys and bladder of the higher animals, and partly perhaps to that of the lungs.

In the endoplasm is a rounded body called the nucleus, which, as we shall see presently, is an all important part of the protoplasm. The rest of the protoplasm (ectoplasm and endoplasm together) is called cytoplasm. Nucleus and cytoplasm together form the structural unit of living organisation in the vast majority of plants and animals. Such a unit is called a cell, and the amœba is commonly called a unicellular animal because it consists of one such unit, in contrast with multicellular animals and plants which consist of many, in the largest forms of many millions.

The nucleus is covered with a nuclear membrane (gel membrane) and contains a substance chromatin, which can usually be seen under the microscope in the form of granules. The chromatin ${ }^{\mathrm{I}}$ is distinguished by its power of taking up and becoming coloured by dyes (stains), particularly certain stains (" nuclear stains"), with great readiness. This probably takes place by the adsorption of the particles of the dye by the disperse phase of the gel of which the chromatin consists. Chemically the chromatin is distinguished by the presence in it of complex proteins containing phosphorus.

The function of the nucleus in the life of the cell can be inferred from its behaviour and from the behaviour of the cytoplasm in relation to it under various conditions. Certain large Protozoa (unicellular animals) can be cut into pieces under the microscope without killing them. When this is done and one part of the divided cell contains the nucleus while the others do

1 Greek $\chi \rho \tilde{\omega} \mu a$, colour. 
not, the part containing the nucleus regenerates, i.e. it grows again into a complete cell. The parts not containing the nucleus remain for a time sensitive and motile, but they cannot assimilate food, and hence they ultimately die. In living plant cells, when the protoplasm is in a state of great activity and metabolism is proceeding rapidly, the nucleus is large and conspicuous (Fig. 43, A, B, C), but when the cell is comparatively inactive the nucleus is usually small and inconspicuous. Again, when local activity is going on in a cell the nucleus commonly moves to the spot in which work is being carried out. For instance, if a plant cell is thickening its cell wall in one spot the nucleus moves to the spot where the thickening is going on and remains there while this activity is in progress (Fig. 43, E). It appears to be "superintending operations."

From facts like these we infer that the nucleus is essential in directing the metabolic and formative cell processes. Exactly what it does we do not know, but very likely it sends out into the cytoplasm chemical substances, perhaps enzymes, which are essential in carrying out the work of the protoplasm.

The chromatin of the nucleus is certainly different in every species of organism, and even in those smaller groups of very uniform individuals within the species which are called "pure strains." The differences probably depend on the existence in the chromatin of slightly different proteins (cf. p. 43). The cytoplasm of different species and strains also probably differs in the same sort of way, but we have very good evidence that the differences of the chromatin are the most important. The peculiar formative powers of each species, the powers that enable it to reproduce the 
exact specific form of body and function, are certainly carried mainly, if not entirely, by the chromatin in reproduction, in which the nuclei of the reproductive cells play the leading part. Often indeed the nucleus of one of the parent cells of the new organism is practically the only thing contributed by that parent to the new organism, which may nevertheless exhibit some of the characteristics of that parent in a perfectly pure form.

Life Processes of Amœba.-The most obvious thing the amœba does is to move. When the animal is active its shape continually changes, the clear ectoplasm thrusts out from the body and is followed by the streaming granules of the endoplasm. The protoplasm of the projection so formed ( $p$ seudopodium) glides along the surface to which the amœba adheres, and may be followed by the streaming forward of the rest of the body, which thus gradually glides away from the position it occupied at first. Thus the animal constantly changes its shape ${ }^{I}$ and at the same time its position. Locomotion (movement from place to place) follows as an immediate result of the streaming movement of the protoplasm in a constant direction. The protrusion of pseudopodia is not, however, necessarily followed by locomotion: several pseudopodia may be put out at different spots on the surface of the body and again withdrawn, so that no continuous streaming in one direction occurs. Different species of amœba vary very considerably in the shape and dimensions of their pseudopodia.

The streaming movement of the pseudopodia is also one method by which the amœba feeds. When a pseudopodium touches another living organism much

$\checkmark$ The name amœba is derived from Greek $\dot{\alpha} \mu \circ \iota \beta \dot{\eta}$, change. 
smaller than the amœba, for instance a unicellular green plant (alga) or a colony of bacteria, the protoplasm flows round it or draws it into the body, so as completely to enclose the prey. With the prey a drop of water may be ingested, forming a food vacuole. The food is then acted upon by enzymes formed by the amœba's protoplasm, and the digestible parts digested, i.e. broken down into soluble organic substances, probably proteins, carbohydrates and fats, which can be assimilated by the amœba, i.e. built up again into new " amœba-protoplasm," while the indigestible parts are ejected from the body as faces. This process of defæcation is sometimes loosely called "excretion," but it must be carefully distinguished from the excretion of urea and carbon dioxide which are formed by the chemical breaking down of the proteins and carbohydrates of the body. The ingestion of the prey when the pseudopodium has once come into contact with it, and also the ejection of fæces, can be explained by the purely physical force of surface tension, for it has been shown that a drop of chloroform (representing the amœba) placed in contact with a minute fibre of glass covered with a varnish of shellac will ingest the fibre, remove the coating, and then eject the fibre (see Fig. 2). A drop of chloroform will also coil up a flexible fibre of shellac within itself just as an amœba will coil up a flexible algal thread (Fig. 3).

By the formation of new protoplasm the amœba naturally adds to its bulk and thus grows in size, unless of course the breaking down processes (katabolism) which result in excretion of the katabolites balance or exceed the building up or assimilative processes.

Amœba is also sensitive to external stimuli, i.e. its motion is directed in relation to such stimuli. For 
instance, its protoplasm streams away from a source of heat when the temperature rises above $35^{\circ} \mathrm{C}$. At $\mathrm{o}^{\circ} \mathrm{C}$. and at $35^{\circ} \mathrm{C}$, , or in the absence of sufficient dis-
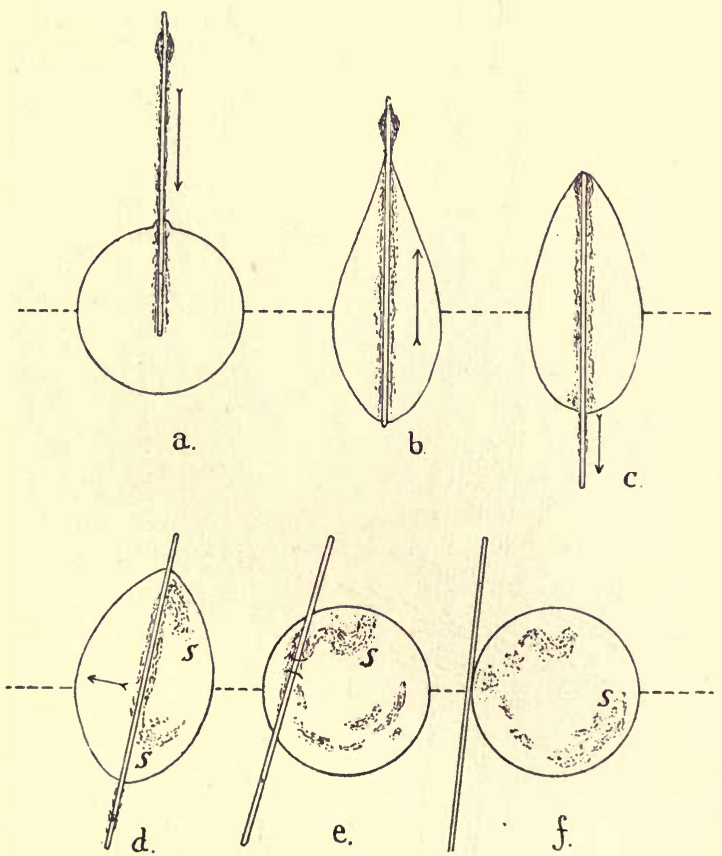

FrG. 2.-Spreading of a drop of chloroform over a shellac-covered glass fibre. Contact with the shellac lowers the surface tension of the drop of chloroform, which spreads over and draws the fibre into itself, removing the shellac. When the glass fibre is bare the chloroform recovers its surface tension, reassumes the spherical form and thus expels the fibre, which it cannot "wet." The arrows in $a, c$ and $d$ show direction of movement of the fibre; in $b$ the movement of the chloroform; $S$, shellac in the chloroform. After Rhumbler. 
solved oxygen in the water, the pseudopodia are withdrawn and the animal becomes spherical and motionless. Amœba is also sensitive to the presence of food, for it does not normally ingest objects which it cannot digest, and this probably means that chemical substances

A

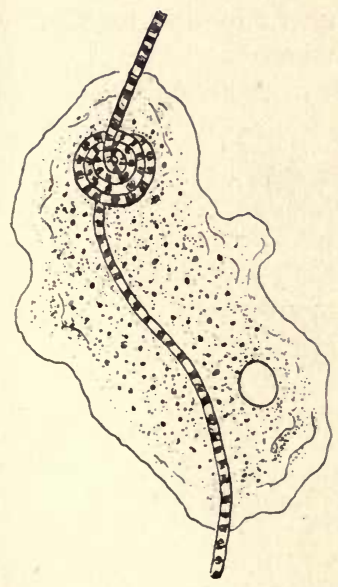

B

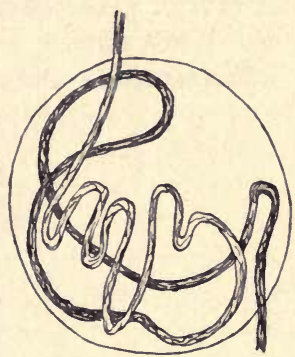

FIG. 3.-A, ingestion and coiling up of a thread of Oscillaria (an alga) by Amaba verrucosa. B, ingestion and coiling up of a thread of shellac by a drop of chloroform. After Rhumbler.

diffusing out from the organisms which form its prey come into contact with the ectoplasm of the amœba, and this stimulus leads to the envelopment of the prey.

Reproduction of Amœba.-When the growth of the individual amœba has reached a certain limit the animal 
divides into two. The nucleus first divides into two equal parts, and then a furrow appears in the cytoplasm and rapidly deepens till the two halves become entirely separate. This simple method of multiplication is called binary fission. Each of the halves is a complete amœba in every respect, and each proceeds to lead its own independent life and ultimately grows to the size of the parent amœba, when it divides again in the same way. [The amœba has other modes of reproduction which will not be dealt with here.]

Protococcus.-On the bark of trees, on old palings,

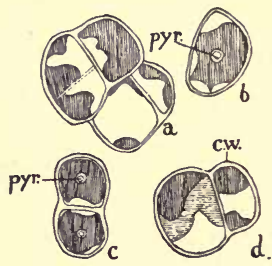

FIG. 4-Cells of Protococcus viridis. $\times$ about 800 . Each is bounded by a colourless cell wall $(c . w$.). The shaded portion is the green chloroplast which frequently occupies most of the space within the wall $(b$ and $c)$; pyr., pyrenoid. In $a$ and $d$ the cells have recently divided and are remaining together.

etc., especially on the north side, which does not encounter the direct rays of the sun and thus tends to remain damp, one often finds the surface covered with a thin green crust, which crumbles in dry weather under the point of a knife. If a little of this green crust is scraped off into a drop of water on a slide and examined first with the low and then with the high power, it is seen to consist of numerous very small green cells, which often hang together in groups of two, four, or more. This is a minute unicellular alga 
called Protococcus vulgaris, which inhabits the surface of the damp tree bark, and feeds by absorbing rain water that falls on the tree and trickles down over the surface. This rain water dissolves carbon dioxide from the air and small quantities of mineral salts, and thus it contains all the elements necessary for the food of the green alga.

Under the high power of the microscope (Fig. 4), each cell of Protococcus is seen to be bounded by a colourless cell wall of firm consistency. Inside this is the essential living part of the organism-the protoplasm of the cell. With the low power this appears uniformly green, but under the high power it can be observed that the green protoplasm does not fill the whole of the space within the cell wall, but takes the form of a curved plate (chloroplast) in which can often be observed a central shining body (pyrenoid), $x$ the rest of the cell being occupied by colourless cytoplasm. By appropriate staining the cell nucleus can also be distinguished, but it is generally hidden by the massive chloroplast.

Protococcus reproduces itself by cell division, the nucleus dividing first and then the chloroplast; a delicate wall is afterwards formed across the cell, separating it into two halves (Fig. 4, a, left-hand cell). The dividing cell wall thickens and the cells may separate, but very frequently they remain together in little groups. All stages of cell division and separation can frequently be seen.

If we compare Amøba and Protococcus as a representative simple animal and a representative simple green plant, we note first that they resemble one another

I A protein crystal-like body found in the chloroplasts of many green algæ and acting as a centre of starch formation. 
in both being isolated, independent, free-living organisms, each consisting essentially of cytoplasm with nucleus. But they show the following differences:-

(I) Amoba has no cell wall, while Protococcus has.

(2) Amoba moves from place to place, while Protococcus is stationary.

(3) Amoba feeds on living prey, while Protococcus does not, having no means of ingesting solid bodies.

On the other hand

(4) Protococcus has part of its cell protoplasm coloured green (chloroplast), which enables it to form organic substance, and eventually protoplasm, from liquid and gaseous inorganic substances which it can absorb through its cell wall. Amoba has no such power because it has no chloroplast.

\section{PRACTICAL WORK.}

\section{A. Amqeba as a Type of Simple Animal.}

(I) Place a drop of water containing the scrapings of the surface mud of a pond containing amœbæ (together with other organisms and organic débris) in the centre of a slide and cover with a coverslip. Examine first with the low power, and pick out the amœbæ, which appear as irregular blobs of greyish or brownish appearance. They will gradually creep out from the débris along the surface of the slide or coverslip.

Examine a specimen with the high power and notice that it is constantly changing its shape, sometimes slowly and sometimes rapidly. Distinguish the clear ectoplasm from the granular endoplasm, and note that the granules of the latter are very various in size and shape. The endoplasm may include quite large remains of ingested prey, e.g. the cell walls and disintegrated contents of unicellular plants. Note also the transient projections of the body (pseudopodia).

Identify the contractile vacuole, and watch it contracting and quickly reappearing at full size. Identify also the rounded, granular, light-refracting nucleus. Measure the average diameter of the amoba and the diameter of the nucleus with the eye-piece micrometer. 
(2) Draw an active specimen in successive stages of motion to show the change of shape depending on the protrusion and withdrawal of pseudopodia. See if you can observe an amœba in process of ingesting another organism as food.

(3) Kill the amœba by running a drop of iodine or acetic methyl green under the coverslip and note that the nucleus is stained more deeply than the cytoplasm.

(4) Examine a demonstration specimen of amœba which has been fixed, stained and permanently mounted, showing the deeply stained nucleus.

B. Protococcus as a Type of Simple Plant.

(I) Scrape off a little of the green crust from the bark of a tree into a drop of water and cover with a coverslip. Examine with the low power and note the numerous green specks and clumps in the field of the microscope. Put on the high power and distinguish cell wall, chloroplast, and colourless cytoplasm. The chloroplast generally contains a bright granule, the pyrenoid. More than one chloroplast may exist in the cell. The nucleus is usually difficult or impossible to distinguish. It lies in the colourless cytoplasm, often in the concavity of the curved chloroplast.

(2) Examine stages in the division of the cells (only found when the Protococcus is kept damp) (a) when the new cell wall is not yet formed but the chloroplasts of the daughter cells are distinct, and $(b)$ after the cross wall is formed but the two daughter cells are still clearly derived by division of the parent cell. Add a drop of very dilute iodine solution and observe the effect. 


\section{CHAPTER V}

\section{THE VITAL FUNCTIONS}

THE activities of a living organism which together make up what we call its "life" may be conveniently considered under separate heads which are often called "the vital functions." These divisions are to some extent artificial, for one process passes into another, and others are the results of the interaction of more than one. We must always remember that what we call the different life processes in reality form a continuous whole. They are all expressions of the activity of protoplasm in maintaining its physico-chemical equilibrium in relation to its surroundings, though these expressions are strikingly different in different organisms. The "vital functions" are those expressions which we can seize upon and give names to, and which are of essentially the same nature in all living organisms, whether animals or plants, simple or complex.

We must carefully distinguish between the activity of the organism as a whole and the activity of the protoplasm itself, though the former depends of course directly upon the latter, since protoplasm is the only living part of an organism. This distinction is least evident when the whole body of the organism, as in the amœba, consists solely of a naked mass of protoplasm. In multicellular organisms, the relations of the whole body to its surroundings are necessarily different from the relations of the protoplasm of each living cell to 
its surroundings. This we can illustrate in the case of each of the "vital functions."

(I) Feeding.-In the case of amœba, what we call the food of the animal is the living prey which it engulfs. The food of the protoplasm, however, consists of the products of digestion of the prey which can be directly incorporated in the protoplasm, or can be broken down to set free energy without such incorporation. Similarly, in the case of a higher animal, the food of the animal is the animal or plant, or the part or product of the animal or plant, which the animal eats; the food of the protoplasm of its living cells consists of the digested products of the original food which have passed into the blood and can be absorbed and incorporated into their structure by the living cells, or broken down into simpler substances so as to liberate energy.

In a green plant the food of the plant as a whole consists of the water and inorganic salts absorbed by the roots from the soil, and of the carbon dioxide absorbed by the leaves from the air. The food of the living protoplasm of the plant cells, on the other hand, consists of the sugars and proteins built up from these simple substances taken in by the plant. In the case of a germinating seed the food is obtained directly from reserve stores-starch or fats, which are converted into sugar, and proteins which are converted into a soluble form-that are packed away in the seed. Here, it will be noted, the foods of the young plant are of exactly the same chemical nature as the foods of an animal, and they can be used, and are in fact largely used, as food by animals, as when we eat bread made from the reserve stores of the wheat seed, or beans, peas or nuts, which are also seeds with large stores of food.

Thus, the food of the organism as a whole is very 
different in different kinds of organism, though it must always contain the necessary chemical elements, but the food of protoplasm is always of the same nature.

Four main types of feeding are often distinguished: holozoic, in which the organism ingests solid organic food (characteristic of animals); holophytic, in which the food of the organism consists wholly of inorganic substances, including carbon dioxide (characteristic of green plants); saprophytic, in which organic food is obtained from the dead body or from the products of some other organism in a liquid form; and parasitic, in which organic food is obtained direct from the living body of some other organism, but this " host " organism is not immediately killed. The mode of nutrition of saprophytic and parasitic organisms is not essentially different. It is of no essential importance whether the liquid organic substances, provided they are suitable as food, come from a living or from a dead body or product; and many species of fungi, for instance, may be both saprophytic and parasitic (see Chapter XI), though in many cases species are closely adapted to one or the other mode of life. Animal parasites may have mouths, and suck or devour organic liquids or tissues of the living body of the host, or they may, like the tapeworm, have no mouths, and absorb liquid organic food through their body wall.

(2) Assimilation. I-This word literally means " making like," and in its strict sense is applied to the process of incorporation of the final foodstuffs in the specific protoplasm of the organism. It is, in fact, the actual feeding of the protoplasm. We know practically nothing of how this is done The incorporation of the carbon derived from the carbon dioxide of the air in

Latin similis, like. 
the molecule of sugar, the first stage in the formation of the protein molecule that goes to form the structure of protoplasm, is often called " carbon assimilation."

Anabolism and Katabolism.-All the constructive chemical processes which take place in the protoplasm are classed together as anabolic I processes or anabolism, as opposed to the destructive or katabolic ${ }^{2}$ processes (katabolism). Thus the formation of sugar from carbon dioxide and water in a green plant is an anabolic process, the breaking down of sugar, and, in the animal body, the breaking down of proteins into urea and uric acid, are katabolic processes. Assimilation in the strict sense, i.e. the incorporation of foodstuff in the protoplasm, is the ultimate anabolic process.

(3) Growth.-In its simplest form growth follows directly from assimilation, from the incorporation of new material which increases the mass of the protoplasm. Unless this new protoplasm is destroyed as quickly as or more quickly than it is formed, increase in bulk must result. In amœba the animal simply increases in size till it reaches a certain limit, when division takes place, and the two daughter amœbæ go on feeding and growing independently. In more complex organisms of definite shape growth takes very various forms in different parts of the body, but it always leads to permanent increase in the bulk of the organism-a temporary inflation of part of the body, for example, is not a process of growth.

The higher plants differ widely from the higher animals, in showing localised growth, in most cases at the ends of the branches of the shoot and of the root, leading to increase in length of the branches, and this may be more or less continuous throughout s Greek ảvá, up.

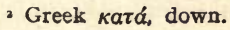


the life of the plant. In addition to this increase in length of the branches, definite layers of cells inside the plant may also grow, increasing the thickness of the stem and root, as happens in the case of trees.

Most animals have a definite growth phase in youth, which involves the whole of the body, but is different in nature, amount and duration in the different organs. Thus a child's trunk and limbs grow more, from birth to adult life, than its head, and similar differences are shown in the growth of the different internal organs.

Both in animals and plants it is clear that the growth of an individual living cell is a different thing from the growth of the organism as a whole, though the latter depends upon the growth and division of the cells.

(4) Differentiation.-A multicellular organism arises, during its individual lifetime, from a single cell. This cell divides, and the daughter cells, instead of separating from one another, as they do after cell division in. amœba, remain together, and again divide, the new daughter cells behaving in the same way. In this manner the adult multicellular body is produced. But the cells of this body are not all alike; sets of them are grouped together into tissues and organs, and the cells of one tissue differ widely from those of another in form, structure and function. The process of becoming different is called differentiation, and accompanies the growth of the bodies of all multicellular organisms. Thus growth depends upon the two processes, cell division and cell differentiation.

The protoplasm of a single cell may be differentiated. Thus in the Protococcus cell the chloroplast is differentiated from the colourless cytoplasm, whereas in amœba there is no such differentiation. In some unicellular animals the differentiation reaches a high pitch, the structure of the single cell being very complicated. 
But such intracellular differentiation ${ }^{\mathrm{x}}$ has limits, because a cell is unworkable if it exceeds a certain size, and the elaborate differentiation of the higher organisms depends upon their multicellular structure.

(5) Respiration.-The functions hitherto considered are all concerned with building up the protoplasm of the organism. We have now to consider processes connected with the expenditure of energy by the organism and with the breaking down of organic substances.

When the amœba moves it does work and spends energy in doing it. This energy is derived from the energy locked up (potential energy) in the molecules of organic substance taken in as food-largely in the carbohydrates. The niolecules of these substances are broken down into simpler substances and largely oxidised in the process, the energy being set free and appearing in the kinetic form, i.e. as mass motionfor instance, the streaming of the amœba cytoplasmand heat. Free oxygen is necessary to carry out this process, and that is why free oxygen is required by very nearly all living beings. Aquatic organisms get their oxygen from that which is dissolved in the water they live in, terrestrial forms direct from the air. In its absence the activity of the organism comes to an end.

The release of energy is obtained almost entirely by the oxidation of sugar (glucose) with the formation of carbon dioxide and water.

The generalised equation is :-

$$
\begin{aligned}
& \mathrm{C}_{6} \mathrm{H}_{12} \mathrm{O}_{6}+6 \mathrm{O}_{2}=6 \mathrm{CO}_{2}+6 \mathrm{H}_{2} \mathrm{O} \\
& \text { glucose oxygen carbon water } \\
& \text { potential energy } \\
& \text { dioxide } \\
& \rightarrow \text { kinetic energy } \\
& \text { (mass motion and heat) }
\end{aligned}
$$

This process is what is called in biology respiration.

I Differentiation within the cell, from Latin intra, within. 
The same chemical process (oxidation) takes place when we burn sugar in the air, the same products, carbon dioxide and water, and kinetic energy in the form of heat, resulting. When we burn coal or petrol in an engine a similar process takes place. Here the kinetic energy is partly used to drive a machine, for instance a steam locomotive or a motor car, and this mass motion is comparable with the mass motion of protoplasm. In both cases some of the energy appears as heat, raising the temperature of the cell or of the engine, as the case may be.

The living protoplasm of all plants requires free oxygen for respiration just like that of animals, though plants do not use it so quickly as active animals do. In green plants in light respiration is masked, because, as we shall see in a later chapter, the opposite processthe formation of sugar from carbon dioxide and water, with production of free oxygen-is carried out at a greater rate than respiration. In plants (and in parts of plants) that are not green and are growing quickly, i.e. expending much energy, for example in germinating seeds and opening flower buds, a great quantity of oxygen is used, and has to be taken from the air, and a great deal of carbon dioxide and heat are produced. "Breathing" in the higher animals, also called " respiration," I consists of the rhythmically alternating processes of "inspiration" and "expiration." The former is the taking of air into the lungs so that oxygen may be absorbed through the walls of the lung tissue by the hæmoglobin of the red blood corpuscles, and carried by them in the blood stream to all the tissues of all the organs of the body, where respiration in the

* This of course is the original meaning of the term (from Latin spiro, breathe). 
general biological sense takes place in the living cells. The return blood stream comes back to the lungs laden with carbon dioxide, the result of the tissue respiration, and this gas, diffusing into the air passages, passes out of the body in the "expired" air. Here again we get the contrast between the function of the organism as a whole, in this case carried out by special organsthe lungs-and the function of the individual cells of which it is composed. It is especially in the muscles that sugar is most actively broken down in the bodies of the higher animals, and accordingly it is here that the greatest amount of kinetic energy is set free, as we see in the vigorous contractions of our muscles.

Plants have no "organs of respiration" comparable with the lungs of the higher vertebrate animals. The oxygen used in respiration by the living cells of plants diffuses into the plant from the air through the system of air spaces between the cells (intercellular spaces), and so through the water saturating the wet cell walls into the protoplasm. The oxygen actually used by the living cells in respiration, both in animals and plants, is always oxygen dissolved in liquid, though it is ultimately derived from the air.

(6) Katabolism and (7) Excretion.-Respiration is essentially a katabolic process, involving the breaking down of an organic substance (sugar) in the living cell. But animals have, in addition, a nitrogenous katabolism involving the breaking down of proteins. This process goes on largely, though not wholly, in the liver, and the comparatively simple nitrogenous substances formed in the liver pass into the blood and are excreted through the kidneys as urea $\left(\mathrm{CH}_{4} \mathrm{~N}_{2} \mathrm{O}\right)$ and uric acid $\left(\mathrm{C}_{5} \mathrm{H}_{4} \mathrm{~N}_{4} \mathrm{O}_{3}\right)$, and pass out of the body in the urine. In the amœba these nitrogenous excretions are probably expelled 
through the contractile vacuole. It will be noted that these substances are not nearly so fully oxidised as $\mathrm{CO}_{2}$, i.e. oxygen forms a much smaller proportion of the molecule, and the animal derives a negligible quantity of its energy from the breaking down of proteins. Plants have no comparable nitrogenous katabolism. If katabolites are formed from the breaking down of proteins they are not systematically excreted, but are probably often used again for the formation of proteins. Plants are, in fact, much more economical of their nitrogen than are animals. Various substances, some of them containing nitrogen, may be cast off by plants, as in the bark of trees, while carbon dioxide regularly diffuses out of the tissues that are not green, and from all tissues in darkness, but there is in plants no specialised excretory system.

(8) Movement.-This is the most obvious of all the expressions of life. We can again distinguish between the movement of the protoplasm of living cells such as we see in the streaming of the protoplasm in amœba and the movement of parts (for instance the limbs, involving whole organs and systems of tissues in the higher animals), or the movement of the whole organism from place to place (locomotion). In amœba we can observe under the microscope how the first leads directly to the second (formation of pseudopodia), and this again to the third. In a higher animal the same connexion occurs, but the chain of causation is much longer. The movements of parts, for instance the limbs, depend on the contraction of the living substance of highly specialised tissues, the muscles; through the definite relationship of these with the bones the movements of several muscles, co-ordinated through the agency of the central nervous system, leads to the movement of 
a limb; while the co-ordinated movements of the limbs may result in the movement of the body from place to place.

In the cells of plants the rapid streaming movement of the cytoplasm, which in certain cases may be observed under the microscope to stream round the cell, is perhaps always the result of an injury or other shock: there is no evidence that it occurs normally in the untouched plant. Nevertheless it provides conspicuous proof of the power of plant protoplasm to move comparatively rapidly. There is also plenty of evidence of the power of rapid movement of plant cells under normal conditions, e.g. in the motility of many of the lower unicellular plants and of the reproductive cells in many fixed plants, though these move by means of the contraction of special delicate cytoplasmic processes projecting from the cell body (cilia and fagella) whose rapid beating pulls or pushes the organism through the surrounding liquid. Many unicellular animals also move in this way.

The commonest form of movement of the protoplasm of plant cells is, however, a slow streaming movement, usually too slow to observe directly, but evidenced by the frequent changes in place of the constituents of the living cell body, such as the nucleus, and in green cells, the chloroplasts. The growth of a plant clearly involves the result of a multitude of slow movements, and all of these, like the much quicker movements of animals, depend upon a constant supply of energy which is derived from the release of potential energy by the breaking down of organic molecules through oxidation. A moment's reflection will convince us of the large amount of energy involved in the building up and raising of the branches of a tree, eventually weighing perhaps many tons, high into the air against the force 
of gravity, and in the burrowing of the roots far into the substance of resistant soil.

(9) Response to Stimuli.-All living protoplasm responds to various stimuli in various ways, or in other words it is sensitive to the forces acting upon it from its surroundings. This response depends upon the setting up of processes within the protoplasm which result in some change in the activity of the latter. The most conspicuous expression of response to stimulus is a movement. It is necessary to be clear as to the difference between this kind of movement and the merely passive movement which results from the application of an external force. For instance, if one man pushes another over the edge of a cliff, the falling of the victim is a passive movement, resulting from the force of the push and the force of gravity. But if the intended victim, after receiving the push, saves himself by a sudden leap to one side, that movement is a response to the stimulus of the push. It involves a change in the activity of his nerve and muscle cells. This is a very complicated response in a highly complex organism, though it is not nearly so complicated as the responses we carry out every minute in the course of our daily lives.

The very simplest organisms, however, exhibit responses to various stimuli, and these are specific, i.e. they vary quite definitely according to the stimulus. It is probable that they can all be explained as consisting of a series of purely physical and chemical processes within the protoplasm, set in motion by the stimulus; as for instance in the case of the ingestion of food by the protoplasm of the amœba, which, as we saw, is closely paralleled by the ingestion and ejection of a glass splinter coated with shellac by a drop of 
chloroform. But in most cases we do not as yet know nearly enough of the chemistry and physics of the living cell to furnish a detailed explanation of the causes and course of the particular response in every case.

When the whole organism moves in response to an external stimulus, the motion is called a taxis. ${ }^{\mathrm{I}}$ When, as in a fixed plant, an organ bends in response to a stimulus, the motion is called a tropism..$^{2}$ For instance, motile unicellular green plants (e.g. Chlamydomonas, Chapter XII) are positively phototactic 3 in light of weak or medium intensity coming from one side i.e. they move towards the source of such light. Motile unicellular organisms in general are chemotactic, either positively or negatively, i.e. they move towards or away from the source of various chemical substances diffusing from a given source, according to the nature of the substance. The tips of the branches of the stem and root of a higher plant are phototropic, geotropic 4 and hydrotropic 5 in different cases, bending towards or away from a source of light, the direction in which gravity is acting, or a source of moisture. Thus the tips of roots are positively geotropic and hydrotropic, but negatively phototropic, and so on. Of the detailed effects of other stimuli, e.g. electrical stimuli, on the protoplasm of cells we do not know enough to speak confidently, though we know that such effects exist.

(Io) Reproduction may be defined as the production of a new individual or new individuals from pre-existing ones. It has been usefully defined as discontinuous growth, since the formation of a new individual always involves processes of growth, and this growth is discontinuous, for it begins in the parent organism

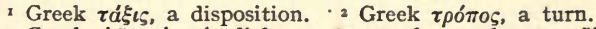

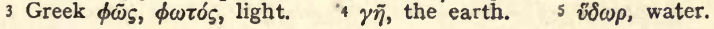


and is carried on in the separate new organism, or organisms, which is, or are, the offspring.

The simplest form of reproduction is binary fission such as we saw in the amœba, the division of the body of the parent into the bodies of the offspring. We do not completely understand the forces which set this process at work. All we can say is that it seems to be ultimately determined by a size limit corresponding with a limit of the surface/bulk ratio, which decreases as the organism grows in size. We know that the surface of a sphere increases as the square of the radius, while its volume increases as the cube of the radius. Thus a unicellular organism, as it grows in size, will have a smaller and smaller surface in proportion to its volume, and this will have an important effect on the processes going on within the protoplasm. The supply of oxygen, for instance, which has to pass through the surface from the surrounding water will be progressively less for each unit volume of protoplasm the larger the organism becomes. It is probable that some effect of this nature initiates the process of division.

A large number of unicellular organisms are reproduced by binary fission or by some simple modification of it. But multicellular organisms produce special reproductive cells which are separated from the bodies of the parents and produce new organisms (offspring) by growth and differentiation, which may begin and be carried on for some distance (in the highest organisms to the greatest extent) before complete separation occurs. In Chapter XII we shall consider the relation of these special reproductive cells to the individual unicellular organism which reproduces itself by binary fission.

Reproduction may also take place, as in most plants 
and in some of the lower multicellular animals, by the separation of parts of the body which are not special reproductive cells, e.g. by the separation of some segments of the body in worms or by the rooting of cuttings and "layers" of plants. This formation of new individuals from non-specialised or only slightly specialised parts of the ordinary body of the organism is called vegetative reproduction. It does not occur in the highest animals.

\section{PRACTICAL WORK.}

\section{A. Respiration and Production of Heat in Living Seeds AND Flower Buds.}

(I) The test tube provided contains living barley grains (seeds) which have been wetted and placed in the test tube, which was corked on the previous day. They absorb water and swell as a preliminary to sprouting. They also absorb oxygen and give off carbon dioxide in considerable quantity as a result of the rapid respiration of their living cells, which resume active life as a result of absorption of water and oxygen. Test for the carbon dioxide by dipping the rod into lime water, carefully uncorking the test tube and inserting the tip of the rod close to the grains without touching them. After thirty seconds or so look at the drop of lime water on the rod against a dark background.

(2) Examine the demonstration consisting of a $\mathrm{U}$-tube containing coloured water and connecting with two corked flasks, one containing wetted living and the other wetted dead barley grains. Fixed to the cork inside each flask is a lump of caustic potash which readily absorbs carbon dioxide. In the flask containing living grains oxygen has been absorbed by the grains while the corresponding carbon dioxide produced is absorbed by the caustic potash. In the flask containing dead grains no such change has occurred. Hence the total amount of free gas in the two flasks changes and the pressure on the surface of the liquid in the two limbs of the $\mathrm{U}$-tube becomes unequal.

(3) Examine the demonstration showing that the temperature rises in the middle of a mass of wetted living barley grains and not in the corresponding mass of dead ones.

(4) Examine the demonstration showing the rise of tempera- 
ture of chrysanthemum (or other suitable) buds in a vacuum (thermos) flask. Here the evolution of heat is "cumulative" for a time because the heat cannot escape, and the rising temperature accelerates the process of respiration.

\section{B. Localisation of Growth in Plants. Response to Stimulus.}

(I) Take two beans which have germinated and produced roots from one to two inches long. If the surface of the root is wet, dry it carefully with a piece of torn filter paper, taking great care not to break or injure the root. With a carefully sharpened stick (or lead pencil) dipped in Indian ink make marks on the root at intervals of one-tenth of an inch from the top to the seed. Now push a large pin carefully through each bean, and fasten to the under side of the cork of a wide-mouthed bottle, so that when the cork is replaced the root of one points vertically downwards while that of the other is horizontal. Pour a little water into the bottom of the bottle, replace the cork with the beans pinned to it and turn the bottle upside down for a few seconds, so as to wet the beans and provide water for their growth. Leave the bottles till next time.

(2) Examine the demonstration showing the aggregation of the green motile unicellular plants (Euglena) on the glass side of a jar. The Euglenæ were scattered through the water in the jar, which was covered with black paper. The only light reaching the Euglenæ came through the stencil holes in the paper, and they moved towards it, eventually adhering to the glass under the openings.

\section{Organic Foodstuffs of Plants and Some of their Colour Reactions.}

Representatives of the three great classes of organic foodstuffs-proteins, carbohydrates and fats-are stored in seeds and other storage organs such as tubers, and are used to feed the young plant arising from the seed or tuber before it is able to feed itself. These foodstuffs can be identified under the microscope, partly by their appearance, partly by the use of different stains and reagents :-

(I) With watery solution of iodine in potassium iodide (commonly called "iodine solution ") grains of proteins turn yellow or yellow brown, starch blue, cellulose and fats remain uncoloured. 
(2) With solution of zinc chloride in potassium iodide ("Schulze's solution ") proteins turn yellow as before, starch blue, cellulose blue or purple, fats remain uncoloured.

(3) With Sudan 3 (an anilin dye) proteins, starch and fats remain uncoloured, fats turn orange-red.

Stain an example of each of the three sections of different seeds : (a) bean, (b) castor oil plant (Ricinus), (c) lupin, in watchglasses containing the reagents (I), (2), (3) above. Wash in water and mount in dilute glycerine. Draw a single suitable cell of each section under the high power, and determine the classes of food substance present in each. 


\section{THE CELL}

WE have now become acquainted with two simple living organisms, Amoba and Protococcus, with their structure, behaviour and life histories; and we have seen that the "vital functions" which they exhibit are expressions of the activity of the protoplasm of which their bodies are composed, and that these same " vital functions " are exhibited by all living organisms, however large and complex, however different in structure and appearance-by trees and human beings, just as by Protococcus and Amceba. But we have also seen that the exact ways in which the various functions are performed by the organism as a whole varies with its structure, and before we can understand these variations in any detail we must become acquainted with the organisation and variations of the unit of which organic structure is built up-the cell.

Unicellular and Multicellular Organisms. Differentiation and Division of Labour. Tissues.-We have already seen that the whole body of an amœba is a unit of living protoplasm consisting of cytoplasm and nucleus: the same is true of Protococcus, which has, however, a special green protoplasmic body called the chloroplast within the cell which enables the organism to make carbohydrate out of carbon dioxide and water, and also a non-living cell wall of cellulose (a complex carbohydrate) which is formed by the protoplasm. 
These organisms are called unicellular, because they consist of one such unit, in contrast with the great majority of plants and animals which are composed of many such units, each with its own cytoplasm and nucleus-which are in fact multicellular.

The unicellular organism may show a considerable differentiation of parts within the cell. The primary differentiation into nucleus and cytoplasm is found in all (except, perhaps, the bacteria, see Chapter IX), but in Protococcus we find the differentiation, characteristic of all green plant cells, of the cytoplasm into the chloroplast and colourless cytoplasm. Many of the higher Protozoa have quite complicated cells. For instance the cell may have a firm cortical cytoplasm (gel structure) which maintains the shape of the body, a definite opening (mouth and gullet) through this leading into the central more motile cytoplasm (sol), as well as numerous exceedingly fine threads of cytoplasm (cilia) which project from the surface of the body, and by their rapid beating pull the animal quickly through the water. Thus we have different parts of the cell, differentiated and performing different functions for the whole, e.g. the mouth and gullet for feeding, the cilia for motion, etc. Other kinds of Protozoa have skeletons of non-living substance, enclosing the protoplasm which projects through openings in the skeleton.

Though this differentiation is often carried much further in a unicellular organism like a Protozoon than in the individual cells of a multicellular organism, the differentiation between different cells which is possible in a multicellular organism is much greater, because there is no limit to the size of the organism. In the simpler multicellular plants (e.g. Green Algæ) there is 
little or no differentiation between different cells, but in the higher plants it is considerable.

A set of similar cells which perform one special function or set of functions in the organism is called a tissue. ${ }^{I}$ Thus in the higher plants there are absorbing tissues, conducting tissues, sugar-forming tissues, protective tissues, etc. Specialisation of different cells for different functions reaches a far higher pitch in the higher animals, where different tissues are associated in the different organs, and work together to perform the special function of the organ, e.g. lungs, heart, liver, kidneys, etc.

However specialised the structure and work of a cell may be, it must necessarily be able to carry out the vital functions described in the last chapter, or it would not be alive. It depends, however, upon its position in the body and its relation to other cells and tissues for the essentials of its continued existenceit cannot lead a free independent life like the cell of a Protozoon and obtain its food and oxygen directly from outside the organism. Thus the muscle cells depend for their food and oxygen on the circulation of the blood which carries these necessary substances to them. Each highly differentiated cell or tissue specialises on one function of protoplasm, which represents its particular work for the organism as a whole. Thus the fibres of the muscle tissue (Fig. 5, F) show the power of contracting-a specialised form of movement-in a very high degree, and by means of the co-ordination of the contractions of many muscles the organism moves. The cells of the glands attached to the alimentary canal specialise in the production of enzymes which they pour out into the cavity of the gut for

3 From the analogy of its texture with that of a textile fabric. 
purposes of digestion of the food of the whole organism. Some of the gland cells lining the air passages are ciliated and others produce mucus (Fig. 5, B).
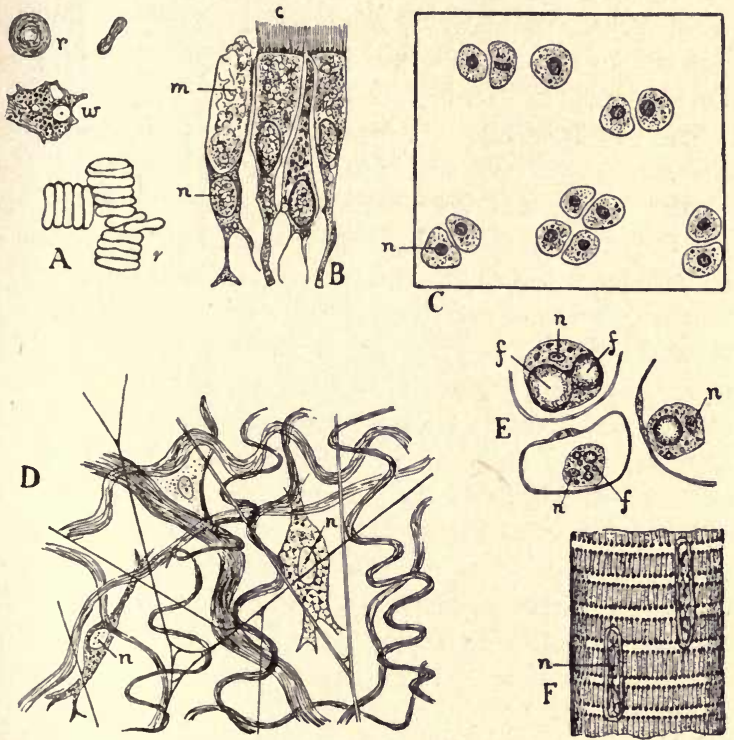

Fig. 5.-Cells from different tissues of a higher vertebrate animal. A, blood corpuscles; $r$, red corpuscles-these are not complete cells and have no nuclei; $w$, white corpuscle, whose structure and movements are very similar to those of amoba. B, cells of the ciliated glandular epithelium lining the trachea (air tube to lungs); $n$, nucleus; $m$, mucus being expelled from the cell; $c$, cilia. C, cells of cartilage recently divided, embedded in cartilaginous matrix secreted by the cells; $n$, nucleus. $\mathrm{D}$, cells and fibres of connective tissue (which forms the "packing " of the organs of the body); $n$, nucleus. $\mathrm{E}$, fat-forming cells ; $f$, globules of fat; $n$, nucleus, F, Part of striped muscle fibre. Here the protoplasm is highly modified into special contractile substance; $n$, nucleus: many nuclei in each fibre, which is composed of many fused cells. The nuclei are situated just below the very thin outer membrane. 
The white blood corpuscles, on the other hand, are unspecialised cells, very much like amœbæ (Fig. 5, A, w). Highly specialised cells lose the power of multiplying by division as soon as the tissue to which they belong is fully differentiated. In general the power of reproduction by division is the function of an unspecialised cell.

The Cell Doctrine.-Every multicellular organism begins life as a single cell, which is separated from the body of the parent and by cell division, growth, and (in all but the simplest forms) by differentiation of the products of division gives rise to the body of the adult offspring. In this process of cell division the daughter cells do not separate from one another, as in the binary fission of a unicellular organism, but remain together, and in all the higher forms undergo differentiation into tissues and organs to form the different parts of the body.

This is the basis of the generalisation-known as the cell doctrine-that organisms consist of cells, which are the structural units of the organism, and that the functions of the organism as a whole are made up of the sum of the functions of all its cells. Broadly speaking this is true, but certain qualifications have to be made.

In the first place, in some of the lower forms the body does not consist of distinct cells, though it contains a great many nuclei. Thus in some algæ and fungi among the plants the body is composed of a branched tube of cellulose (or similar substance) enclosing a continuous mass of cytoplasm in which are scattered numerous nuclei. As the plant feeds and grows the protoplasm at the tips of the branches increases and the nuclei embedded in it increase in number by division, the wall covering the tip continually having new 
layers added to it by the protoplasm within, and being pushed out as the tube grows in length. Here there is no cell structure, and such plants are called noncellular. Also there are parts of animals, and phases in the development of animals, where there is no clear cell structure, but for instance a network of cytoplasm with a nucleus at each "node" of the network, i.e. at each thickening of the cytoplasm where several branches of the network meet. This kind of structure does not however differ essentially so far as its working is concerned from ordinary cell structure. We have already seen (p. 66) that the nucleus is essential to the carrying out of the vital functions of the protoplasm; and in these cases of non-cellular or incomplete cellular structure we must suppose that each nucleus governs, so to speak, a certain sphere of cytoplasm around it, though this sphere is not sharply separated, as it is in ordinary cellular structure, from the neighbouring spheres or units. Such a working unit of nucleus and cytoplasm has been called an energid. An ordinary living cell represents one such energid, but in noncellular living structures the energids are not separated by clear boundaries.

The second qualification of the cell doctrine that has to be made relates to the unity or individuality of the multicellular organism. To some degree in all such organisms, and very notably in the higher animals with a central nervous system, the activities of each cell are modified by its connexion with the other cells of the body, so that the organism works as a whole. In this sense, as we saw in the last chapter, the organism as a whole has functions which are something distinct from the functions of the individual cells or energids of which it is composed, though these are built up as 
a result of the sum total of the functions of all its cells or energids.

Cell Walls and Intercellular Substance.-In animals the substance of the body does not necessarily consist entirely of the actual bodies of living cells, though the whole of the substance is formed by the activities of these. The cells themselves frequently lie in a " matrix" of intercellular substance which has been formed by the cells which were originally close together but eventually lie singly or in groups scattered in this matrix. This is very clearly seen in the case of cartilage (Fig. 5, C) and bone (which is developed from cartilage by changes in the matrix). In the case of connective tissue, the tissue which forms the "packing" of the organs of the body, the matrix is differentiated into two kinds of fibres which run through it, singly or in bundles, independently of the connective tissue cells (Fig. 5, D). In the case of muscle the actual cytoplasm of the original cells is modified into a peculiar substance which shows no cell structure, but is composed of fibres that have an enormous power of contractility. The cell nuclei still remain lying below the membrane enclosing the muscle fibre (Fig. 5, F). Some animal tissues have a distinct cell wall surrounding the cytoplasm of each cell. This may be composed of modified cytoplasm or of simpler organic substances, usually containing nitrogen.

Plants, on the other hand, very rarely have " intercellular substance." So much of the substance of the plant body as is not actually composed of the living bodies of cells is made up of actual cell walls, which in the adult state are still clearly seen to have been formed by the cells, and the basis of these walls is generally cellulose. The cell walls of a plant form the 
skeleton or framework of the plant body, and in the higher plants a considerable part of the structure (in trees the great bulk of the structure) is composed of the walls of cells which have died, so that only the wall is left. These walls are often very thick and hard, growth in thickness and the deposition of substances other than cellulose having taken place before the protoplasm of the cell died and disappeared. Another feature of the tissues of the higher plants (land plants) is the existence of a system of air spaces (intercellular spaces) between the cells. These are made by the separation of the walls of certain adjacent cells from one another, and penetrate the plant, communicating with the outer air and forming the channels by which gases, such as water vapour, carbon dioxide and oxygen, produced or used by the living cells, diffuse from these to the air outside, or vice versa.

\section{The Plant Cell.}

The first cells of organisms to be described were those of the cork tissue of plants, which were seen by the Englishman Hooke in 1667 with the compound microscope that was devised about the middle of the seventeenth century. Hooke compared these cork cells with the "cells" of honeycomb. Thus it was the walls enclosing cavities to which the term was first applied. Little accurate knowledge of the contents of these cavities was obtained for nearly two centuries. But in I83I the English botanist, Robert Brown, discovered the cell nucleus, and in 1846 the German botanist, von Mohl, identified the semi-transparent viscous substance formed within many of the "cells " of the adult higher plant as the actual living substance of the organism, 

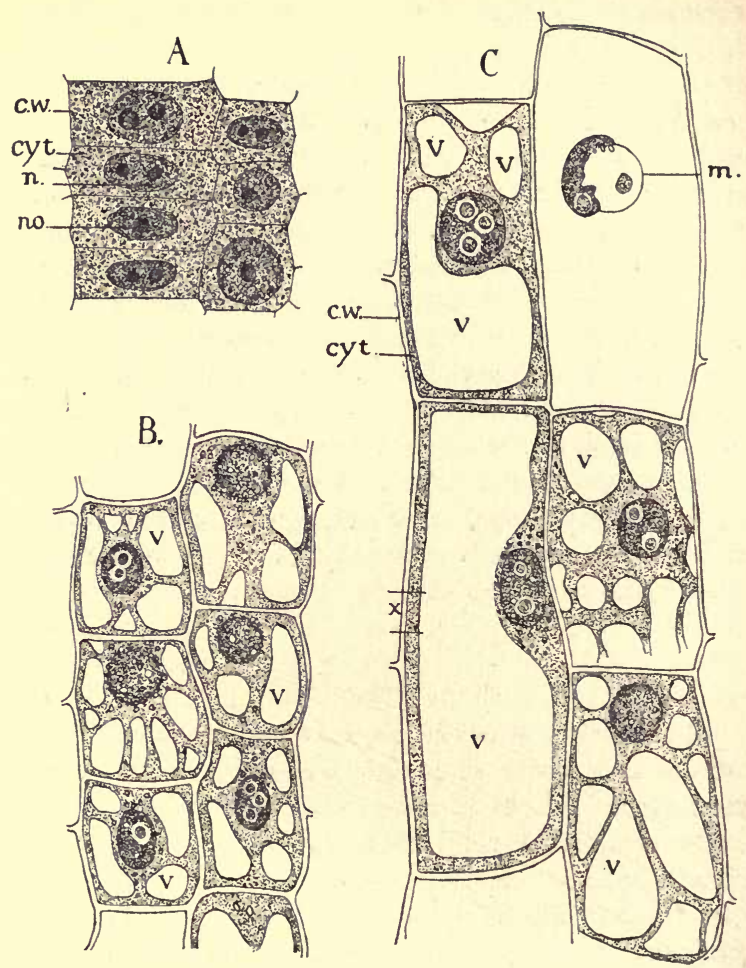

FIG. 6.-A, embryonic cells from the meristem (growing point) of the root. $B$, beginning of vacuolation. $C$, cells from elongating region with larger vacuoles; c.w., cell wall; cyt., cytoplasm; $n$, nucleus; no., nucleolus; $v$, vacuole. The top right-hand cell in $\mathrm{C}$ has been cut and water admitted to the cavity; the nucleus has absorbed water and burst, a new gel membrane $(m)$ being formed on the surface of the escaped contents in contact with the water. $\times 660$ (after Sachs). 
and called it protoplasm I This term was then extended to the essentially similar substance found in animals. The parallelism between the units of protoplasm, each with its nucleus, in animals and plants was soon recognised, and the term cell gradually came to be used in its modern sense as applying primarily to these living units, which may or may not have definite cell walls. In plants, however, as we have seen, they have walls in the vast majority of cases, and the term cell is still applied not only to the living cytoplast-nucleus unit, but also to the wall and cavity after the protoplasm has disappeared.

The adult living plant cell is generally characterised not only by the possession of a cell wall, but also by a large central vacuole or space filled with a watery liquid (cell sap), enclosed within the cytoplasm (Fig. 6, C). So large is this in proportion to the whole space within the cell that the cytoplasm is often a mere thin layer lining the wall. The nucleus, which always remains surrounded by cytoplasm, is embedded in this film and bulges it out into the vacuole (Fig. 6, C). Vacuoles are by no means the monopoly of plant cells-they frequently exist in those of animals-but animal cells as a class are not characterised by the possession of a large central vacuole, the formation of which depends on the mode of nutrition and growth of the typical plant cell. The layer of cytoplasm next the vacuole "vacuole wall"), like the external layer (ectoplasm ${ }^{2}$ ) next the cell wall, is an exceedingly thin film-a gel membrane, such as we saw is usually formed at the boundary of a colloid sol (Fig. 7).

Embryonic (Meristematic) Cells.-In the higher plants

\footnotetext{
3 Greek $\pi \rho \tilde{\omega} \tau$ os, first, and $\pi \lambda \alpha \dot{\alpha} \mu \alpha$, thing formed or moulded, as from clay or wax.

a Ėkós, outside.
} 
new cells are mainly produced by division from preexisting cells at and near the tips of the branches of the root and shoot. These regions of active cell division are called meristems. ${ }^{\mathrm{I}}$ The cells in this region are all young because they have all recently arisen from cell division. The characteristic features of such a meristematic or embryonic cell (Fig. 6, A) are that the cell wall is relatively thin, the cell cavity is completely filled with protoplasm and the nucleus is large, its diameter commonly being as much as two-thirds to threequarters that of the whole cell. The granular endoplasm ${ }^{2}$

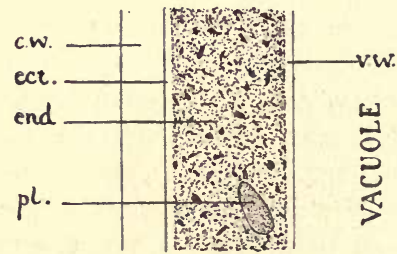

FIG. 7.-Enlarged diagram of the portion $\mathrm{X}$ of Fig. 6. C : c.w., cell wall ; ect., ectoplasm; endo, endoplasm; v.w., vacuole wall; $p l$., plastid. Supposed to be multiplied 6,600 times.

contains various granules and droplets (metabolites) of different sizes. It also contains small refringent bodies called plastids, which may under certain circumstances form starch, and if exposed to light develop chlorophyll and become chloroplasts. The endoplasm is bounded on the outside by the very thin layer of ectoplasm, which is, however, only just visible under the highest powers of the microscope and with the best optical definition, since it is only a fraction of I $\mu$ in thickness (Fig. 7).

The large and conspicuous nucleus (Fig. 6) is a

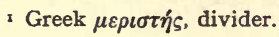
2 $z p \delta o v$, within. 
spherical body bounded by the nuclear membrane and containing a network or detached granules of chromatin bathed by a liquid, the nuclear sap. The nuclear membrane and chromatin are doubtless of gel structure, the nuclear sap a sol. There is also present in most cases a conspicuous spherical deeply staining body (sometimes more than one) called the nucleolus. This seems to be of the nature of reserve material which is used to "feed" the chromatin when division occurs. The nucleus in this condition is often called the "resting nucleus," because it is not dividing, but the "term is rather misleading, for the nucleus is really carrying out its main function, that of "directing" the nutrition and growth of the cell.

\section{Division of the Nucleus (Karyokinesis) and of the} Cell.-Meristematic cells constantly divide, and between two successive divisions there is a period of growth (increase in bulk) of the protoplasm till each daughter cell attains about the size of the mother cell before division, when each divides again. The division of the cell is always preceded by division of the nucleus, which takes place in a remarkable, complicated fashion, the process being known as karyokinesis ${ }^{1}$ or mitosis ${ }^{2}$ (Fig. 7, G). Except in the nuclear divisions preceding the formation of the reproductive cells, this process of karyokinesis shows substantially the same features in every nuclear division preceding cell division in practically all animals and plants, the differences met with being of quite minor importance.

The chromatin granules (or chromatin network [Fig. 8, a]) of the " resting nucleus " become arranged in

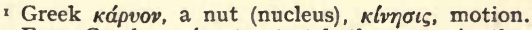

2 From Greek $\mu \iota \tau \delta \omega$, to stretch the warp in the loom, because of the thread structures appearing in the process. 
bent chromatin rods of equal length, called chromosomes (Fig. 8, b), the nucleolus and nuclear membrane $\mathbf{x}$ disappearing. A spindle-shaped structure, consisting of exceedingly delicate protoplasmic threads, appears in the cell, frequently stretching across almost its entire width (Fig. 8, c). This spindle is called the achromatic spindle, because its threads do not take up stains and become deeply coloured as do the chromosomes. The threads converge on two spots (poles). In some plant cells and in most animal cells each pole is occupied by a staining granule called a centrosome to which the threads are attached. The chromosomes now become arranged on the spindle at its equator. Each chromosome commonly has the form of a rod bent in the middle rather like a hairpin, the free ends projecting outwards, perpendicular to the long axis of the spindle, the bend directed towards the centre of the spindle (Fig. 8, d). At this time each chromosome is seen to have a double structure, the bent rod being split longitudinally, but this splitting may occur earlier, so that the double structure is visible as soon as the chromosomes appear from the resting nucleus (Fig. 8, c). Spindle fibres running from the poles towards the equator (and distinct from the fibres on which the chromosomes rest, which run continuously from pole to pole) now become attached to the chromosomes at or near the inwardly directed bend (Fig. 8, A). These fibres contract and thus pull the longitudinal halves of the chromosomes (daughter chromosomes) apart, the daughter chromosomes remaining in contact longest at the free ends (Fig. 8, $e$ and B). By continued contraction of the

In some animal cells the nuclear membrane remains intact during karyokinesis, the whole of the processes described taking place inside it. 
achromatic threads the daughter chromosomes are completely separated and pulled along the guiding

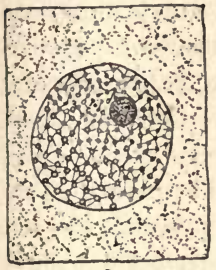

a.
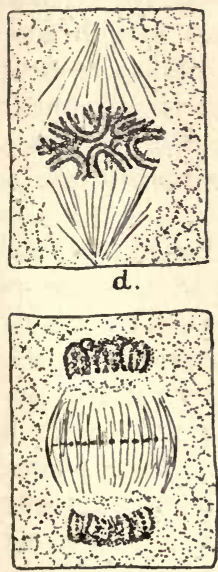

g.

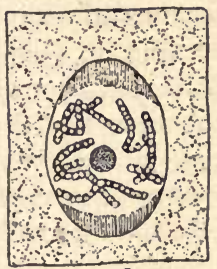

b.
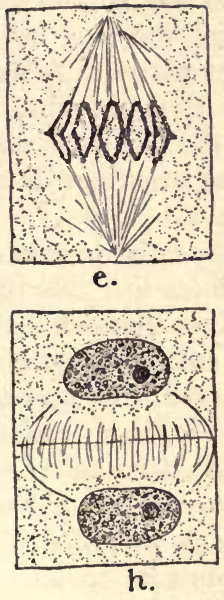

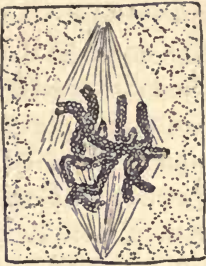

c.
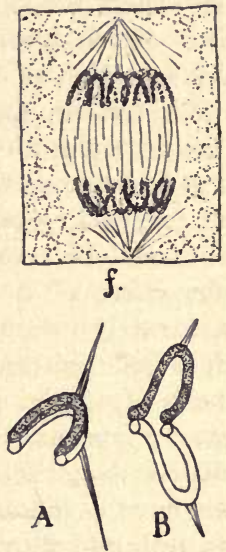

FIG. 8.-Karyokinesis in a meristematic cell (see text). Modified from Strasburger.

spindle threads (Fig. $8, f$ ) to the neighbourhood of the poles, where the chromosomes lose their separateness and individuality (Fig. 8, g), each group becomes surrounded 
by a nuclear membrane, and two daughter nuclei are thus constituted (Fig. 8, h).

At the same time (in the tissue cells of plants), thickenings of the achromatic spindle fibres appear in the equatorial plane (Fig. 8, g), i.e. midway between the two daughter nuclei, and grow till they fuse laterally, forming a plate or membrane across the cell (Fig. $8, h$ ), which becomes joined to the lateral cell walls. This membrane then becomes converted into cell wall substance, forming a new cell wall, which divides the original cell into two. The achromatic spindle then disappears, all except the equatorial portion on which the thickenings which initiated the new cell wall were formed. This portion of the threads, penetrating the new cell wall, remain, and thus maintain continuity between the protoplasm of the two sister cells. This continuity can be demonstrated as existing between the protoplasm of adult living cells.

It will be seen that the process of karyokinesis has the effect of dividing the chromatin of the nucleus accurately into halves between the two daughter cells. It is believed that each chromosome carries the organic basis of certain characteristics of the cells of the particular species of organism, and that when each is divided longitudinally into halves, these characteristics are equally shared by the two halves (daughter chromosomes), and thus each daughter cell comes to have the same characteristics as the mother cell.

Development of the Adult Cell from the Embryonic (Meristematic) Cell (cf. Fig. 6).--So long as cells remain meristematic they do not cease to pass through the rhythm of growth and division, but the products of cell division on the side towards the body of the root or shoot gradually pass out of the meristematic 
state and assume the characters of permanent tissue cells. The power of cell division does not necessarily cease, but divisions become less frequent and in some cases do not recur.

The permanent tissue cells commonly attain a size far greater than that of the meristematic cells-many of them grow especially in the direction of the axis of the root or stem, and become several times longer than they are broad. This great increase in the size of the cell does not result, however, from increase in bulk of the protoplasm, but from the formation and increase in size of vacuoles. Drops of liquid (cell sap) appear in the endoplasm, increase in size (Fig. 6, B), and eventually run together, forming one large vacuole, in the midst of which the nucleus, always surrounded by a layer of cytoplasm, is suspended by strands of cytoplasm (bridles), which run across the vacuole to the layer lining the cell wall. When the vacuole increases still further in size the bridles are thinned out till they collapse, and the whole of the cytoplasm then forms a layer on the cell wall, the centre of the cell being completely occupied by the large vacuole (Fig. 6, C, left-hand bottom cell).

During the process just described the appearance and increase in size of the vacuoles distends the extensible cell wall, which would be thinned out to the point of rupture were it not that new cellulose is continually added to it by the protoplasm lining its inner surface. When the cell has reached its definitive size this process of addition of cellulose often continues, and now the cell wall is actually thickened, the successive layers added to its inner surface being often clearly visible on each side of the original thin wall (middle lamella). This thickening is not, however, 
always quite continuous over the whole surface. At certain spots the wall may not be thickened, so that channels or pits are left, leading from the middle lamella to the cavity of the cell. The pits of adjacent cells are practically always formed opposite to one another. This arrangement greatly facilitates the passage of water and solutes from cell to cell.

Osmotic Pressure and Turgor.-What is the force which causes the distension of the cell during its growth from the embryonic to the adult condition? It is the pressure (often called "osmotic pressure") caused by the "attraction" of osmotic substances within the cell for water, which is drawn into the cell in the effort to establish equilibrium (cf. p. 57), and develops a liquid pressure against the wall. We must suppose that the vacuoles are initiated in the protoplasm by the local concentration of an osmotic substance, e.g. sugar, in certain spots, perhaps within plastids. ${ }^{\mathbf{I}}$ Water is attracted to these spots and more water enters the cell from without to replace what is thus removed from the general cytoplasm. As the pressure in the vacuoles increases the cytoplasm is pressed against the cell wall, which is itself progressively distended, just as the inner tube of an inflated bicycle tyre is pressed against the outer cover. If the cell were freely suspended in water this process would continue till the wall burst or till the elastic reaction of the wall counterbalanced the distending force due to the attraction of the osmotic substance for water. But when the cell is surrounded by other cells similarly distended they all press against one another and give rigidity to the whole tissue. This condition of a tissue is called turgor or turgidity,

I In any case a semi-permeable membrane is formed round each vacuole, whether derived from a plastid or formed in the endoplasm. 
and herbaceous plants depend upon it for maintaining their stiffness and the erect position of their shoots.

Wilting and Plasmolysis.-If a plant cell loses water faster than it can obtain it, turgidity cannot be maintained, the cell becomes soft and limp, and the tissue loses its rigidity. This is what happens on a very hot day when plants lose water by evaporation to the air faster than they can replenish their supply by root absorption. The plant is said to wilt. The loss of turgidity takes place even more rapidly when a plant is pulled up by the roots and left in the sun.

Turgidity can also be lost in another way. If the cell or tissue be placed in a sugar or salt solution of osmotic strength greater than that of the cell sap, water is drawn out of the cell against the pull of the cell sap. The protoplasm is then no longer held tight against the wall by the pressure from within, and it contracts away from the wall as the liquid pressure within diminishes by the withdrawal of water. This process is called plasmolysis. The osmotic substances of the cell sap do not, however, pass out through the semi-permeable membranes represented by the vacuole wall and ectoplasm, and if the cell or tissue be placed again in water this re-enters the cell, whose turgidity is re-established.

\section{PRACTICAL WORK.}

(I) Examine the growth of the bean roots set up in the corked bottle last time. They show (a) local growth, (b) curvature from the horizontal to the vertical plane, i.e. the bending of an organ in response to an external force. Sketch diagrammatically these results alongside the sketches of the roots as originally set up.

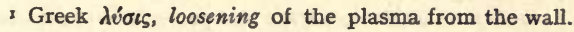




\section{The Plant Cell.}

(2) Strip off the surface layer of cells from the piece of onion scale provided. Examine and draw carefully under the high power two or three of the cells, marking the parts.

(3) I Tease out in a drop of water a little of the pulp of the Snowberry, taking care that it is kept well wetted. Note that the tissue is loose, with many air spaces between the cells, many of which are spherical. Examine the cells under the high power. Note that the proportion of cell sap to protoplasm in each cell is very high. Look for the nucleus. Staining with dilute iodine solution will often help in case of difficulty.

(4) Examine the stained longitudinal section of the tip of a bean root. Note that the actual tip is formed by the " root-cap." The area just behind this is the "growing point" or primary meristem, and consists of small mostly square cells densely filled with protoplasm and with large conspicuous densely staining nuclei. Draw carefully under the high power $(a)$ examples of meristematic cells with " resting nuclei," $(b)$ different stages of nuclear division " (compare with demonstration slides), (c) different stages of vacuolation of the cells behind the meristem.

Measure the diameter of a meristematic cell and of its nucleus, also the length of one of the longest cells in the section.

\section{Cells of Animal Tissues}

(5) Examine the demonstration specimens of stained and mounted animal tissues. 3 Note cytoplasm, nucleus and absence of cell wall: also, e.g. in cartilage, the intercellular substance or " matrix."

\section{Turgidity and Plasmolysis.}

(6) Place one of the turgid bean roots (a) in ro per cent. $\mathrm{CaCl}_{2}$ solution, 4 leaving the other $(b)$ on the table. After a few minutes note that $(a)$ has become quite limp. Place it in water, and it gradually regains its turgidity; $(b)$ gradually wilts as it loses water by evaporation to the air.

I This may be omitted if the Practical Work is too long.

- Demonstration slides under immersion objectives showing stages in karyokinesis should be available if possible.

3 Three or four slides will suffice: columnar epithelium, cartilage, connective tissue and a preparation of muscle showing nuclei are suggested as suitable.

$4 \mathrm{NaCl}$ solution is liable to injure the protoplasmic membranes. 
(7) With a knife split a length of fresh bean stem longitudinally into quarters. Note that the ends of the strips curve outwards. The harder tissues of the outside of the stem arè in a state of elastic tension in the intact stem, i.e. they are tending to contract in the longitudinal direction, but cannot do so against the swelling force of the turgid cells of the centre (pith) of the stem, which are similarly prevented from actually swelling. When the stem is divided longitudinally the inner cells swell and the outer ones contract, so that each strip of stem bends outwards.

(8) Strip off a fragment of the coloured surface layer of Tradescantia leaf as free as possible from the green tissue below. Mount in water and examine under the high power. The vacuoles of the purple cells are filled with coloured cell sap. Place a drop of Io per cent. $\mathrm{CaCl}_{2}$ solution on the slide just touching the edge of the coverslip, and draw the solution under the coverslip by holding a fragment of blotting paper against the opposite edge. Watch the effect on the cells-plasmolysis. Reverse the action by running in a drop of water in the same way and watch the recovery of turgor. 


\section{THE GREEN PLANT CELL}

Chloroplasts and Chlorophyll. - The majority of plants we are familiar with have green stems and leaves, or green leaves alone. The green colour is due to the mixed pigment chlorophyll present in the chloroplasts (which are definite, oval, spherical or disc shaped, protoplasmic bodies lying in the cytoplasm of certain of the living cells of the leaf or stem), and in these alone. The lower green plants (for instance the Mosses and Liverworts) possess chloroplasts of the same nature as those of the seed plants, but in the Green Algæ the chloroplasts are very various in shape and size (e.g. spiral bands, Fig. 9). The chloroplasts are derived from the plastids (which multiply by simple division) found in the meristematic cells, and are not formed afresh in the protoplasm.

Chlorophyll is a mixture of four different pigments held in the protoplasm of the chloroplast in a colloid state: chlorophyll a $\left(\mathrm{C}_{55} \mathrm{H}_{72} \mathrm{O}_{5} \mathrm{~N}_{4} \mathrm{Mg}\right)$ giving a bluegreen solution, chlorophyll $\beta\left(\mathrm{C}_{55} \mathrm{H}_{70} \mathrm{O}_{6} \mathrm{~N}_{4} \mathrm{Mg}\right)$ giving a pure green solution, carotin ${ }^{\mathrm{I}}\left(\mathrm{C}_{40} \mathrm{H}_{56}\right)$ giving orange crystals, and xanthophyll $\left(\mathrm{C}_{40} \mathrm{H}_{56} \mathrm{O}_{2}\right)$ giving yellow crystals. These are mixed in the chloroplasts in the proportion of six molecules of the two green pigments to two of the two orange and yellow ones.

r Carotin also occurs in other parts of various plants. It is, for instance, the red colouring matter of carrots, of many red and orange coloured flowers, etc.; also of the "eyespot" in Chlamydomonas (see p. 186). 
Photosynthesis. - The spectrum of chlorophyll shows definite absorption bands. ${ }^{\text {I }}$ These absorption bands represent the rays most strongly absorbed by the chlorophyll, and thus the radiant energy available for the work which the chloroplast carries on. That these are the rays actually used in photosynthesis, or at least in one or more of the processes that go to make up photosynthesis, can be shown by a very pretty experiment first devised by the German botanist Engelmann. A thread of green alga living and carrying on its work in water is illuminated by a solar spectrum so that different parts of the thread are illuminated by different coloured rays. A culture of a certain

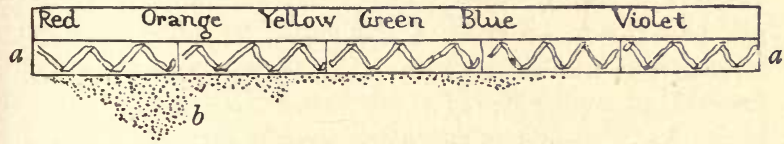

FIG. 9.-Diagram illustrating Engelmann's experiment, to show by the aggregation of a motile bacterium (b) highly sensitive to oxygen the regions of maximum evolution of oxygen by a green alga $(a-a)$ illuminated by a solar spectrum. The most active rays correspond with the red and orange.

bacterium (Bacterium termo) which is very sensitive to oxygen, so that its cells move towards any source

I When a beam of white light is split up by passing through a prism into a band or spectrum of differently coloured rays, owing to the different angles through which the different rays are bent by the prism, and is allowed to fall on a white surface, the spectrum so formed (solar spectrum) is continuous. But if the beam has passed through a translucent coloured substance, the spectrum formed shows dark bands (absorption bands) corresponding inversely with the colour of the translucent substance, the colour of the substance being caused by the combined effect on the eye of the coloured rays that have passed through it. The absorption bands are caused by the absence of the rays that have been stopped or absorbed by the coloured substance. Thus a pure red substance shows absorption in the other colours of the spectrum-blue, green, etc., a blue substance in the red, orange, etc., and so on. 
of oxygen diffusing into the water, is then introduced in the neighbourhood of the illuminated algal thread. The minute cells of the bacteria swarm towards and cluster round those portions of the thread illuminated by the coloured rays corresponding with the chief absorption band of chlorophyll, especially in the red (Fig. 9).

This behaviour of the bacteria shows that free oxygen (one of the products of the process of photosynthesis) is being given off most actively at those points; and consequently that those particular rays are the ones most active in photosynthesis.

Sugar, probably glucose, is the first product of the process of photosynthesis that can be definitely recognised in the green cells of the leaf, free oxygen being liberated at the same time. These substances are only formed in the presence of a certain intensity of the proper rays of the spectrum (pure white sunlight contains all the rays), and in the presence of carbon dioxide and water. We may represent the general equation of photosynthesis thus :-

$$
6 \mathrm{CO}_{2}+6 \mathrm{H}_{2} \mathrm{O}=\mathrm{C}_{6} \mathrm{H}_{12} \mathrm{O}_{6}+6 \mathrm{O}_{2}
$$

It is likely, however, that formaldehyde is first formed :-

$$
\mathrm{CO}_{2}+\mathrm{H}_{2} \mathrm{O}=\mathrm{CH}_{2} \mathrm{O}+\mathrm{O}_{2}
$$

and that the formaldehyde is then condensed to form glucose :-

$$
6 \mathrm{CH}_{2} \mathrm{O}=\mathrm{C}_{6} \mathrm{H}_{12} \mathrm{O}_{6}
$$

but this is uncertain, since formaldehyde has not been demonstrated in the leaf. Nevertheless the "reduction " of $\mathrm{CO}_{2}$ in presence of water to form formaldehyde and also the condensation of formaldehyde to form glucose are both operations that can be performed in 
the chemical laboratory. For the first process considerable energy is required, and it is this that certainly requires, in the chloroplast, the radiant energy of light. This first process may then be designated as photolysis, the second process, that of condensation to form sugar being a process of chemosynthesis. For ordinary biological purposes, however, we may consider them together as photosynthesis.

Structure and Functions of the Green Plant Cell.The green tissue (mesophyll $\mathrm{I}$ ) which forms the bulk of an ordinary foliage leaf (see Fig. Io) consists of cells with thin cellulose walls lined inside by a layer of cytoplasm enclosing the nucleus and numerous chloroplasts, and with a large central vacuole. These cells are, in fact, " adult " living cells (see p. Ior) containing chloroplasts (Fig. II).

The mesophyll tissue is covered by a layer of colourless living cells (epidermis) on the surface of the leaf, and the outer walls of these cells, in contact with the external air, has a waterproof layer of cell wall substance (cuticle) which prevents the cells of the leaf drying up by evaporation to the air (Fig. Io, st.) The epidermis (and cuticle) is, however, pierced by a number of holes (stomata ${ }^{2}$ ) which lead from the external air to the air-containing intercellular spaces between the mesophyll cells. These intercellular spaces are intercommunicating, and are so arranged that every mesophyll cell has some part of the outer surface of its wall in contact with the internal atmosphere of the leaf.

The internal atmosphere of the leaf, being in communication with the external air through the stomata, has at first the same composition as the latter, but this composition is continually being modified by

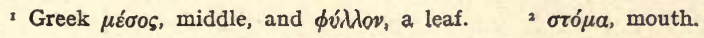


the interchange of gases between it and the mesophyll cells, and equilibrium between it and the external air is constantly tending to be re-established by the diffusion of gases through the stomata, into or out of the leaf. Thus if the mesophyll cells absorb carbon dioxide, as they do when illuminated, the pressure of this gas decreases in the intercellular spaces and fresh carbon dioxide from the air streams in through the

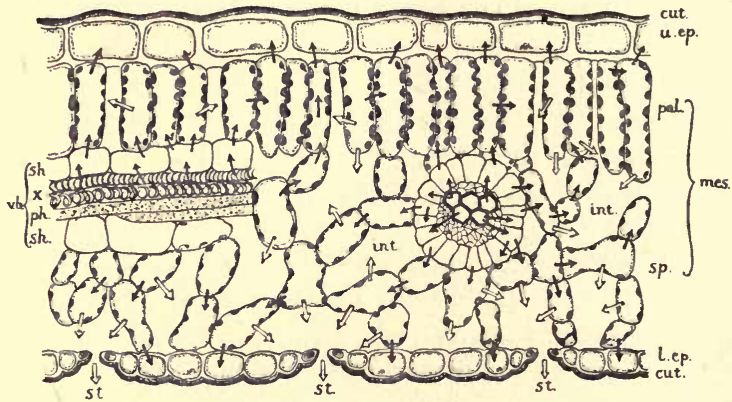

FIG. I0.-Diagram of transverse section of a leaf to show the relation of the mesophyll (mes.) to the system of intercellular spaces (int.) and the stomata (st.). The white arrows represent the evaporation of water from the mesophyll cells into the intercellular spaces, and its diffusion through the stomata to the outer air.

stomata to restore the equilibrium. The oxygen produced in the mesophyll cells under the same conditions increases the pressure of oxygen in the intercellular spaces, and it escapes through the stomata into the external air. The air in the intercellular spaces is normally nearly saturated with water vapour, owing to the constant evaporation of water from the wet mesophyll cells into the air in contact with them, and this water vapour is always escaping through the 
stomata if the outer air is drier, as it usually is, than the air inside the leaf (Fig. Io, white arrows).

Let us consider now what happens in the chloroplast of a mesophyll cell which is suddenly well illuminated. The light falling upon it is robbed of those rays of the spectruin corresponding with the absorption bands
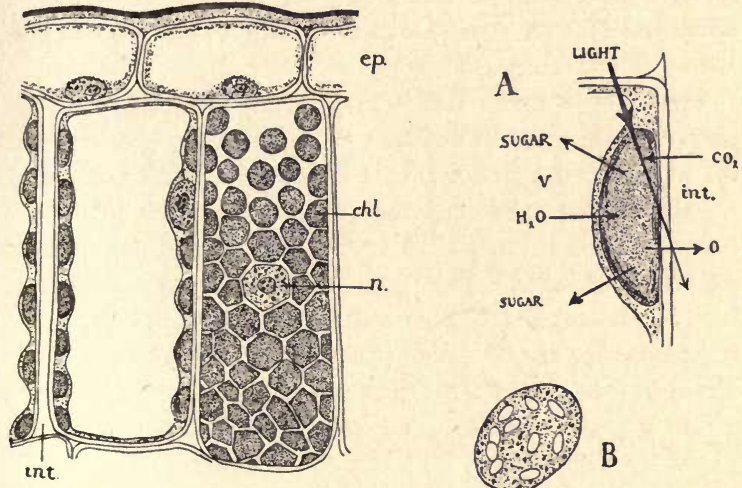

FIG. I I.-Two mesophyll (palisade) cells. Frg. 12.-A, diagram of chloThe one on the left is seen in optical section, the one on the right in surface view. Above are two cells of the upper epidermis. chl., chloroplast; $n$, nucleus; $c . w$. , cell wall; ep., epidermal cell; int., intercellular space.

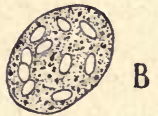
roplast of mesophyll cell to show its activity when illuminated; $v$, vacuole ; int. intercellular space B, starch grains formed in chloroplast.

of chlorophyll. The energy so obtained is used to split up the molecules of carbon dioxide provided by the respiration of the protoplasm, and the carbon is united with the elements of water (always present in the cell) with the ultimate formation of sugar, oxygen at the same time being liberated (Fig. I2, A). Some of the sugar and oxygen so formed is doubtless used 
in the respiration of the colourless protoplasm, but, under favourable conditions, the rate of photosynthesis far exceeds the rate of respiration in a green cell. This means in the first place that far more carbon dioxide is used than can be obtained from the process of respiration, the pressure of the gas in the cell decreases and fresh supplies enter from the air of the intercellular spaces. This in turn causes an inflow of carbon dioxide through the stomata.

The oxygen formed as a by-product of photosynthesis increases the pressure of this gas in the cell, and is given off at the cell surface into the intercellular spaces and thence diffuses through the stomata into the open air, so that well illuminated leaves are constantly giving off free oxygen. The sugar continuously produced by the chloroplasts diffuses into the cell sap. Some of it passes into the bundles (veins) of the leaf and along the bundles to other parts of the plant. But more sugar is produced than can be removed from the mesophyll by this means, and the concentration of sugar in the cell continuously rises during the day.

Formation of Starch in the Chloroplasts.-In most green plants, though by no means in all, the excess of sugar after a certain degree of concentration is reached, is converted into starch by condensation, and this forms small grains in the substance of the chloroplast :-

$$
n \mathrm{C}_{6} \mathrm{H}_{12} \mathrm{O}_{6}=\left(\mathrm{C}_{6} \mathrm{H}_{10} \mathrm{O}_{5}\right)_{n}+n \mathrm{H}_{2} \mathrm{O}
$$

A foliage leaf of a starch-forning plant at the close of a bright day when large amounts of sugar have been formed shows its chloroplasts packed with starch grains (Fig. I2, B), as can easily be seen by examining a section of the leaf under the high power. The starch grains appear as bright granules in the substance of 
the chloroplast. The mass of starch formed in the leaf, and the dependence of this process upon illumination, can be strikingly demonstrated to the naked eye by covering part of the leaf during the day with tinfoil while still on the plant and leaving the rest exposed. If in the afternoon the leaf is cut off, killed with boiling water, the colour taken out with alcohol, and the leaf is then placed in a dish and flooded with iodine solution, the part of the leaf that was exposed to light will quickly turn blue-black owing to the staining of the starch grains as the iodine gradually penetrates the tissues, while that part of the leaf protected from light remains colourless.

The function of the chloroplasts as starch formers is quite distinct from their function as sugar producers. It does not depend directly on the photosynthesis of sugars or on the green colour of the chloroplasts, but on the actual concentration of sugar in the leaf. The leaves of many plants do not form starch at all, and the concentration of sugar in the leaf necessary to lead to starch formation varies considerably in different species. The power of forming starch from sugar is shared by the colourless plastids (leucoplasts) found in the colourless cells of the plant, particularly in seeds, tubers, etc., but also in stems and roots, where large quantities of starch are formed from the sugar which comes to these cells from the leaves. Chloroplasts themselves can form starch from sugar which they have not made, as can be proved by the experiment of floating detached living leaves on sugar solution in the dark, when starch will appear in the chloroplasts.

As the illumination decreases the rate of sugar formation likewise decreases, and when it falls below a certain intensity - in natural illumination about sunset- 
the photosynthesis of sugar becomes negligibly small, Since a certain amount of sugar is always being removed from the cell through the veins, the concentration of sugar in the mesophyll cells falls, and the starch grains in the chloroplasts are gradually reconverted into sugar during the night, and this sugar is likewise conveyed away by the veins. Thus both during the day and during the night there is a constant stream of sugar away from the leaf to other parts of the plant where it is used in respiration, in the formation of new protoplasm and new cell walls, or is stored as starch, or sometimes remains as sugar (beetroot, sweet fruits, etc.), or as some other carbohydrate such as inulin (Jerusalem artichoke, etc.), or is converted into fats.

The formation of glucose from starch and of starch from glucose may be represented by the general reversible equation :-

$$
n \mathrm{C}_{6} \mathrm{H}_{12} \mathrm{O}_{6} \underset{\text { hydrolysis }}{\stackrel{\text { condensation }}{\rightleftarrows}}\left(\mathrm{C}_{6} \mathrm{H}_{10} \mathrm{O}_{5}\right)_{n}+n \mathrm{H}_{2} \mathrm{O}
$$

The reaction is catalysed by the enzymes diastase and maltase, probably in either direction (see p. 45 for the intermediate substances formed).

Photosysthesis in a Green Cell living in Water.-In a green cell living in water the processes of sugar and starch formation are essentially the same as in the mesophyll cell of a leaf. The only difference is that the carbon dioxide is derived directly from that which is dissolved in the water instead of being absorbed from the air, and that the sugar does not leave the cell. Starch (or in some species fat) is formed when the concentration of sugar passes a certain point. The sugar is used for respiration, to form the cellulose for cell wall substance, and also (together with the elements of 
mineral salts entering the cell from the surrounding water), to make the proteins which form the basis of the new protoplasm produced as the cell grows and divides.

Respiration and Photosynthesis.-It will be noted that the general equation representing respiration is the exact reverse of that representing photosynthesis. The two equations may be combined thus :-

\section{RESPIRATION}

potentiai energy $\longrightarrow$ liberation of energy $\longrightarrow$ kinetic energy

$$
\mathrm{C}_{6} \mathrm{H}_{12} \mathrm{O}_{6}+\underset{\text { (reduction) }}{\stackrel{\text { (oxidation) }}{\rightleftarrows}} 6 \mathrm{CO}_{2}+6 \mathrm{H}_{2} \mathrm{O}
$$

potential energy $\longleftarrow$ locking up of energy $\longleftarrow$ kinetic energy PHOTOSYNTHESIS

In the mesophyll cell of a well-lighted leaf the photosynthetic process very greatly exceeds respiration, so that carbon dioxide and water are continuously used up, sugar and free oxygen produced. When illumination falls to a certain low level of intensity the two processes will exactly balance, and the system represented by the green cell will be in equilibrium in respect of these processes. In still weaker light or in the dark photosynthesis stops altogether and sugar and oxygen are continuouly used up, carbon dioxide and water produced. Thus it is true that green plants give off carbon dioxide in the dark. But the popular notion that it is " unhealthy " to keep plants in a bedroom at night is nevertheless unfounded-the amount of the gas given off as a result of the respiration of the plant cells is far too small to raise appreciably the carbon dioxide content of the air.

Synthesis of Proteins.-The formation of sugars in the green cells, their condensation into starch, and the 
hydrolysis of this to sugars again, form a very important part of the metabolism of green plants. Sugar is not only used by the plant in respiration, but it is also the material from which the cell walls, the skeleton of the plant, are formed. For these reasons the carbohydrate metabolism of the plant makes up much the greater part of the whole of its metabolic processes. But the synthesis of proteins to form the basis of the new protoplasm which is constantly being formed in meristematic cells is clearly also of essential importance. This appears to take place by the interaction of soluble carbohydrates with the nitrogen and sulphur derived from the nitrates and sulphates absorbed by the roots. It seems that comparatively simple organic nitrogenous substances called amino-acids are thus formed, and that these are synthesised by successive condensations (analogous to the condensations of sugar to starch or cellulose) into more complex nitrogenous substances, and eventually into proteins. These anabolic processes probably occur in the mesophyll cells of the leaf, whence the nitrogenous substances are conveyed, in relatively simple form, to growing organs and storage organs. At least the final synthesis of the protoplasmic proteins must also take place in the meristematic cells where new protoplasm is actually being formed. It is not possible to enter here into the details of these processes, some of which are still largely obscure and others unknown. Some of the simpler proteins-the polypeptides - can be made synthetically in the laboratory.

The Raw Materials of Plant Food.-The chemical elements which are constituents of the molecules of the most important organic substances involved in the structure of organisms have been enumerated on page 37 . Of these the green plant obtains carbon from the carbon 
dioxide of the air, hydrogen and oxygen largely from water, nitrogen, sulphur and phosphorus from nitrates, sulphates and phosphates dissolved in the water of the soil. The remaining elements essential to the nutrition of plants are potassium, magnesium, calcium and iron, and these are obtained from the same mineral salts. Other elements, such for instance as sodium, silicon, etc., are habitually absorbed by the plant, but they are not essential as materials of food. This can be proved by growing the plant not in soil but in a mixed solution of various salts. Such water cultures, as they are called, show that a plant can grow and flourish when it is provided with a selection of soluble salts in dilute watery solution containing only the elements named. A good "complete" water culture solution is the following :-

\begin{tabular}{|c|c|c|}
\hline Calcium nitrate & & \\
\hline Potassium nitrate & • & $\mathbf{I}$ \\
\hline Magnesium sulphate & & . \\
\hline Potassium phosphate & • & .. I gra \\
\hline Distilled water & . & . 50 \\
\hline
\end{tabular}

This is a stock solution. 2 or 3 c.c. should be used for I litre of culture solution, distilled water being added to make up the litre, and a drop of iron chloride added. Other soluble salts can be substituted provided they contain the same elements, for instance potassium nitrate, calcium phosphate, calcium sulphate, magnesium chloride.

But if one of the elements represented in the salts is omitted from the solution the plant does not flourish, and eventually dies. The most rapid failure results from the omission of nitrogen, and this is clearly because nitrogen is an essential constituent of the protein molecules. When the store of combined nitrogen in the seed has been used up by the growing plant, no 


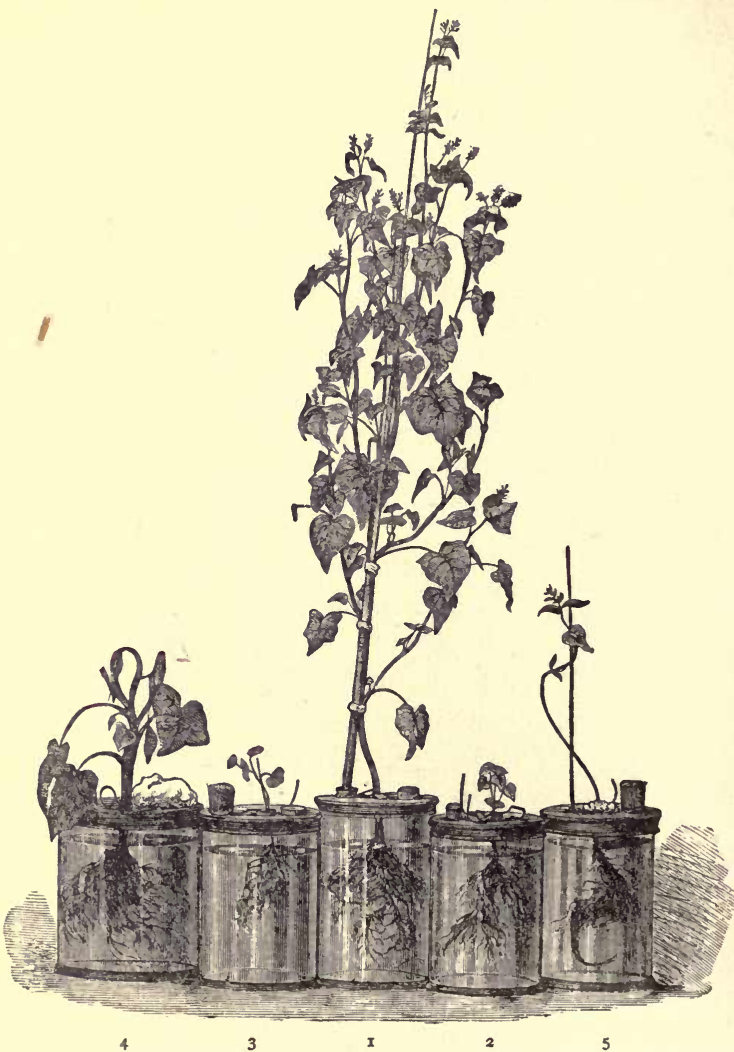

Fig. I3.-Water cultures of Buckwheat (after Nobbe). I, plant grown in complete solution; 2, without potassium; 3, with sodium instead of potassium; 4, without calcium; 5, without nitrogen. 
new protoplasm can be formed, and growth necessarily ceases unless fresh supplies of combined nitrogen in a suitable form, i.e. as nitrate, can be obtained. The ordinary green plant cannot directly use the free gaseous nitrogen of the air to build up its proteins. And the plant cannot develop successfully, and dies sooner or later, in the absence of any one of the other essential elements.

\section{PRACTICAL WORK.}

(I) Soak the leaf provided in alcohol and note that a green pigment is gradually extracted from the leaf. After an hour's soaking pour some of the green solution into a test tube and examine with the hand spectroscope. Compare with (6).

(2) Strip off part of the colourless surface layer of cells (epidermis) of the leaf of Gladiolus provided. Below you will see colourless ribs (veins) with strips of green tissue (mesophyll) between. Cut out a small portion of this green tissue with the point of a sharp scalpel or penknife and mount in a drop of water, taking care to wet the tissue thoroughly. Put on a coverslip and examine under the low power. Note the small cells with green dots, and the air spaces (now partly filled with water) between them. Examine with the high power and carefully draw one of the cells of which you can get a clear view, marking the cell wall, the cytoplasm lining the wall with chloroplasts embedded in it, the central vacuole, and the small shining spherical nucleus. Stain a portion of the green tissue with hæmatoxylin for ten minutes and note that the nucleus especially absorbs and becomes coloured by the stain.

(3) Examine under the microscope in a drop of water a whole leaf of the water weed Elodea. This is a "water leaf," wholly submerged in nature and of very simple structure, consisting, except for the veins, of only two layers of cells, all containing chloroplasts. The structure of each cell is essentially the same as that of the mesophyll cell of Gladiolus, though the cells are much larger and oblong in shape. The nucleus is generally difficult to detect : it is often hidden in a clump of chloroplasts. When visible it is seen as a rather large, pale, often faintly granular oval body. Note that all the chloroplasts are embedded in cytoplasm : none of them lie in the large central vacuole. The cytoplasm may sometimes be seen streaming actively round the cells, carrying the chloroplasts with it. (Cf. p. 85.) 
(4) Mount a cross section of the fresh stem of Pellionia in a drop of water. Notice under the high power the chloroplasts in the cells near the surface of the section, with starch grains (bright colourless granules) inside them. In cells further from the surface note that the starch grains are larger and have burst out of the chloroplasts. The chloroplast which has formed the grain can often be seen attached to one end of the grain. The growth of these deeper lying grains is continuous and is probably the result of a continuous supply of sugar, while the sugar supply of the superficial cells probably depends, as in a mesophyll cell, directly upon photosynthesis and therefore upon light, so that the starch grain ceases to grow and is converted back into sugar every night. Draw examples under the high power of different relations of starch grain and chloroplast, and then test with iodine.

(5) With the hand spectroscope compare the spectrum of the alcoholic solution of chlorophyll (I) with that of the translucent green leaf and with the spectrum of sky light. [Compare also with the demonstration spectra.] Note the absorption bands of the chlorophyll spectrum.

(6) Examine the demonstration water cultures, and observe the effects of leaving out each of the essential elements in the mixed solution of salts used to feed the plant. 


\section{THE COLOURLESS PLANT CELL. THE YEAST PLANT}

WE have already seen that many of the living cells of the higher green plants do not contain chlorophyll, e.g. the meristematic cells, all the cells of the root, many of those in the interior of the stem, and also those which form the surface layer (epidermis) of herbaceous stems and leaves. The cytoplasm of many of these cells, however, contain plastids, which may turn green on exposure to bright light, and thus become chloroplasts. All these colourless cells have to be fed with sugar, ultimately coming from the green cells, to repair the waste of respiration; and the meristematic cells, which are actively dividing, not only require large quantities of sugar, but must also be supplied with nitrogenous substances to make new protoplasm.

There are also plants which have no green cells: a few saprophytes and parasites ${ }^{I}$ among the seed plants which live upon the organic substances of humus (decaying vegetable substance such as leaf mould) or upon the organic substances of the bodies of living green plants; the great group of FUNGI; and finally the group of unicellular plants known as BACTERIA, which, as we shall see in the next chapter, are of extraordinary importance in the economy of the life of the 
world. All of these colourless plants, with the exception of a few kinds of bacteria, obtain the materials for the formation of new protoplasm and the energy for carrying out their life processes by absorbing liquid organic substances from outside.

Yeast (Saccharomyces ${ }^{\mathrm{I}}$ ). - A simple organism with which it is convenient to begin the study of colourless plants is the unicellular yeast plant. There are many different kinds or species of yeast, some of them differing markedly in the appearance and size of the cell, but mainly distinguished by the difference of their activities. There is convincing evidence that the yeasts are derived from a certain group of the higher fungi, but they live and maintain themselves indefinitely as unicellular plants.

Structure (Fig. I4).-The single yeast cell is spherical or oval in shape, about 8 to I $2 \mu$ in diameter-a small cell on the scale of the tissue cells of the higher plants, but about the same size as a cell of Protococcus-surrounded by a cellulose cell wall. The cytoplasm is more or less granular, and there is a central oval vacuole, though the diameter of this in proportion to that of the whole cell is not so great as in the ordinary adult tissue cell of the higher plant-in other words, the cytoplasmic layer lining the cell wall is relatively thick (Fig. I4, C). The central vacuole is really intranuclear-fine threads of chromatin applied to the inside of nuclear membrane extending around the vacuole from an aggregation of chromatin at one end (Fig. I4 C, chr.). This aggregation of chromatin can sometimes be seen as a granule in the living cell. According to the metabolic condition of the cell, other small vacuoles or droplets of liquid, and granules of × "Sugar-fungus." 
various substances, may appear in the surrounding cytoplasm (Fig. I4, C).

Conditions of Life.- "Wild" yeasts are found in
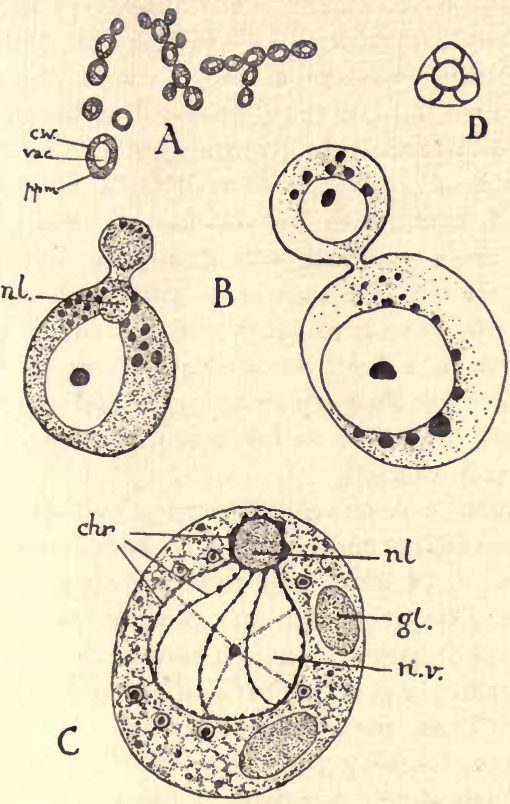

Fig. I4,-Yeast plant (Saccharomyces). A, yeast cells budding and forming chains. $\times 200$. c.w., cell wall; ppm., protoplasm; vac., vacuole. $B$, two cells forming buds. The black granules are volutin, a complex organic substance formed by the yeast cell. C, diagram of the structure of a yeast cell made up from information about its structure obtained from treating and staining the cell with various reagents; $n . v$., nuclear vacuole; $\boldsymbol{n l}$., nucleolus; chr., chromatin; $g l$., glycogen (after Wager and Penistone). $D$, formation of four spores within a cell.

exposed sugary secretions or exudations of plants, for instance in the "nectar" of flowers, in the drops 
of juice appearing on the surface of a fruit with ruptured skin, and the like. But the yeasts, like the wheat plant or the apple tree, are best known in the "domesticated" or cultivated condition. There are two main uses to which yeast is put by man-the fermentation of sugary plant juices to make alcoholic drinks, such as beer, wine, or cider, and the " raising" of bread by means of the carbon dioxide given off by active yeast.

"Brewers' yeast" is a frothy, viscous, creamcoloured liquid with an "alcoholic" smell. "Bakers' yeast" is a similarly coloured putty-like substance. A little of either placed in a drop of water under the microscope is seen to consist of myriads of yeast cells, but while in bakers' yeast these are mostly single, in brewers' yeast they are united in chains which are often branched, the result of rapid reproduction by budding (Fig. I4, A and B).

Nutrition.-Yeast can live in a culture solution of salts containing the essential elements of the food of protoplasm, of which the most complex is ammonium tartrate $\left(\mathrm{NH}_{4}\right)_{2} \mathrm{C}_{4} \mathrm{H}_{4} \mathrm{O}_{6}$, an organic salt containing carbon, hydrogen and oxygen as well as nitrogen. The other elements can be obtained from simple inorganic salts such as potassium phosphate, $\mathrm{K}_{3} \mathrm{PO}_{4}$, calcium phosphate, $\mathrm{Ca}_{3}\left(\mathrm{PO}_{4}\right)_{2}$, and magnesium sulphate, $\mathrm{MgSO}_{4}$. With these alone, therefore, it can construct proteins. But it grows very slowly in such a medium, for the amount of energy it can obtain from ammonium tartrate is small. If sugar be added to the solution it grows and multiplies much faster, for from the sugar it can obtain a large amount of energy.

Reproduction.-Yeast reproduces itself primarily by budding (Fig. I4, A and B). A tiny area of the cell wall, usually at one end of the oval cell, is thrust out by the 
osmotic pressure within the cell, cytoplasm passing into the bud. The bud grows until it reaches the size of the mother cell, the nucleus of which meanwhile divides, one of the daughter nuclei passing into the new cell. The daughter cell may then be cut off and become a new unicellular yeast plant. So actively, however, does budding take place in a solution containing sugar (with the other necessary elements present in a suitable form) that the daughter cell produces another bud before separation has taken place, and this again another, and thus the chains of yeast cells are formed. A cell may even bud in two or three places at once, so that the chains are branched.

Spore Formation.-Another method of reproduction may occur, however, if the yeast is starved, e.g. by being left on a slab of plaster of Paris with a little water. Under these circumstances the nucleus divides into two and then into four, the cytoplasm withdraws from the wall and becomes aggregated round the four neclei in four tiny spherical masses, each of which secretes a comparatively thick wall (Fig. I4, D). Each of the spores so formed is about $3 \mu$ in diameter. If now the yeast culture dries up, the original cell wall collapses, and the spores can be carried away and float with the dust in the air, the living contents remaining in a dormant condition for a long time. Falling into a suitable liquid medium, the dormant protoplasm of the spore absorbs the liquid and becomes active, bursting the spore wall, pushing out, and covering itself with a new ordinary cell wall, thus giving rise to a new vegetative yeast cell.

The spores of yeast are therefore a resting stage in the life history, able to withstand desiccation for some time. This is true of most of the spores formed by plants. 
Alcoholic Fermentation.-The yeast plant is of special interest both biologically and practically, because it is able for a time, in a suitable nutritive medium containing sugar, to carry on a process which we must regard as a modified kind of respiration, in place of ordinary respiration. The yeast cells produce an enzyme (zymase), by the catalytic activity of which the molecule of sugar (glucose) is split into two molecules of ethyl alcohol and two of carbon dioxide :-

$$
\underset{\text { glucose }}{\mathrm{C}_{6} \mathrm{H}_{12} \mathrm{O}_{6}}=\underset{\text { ethyl alcohol }}{2 \mathrm{C}_{2} \mathrm{H}_{6} \mathrm{O}}+\underset{\begin{array}{l}
\text { carbon } \\
\text { dioxide }
\end{array}}{2 \mathrm{CO}_{2}}
$$

thus liberating energy from the sugar molecule without using free oxygen. This process in the living cell is called anaerobic respiration, as opposed to the ordinary aerobic I respiration, in which free oxygen is used. Of the sugar actually absorbed by the yeast cells some is split up in this way and the energy is used in growth, while some is doubtless used, together with nitrogenous substances, for the formation of new protoplasm. None of the zymase formed by the cell passes out into the surrounding sugar solution, but quantities of sugar pass into the yeast cells, and are split up into alcohol and carbon dioxide. The carbon dioxide forms bubbles in the liquid, which froths, while the alcohol accumulates, and the energy set free raises the temperature of the liquid. Meantime the yeast cells grow and bud with great rapidity, enormously increasing their number. This is the process known as alcoholic fermentation, and is the foundation of the making of beer and wine.

Brewing. - The liquid fermented in brewing beer is called wort, and is a hot water infusion of malt, i.e. sprout-

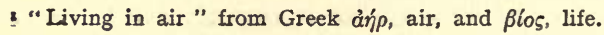


ing barley grains. Water is added to the fresh barley grains and kept standing on them about three days, being changed two or three times during that period. The wet grains are then spread out on the floor of the malting house, and occasionally raked over so as to allow the air free access to them. The temperature of the grains rises considerably during the week or so they are left on the floor. This is due to the production of heat by the respiration of the grains, which absorb oxygen from the air. The starch stored in the grains is converted into sugar, and much of this sugar is split up in respiration. At the end of the " malting," however, the sprouting grains are still full of sugar and of soluble nitrogenous organic substances-the reserve stores of the seed are mobilised for the growth of the young plant.

The malt is then removed to kilns and dried for one or two days at $70^{\circ}$ or $100^{\circ} \mathrm{C}$., according to the kind of beer that is to be produced..$^{\text {The kilned malt is }}$ removed to vats to which water is added at $50^{\circ} \mathrm{C}$., the temperature being afterwards raised to $76^{\circ} \mathrm{C}$. This is the process of infusion of the malt, the liquid produced being the wort, which is now filtered off, the malt residue, which still contains a considerable amount of nutritive substance, being used as cattle food. The wort is boiled with hops for two or three hours to extract the bitter aromatic substances from the hops, and then sieved off and run into underground marble vats, where it is cooled to $8^{\circ} \mathrm{C}$., and the yeast-a pure culture of a particular strain of yeast ${ }^{2}$-is added. Here it stands

I The details of malting and brewing procedure given are those followed at the famous Carlsberg Brewery at Copenhagen, where "Pilsner" and "Lager" light beers are the products. The malt is kilned at $70^{\circ} \mathrm{C}$. for the former and at $100^{\circ} \mathrm{C}$. for the latter.

"In the case of "Pilsner" and "Lager" beer a so-called " bottom yeast," which sinks to the bottom of the wort. For brewing the heavier English beers "top yeasts"" are used. 
for twelve to fourteen days while the process of fermentation takes place. The carbon dioxide produced rises to the top, so that the surface of the fermenting wort becomes covered with a thick layer of froth. Much of the carbon dioxide escapes, but being heavier than air lies on the surface of the vat, so that workmen have sometimes been asphyxiated by approaching the vats incautiously. The temperature of the wort rises, owing to the great liberation of energy in fermentation, but is kept down to $\mathrm{I} 2^{\circ} \mathrm{C}$. by currents of cold air directed on to the vats. When the fermentation has reached the desired point the beer is run into specially lined storage casks or tanks and kept at $\mathrm{I}^{\circ} \mathrm{C}$, for three months, when it is ready for bottling.

The whole of the installation is kept scrupulously clean, or infection of the wort with " wild "yeasts and bacteria would occur, undesired fermentations would take place, and the beer spoiled.

The kind and quality of beer produced depends not only on the kind of yeast used and the time during which fermentation proceeds, but also on the kind and quality of the malt and hops (and these again on the strains of barley and hops, and on the soil and climate in which they are grown), for beer is a complex solution of many substances derived from different bodies contained in the malt and hops, besides the sugar, which is the main substance fermented.

Wine-Making.-In wine-making the fermentable liquid (must) is grape juice, which is pressed from the overripe grapes in a wine-press. The yeasts which cause the fermentation in the must are always present on the overripe grapes, and do not have to be added as is done in the making of beer. As in the case of beer, but to a far higher degree, the kind and quality 
of wine depends not only on the way it is made, but on the kind of grape used, the climate and soil in which the vines are grown, and the weather during the season in which the grapes ripen. Thus not only are claret, port, sherry and hock, for instance, totally different wines, because they are made in different ways, but particular vineyards in each wine-growing district produce particular qualities of wine, and finally the different years or " vintages" from the same vineyard have different characteristics owing to the weather during the year. Sometimes the characters so produced actually override the differences due to the different vineyards, so that a connoisseur can recognise a particular vintage. A fine wine is an immensely complex solution, and the chemistry of many of the organic substances it contains is still very imperfectly understood. Slow chemical changes continue in the wine while it is in cask and while it is in bottle. Up to a certain point the wine constantly "improves," and after that point deteriorates.

In making sparkling wines the later stages of fermentation are allowed to take place in the bottles, and the carbon dioxide, which develops a considerable pressure, is thus retained. Fermentation is, however, always stopped before it is complete and the yeast removed.

\section{Relation of Fermentation to Respiration. - The} alcoholic fermentation of sugar by the yeast plant, or rather by the enzyme zymase which it produces, is, as has been said, to be regarded as a modified kind of respiration into which free oxygen does not enter (anaerobic respiration). But yeast cannot go on fermenting sugar indefinitely. After a time it must have free oxygen and respire aerobically. It is interesting to note that ordinary (aerobic) respiration begins with 
a splitting of the sugar molecule without oxidation, free oxygen only taking part in the reaction towards the end of the process, resulting in the formation of carbon dioxide and water. Alcohol may actually be formed in ordinary plant cells if free oxygen is excluded. Thus alcoholic fermentation by yeast represents the first stage of the ordinary respiratory process continued for a long time and very energetically.

Alcoholic fermentation of sugar by yeast is only one kind of fermentation. Sugar can be split up into other substances than ethyl alcohol and carbon dioxide, and a great variety of other organic substances can be split up in a similar way. These varied fermentations are mainly carried out by various members of the great class of unicellular plants, the Bacteria, with which we shall deal in the next chapter.

\section{PRACTICAL WORK.}

\section{A. Yeast.}

(I) The corked bottle contains yeast fermenting a sugar solution. Dip a glass rod into lime-water and test the air in the bottle for carbon dioxide in the same way as with the respiring barley (p. 89). Repeat the test at the end of the practical work.

(2) Place a drop of brewers' yeast on a slide, cover, and examine with the high power. Measure the length and breadth of several of the isolated cells and determine their average size. Draw two or three on a large scale, marking cell wall, cytoplasm and central (nuclear) vacuole. A refractive mass (chromatin) can often be seen at one end of the vacuole. Note also the granules and small vacuoles that can often be seen in the substance of the cytoplasm. Stain with Schulze's solution. The cell wall stains blue (cellulose).

(3) Trace the method of budding by which the yeast cells multiply and draw examples of different stages.

(4) Smear a drop of yeast culture thinly over a perfectly clean coverslip and dry the smear over the flame of a lighted match. Place five drops of methylene blue in a clean watchglass and fill it up with water. Immerse the coverslip in the 
stain and leave it there for one minute. Take it out and wash by gently moving the coverslip in water. Dry both sides of the coverslip with blotting paper. Find out on which side the smear is, and place the coverslip, smear downwards, on a small drop of dilute glycerine on a slide. Examine with the high power.

\section{B. Thick Cellulose and Mucilaginous Cell Walls.}

(I) Examine in water sections of the date-stone (seed of the date-palm) cut so as to show the elongated cells in transverse section. Note the very thick cell walls. These are penetrated by pits running from each cell cavity and ending at the middle lamella of the cell wall (the middle lamella was the original thin wall separating two adjacent cells before thickening took place). The pits of adjacent cells are always exactly opposite one another.

Mount the section in a drop of Schulze's solution, and leave for ten minutes. Then note that the mass of the cell wall is stained purple (cellulose), while the middle lamella remains unstained or only faintly stained. The mass of cellulose is converted into sugar when the seed germinates and the sugar is used for the respiration and growth of the young plant.

(2) Examine a dry seed of the flax plant (linseed). Note the sharp outline of the opaque seed. Now put the seed in a watchglass with a little water. The outline of the seed is soon surrounded by a transparent fringe. Compare the feel of the dry and the wet seed between the fingers.

Now examine a thin section of the seed $d r y$ under a coverslip, and, running in a drop of water, watch the swelling of the surface layer of cells. The middle lamellæ of the walls separating the cells can be distinguished from the swollen mucilaginous mass of the walls. Note also the cuticle bounding the walls on the outside. Methylene blue will stain the mucilage and render it more easily visible. 


\section{CHA PTER IX}

\section{BACTERIA}

BACTERIA are the smallest of known organisms, but they are of quite extraordinary importance in the economy of nature, an importance only exceeded by the green plants. While the latter form the basis of all life by building up living substance from inorganic materials, the former destroy dead organic matter, breaking it down into simpler forms and eventually into inorganic substances which are available for the food of green plants. Bacteria are also of direct practical importance to men, not only because some of them cause various deadly diseases, but because others are used by him, as yeast is used, to carry out fermentations -not alcoholic fermentations, but processes of the same general nature, such as the "ripening" of cheese, the "curing" of tobacco, and so on. There are a large number of different species of bacteria known, and certainly far more exist than have yet been recognised.

Size, Shape and Structure.-The diameter of an average bacterial cell is only about I $\mu$, far less than that of other unicellular organisms. There certainly exist, however, living organisms which may probably belong to the Bacteria and which are too small to recognise under the highest powers of the microscope. I Thus we know that yellow fever, and also swine fever, are

I It will be remembered that an object less than about ${ }^{5} \mu$ in diameter cannot be clearly defined under the highest powers of the microscope (p. 50). 
caused by different specific living organisms which are invisible and which will pass through the meshes of a porcelain filter that excludes any visible bacterium. It is at least probable that many different kinds of such ultramicroscopic organisms exist, for there is room between the lower limit of microscopic visibility and the size of a complex protein molecule for a collection of such molecules to be associated into a system which would form an ultramicroscopic protoplasmic unit with specific characters.

The visible bacteria may be classed in two groupsa group of simpler forms which are the more numerous, and a more highly developed group with which we need not concern ourselves here. The simpler forms may be roughly classed according to their shape into three types: (I) the coccus, which is spherical, on the average I $\mu$ in diameter; (2) the bacillus, a straight cylindrical rod, I $\mu$ or less in diameter and up to about Io $\mu$ long; and (3) the spirillum, a curved rod which may be spirally coiled like a corkscrew. Some bacteria, however, are not of these shapes, but may be oval in outline, etc. (see Fig. I5). When bacteria were first beginning to be systematically studied, sixty or seventy years ago, these "form types" were largely used as generic names, the different species being distinguished according to the habitat or activity, and some of these species are still recognised, e.g. Bacillus coli (which lives in the human intestine), Bacillus anthracis (which causes the disease anthrax), and so on. It is not, however, the form of the bacterial cell but its specific activity that is its most important feature, and many new genera have been established.

Each bacterial cell consists of a minute mass of protoplasm in which a separate nucleus cannot be 
detected. The whole of the protoplasm stains strongly with the ordinary nuclear stains, and it is likely that what corresponds with the chromatin of the ordinary
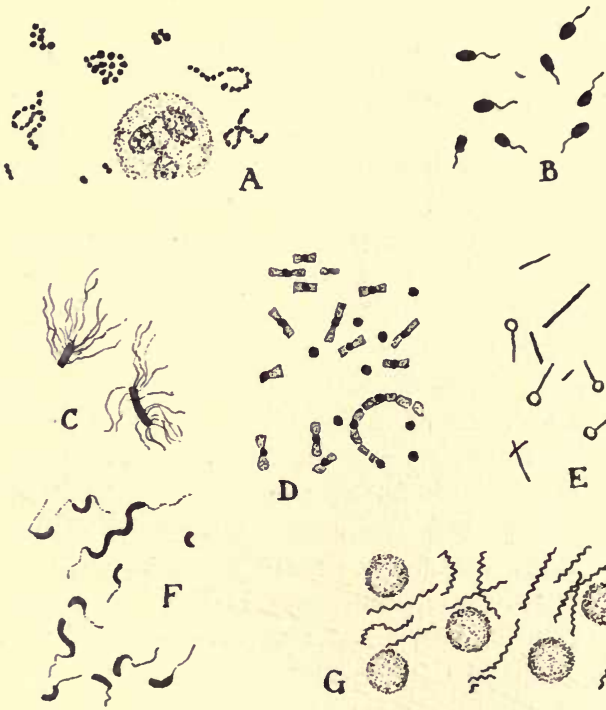

D
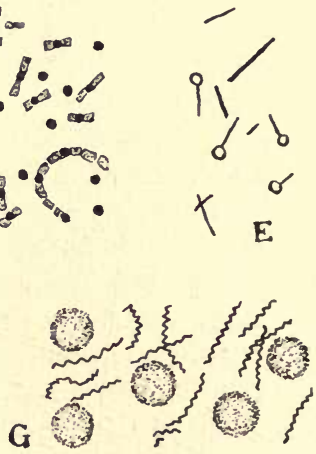

Fig. 15.-Various forms of Bacteria. $\times 1,000$. A, Staphylococci (groups) and Streptococci (curved chains) from pus (the large spherical body is a pus corpuscle). B, Nitrosomonas, a nitrifying bacterium from soil, with single flagellum. C, Bacillus typhosus (typhoid), showing flagella. D. Bacillus anthracis (anthrax), showing spores (one in the centre of each cell). E, Bacillus tetani (tetanus). Note the spherical spore at one end of and much broader than the cell. F, Cholera spirillum, with a flagellum at each end of the cell. G, Spirochate pallida (syphilis spirillum). After Muir and Ritchie.

cell nucleus exists in the bacterial cell equally distributed through the cytoplasm. The protoplasm is surrounded by a membrane of gel structure (as is always 
the case on the surface of a protoplasmic unit). This is not composed of cellulose, and is not to be compared with the cell wall of an ordinary plant cell. In some kinds of bacteria the colloid membrane swells very greatly in the liquid medium inhabited by the organism, and thus the individual cell, or group or colony of cells, may become surrounded by a thick mucilaginous investment, making the whole colony quite visible to the naked eye and slimy to the touch. A colony of this kind is called a zooglcea.

Flagella or cilia (extremely delicate threads of protoplasm arising from the surface of the cell, singly or in groups, and projecting into the surrounding medium) may be detected in many species by special methods of staining (Fig. I5, B, C, F).

Movement and Response to Stimuli.-Many kinds of bacteria swim about actively in a liquid medium, in most cases no doubt by the beating of their flagella, for these have never been detected in the non-motile forms. Some bacteria, however, especially the spirilla, appear to move by wriggling their bodies (rather as an eel moves), i.e. by the contractility of the cell protoplasm as a whole.

Motile bacteria move, as a rule, in response to chemical stimuli, i.e. towards some substances and away from others (positive and negative chemotaxis). A good example is Bacterium termo, mentioned on p. II 3 as specially sensitive to free oxygen and used in Engelmann's experiment to pick out the particular rays of the spectrum in which the chloroplasts liberate most oxygen.

Nutrition.-The foods of bacteria are very various both in kind ant? in chemical nature, and their habitats naturally correspond with these different foods. Some 
species feed directly on the proteins of living animals or plants, some on various solid or liquid organic substances in the living body or outside it. Some can get their nitrogen from comparatively simple organic salts like ammonium tartrate, just as yeast can ; others get it from even simpler salts ; others, again, can use the free nitrogen of the air. A few can form their protoplasm from inorganic substances alone, as green plants can, though they do it in quite a different way, using the energy liberated in the oxidation process instead of the energy of sunlight. The great majority, however, feed on more or less complex organic substances, from which they obtain energy for growth and multiplication.

The actual form in which the food is absorbed by the bacterial cell must of course be liquid or gaseous -in the great majority of cases it is liquid. That is why we class bacteria as plants, and, so far as their nutrition is concerned, with the great group of colourless plants known as the fungi. But both in structure and affinities (by which we mean descent) the bacteria are very different from and most probably have no connexion with the fungi proper. Bacteria are most probably more or less directly derived from the most primitive forms of life, of which we have no direct knowledge. We must, however, conceive of primitive organisms as consisting of very minute forms living in water, and obtaining their energy in different waysfrom light, from chemical energy such as that liberated in the oxidation process, or from ingesting other minute organisms. From this undifferentiated group the green plants developed along one line ; the animals, with their habit of feeding on other organisms, along another; while the bacteria as we know them to-day are a highly 
specialised group, most of which feed on liquid organic substance, while retaining an exceedingly simple structure. Bacteria in the same forms shown by existing species have been found preserved in fossils many millions of years old.

Reproduction.-The simpler bacteria multiply by simple division of the cell and separation of the daughter cells so formed. Sometimes the cell grows to a larger size than the normal before division; in other cases the cell divides and each daughter cell subsequently grows to the normal size. The daughter cells of a bacillus frequently remain together for a time after division, so that lines of cells having the form of jointed rods are produced.

In most bacteria growth and multiplication proceed with great rapidity. Under very favourable conditions a bacterium may reach maturity and divide in twenty to thirty minutes. If division takes place only once an hour, seventeen million individuals will have arisen from a single cell in twenty-four hours. The chief factors which control the rate of multiplication are temperature and food supply.

Spore Formation.-In certain species of the simpler bacteria, chiefly bacilli, spores are produced under certain conditions. These spores are formed in the same sort of way as yeast spores, by the aggregation of the protoplasm at a given spot in the cell, the protoplasm of the spore becoming surrounded by a dense wall. As a rule only one spore is formed in each cell, either in the centre or close to one end. When the spore comes into conditions suitable for growth, the spore wall is split, and a new vegetative cell protrudes, assuming the form characteristic of the species.

Bacterial spores are by far the most resistant form 
of living protoplasm known. Many can withstand for some time exposure to dry heat of considerably over $100^{\circ} \mathrm{C}$, while most vegetative forms are quickly killed at $50^{\circ} \mathrm{C}$. In some cases spores can even withstand immersion in boiling water for several minutes, while they may remain alive in a dry and inactive condition for years. The great difference between the powers of resistance of vegetative cells and spores is illustrated by the anthrax bacillus (B. anthracis). The active vegetative cell is killed by two minutes' exposure to I per cent. carbolic acid, while spores resist immersion in the same strength in some cases for fifteen days. Vegetative forms can, however, withstand extreme cold for a long time, for instance the temperature of liquid air $\left(-190^{\circ} \mathrm{C}\right.$. $)$ for twelve months.

Dispersal.-Spore formation is not a means of multiplication in bacteria any more than it is in the case of yeast. Only one spore is normally formed in the bacterial cell, and the growth and division of the organism is completely arrested until the spore germinates. The spore condition is the resting stage of the bacterium, enabling it to withstand conditions unfavourable for vegetative growth and multiplication. Spores are, of course, an important means of dispersal for the forms which produce them, since they are passively carried about in currents of air without injury, whereas vegetative bacterial cells will sooner or later be killed by drying up. But only comparatively few species of bacteria are known to produce spores, most kinds being dispersed entirely by means of the ordinary cells. Bacteria often get into the air by detachment of minute particles from the surface on which they are growing. Such particles form part of the dust of the air, and prac- 
tically all air at low levels, except over mid-ocean, contains more or less dust. Dust is carried about in currents of air, and is often blown by the wind for very long distances. When the air becomes comparatively quiet, the particles drift slowly down and settle. Any living cells which it contains, vegetative bacterial cells, bacterial spores or fungal spores, will grow if they find themselves in suitable conditions of moisture, food supply and temperature. In such conditions spores will germinate, and such vegetative cells as have not been killed by drying up will grow and divide.

Habitats and Conditions of Life.-This is why all dead bodies exposed to air putrefy and eventually fall to pieces, provided they remain moist and not too cold ; and why all milk, beer or wine similarly exposed turns sour. These processes all depend on the activity of the living protoplasm of bacteria. Each is carried out by particular species only, and cells or spores of some of these are always found in the dust of the air, so that they are certain to fall sooner or later on to the surface of the dead body or into the organic liquid. These processes go on more rapidly at fairly high temperatures, simply because such temperatures are the most favourable for bacterial growth and metabolism; and they are suspended at $0^{\circ} \mathrm{C}$. or below (though the bacteria are not killed), because protoplasm cannot work at the freezing-point of water.

In order to feed, live and multiply, each species of bacterium must have the appropriate conditions for that species, the right sort of food, the presence (or absence) of oxygen, a sufficiency of water in the medium, and a suitable temperature. The temperature relations of bacteria are remarkably wide and various. Most species live at the ordinary temperatures which prevail 
in the part of the world where they are. In temperate climates few species will grow at temperatures above $30^{\circ} \mathrm{C}$., though some occur in manure heaps, etc., whose optimum ${ }^{\mathrm{I}}$ is as much as $70^{\circ} \mathrm{C}$. Species which are parasitic on warm-blooded animals live best at $37^{\circ} \mathrm{C}$., and some of them die at temperatures less than $20^{\circ} \mathrm{C}$.

Sterilisation.-If all bacteria and their spores are to be destroyed in a given enclosed space, the air in that space may be raised to a temperature which they cannot survive. This can usually be effected by dry heating to $160^{\circ} \mathrm{C}$. for half an hour or to $180^{\circ} \mathrm{C}$. for ten minutes. If a liquid is to be similarly " sterilised," it may be boiled in a flask for several minutes, with a cotton wool plug inserted in the neck of the flask. The contents of the flask will then be thoroughly cleared of active cells. The plug acts as a filter to prevent the entrance of cells or spores during cooling when air is drawn into the flask through contraction of the cooling air inside. Spores in the liquid may, however, survive the boiling. Heating with steam at high pressure (which raises the temperature of the steam) at $\operatorname{II}^{\circ}{ }^{\circ} \mathrm{C}$. for ten minutes, or $120^{\circ}$ for five minutes, will kill anything, even the most resistant spores.

A flask containing a fermentable or putrescible liquid, i.e. a liquid in which bacteria can grow and bring about changes in the chemical composition, thus plugged and sterilised, can be kept for any length of time without change. If the plug is removed at any time fermentation will at once begin and the characteristic bacteria will be found in the liquid. This is a clinching proof, originally due to the great French chemist and biologist Pasteur, that all such changes are due to the activity of micro-organisms.

Most favourable temperature. 
An alternative method of sterilising a liquid which avoids the use of heat, and has also the advantage of excluding the bodies of bacteria, is by filtering through unglazed earthenware. The liquid to be sterilised is forced under pressure through the filter, whose pores must be so small that they will not allow the smallest bacterium to pass. To be efficient such a filter must be made of the finest " china clay." But in practice sterilisation by filtration is uncertain, because of inequalities in the size of the pores and imperfections in the filter. Such filters are often attached to domestic water taps to make sure that drinking water contains no harmful bacteria.

Pure Cultures.-In the early days of the study of bacteria it was impossible for the observer to be sure that he was not dealing with a mixture of different kinds. The cells in a living culture of bacteria might show various forms, or bring about various effects on the culture medium from time to time. This might be due to the powers of a single kind of bacterium, or on the other hand to the gradual disappearance of one species and the multiplication of another (which was also present) as the chemical constitution of the medium was altered owing to the effect of the bacteria upon it. No increase of accurate knowledge of the different kinds of bacteria was possible under these conditions.

The discovery of the possibility of complete sterilisation of a medium by Pasteur not only proved that all changes of the nature of putrefaction and fermentation were brought about by living organisms, but it enabled perfectly pure cultures of a single kind of bacterium to be made and kept. The making of pure cultures for the study of bacteria was introduced about the year 
I 880 by the great German bacteriologist, Koch, and has been an indispensable means of developing the vast modern science of bacteriology. The various technical methods of making pure cultures are based on the fact that if a small quantity of a liquid containing bacteria is diluted with a large excess of sterile water and the bacteria evenly distributed through it, each drop of the mixture will contain only a few or even a single organism. Such a drop added to a sterile culture medium will probably give rise to a growth consisting of one sort of bacillus only. In practice it is more convenient to immobilise the bacteria in the diluted mixture by making it with a warm gelatine or agar jelly, which will set when it is cold. The individuals then multiply and give rise to visible "colonies," each originating from one organism, which can be transferred to other sterile media, and the forms of the cells and the effects of their growth on the medium can be studied at leisure.

Genera, Species and "Strains" of Bacteria.-The original "genera" of bacteria, based on form names such as bacillus (rod), coccus (sphere), spirillum (spirally curved rod), are still to some extent retained, but have been considerably added to as knowledge has increased, and the present classification is partly based on form and partly on activity. Thus rod forms are still called Bacterium and Bacillus, the genera of cocci are distinguished by prefixes (Micrococcus, Streptococcuscells arranged in chains, Staphylococcus-cells arranged like bunches of grapes, etc.). Many of the simpler spiral forms are placed in Vibrio and Spirillum, while the long spiral forms which move by contractility of the body protoplasm and have no flagella are placed in Spirochate. Other genera are Sarcina (a coccus 
form growing in rectangular masses), Nitrobacter (oxidising nitrates in soil), etc.

The species of bacteria are often called after the name of the discoverer, or from their form, colour, activity or habitat: in the case of the pathogenic (disease producing) forms, the species is often called after the name of the disease, e.g. Bacillus tuberculosis, the form causing phthisis and other forms of tuberculosis. Some species are so closely alike in form that they can only be distinguished by their effect upon the medium in which they are cultivated, or upon the animal in which they live. Thus Bacillus typhosus of typhoid fever resembles so closely $B$. coli communis, a regular inhabitant of the human intestine, that they can only be distinguished by the wide difference in their actions (for instance whether they can or cannot ferment various kinds of sugars), the former producing a dangerous disease through the substances it excretes.

So far as we know the species of bacteria are as constant as those of other organisms, like always producing like, but different "strains" belonging to the same species may differ rather widely in their activities; for instance one may be specially virulent as a disease producer, while another may give rise to quite mild symptoms, so that, as in the higher plants, we have what are called " aggregate species" and " elementary species" (pure strains). How far strains of widely different activities are produced solely by the effect of changed conditions, we do not certainly know. Changed conditions undoubtedly do produce great differences; thus by growing Bacillus coli in a culture medium containing a trace of carbolic acid it is easy to obtain a race which is much more resistant to the disinfectant action of the phenol than is the normal 
species. Among the enormous number of kinds of bacteria, a few have become parasitic on the higher animals and cause disease. Hence, as in the past, new diseases may make their appearance when suitable circumstances occur, by the adaptation of a previously harmless form to new conditions in an animal body, where it produces poisonous substances, giving rise to the symptoms of the disease.

Respiration and Fermentation.-Many bacteria, like the vast majority of animals and plants, can only live in the presence of free oxygen. These are called aerobic 1 forms (cf. p. I32). Some, on the other hand, like yeast, can do without free oxygen for a time, and others, again, cannot live in its presence (anaerobic forms), and obtain their energy by splitting up organic substances without oxidation.

Bacteria which live on complex organic substances, as most of them do, nearly always cause profound changes in the medium they inhabit. They break down the organic substances of the medium (presumably by the excretion of enzymes) just as yeast breaks down sugar. The term fermentation is technically applied to all such processes, and is not confined to alcoholic fermentation as in popular usage. Different kinds of bacteria ferment different substances. Thus certain bacteria found in milk (Bacterium acidi lactici and many others) ferment milk sugar (lactose), splitting one molecule of lactose (with a molecule of water) into four molecules of lactic acid :-

$$
\underset{\text { lactose }}{\mathrm{C}_{12} \mathrm{H}_{22} \mathrm{O}_{11}}+\underset{\text { water }}{\mathrm{H}_{2} \mathrm{O}}=\underset{\text { lactic acid }}{\mathrm{C}_{3} \mathrm{H}_{6} \mathrm{O}_{3}}
$$

and the accumulation of the lactic acid in the milk turns it sour. Alcohol itself is split up by certain s Living in air, from Greek $a \dot{\eta} \rho$, air, and $\beta l o s$, life. 
bacteria, especially the "vinegar plant," Bacterium aceticum, in the presence of oxygen, into acetic acid and water:-

$$
\underset{\text { ethyl alcohol }}{\mathrm{C}_{2} \mathrm{H}_{6} \mathrm{O}}+\underset{\text { acetic acid }}{\mathrm{C}_{2} \mathrm{H}_{4} \mathrm{O}_{2}}+\mathrm{H}_{2} \mathrm{O}
$$

Their activity is the cause of the souring of wine or beer if left standing in the air, and is used in the making of vinegar. The "ripening" of cheeses, the "curing" of tobacco, the "retting" of flax (the isolation of the fibres of the flax plant when the stems are placed in water), and many similar processes useful to man depend on fermentations carried out by different bacteria; and so do many others which are not so welcome, such as the turning rancid of butter and the turning bitter of sweet fruit such as a cut melon, as well as the putrefying of meat, etc.

Putrefaction and Subsequent Changes in Nitrogenous Organic Substances.-Putrefaction is the general name given to various fermentations of the highly complex proteins which form the basis of protoplasm. These fermentations result in the formation of a great number of compounds of varying degrees of complexity containing nitrogen and sulphur, among which are the evilsmelling gases so characteristic of the putrefactive processes. Putrefaction is carried out by various bacteria which are very widely distributed and many of which form spores, so that practically no dead body on the earth's surface can escape them. The process takes place in all dead substances rich in proteins which remain moist and not too hot or too cold to allow of the activity of the putrefactive bacteria. Freezing arrests their action, and so do the antiseptic substances formed in peat bogs. That is why meat is kept in cold 
storage, and the bodies of animals which have been engulfed in bogs are often found perfectly preserved after many years.

After the first putrefactive decompositions other kinds of bacteria reduce the products of putrefaction to simpler substances and so on until ammonium $\left(\mathrm{NH}_{4}\right)$ compounds are produced, together with carbon dioxide and water. This process is noticed in the strong smell of ammonia $\left(\mathrm{NH}_{3}\right)$ arising from a heap of stable manure at a certain stage of decomposition. The débris of plants-fallen leaves, twigs, etc.-undergo similar decompositions, but since a smaller proportion of their substance is formed of proteins and there is a much larger proportion of carbohydrates (cellulose, etc.), the result is more carbon dioxide and water and less ammonia than in the case of animal substances. Also the early stages of decomposition of plant remains are largely carried out by fungi, though bacteria also play a part. The decaying plant débris, together with any decaying animal bodies or other animal substances present, form the humus of the soil. Humus is always in process of reduction to the simple chemical substances named above, together with various mineral salts, and is as constantly replenished by fresh organic débris.

When the organic substances have been brought into the state of simple salts containing nitrogen, other kinds of bacteria take up the work, some (Nitrosomonas) converting the ammonium salts into nitrites, i.e. salts formed from nitrous acid $\left(\mathrm{HNO}_{2}\right)$ and bases present in the soil, others again (Nitrobacter) carrying out a further oxidation and producing nitrates, formed from bases and nitric acid $\left(\mathrm{HNO}_{3}\right)$. This process of the oxidation of ammonium compounds to nitrates, 
which takes place in two stages, each effected by a special set of bacteria, is called nitrification.

Nitrates are the form in which green plants mostly absorb their nitrogen supply through their roots, so that the whole series of bacterial activities described results in providing green plants with exactly the kind of food they require, and at the same time gradually remove dead bodies and organic débris from the surface of the earth and make room for fresh life.

During the progress of conversion of complex nitrogenous compounds into simple ones a great deal of nitrogen is lost to the air in the form of the free gas, but certain soil bacteria (e.g. Azotobacter, Clostridium) are able to fix this and incorporate it into the substance of their bodies. Other bacteria (Pseudomonas) which live in tubercles that are formed on the roots of certain plants such as those of the pea family (Leguminosa) are also able to absorb free nitrogen from the air, and the green plant in which the bacterium lives gets the benefit of this, eventually breaking up and absorbing the products of the dead bodies of these tubercle bacteria. Thus a certain amount of the nitrogen lost to the air during the process of decomposition is again brought into the form of living protoplasm in these ways.

Circulation of Nitrogen and Carbon in Nature.-In all these ways the nitrogen contained in the proteins of the protoplasm and other nitrogenous substances of plants and animals is converted during their decay into a form in which it can be used again by green plants, so that a constant circulation of nitrogen is always going on in nature, through the agency on the one hand of various kinds of bacteria which break down the complex substances into simple ones, and on the other of green plants which build them up again into 
complex ones. In this circulation the animals play a comparatively small part, for they merely convert the plant proteins into the proteins of their own bodies.

Besides the circulation of nitrogen there is also a circulation of carbon, which appears in the form of carbon dioxide, not only as the result of the respiration of plants and animals, but also during decomposition of the complex carbon compounds of animal and plant bodies, notably the carbohydrates found in the cellulose of plants, but also the fats and proteins. Distinct bacteria carry out the disintegration of the cellulose of which humus is largely composed, and this process ultimately results in the production of carbon dioxide and water (as well as hydrogen and marsh gas). The carbon dioxide is then, as we know, used by the green plant to build up its body.

Pathogenic (Disease-producing) Bacteria.-A small proportion of the total number of species are partly or wholly parasitic on plants and animals in which they cause disease. Partial parasites (such as Bacillus tetani) live naturally in soil containing organic matter and only occasionally gain entrance to the animal body. Complete parasites (such as the spirochæte of syphilis, or the coccus of cerebro-spinal fever) have no life outside the body, and their existence depends on their being passed on directly from one person to another.

Bacteria often invade living animal bodies by way of wounds, and may be a great danger to life, for instance on battlefields, owing to the poisons or toxins which they produce. The bacteria are carried into the wound either by the instrument causing the wound, or from the clothes, or by dirt afterwards getting on to the wounded surface. The most important are the species of Streptococcus and Staphylococcus; and these 
may get into the blood stream through the wound and thus be carried through the body, bringing about septicamia (blood poisoning).

The immense progress of modern surgery has been made possible, first by the use of antiseptics, i.e. by treating the wound with a substance (such as weak carbolic acid) which destroys the bacteria. The introduction of the use of these in surgery was due to Joseph Lister (afterwards Lord Lister), and resulted in a great decrease in the death-rate of surgical patients from blood poisoning. Later on aseptic methods of surgery were introduced, in which the surgeon's hands, instruments and dressings used are carefully sterilised, and the wound thus kept free from all bacteria.

Besides the Streptococci and Staphylococci, other kinds of bacteria may be introduced into wounds contaminated with soil rich in dung, and cause such specific diseases as tetanus (Bacillus tetani) and gas-gangrene (Bacillus welchii), so called because of the large amount of gas produced in the wound by the action of the bacillus on the tissues. These are both spore-producing bacilli which inhabit the intestines of horses and cows, where they do no harm. The spores persist for a long time in the manured soil, and germinate when they enter the wound, producing active cells which form dangerous toxins that often lead to death.

In the case of most "infectious" diseases, human beings directly or indirectly infect one another with spores or active cells of various pathogenic species, and these give rise in the body to different specific toxins which cause the symptoms of the corresponding disease. Some of the most serious among them are tuberculosis in its various forms (Bacillus tuberculosis), syphilis (Spirochate pallida), typhoid or enteric fever 
(Bacillus typhosus), epidemic meningitis or "spotted fever" (Meningococcus), plague, cholera, diphtheria, influenza, etc.

\section{PRACTICAL WORK.}

BACTERIA.

Note that all bacteria are extremely small, and very careful focussing with the high power is necessary to find them.

(I) Bacteria in Potato Extract.-Mount a drop of the turbid liquid from the surface of water in which a slice of potato has been soaking, put on a coverslip and observe the numerous rod-shaped aerobic bacteria (Bacillus mesentericus) in motion; also from the deeper layer of water the rod shaped cells, tapering at the ends of the anaerobic Clostridium butyricum. Note the different staining of the two with iodine.

(2) Note the elongated form and the rapid spiral movements (like those of eels) of the Spirilla from horse dung which has been kept in water for a week. Kill with iodine solution and again observe.

(3) Bacillus subtilis (hay bacillus).-(a) Mount a drop of hot water infusion of hay, and observe the delicate rod-shaped bacilli in motion. (b) Examine a preparation showing the same species fixed and stained. Compare their size with that of a yeast cell by means of the micrometer eyepiece.

(4) Stain a drop of each of the bacterial cultures (2) and (3a) by the coverslip method used for yeast (p. I36 (4)).

(5) Examine the sections of a nodule of the root of lupin (or other leguminous plant), and note the "bacteroids" (dead bacteria) in the central tissue.

(6) Remove the cover from the dish of sterilised gelatine or agar jelly provided, thus allowing the dust from the air to settle on it. Replace the cover after twenty minutes, and label the dish with your name. The dishes should be examined again after a week's interval.

Demonstration Specimens.--Examine carefully the demonstration slides, tubes and plates of various bacteria.

[A few representative types of culture and stained preparations of various pathogenic bacteria should be obtained for exhibition. They can usually be borrowed from a pathological laboratory. Cultures and slides of Bacillus tetani and B. anthracis, showing spores of B. tuberculosis, and of Staphylococcus and Streptococcus are suggested as suitable.]

The student should be warned not to handle the cultures. 


\section{CHAPTER $\mathrm{X}$ \\ SAPROPHYTIC FUNGI}

\section{Mucor ANd Penicillium.}

THE Fungi differ from the Bacteria in being plants whose bodies are composed, not of single cells and cell colonies, but of delicate branching threads which grow in liquid or solid "substrata" containing organic substances which they can absorb as food, and often sending branches into the air. This system of branching threads is called the mycelium, and the individual branches are called hypha. The mycelium of many species is large and conspicuous. As a general rule fungi can only grow actively in damp situations, and they are commonest when and where the air contains a large amount of water vapour.

Fungi may be either saprophytic or parasitic (see p. 78), i.e. they may either take their food exclusively from the tissues of a living organism (plant or animal) or they may take it exclusively from a non-living organic substance, e.g. the dead bodies of organisms, dung, milk, cheese, jam, leather, etc., or from humus (see p. I54). But this distinction is not absolute. For instance a parasitic fungus may begin its life in the living body of an animal or plant and continue to grow after the "host" is dead. Or a fungus which is ordinarily a saprophyte may, on occasion, attack 
the living body of an organism. A fungus which is strictly confined to parasitism is called an obligate parasite, one which can live either saprophytically or parasitically is a facultative parasite.

Moulds.-This name is commonly given to saprophytic fungi which grow on organic substances, provided they contain enough water, such as bread, jam, cheese, leather, tobacco, etc., on which they produce whitish, yellowish, greenish or bluish mycelia. They flourish particularly when the air is very damp and warm. In damp tropical climates any such substance will become covered with mould in a day, if it is exposed to the air.

Mucor.-One of the commonest genera of white moulds is Mucor, which forms a white, fluffy feltwork, rather like cotton wool in appearance, in and on the surface of such substances as those mentioned. If a piece of this feltwork is examined under the microscope it is seen to consist of delicate branched threads, forming the mycelium or body of the fungus. The mycelium of Mucor is a branched non-cellular tube, the wall enclosing the cytoplasm, which contains numerous very minute nuclei, though these cannot be seen except by special methods of staining. The cytoplasm encloses many vacuoles, which are smaller towards the growing tips of the hyphæ. The actual tip of each hypha is rounded and the protoplasm at the apex is without vacuoles. As the hypha grows in length the nuclei at the tip constantly divide.

The mycelium branches profusely, many of the branches penetrating the substratum on which the mould is growing, and absorbing liquid food. In this way it acts like the root of a higher plant, except that here the food is largely organic. The essential ele- 
ment carbon is taken by the fungus mainly in the form of a soluble carbohydrate such as sugar. Nitrogen, sulphur and the other necessary elements are absorbed in the form of various salts, together with water, although nitrogen is sometimes absorbed in the form of very complex compounds. The mycelium also branches in the air above the substratum, but its aerial growth is necessarily strictly dependent on the extent of the growth, and therefore of the absorbing surface, in the substratum.

Here for the first time among the types we have studied we meet with the fixed and branching habit characteristic of plants. The "food" of Mucor exists all round it in the bread or jam or other organic substances which forms its substratum, and the branching habit of its " root" puts it in touch with as much food as possible.

Oxygen for respiration is obtained from the air, and the necessity of free oxygen is illustrated by the fact that the mould forms a cushion only on and in the top layer of jam in the jam-pot. It is probable that the mycelium cannot normally penetrate into the deeper layers of jam because of the deficiency of free oxygen away from the air. Various species can, however, like yeast, live anaerobically for a time and can produce alcohol and carbon dioxide by splitting sugar molecules without oxidation.

So long as the fungus can get food, water and oxygen it continues to grow, i.e. the nuclei divide and the protoplasm increases in amount at the tips of the hyphæ. The wall covering the tip is continuously pushed out and new wall substance is continuously added to make good the thinning, just as in the growth in size of the embryonic cell of the 
higher plant (p. 107) Every now and then the growing hypha branches, i.e. a new branch of the tube is pushed out not far from the tip, and a new growing hypha is thus produced. This continuous indefinite growth and branching of the plant body is very characteristic of typical plants as opposed to the limtied compact growth of the typical animal (see Chapter I, p. 22). From what is known in the case of other fungi the growing tips of the hyphæ are probably sensitive to the stimuli of food substances and of free oxygen, i.e. they will turn and grow towards regions whence these substances are diffusing towards the hypha.

Reproduction.-(I) Spore formation. In place of the simple cell division of Amoba, Protococcus and bacteria, and the simple budding of yeast, the main method by which Mucor multiplies and produces new individuals is the formation of spores, produced in special chambers (sporangia) cut off from the general mycelium. Special hyphæ (sporangiophores) arise as branches of the mycelium and typically grow straight up into the air. These sporangiophores are positively phototropic, i.e. they grow towards brighter illumination. This generally involves growing away from the substratum, so that the sporangium formed at the end of the sporangiophore is placed in the best position for free distribution of its spores into the air.

The sporangium itself is formed by the swelling up of the tip of the hypha (Fig. I6, a), so that under a hand lens the sporangiophore looks like a minute pin with a globular head (the sporangium). A cross wall is formed, cutting off the head from the sporangiophore, and the protoplasm of the sporangium breaks up into a number of tiny multinucleate cells, each of which 
secretes a wall upon its surface. Directly the protoplasm of the sporangium breaks up, its turgidity, which has been maintained by the osmotic pressure in the vacuoles against the semi-permeable cytoplasm surrounding them, is destroyed, and owing to the osmotic pressure being still maintained in the sporangiophore on the other side of the cross wall, this is pushed into the cavity of the sporangium and pressed against and into the mass of spores (Fig. $16, b$ ). This pressure squeezes the spores against the wall of the sporangium ; and eventually, with the progressive drying of the wall by evaporation (since it is no longer in contact with the protoplasmic water), the wall becomes brittle and is burst, the spores being scattered into the air as a fine dust (Fig. I6, c).

The spores float in the air and are easily carried about by slight currents. The air of rooms and of towns generally contains innumerable spores of $\mathrm{Mucor}$ and other moulds, as well as bacterial cells and spores, and these gradually settle on all objects when the air is quiet. Thus any suitable organic substance, provided it is moist, will develop moulds or colonies of bacteria, or both, because the spores will germinate (or the bacterial cells grow) on such a substance from which they can get suitable food. The protoplasm of the spore absorbs water and food, the wall of the spore bursts, and the protoplasm, covered by a new delicate wall (Fig. I6, $d$ ), is pushed out, grows and branches to form a new Mucor plant.

Only the air of regions very remote from human dwellings, and from the substances on which moulds and bacteria thrive, is comparatively free from spores, and is in that sense pure. The purity of the air in this sense can be measured by the number of mould 
and bacterial colonies starting on a given surface of sterilised nutritive agar or gelatine which has been exposed to the air for a given time.

(2) Chlamydospore formation.-In some species of Mucor the protoplasm may contract into oval masses at short intervals along the length of a hypha (for instance a sporangiophore) the short diameter of the oval corresponding with or slightly exceeding the width of the hypha. Each oval mass becomes clothed with an independent thick wall, which often bulges out the walls of the parent hypha. The chlamydospores so produced become free by the breaking up of the wall of the now empty hypha, and are resistant resting spores which germinate to produce new plants under favourable conditions. Similar chlamydospore formation is not uncommon among fungi.

(3) Conjugation.-Quite a different sort of reproductive process sometimes occurs in Mucor. The tips of two hyphæ arising from the same or from different branches of the mycelium approach one another, and come into contact (Fig. I6,e), each swelling into the form of a club. A cross wall is formed towards the base of each club-shaped structure, cutting off a compartment at the tip (Fig. I6, $f$ ). One of these is frequently larger and more swollen than the other. The walls at the tips which are in contact are now absorbed and the cytoplasm of the two compartments mingles. The nuclei divide actively and then conjugate in pairs. The walls now swell up, forming a nearly spherical structure, and become dark-coloured, often black, with projecting excrescences on the surface (Fig. I6, h). Eventually the zygote (product of conjugation so formed) separates from the hyphæ on 
each side. The zygotes are 70 to $80 \mu$ in diameter and just visible to the naked eye. Inside the dark, rough outer wall are two layers of inner wall.

The zygote, in the protoplasm of which a considerable amount of organic food material, largely fats, is accumulated, is capable of remaining quiescent for a considerable time, the thick outer wall resisting desiccation. In a suitable medium it germinates, the thick outer wall bursting and the thin inner wall being pushed out by the protoplasm and growing into a new mycelium which often forms a single sporangium at once (Fig. I6, i).

The zygote of Mucor is a resting stage in the life history, preserving the plant through conditions unfavourable to active growth in the same way as the spores of yeast and bacteria; while the spores of Mucor, though also a resting stage in the sense that growth of their protoplasm is temporarily suspended, are primarily a means of multiplying and dispersing the individuals of the species, being formed in immense numbers and much less resistant than the spores of bacteria.

The process of conjugation, with the formation of a zygote, or product of conjugation, in Mucor is the first example of, this process we have met with. As we shall see in later chapters, this process is almost universal in the organic world, though in some of the lower forms it is apparently absent. In Mucor the two conjugating masses of protoplasm are often exactly alike, though in some cases one hypha is larger than the other. The conjugation of two equal protoplasts is called isogamy. $\mathrm{x}$

In some kinds of Mucor hyphæ from the same . Greck løos, equal, and $\gamma \alpha \mu \varepsilon \dot{\varepsilon} \omega$, marry. 
mycelium (even from the same branch) will conjugate, and in these zygotes are commonly found. In other kinds zygotes are much rarer, since conjugation will only occur between hyphæ of two different "strains," one of which grows more vigorously than the other. Here we have a functional differentiation between the conjugating hyphæ which is not quite on a par with, but shows an interesting parallel to, the ordinary sexdifferentiation, the essential characters of which we shall have to consider in Chapter XII.

Penicillium (Blue Mould).-This is a common form growing on substances similar to those on which Mucor occurs. It forms the well-known mould of Stilton and Gorgonzola cheese. The mycelium differs from that of Mucor in being septate, i.e. there are cross walls at intervals, dividing the mycelial tube into multinucleate compartments. The characteristic blue-green pigment is distributed through the cytoplasm, and has nothing in common with the chlorophyll of a green plant.

Spore Formation: Conidia.-Upright, aerial hyphæ arise from the mycelium as in $M u c o r$, but instead of forming sporangia, each forms several branches at the same level, the branches continuing the same general direction of growth but diverging slightly from one another. At a certain height each of these branches again in the same way, so that a sort of compound pencil of hyphæ is formed with the tips of their ultimate branchlets close together on the same level. From the tips of these branchlets minute spores called conidia are budded off, and before each is quite detached another is budded off behind it, so that a series of parallel or nearly parallel chains of conidia are produced (Fig. I6, j). These are very 
lightly connected, so that a slight jar or a current of air is sufficient to detach them, and they float off into

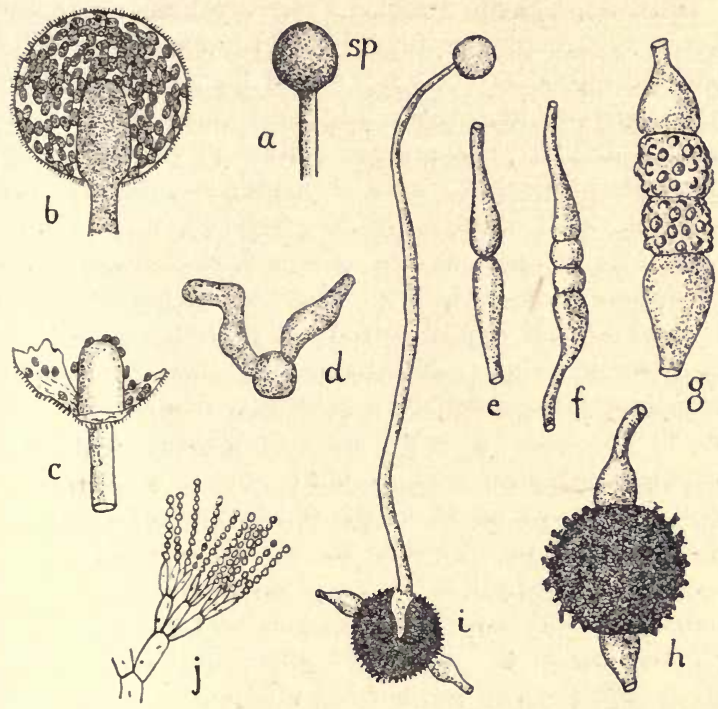

FIG. I6.-Reproduction of Mucor. (I) Spore formation $(a-d)$ : $a$, young sporangium $(s p) ; b$, optical section of mature sporangium (col., columella); $c$, remains of sporangium wall after bursting, with a few spores still adhering; $d$, germinating spore with two germ tubes. (2) $e-h$, Conjugation: $e$, conjugating hyphæ in contact ; $f$, separation of tips of conjugating hyphæ; $g$, later stage; $h$, mature zygote ; $i$, germination of zygote, germ tube forming a single sporangium.

Penicillium: $j$, part of branched conidiophore budding off chains of conidia.

the air, where they behave as spores, germinating on any favourable substratum on which they may happen to settle. 
There is also a complicated method of conjugation which is comparatively rarely met with and will not be described here.

Other Saprophytic Fungi.-There are numerous other forms of saprophytic fungi, including many with complicated reproductive bodies (" fruit bodies "), of which the common mushroom (Agaricus campestris) is a good example. The mycelium lives in the humus of. the soil, particularly in well manured pastures, and resembles that of $M u c o r$ in a very general way, except that it is septate, as are all the higher fungi. The mushroom itself is the fruit body, which arises first as a small weft of hyphæ forming a portion of the filamentous mycelium. This increases in size by constant branching and grows into a small very compact nodule, which increases in size and complexity, becoming differentiated into a short stalk and an arched top. Finally it grows up above the soil by the rapid elongation of the hyphæ forming the stalk and expands the umbrella-shaped pileus ${ }^{\mathrm{I}}$ or top of the mushroom. From the tips of the hyphæ which run at right angles to the surfaces of the platelike "gills" on the underside of the pileus there are budded off spores, four or two from each hypha tip, each formed on a small projecting point. The enormous number of spores produced by a single mushroom may be gauged by placing a ripe pileus, detached from its stalk, underside downwards on a piece of white paper and leaving it for a day or two. On gently removing the pileus a pattern of the gills will be found designed in streaks of microscopic spores on the paper. 


\section{PRACTICAL WORK.}

\section{MUCor.}

(I) Examine with a hand lens the young mycelium of Mucor growing in gelatine containing some nutritive substance such as raisin-extract. Cut out a very small portion of the gelatine containing the mycelium near its edge, and place it in a large drop of water on a slide till it becomes nearly liquid. Then put on a coverslip: squeeze it gently and look for hyphæ, first with the low and then with the high power of the microscope. Draw a portion of a hypha, including its tip, under the high power, showing wall, protoplasm containing vacuoles, which are smaller towards the tip. Note the absence of cross walls.

(2) Examine an older plant (if possible growing in a watchglass of gelatine) which has produced sporangia-first with a hand lens. Then place the watchglass on the stage of the microscope and focus slowly down with the low power. In this way an excellent idea of the habit of growth of Mucor is obtained. Note the spherical sporangia of different ages borne on the summits of the sporangiophores, and the branching mycelium below.

Mount some of the mycelium, carefully removed in a drop of methylated spirit to get rid of air, and add a drop of dilute glycerine. Find and draw various stages in the development of the sporangia.

(3) Examine a still older plant with mature sporangia. Mount some as in (2) and draw stages before and after bursting. Note the columella. Draw also a few spores.

(4) Examine a culture or a prepared slide showing zygotes. Look for and draw stages in conjugation and the formation of the zygote.

\section{Penicillium.}

(5) Examine Penicillium (Blue Mould) in the same way as Mucor-first in situ with the branched conidiophores rising into the air. Examine the brushes of radiating conidiophores under the low power. Examine part of the vegetative mycelium in gelatine squeezed out in water, and note the segmented mycelium with pale blue-green cytoplasm. Draw two or three segments under the high power.

Carefully remove some conidiophores, dip them in a drop of spirit, and mount in dilute glycerine; examine under the high power the chains of conidia and their mode of formation by budding from the tips of the conidiophore branches. 


\section{Demonstration Specimens.}

(6) Examine demonstration specimens of representative higher saprophytic fungi, such as the Mushroom, Puffball, etc. Note that the conspicuous part of the fungus above ground is a complicated spore-bearing fruit body. The mycelium lives below the surface in a soil rich in organic matter.

Examine (if available) stages showing the development of the mushroom from a small nodule on the mycelium. 


\section{CHAPTER XI}

\section{PARASITIC FUNGI}

A PARASITE, as we have seen (p. 78 ) is an organism which gets its food directly from another living organism. Bacteria living in the intestines are scarcely parasitic in the strict sense, though they actually live within the body of another organism. Many of them are harmless saprophytes living on the partly digested food of the animal, but some cause diseases because they produce toxins. Other bacteria, however, live in the tissues, which they break down by means of the enzymes they secrete, absorbing some of the products : these are parasites in the strictest sense.

Similarly with the fungi. Many are saprophytes: some of these, however, are able to attack and break down the tissues of living organisms (facultative parasites); others are exclusively parasites, and some are strictly confined to one species of host.

Fungi Parasitic on Animals. - Though the great majority of "zymotic" (germ) diseases of man and the higher animals are caused by bacteria or by protozoa (unicellular animals), e.g. malaria, sleeping sickness, dysentery, some-especially skin diseases-are caused by genuine fungi which form a mycelium.

The skin diseases generally known as ringworm are among the commonest of these. These are caused by several different kinds of fungi. Trichophyton, whose mycelium consists of chains of oval or rectangular 
cells 5 to $8 \mu$ in diameter, lives in and feeds upon the layers of living skin cells. These living skin cells are not on the actual skin surface, which consists of dead cells constantly produced by division of the living cells below the surface and rubbed off. The growth of the fungus often causes local inflammation, and the raising of the skin in circular pustules corresponding with the centres of infection. The mycelium grows outward from these centres, the central parts dying off and thus producing the ring-shaped structures characteristic of the disease.

Ring-shaped structures formed in this way are produced by many fungi, both parasites and saprophytes, for instance the "fairy rings" often seen in meadows, and also sometimes by the higher plants, for instance grasses, which similarly spread outwards from a centre by vegetative growth, the central parts dying off. The cause of the dying off of the older central parts of the plant may be either the exhaustion of the food supply, or the production of substances which act as a poison to the plant itself, in the same way that the accumulation of alcohol and carbon dioxide in fermenting wort eventually stops the growth of the yeast plant.

Another form of ringworm is caused by Microsporon, which attacks the living bases of the hairs of the skin, in which it spreads, forming a mycelium of rectangular cells, and causing the hair to break off short. The mycelium then breaks out to the surface of the hair, where it forms abundant spores $(2 \mu$ in diameter) by thickening of its cell walls. These spores are easily brushed off and render the disease extremely contagious. A number of allied species live on dogs, cats, horses, etc., and these are contagious to 
man, generally infecting the hairs of the scalp in children.

Other fungal diseases of the skin or mucous membranes are pityriasis, due to Microsporon furfur, which forms a spore-producing mycelium on the skin, especially in phthisical patients ; and "thrush," caused by a fungus which has a septate mycelium forming white patches on the tongue and on the mucous membrance of the throat, mostly in children, and also producing spores, round or oval in shape.

"Blastomycosis" of the lung or kidney is caused by single cells, which form endogenous spores, like yeast, and may also produce a mycelium in artificial cultures. This kind of fungus is also found on the skin, often following a slight wound, and causing suppuration (pus formation). Another fungus which inhabits the lung cavities is Aspergillus fumigatus, which forms a spore-bearing mycelium and spreads into the bronchioles (small branching air tubes of the lungs). This form is generally, but not always, found in company with disease-producing bacteria. Aspergillus also occurs in the external ear.

Saprolegnia and Fish Disease.-A good example of a facultative parasite is Saprolegnia, which lives saprophytically on the dead bodies or parts of animals and plants that have fallen into or died in the water, but which also attacks living aquatic animals, such as insects, fish and amphibia. A spore germinating on the body of an aquatic insect, for instance, sends out a tube which penetrates the tissues of the insect, and branches to form a mycelium which ramifies throughout the body. This, of course, causes the death of the insect, and the fungus lives saprophytically for a time on the dead tissues. Eventually thick branches 
of the mycelium are put out into the water, and each is cut off by a cross wall, forming a sporangium. The protoplasm of the sporangium then divides to form a number of spores. These are motile spores called zoospores, ${ }^{\mathrm{I}}$ oval in shape and with flagella attached to the pointed end. They are set free by the bursting of the sporangium wall, swim about in the water, and germinate, producing a new mycelium if they find a suitable organic substratum, living or dead. Sexual organs are also formed on the mycelium. These will not be described here, but it may be noted that, as is often the case in fungi which form sexual organs, conjugation may not take place, the female cell (egg) developing into a new plant without fertilisation. This behaviour is called parthenogenesis.

Saprolegnia sometimes attacks fish, particularly carp and gold-fish in stagnant ponds, entering by the gills, which it often blocks up, causing the death of the fish. According to some authorities it is a species of Saprolegnia which causes salmon disease, attacking the gills of the salmon, and sometimes killing them in great numbers, thus causing extensive damage to salmon fisheries. Other authorities, however, believe that bacteria are the primary cause of this disease in the salmon, which are attacked by Saprolegnia only when the fish are already weakened by the bacterial attack.

Fungal Parasites of Plants.-Fungi parasitic on the higher plants are far more numerous in species and far commoner than those which attack animals. They often cause widespread and serious damage to crop plants.

Pythium de Baryanum (causing the "damping off " of seedlings).-Closely sown young seedlings in a moist atmosphere, such as that of a greenhouse, some-

s Greek $\xi \tilde{\varphi} 0 v$, animal, from the motility of the spores. 
times topple over owing to the dying of the stem just where it emerges from the soil (Fig. I7, G). This is called "damping off," and is due to the attack of a fungus (Pythium) which has formed an extensive branching mycelium in the tissues of the seedling. The mycelium itself ramifies in the air spaces (intercellular spaces) between the living cells of the host (Fig. I7, A, B), but it pierces the living cells where it touches them (Fig. I7, B), breaking down their protoplasm. The seedling is soon killed and the fungus lives on for a time in the decaying stem: Eventually the ends of some of the hyphæ swell up to form a conidium (Fig. I7, A, sp., and C), which is cut off from the hypha by a cross wall, and becomes detached. In damp air this may grow out at once to form a new mycelium which penetrates a fresh seedling, but in water it puts out a short tube at the end of which a similar body is formed (Fig. I7, D). This is a zoosporangium (cf. Saprolegnia) whose protoplasm divides to form zoospores $(z)$, which on being set free by the bursting of the sporangium wall swim about in the water. The zoospores germinate $\left(z_{1}\right)$ to form new mycelia which penetrate fresh seedlings. Zoospores are never produced except in water.

Sexual organs are also produced. The female organ is a spherical structure formed by the swelling of the end of a hypha which is cut off from the rest by a cross wall. In this spherical structure the protoplasm contracts to form a smaller sphere $(e g g)$. The male organ is club-shaped, and is also cut off from the hypha by a cross wall (Fig. I7, E).

The male organ applies itself closely to the female organ (a), and a short tube from the former penetrates the latter (b). Through this the male protoplasm 

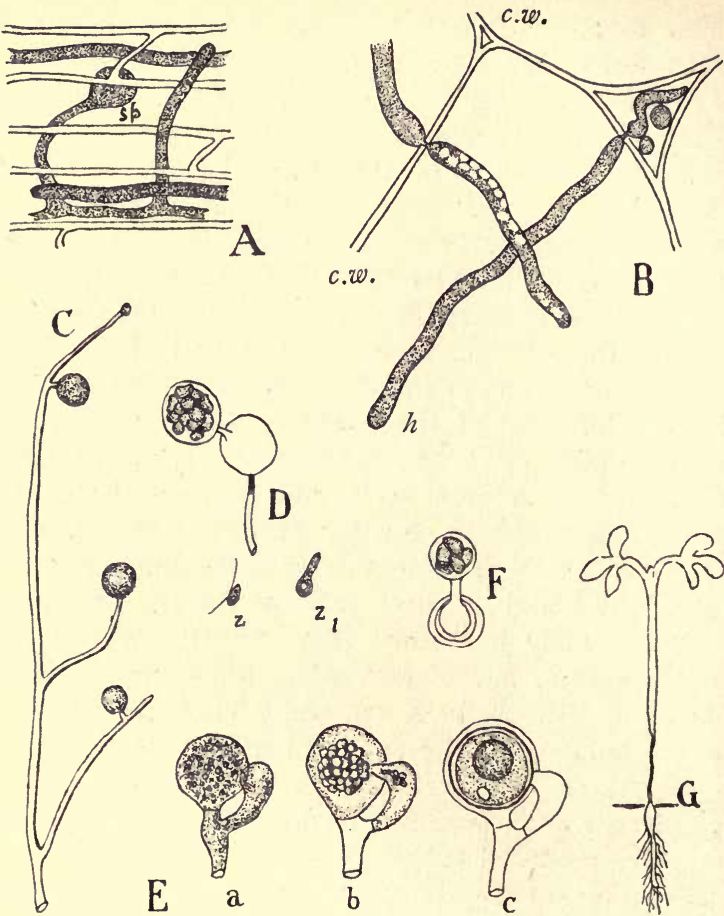

c.w.

B
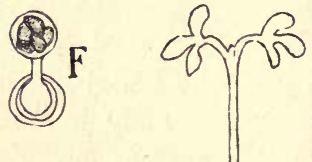

FIG. 17.-Pythium. A, hyphæ in tissue of seedling; sp., young sporangium. B, ditto, more highly magnified. The hyphæ $(h)$ have penetrated the cell walls $(c, w$.) through narrow holes which the growing tips have bored out. C, branched hypha emerged into the air, forming sporangia (sp.). D, germination of sporangium in water. The contents have divided to form a number of zoospores which are enclosed in a bladder pushed through a hole in the wall; $z$, free zoospore; $z_{1}$ germinating zoospore. E, sexual reproduction (conjugation); $a$, young female organ (spherical) and male organ (club-shaped) in contact ; $b$, penetration of male organ ; $c$, fertilised egg (zygote) surrounded by thick wall, male organ empty. F, germination of zygote in water (similar to D). G, cress seedling attacked by Pythium just above the soil surface. (After Marshall Ward, etc.) 
passes to coalesce with that of the female. The fertilised egg (zygote) now secretes a wall (c), and remains for a time in a resting condition. Eventually it germinates, forming zoospores in water $(\mathrm{F})$, which germinate, and if the hyphæ of this find a suitable seedling they penetrate it through a stoma just above the ground level.

The hyphæ of Pythium can only grow in air which is nearly saturated with water vapour, but these conditions are often realised between the stems of the seedlings growing in a seed-box which is frequently watered. When a seedling has been killed the hyphæ grow out from it and easily bridge the space to another seedling, which they enter and kill, and so on till the whole crop quickly becomes a rotting mass. The hyphæ of the mycelium are easily visible as very delicate white filaments, rather like cobweb, stretching between the seedlings.

Potato Blight (Phytophthora infestans).-This is a fungus closely allied to Pythium, and often causes enormous damage to the potato crop. Potato blight first appeared in Europe between 1845 and 1850 and ruined the Irish crop, causing serious and widespread famine. Since then it has always been with us, but its incidence is much more severe in some years than in others, according to the weather.

The mycelium ramifies in the tissues of the leaf, and the diseased patches turn brown as the tissues die. Under conditions favourable for the spread of the disease the whole potato haulm is soon reduced to a rotting mass. If the under side of a potato leaf bearing a patch of the disease is examined with a hand lens, the edge of the patch is seen to be covered with very delicate white glistening threads (the hyphæ) which have grown out through the stomata of the 
leaf (Fig. I8, F). Minute white bodies can sometimes be seen on the threads. These are the conidia, like those of Pythium, cut off from the ends of the hyphæ (Fig. I8, E, F, A). They are formed in great abundance in damp air and are easily carried by the wind from one plant to another. In water, for instance in a raindrop or dewdrop on a leaf, the contents of the conidium break up into zoospores (Fig. I8, B, C), as in Pythium, and each of these eventually germinates to form a hypha (D), which infects the leaf by growing in through a stoma or by piercing the cuticle. The conidia may also be carried down by rain into the soil, where they infect the young potato tubers which are growing beneath the surface. Whether the fungus can survive the winter in the soil and reinfect the seed tubers in the spring is not certainly known. One means of transmission of the disease from season to season is by mycelium carried over in certain of the tubers used for " seed." Such tubers are not sufficiently diseased to be detected, but in the early summer some of them may give rise to diseased shoots on which air-borne conidia are formed. These act as centres of infection to surrounding plants.

Phytophthora appears on the leaves of the potato plant in the damp south-west, for instance in southern Ireland, Cornwall and the Isle of Wight, as early as the end of May. From these regions it develops successively north-eastwards across the country. It usually appears in Cambridgeshire and Lincolnshire for instance -great potato-growing counties-about the end of July or the beginning of August, but its spread and the severity of the disease depend very largely on the weather. A warm, nearly saturated atmosphere, with constant south-westerly winds, are the conditions 
most favourable to the spread of the fungus, for in such conditions the conidia are formed, scattered by the wind, and germinate, with the greatest rapidity.
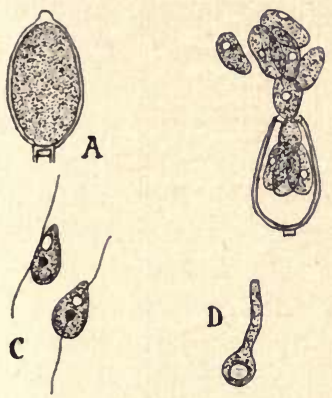

B
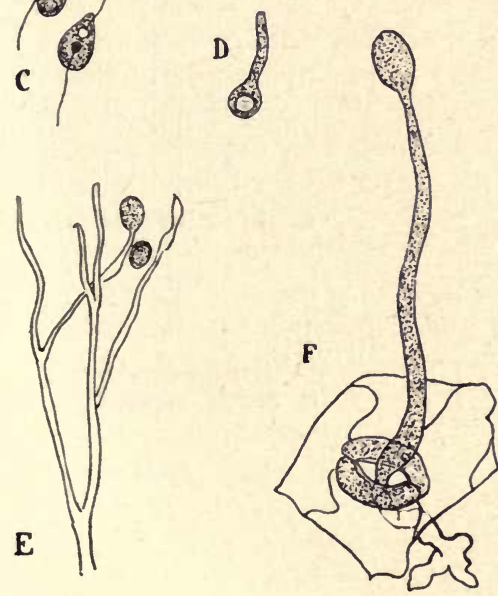

FIG. 18.-Conidia and zoospores of Potato Blight (Phytophthora infestans). A, conidium cut off from the end of a hypha. $\times 500$. B, conidium evacuating zoospores. $\times 500$. C. two zoospores. $\times 500 . \mathrm{D}$, zoospore germinating. E, branched conidiophore. The conidia have fallen from the tips of three branches, one is shown free and one still attached. The branch on the right is just forming two conidia. $\times 120$. F, young conidiophore protruding through a stoma and bearing a young conidium at its tip. $\times 200$. (After Frank.) 
In a dry summer the effects of the disease are usually negligible.

Potato blight cannot be cured once it has got a hold on the plant, but its spread can be very largely checked by spraying the leaves with "Bordeaux mixture." This is a "solution" of copper sulphate and lime; it dries on the leaves, and when wetted again by rain or dew it forms a poisonous copper solution which kills the zoospores, germ tubes and young hyphæ. In this way the crop can be very efficiently protected against the onset of the disease. Even if the disease is only moderately severe spraying will increase, or more strictly will prevent a diminution of, the potato crop. By destroying the zoospores and young hyphæ it enables the leaves to go on making sugar and proteins, essential for the growth and stocking with starch of the potato tubers, during August for instance, at a time when they might otherwise be injured and their chlorophyll largely destroyed by the fungus, even if the shoot of the plant were not entirely killed.

1 Rust of Wheat (Puccinia graminis and P. glumarum). -Puccinia graminis is an example of a highly specialised parasite with a much more complicated life history than that of Pythium or of Phytophthora. On the leaf or stem of the wheat plant straw-coloured or reddish streaks may appear in June or July. These are masses of uredospores, a special kind of conidia (Fig. I9, A), which are formed on the ends of hyphæ that have burst through the surface of the leaf or stem from a mycelium in the tissues below. Detached and blown about by the wind, the uredospores germinate

FIG. 19.-Life history of Rust Fungus (Puccinia). A, isolated uredospores of $P$. graminis (black rust). $\times 475 . \quad B$, germination or uredospore showing penetration of the germ tube through a stoma into the wheat leaf. Note the futile attempts at branching 

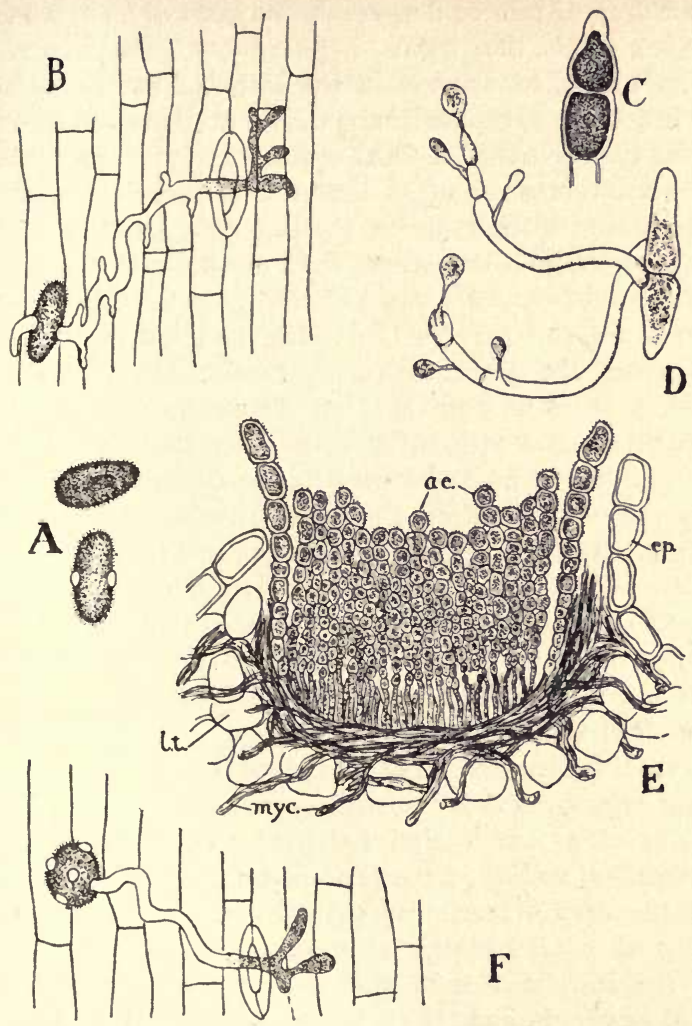

on the surface, and the aggregation of protoplasm in the branched apex. $\times 475 . \quad C$, single teleutospore. $\times 200 . \mathrm{D}$, germination of teleutospore of another rust fungus and formation of sporidia. $\times 475$. E, æcidium cup of another form in vertical section (cross-section of leaf) ; myc, mycelium of the fungus; $a$, æcidiospores; $l t$., leaf tissue ; $e p$. ., epidermis of leaf. (After De Bary.) $\times 150$. F, germination of æcidiospore on a grass leaf, penetration of a stoma by and branching of the germ tube. $\times 475$. (All except E after Plowright.) 
in a drop of rain or dew on the surfaces of fresh wheat leaves, and the germ hyphæ enter the stomata (Fig. I9, B), branch within the leaf, and penetrate the living cells. Hyphæ from this mycelium burst out from the surface of the leaf again, forming fresh masses of uredospores; and so the process is repeated, and the fungus spreads during the latter part of the growing season from one wheat field to another.

The uredospores formed about the time the crop is harvested can survive the winter and are capable of infecting the young wheat plants in the following spring. But another kind of spore, the teleutospore, ${ }^{x}$ two-celled and with thick dark walls (Fig. I9, C), are also formed in numbers towards the end of the season by the mycelium of Puccinia graminis (the Black Rust). The teleutospore hibernates, and in the spring, instead of gerıninating directly to form a new mycelium, sends out short thin hyphæ which cut off small thin walled conidia called sporidia (Fig. I9, D), and these do not infect the wheat plant, but instead the leaves of the barberry (Berberis vulgaris), a not uncommon shrub of hedgerows. The germ tubes from the sporidia form mycelia within the barberry leaf, and these form spores of a fourth kind (acidiospores) in cup-shaped structures (acidia) on the surface of the leaf (Fig. I9, E). At the base of each cup-shaped structure there is a mass of parallel hyphæ at right angles to the surface of the leaf, and from the end of each a chain of æcidiospores is budded off in much the same way that the conidia of Penicillium are budded off from the end of the conidiophores (p. I64). These æcidiospores are detached, and if they germinate on a wheat leaf

- Greek $\tau \varepsilon \lambda \varepsilon v \tau \eta \dot{\eta}$, end, because they are formed at the end of the season of active growth of the fungus. 
the germ tube penetrates a stoma (Fig. I9, F) and infects the wheat plant again, producing a mycelium which forms uredospores, the type with which we started.

Thus the fungus may live on alternate hosts (cf. the bacilli of tetanus and gas-gangrene, p. I55), the wheat and the barberry; though it may also, by wintering in the uredospore condition, live exclusively on the wheat plant. Long before this complicated life history was worked out, farmers had noticed that if there were barberry bushes in their hedges their wheat was particularly liable to " rust," and in some countries it is a legal offence to allow the barberry on a farm. Wheat rust often causes losses to the wheat crop of the world represented by millions sterling.

Puccinia graminis (the Black Rust) is rare on wheat in this country. Puccinia glumarum (the Yellow Rust) is much commoner, but, so far as is known, only two spore stages occur in the life-history of this species-uredo- and teleuto-spores.

There are thousands of different kinds of parasitic fungi known which infest wild and cultivated plants, some living exclusively on one species of host, some, like black rust of wheat, living on alternate hosts, and others being less exclusive in their habits. They often do severe and widespread damage to important crops and cause heavy losses in money. In the seventies of the last century, for instance, the coffeeplanting industry of Ceylon was literally destroyed by a fungus (Hemileia vastatrix) parasitic on the coffee plant. The study of the life histories and habits of these parasitic fungi forms one of the principal branches of plant pathology, which has become within recent years a study of great practical importance. For it is only by a careful and thorough study of the life 
histories and necessary conditions of life of the different fungal parasites that we can get the knowledge necessary to enable us to devise methods of preventing or mitigating their attacks. The cure of plant diseases caused by fungi is seldom possible; but preventive measures, taken after a thorough knowledge has been obtained of the parasite and of the conditions under which the disease spreads, are often very successful. Just as in the case of animal diseases, "prevention is better than cure," but with plants it is almost the only method which is of any use at all.

Bacteria are the cause of some plant diseases, just as we have seen that mycelial fungi cause certain animal diseases. But the latter are far more widespread as pathogenic plant parasites, just as bacteria are by far the most widespread and destructive of the parasites of animals. This difference is mainly due to two causes. First the spores of fungi are produced and carried about mainly in air, while many bacteria are largely carried about in the blood-stream which is absent from plants; and with this the second cause is connected. The life processes of fungi are adjusted to ordinary air temperatures, and the temperatures of the bodies of plants exceed these by very little. On the other hand, the life processes of pathogenic bacteria go on much more rapidly at higher temperatures, such as that which is maintained in the body of a warm-blooded animal (about $37^{\circ} \mathrm{C}$.).

\section{PRACTICAL WORK. \\ "White Rust" (Albugo).}

(I) The white pustules on the shoots of the Shepherd's Purse (Capsella) are caused by a parasitic fungus (Albugo) which often deforms the inflorescence. The mycelium of the fungus sends out hyphæ which bud off chains of conidia just below the surface of the host in such numbers that they burst off the surface 
layer of tissue. Scrape off a small portion of the pustule, mount in a drop of water and examine with the high power. Draw some of the spherical conidia formed by the club-shaped hyphæ (conidiophores).

(2) In cross-sections through a pustule (sections should be cut in alcohol and mounted in dilute glycerine) note the layer of closely packed chains of conidia perpendicular to the surface, and the mycelium in the tissue of the host.

(3) In sections through older pustules note the oogonia, each containing a fertilised egg (zygote) in the interior of the tissue of the host.

\section{Fungi Parasitic on Animals.}

(4) From the dead fly or ant's "egg" (pupa) infested by Saprolegnia" (which attacks both living and dead aquatic animals) scrape off a small portion of the surface, mount in a drop of water, and cover. Under the high power note the hyphæ, and the long club-shaped sporangia containing spores (which afterwards escape and swim in the water as zoospores, settling and germinating on other living or dead aquatic animals).

(5) If available, examine demonstration specimens of other parasitic fungi growing in the bodies of fish or of aerial insects (flies, caterpillars, etc).

(6) Examine slides showing the spores of ringworm (Microsporon) on the bases of human hairs.

\section{“True" Rust Fungi.}

(7) The wheat (or other grass) leaf provided bears orange or reddish pustules produced by the rust fungus Puccinia. Examine first with a hand lens and then place the leaf dry on a slide and examine with the low power. Note that the surface layer of the leaf is burst along the line of the pustule, which is formed of masses of spores (uredospores). Scrape off some of these and examine under the high power in a drop of water. Draw one or two spores and look for thin places in the wall through which germination will take place.

(8) Examine similarly the leaf-bearing "æcidium cups" formed by close-set chains of spores (æcidiospores) cut off from ends of hyphæ in the leaf. This is another stage of a rust fungus (Puccinia).

(9) Examine any fresh or museum specimens of other parasitic fungi that may be available.

' Easily obtained by keeping dead flies or ants' "eggs" in water brought in from a stagnant pond containing much organic débris. 


\section{ORIGIN OF SEX AND OF THE SOMA. THE GREEN ALGÆ}

LIFE probably began in the sea, and some at least of the earliest organisms were probably minute freefloating forms which were able to absorb and use light-energy to build up their bodies from simple inorganic substances, and must therefore be classed as plants. Of these some developed flagella and became actively free swimming, and their descendants are still represented by yellow, brown or green flagellate unicellular plants (algæ) which live in the sea or in fresh water. All these free-floating and free-swimming organisms, whether animals or plants, are collectively called plankton. ${ }^{I}$ One series of green flagellate plankton algæ, nearly all of which are confined to freshwater, illustrates very beautifully the way in which the differentiation of sex among conjugating cells came into existence, and the same series also illustrates the origin of what is called the soma, ${ }^{2}$ that is the body of an organism as opposed to its reproductive cells.

"Immortality" of Unicellular Organisms. - The body of a unicellular form, such as an amœba, a bacterium or a yeast plant, not only feeds and grows, it also divides (or buds) and produces new individuals of the species. This production of new individuals, or reproduction as it is called, is in origin simply an extension

Greek $\pi \lambda a \gamma \kappa \tau \delta$ s, wandering.

${ }^{2} \sigma \tilde{\omega} \mu \alpha$, body. 
of the growth process: it is discontinuous growth, as we saw in Chapter V, because it is growth conditioned by the separation of a part or parts of the individual to form new individuals. The protoplasm of the individual organism continues to exist and to increase in bulk, but it can only increase beyond a certain limit of size if it separates to form two or more new individuals. Under favourable conditions of life this process continues indefinitely, so that the protoplasm of which the organism is composed is immortal in the sense that it need never die so long as the conditions remain favourable to its continued life, though it dies of course as soon as the conditions become sufficiently unfavourable. But in the higher organismsin the great majority of multicellular animals and plants - the functions of nutrition and growth are, as we know very well, separated from the function of reproduction. The feeding and growing body does in most cases regularly die whether the general conditions of life continue favourable or not. It dies, as we say, of old age: it is a soma or " mortal " body. On the other hand, the reproductive cells (germ cells) which it produces grow, under certain conditions, into new individuals of the species. The organisms we are now going to consider will help us to understand how this separation of functions came to arise.

Chlamydomonas.-The organisms belonging to this genus are very common in pools, rain-water tanks, etc. Each is a minute green cell (Fig. 20, a), oval or sometimes rather oblong in shape, with a basin-shaped chloroplast occupying the hinder end of the cell, containing usually one conspicuous pyrenoid, and enclosing central colourless cytoplasm containing the spherical nucleus. The front end of the cell also consists of 
colourless cytoplasm to which are attached two long delicate flagella that protrude into the water in which the organism lives: by the rhythmical lashing of the flagella Chlamydomonas swims actively about, continually rotating on its long axis as it moves forward, like a rifle bullet in flight. The protoplasm of the cell is closely covered with a cellulose cell wall which thins away at the front end to which the flagella are attached. A red eyespot on the surface of the protoplasm, containing carotin (see p. II2), which renders

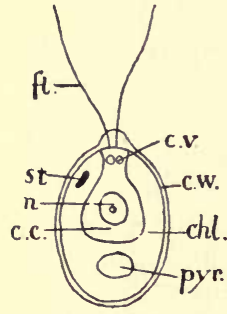

a

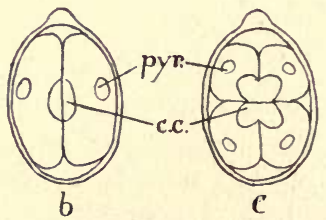

C

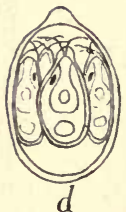

FIG. 20.-Chlamydomonas: $a$, diagram of swimming individual (vegetative cell); $b-d$, stages of division to form four new swimming individuals; $c . w$. , cell wall; chl., chloroplast; pyr., pyrenoid; c.c., central cytoplasm; $n$, nucleus; $c . v .$, contractile vacuoles; st., eyespot; $f$, flagella.

the organism sensitive to the direction of light, and two very small contractile vacuoles just below the insertion of the flagella, complete the equipment of the cell.

The nutrition of Chlamydomonas resembles that of Protococcus (p. 73). It can live and flourish in a weak solution of the proper inorganic salts (such as that given on p. I23), which provide it with the necessary elements for building up its protoplasm; and it makes sugar and starch (laid down round the pyre- 
noid) from the elements of carbon dioxide and water. It is in fact in its manner of feeding essentially a green plant, its constant active movement notwithstanding. We have every reason to believe that the main series of green plants are derived from similar motile forms.

\section{Reproduction of Chlamydomonas. - (a) Vegetative} Division.-In this, the commonest process of reproduction, the cell comes to rest, the protoplasm withdraws from the wall, the nucleus divides into two, and this process is followed by the division of the cytoplasm, including the chloroplast, a furrow appearing on the surface and extending inwards till separation is complete (Fig. 20, b). Sometimes the process of division stops there, and each new cell produces flagella and secretes a cell wall on its surface. The two daughter individuals begin to move actively, by lashing their flagella, within the cell wall of the mother, which they burst and then swim out into the water, leaving the empty shell of the mother cell wall behind. Each grows to the size of the mother individual. We see that this process is essentially the same as the division of an amœba into two new amœbæ, except that here the dead rigid cell wall is left behind. Under favourable conditions of life division takes place once every day, towards evening, and is complete in a few hours.

Very often, however, division does not stop with the first bipartition, but each daughter cell divides again (Fig. 20,c), so that four daughter individuals are formed instead of two (Fig. 20, d). These of course are correspondingly smaller, but on escape from the mother wall they quickly grow to the full size.

(b) Formation of Gametes and Conjugation.-Some- 
times the process of division continues further, so that by repeated bipartitions from eight to sixty-four cells are formed, their size varying inversely as the number produced. In some species they secrete cell walls, in some their bodies are naked, but in other

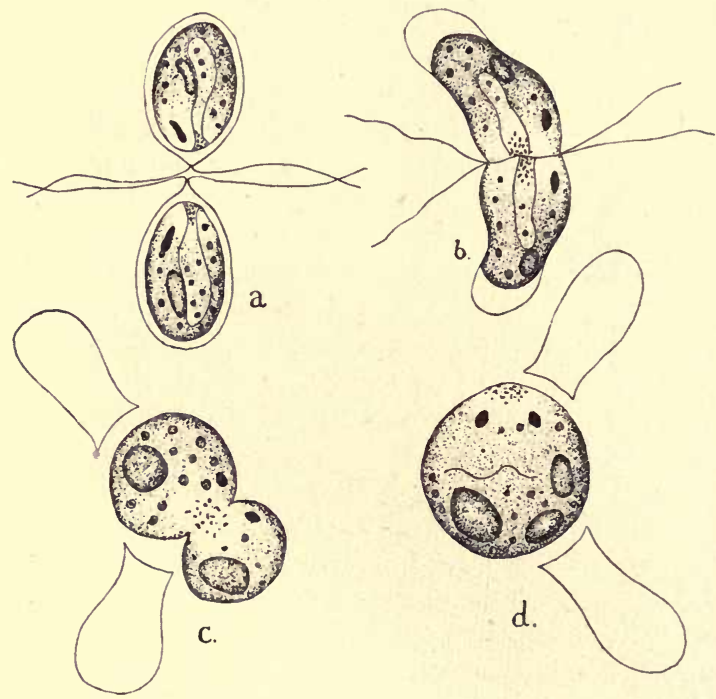

FIG. 21.-Conjugation of Chlamydomonas: $\boldsymbol{a}$, gametes approaching one another ; $b$ and $c$, stages of fusion; $d$, zygote. (After Dill.)

respects they are identical with the ordinary individuals. When these larger numbers of small daughter cells are produced, they do not, as a rule, at once grow into full-sized individuals, but conjugate in pairs, and are hence called gametes. I In the process of con-

- Greek $\gamma a \mu \varepsilon ́ \omega$, marry. 
jugation two gametes swim towards each other (Fig. 2I, a), their front ends come into contact, and their bodies gradually fuse completely (Fig. $2 \mathrm{I}, b$, Fig. 22, $a, b$ ), cytoplasm with cytoplasm, and nucleus with nucleus. When the gametes have cell walls the protoplasmic bodies slip out of them, leaving the
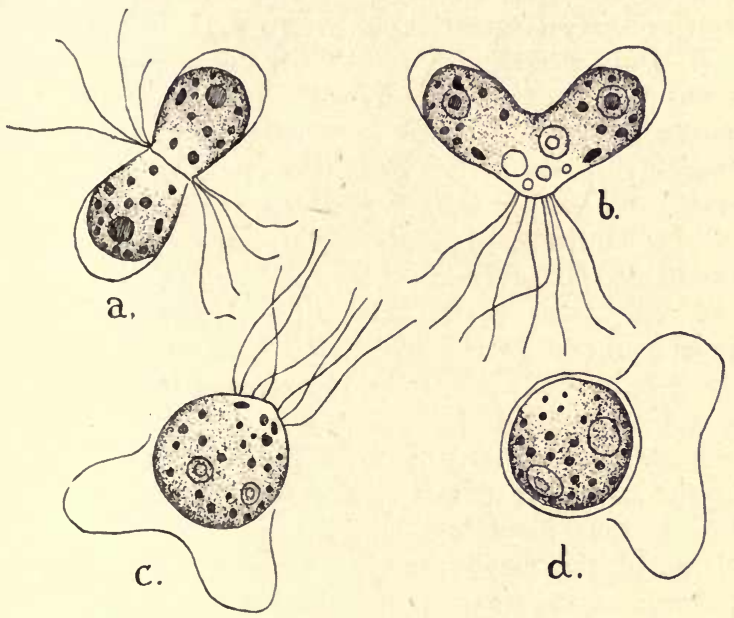

FIG. 22.-Conjugation of Carteria multifilis, a species with four flagella closely allied to Chlamydomonas: $a$, beginning of fusion; $b$, advanced stage; $c$, zygote with eight flagella; $d$, zygote with thick wall after loss of flagella. (After Dill.)

empty shells behind (Fig. 2I, c). The single cell formed by the union of the two gametes (Fig. $2 I, d$ ) is called the zygote (cf. Mucor, p. I62; Pythium, p. I75). In some cases the zygote continues to swim for a time with its four flagella, two derived from each gamete (Fig. 22, c-this is drawn from a form in which the 
single cell has four flagella and the zygote therefore eight), but eventually it loses the flagella, becomes perfectly spherical and secretes a thick cell wall (Fig. 22, d), going into the resting state, in which condition it can resist a certain amount of desiccation (cf. Mucor, Pythium, etc.). On germination the protoplasmic contents of the zygote, divide to form two or four ordinary (vegetative) individuals.

(c) Differentiation of Sex.-When the gametes are all equal in size, as they are in several species of Chlamydomonas, they are called isogametes. $\mathrm{I}$ But in certain species they are of two sizes, the larger derived from fewer, the smaller from a greater number of divisions of the mother cell. Such gametes are called heterogametes. ${ }^{2}$ Conjugation then, so far as has been observed, takes place, only between a large and a small gamete. In one species ( $C$. monadina), in which the details of the process have been carefully observed, and in which all the gametes have cell walls, the anterior ends of the two come into close contact, as in the case of isogametes, and the cell walls of the two become fused together (Fig. 23, a). The protoplasm of the small gamete separates from its wall (sometimes secreting a new wall round its hinder end, Fig. 23, B), and slips through the channel formed by the fusion of the two into the cavity of the large gamete, where it eventually fuses completely with the body of the larger, nucleus with nucleus and cytoplasm with cytoplasm-the chloroplasts with their pyrenoids remaining distinct longest (Fig. 23, c). The mass of protoplasm (zygote) so formed contracts, becomes spherical, and secretes a thick cell wall within the cell cavity of the large gamete (Fig. 23, c).

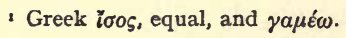

2 $\tilde{\varepsilon} \tau \varepsilon \varrho \circ$, other (different). 
Now, here we have two leading characters of the sexual differentiation of gametes, a difference in size and a difference in activity. Though both gametes begin life as free swimming cells of identical structure, one is more active than the other in the actual process of conjugation. This difference in activity seems here to be a mere mechanical result of the difference in size. If we suppose the protoplasm of each gamete

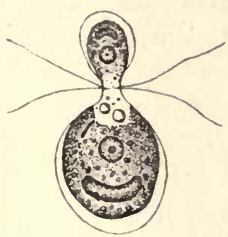

a

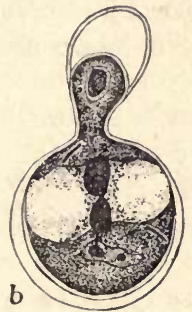

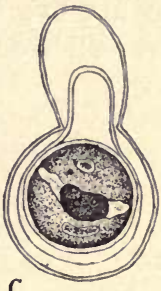

C.

FIG. 23.-Conjugation of Chlamydomonas monadina. a, Beginning of fusion of the small (male) and large (female) gamete. (Note that each has the normal structure of a vegetative Chlamydomonas-cell.) b, Further stage of fusion (drawn on a larger scale). Note the central area of colourless cytoplasm, derived from the front ends of the two gametes, and now containing the two nuclei (equal in size) in contact but not yet fused. c. Spherical zygote formed within the cell wall of the female gamete and itself clothed with a cell wall. Note that the two gamete nuclei have now fused to form the single zygote nucleus, but the chloroplasts are still separate. (After Goroschankin.)

to be attracted equally strongly towards the other, the body of the small one would be more easily drawn through the comparatively narrow canal formed between them; and this conclusion is supported by the unusual cases figured in Fig. 24. Here the gametes have come into contact obliquely, or have swung round after contact, so that no canal is formed, but the protoplasm of both slips out of its cell wall and the two form a zygote 
outside, just as in the case of the walled isogametes in Fig. 22.

In forms which show a more complete differentiation of sex, as we shall presently see, not only the size but also the structure of the two conjugating gametes is very markedly different, so that the large one (female) is necessarily quite passive, and the small (male) is alone active in the process.

We now have to consider a series of forms which do not live singly like Chlamydomonas, but in which the cells produced by ordinary division, each of which

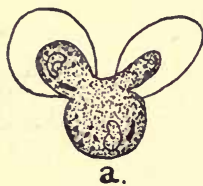

a.

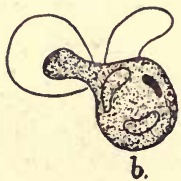

b.

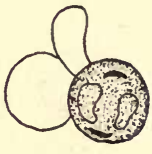

c.

Fig. 24.-Unusual type of conjugation in the same species. The gametes have met obliquely, and the protoplasm of both has emerged from the cell walls to form a spherical zygote outside.

has the same essential structure as a Chlamydomonas cell, remain together, surrounded by a common (mucilaginous) envelope, through which the flagella protrude. The colony or canobium ${ }^{2}$ of cells so formed behaves like a single organism, moving through the water by the co-ordinated beating of the flagella of all the cells. The first form we shall consider is

Pandorina, which is a spherical cœnobium of (usually) I6 cells pressed closely together, so that each cell is somewhat wedge-shaped (Fig. 25, A). In reproduction each cell of the cœnobium divides by 4 closely following bipartitions to produce 16 cells, and each group of 16 cells forms a new cœnobium (Fig. 25, B), the

s Greek Kotvós, common, and Blos, life. 

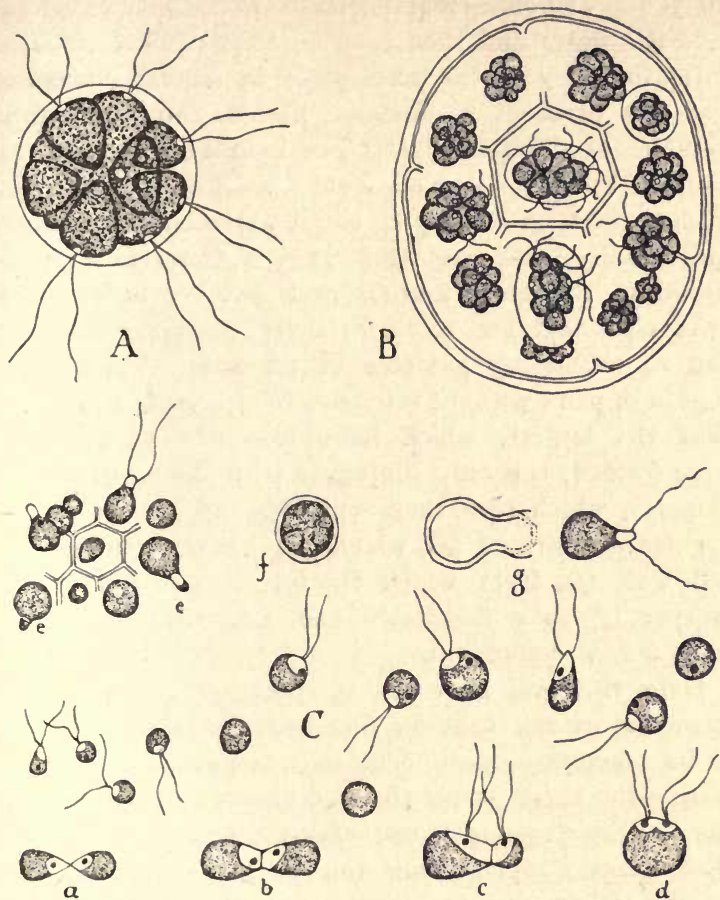

FIg. 25.-Pandorina. A, colony (cœenobium) of 16 cells in the vegetative state. $B$. reproductive condition-each cell of the cœnobium has divided to form a new daughter cœnobium. C, free-swimming gametes of various sizes; $a, b$, conjugation of gametes of equal size; $c$, of unequal size ; $d$, zygote still retaining the flagella of the gametes which formed it; $e$, conjugation between small (male) gametes which have originated elsewhere and large sluggish (female) gametes which have remained where they were formed by division; $f$, walled zygote; $g$, escape of contents of zygote as a free-swimming zoospore which will form a new cœnobium by division. 
I6 young cœnobia escaping from the mother envelope into the water and then growing to the full size. The formation of gametes takes place by similar divisions, but this proceeds to various extents, thus producing gametes of different sizes. The individual gametes so formed become loosened from the mother envelope, all but the largest escaping singly and swimming about individually in the water (Fig. 25, C). Gametes are produced at the same time from a number of cœnobia lying close together, so that the thin mucilage becomes full of swimming gametes of all sizes. These conjugate in pairs without reference to size $(a, b, c)$, except that the largest, which have remained where they were formed, can only conjugate with the small active gametes, which seek them out (Fig. 25,e). Spherical zygotes are formed $(d)$, which become covered with a cell wall $(f)$, from which the protoplasm eventually escapes $(g)$ as a flagellated cell, and this divides to form a new cœnobium.

Here then we have an even earlier stage in the evolution of sex than in Chlamydomonas monadinaan intermediate condition between isogamy and heterogamy-for there is no sharp division into two sizes, the largest gametes alone, which do not move from their places, representing the female condition, the smaller either acting as isogametes $(a)$ or as males $(e)$.

Eudorina and Pleodorina.-These are forms with spherical cœnobia, larger than Pandorina, and with all the cells (32 to I28) spherical and separate, forming a single layer on the surface of the cœnobium. Each cell is of the Chlamydomonas type of structure. In Eudorina all the cells of the cœnobium are alike and all take part in division to form new cœnobia, which takes place just as in Pandorina. The gametes are, 
however, strongly differentiated. The female gametes are very much like the ordinary vegetative cells except that they soon lose their flagella. They are not set free from the colony in which they are formed, which scarcely differs in appearance from a vegetative colony. The male gametes, on the other hand, are formed by division of each cell of a vegetative colony into 64 cells (male gametes) forming a plate, and each of these is long and narrow, tapering at the end, the green chloroplast being represented only by a yellow coloration at the hinder end. ${ }^{x}$ The plate of male gametes swarms out as a whole, like a cœnobium, but soon breaks up into its constituent gametes, each of which is capable of seeking out and conjugating with a female gamete, the process resulting in the formation of a zygote. Here we have the third character of sex differentiation-a difference in structure between the two gametes, as well as the difference in size and activity seen in Chlamydomonas monadina and to some extent in Pandorina. It is to be noted that the male gamete has suffered reduction in its nutritive equipment, i.e. its chloroplast, as well as in size.

In Pleodorina illinoiensis (Fig. 26, A) the cœnobium is almost identical with that of Eudorina, except that (usually) four of the cells at the front end of the cœnobium (which is often elliptical in shape) are smaller than the others. When division occurs to form new cœnobia these four smaller cells do not divide like the others; and they remain behind, eventually dying, when the daughter conobia escape. Here we have the first indication, in this series of organisms, of the appearance of a soma or mortal

- The male gametes of Endorina are very much like those of Volvox (see p. 200 and Fig. 28, D). 
body which takes no part in reproduction. It is difficult to say why these particular cells should be incapable of division. Perhaps it may be connected with a more highly developed function for perceiving the direction of light. It is generally true that specialisation of vegetative function in a cell carries with it loss of reproductive power. It is noteworthy that all transitions are found between the Eudorina condition, in which all the cells are equal and capable of

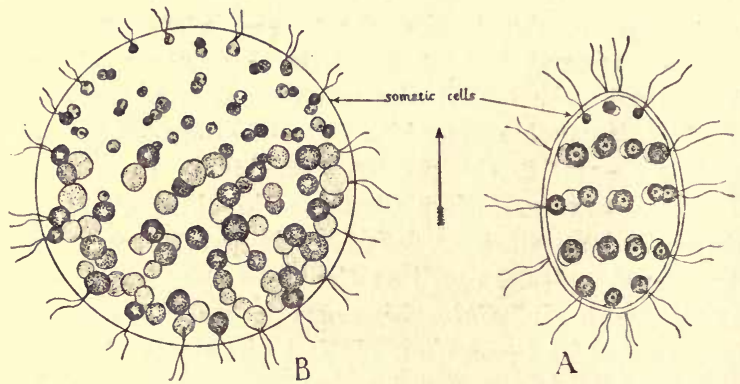

Frg. 26.-Pleodorina. A, P. illinoiensis. Cœnobium consisting of 28 large cells capable of division to form new cœnobia and 4 small cells at the front end of the colony which do not divide and die when the new cœnobia formed by the large cells are liberated. $\mathrm{B}, P$. californica. Sterile (somatic) cells more numerous. The arrow indicates the direction of locomotion in both cases.

division, and the occurrence of these sterile cells, which may vary both in size and in number from one to twelve, and that the capacity for division depends upon the size of the cell. Pleodorina illinoiensis is in fact considered to be a state of Eudorina, and thus we have the appearance of the soma, a fundamentally important step in evolution, first arising as a fluctuating condition within the limits of a species.

A larger form of Pleodorina $(P$. californica) has 
been described (Fig. 26, B), typically consisting of 128 cells, of which only about one-half, situated in the hinder part of the cœnobium, are capable of division, while the remaining cells in the front part of the

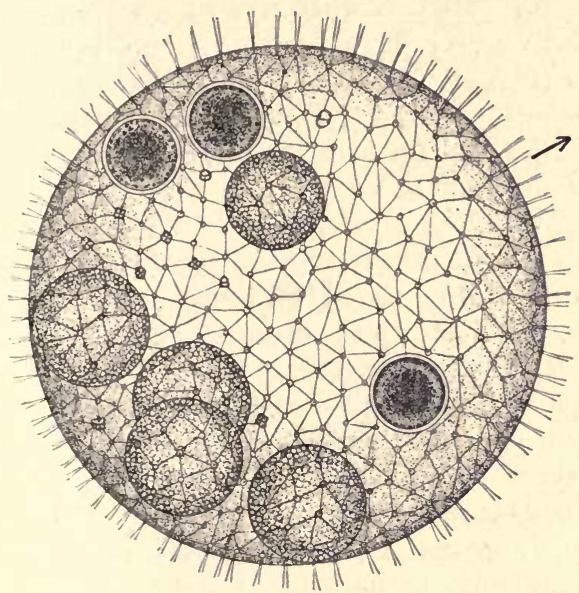

FIG. 27. Volvox aureus. $\times$ 180. (After Klein.) A cœnobium which has produced all three kinds of germ cells. The three large (walled) spherical cells are fertilised eggs, the small groups of cells are derived from androgonidia, which will divide further to form sperms (cf. Fig. 28, D), the largest spheres are young cœnobia derived from parthenogonidia; division in these last is complete, but the cells have not yet separated. Note that about a quarter of the mother cœnobium (the front quarter) is free from reproductive cells. The arrow shows direction of locomotion. Compare Fig. 26, A and B.

cœnobium are somatic, i.e. sterile and incapable of division. This marks a further step in the restriction of the reproductive (germ) cells.

Volvox.-In Volvox, the largest and most highly differentiated member of this series of forms, the cœnobium consisting of many hundreds of cells, the 
development of the soma has been carried much further. Here all the cells of the cœnobium are somatic (Fig. 27), i.e. purely vegetative in function and incapable of division, with the exception of a limited number of purely reproductive or germ cells. These germ cells are of three kinds: (I) so called parthenogonidia (Fig. 28, A), each of which divides to form a new cœnobium (Fig. 28, B), and thus corresponds in its reproductive function with the ordinary vegetative cells of a Pandorina or of a Eudorina; (2) gynogonidia (Fig. 28, C), which develop directly into passive (female) gametes (eggs) without flagella, these eggs being very large cells highly stored with food; and (3) androgonidia, each of which divides, as in the case of Eudorina, already described, to form a plate (Fig. 28, D) or a sphere of male gametes (sperms) I which have the same general characters as those of Eudorina. These three types of reproductive cell may be distributed in any combination. Thus a cœnobium may reproduce entirely by means of parthenogonidia, or it may produce eggs only, or colonies of sperms only, or any two, or all three forms of germ cell (Fig. 27).

There are two species of Volvox found in Britain, $V$. aureus (Figs. 27, 28, A-D) and $V$. globator. The former has rounded somatic cells which are separated from one another by considerable spaces, which are bridged by deli-

I Greek $\sigma \pi \varepsilon \rho \mu a$, seed. This is a useful term applied to all male gametes both in animals and plants; the male fertilising element has often been called the "seed" in common language.

FIG. 28. Volvox. A, portion of the surface of a conobium showing vegetative cells joined to one another by from one to three protoplasmic threads: also a parthenogonidium (non-sexual germ cell). $\times 550$, B, partly grown daughter conobium derived from the division of a parthenogonidium. $\times 550 . \mathrm{C}$, egg joined to neighbouring vegetative cells by bundles of protoplasmic threads. $\times$ about 700. D, isolated "plate" of male gametes (sperms) derived by division of an androgonidium. $\times 700$. 

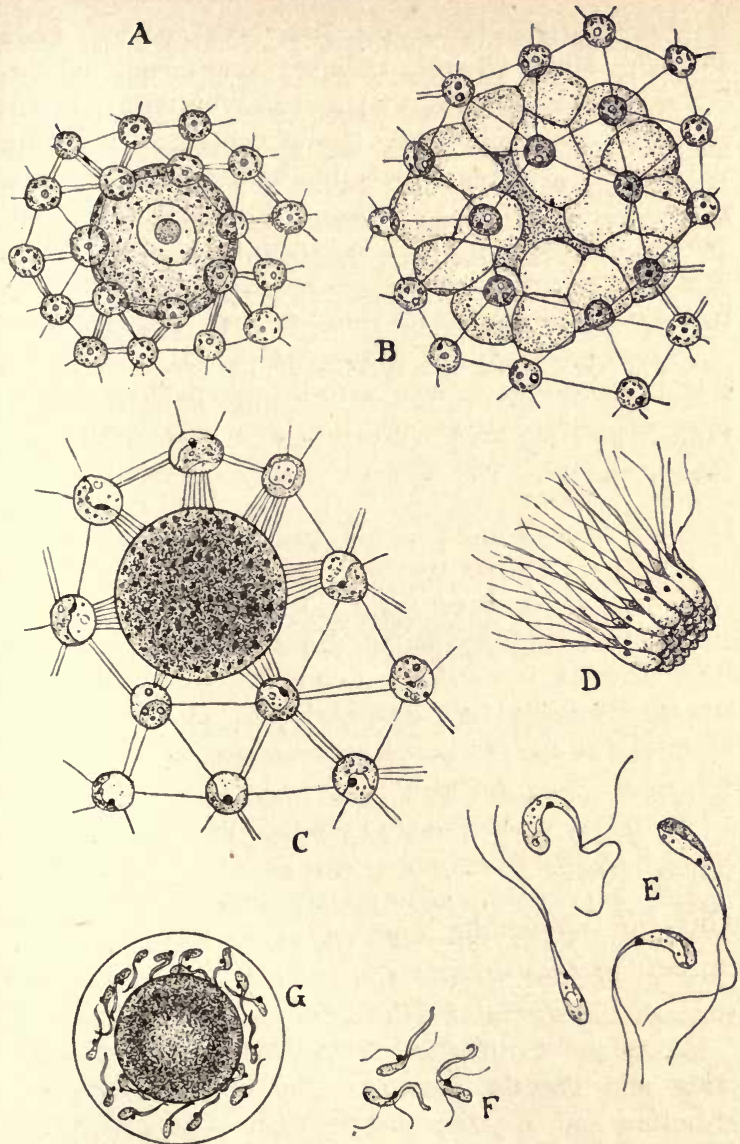

$\mathrm{E}$, isolated sperms of $\boldsymbol{V}$. aureus (note chloroplast, eyespot and flagella). $\times 825$. F, smaller sperms of $V$. globator on same scale (chloroplast degenerate). G, egg of $V$. globator surrounded by sperms. $\times$ 400. (After Klein and Cohn.) A-E, $V$. aureus. F, G, V. globator. 
cate threads of cytoplasm (Figs. 27, 28, A-C). V. globator, which is larger and less variable in size, has its cells much closer together and joined to one another by stout angular projections from the cytoplasm of the cell bodies (Fig. 29). It is interesting to note that $V$. aureus has larger green male gametes (Fig. 28, E), while $V$. globator has smaller very thin sperms, with the hinder end yellow and the two flagella usually attached about the middle of the body (Fig. 28, F), thus departing much further than the sperms of $V$. aureus from the structure of the primitive Chlamydomonadine cell, though still showing clear traces of derivation from that structure. The sperms of $V$. globator are nearly

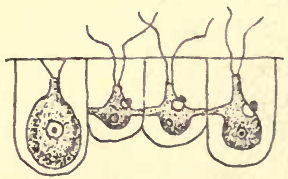

FIG. 29.-Vegetative cells of $V$. globator seen in profile of surface of cœnobium. Note cell walls and broad protoplasmic connexions. The cell on the left is a young parthenogonidium. $\times 1,600$.

as much reduced and highly specialised as those of green plants very much higher in the scale of vegetative structure. The extreme contrast between the male and female gametes in size, shape and structure is very obvious (Fig. 28, G), though each may be clearly derived from the Chlamydomonadine cell through the stages of differentiation we have traced.

Nature and Significance of the Differentiation between Male and Female Gametes.-The wide difference in structure and function between the male and female gametes of Volvox globator is repeated in the sexual differentiation of gametes in the vast majority of organisms, including all the higher forms of life, both 
animals and plants, and in nearly all the higher organisms - Seed Plants and a few other are exceptions-the male gamete or sperm is a small motile free swimming cell, while the female gamete or egg is a large passive spherical cell. The sperm is exceedingly sensitive, at least in a large number of cases, to chemical substances diffusing out from the female cell. Sperms are produced in immense numbers and only a minute proportion succeed in conjugating with eggs. The body of the sperm cell is reduced to the smallest sizecompatible with its function, which is to carry the paternal nucleus to the egg. Its chromatin contribution to the zygote nucleus is exactly equal to that of the egg nucleus (see Fig. 35, F, G). The hereditary characters are carried by the conjugating nuclei, and the paternal and maternal chromatin are equal in bulk and equivalent in effect on the characters of the offspring.

The egg, on the other hand, provides in the first instance the store of organic food substance with which the new individual produced from the zygote starts its life, and the size and passivity of the female gamete are correlated with this function. The fact that far fewer eggs than sperms are produced is also a result of this difference in size. The first beginnings of the differentiation we saw in Chlamydomonas and in Pandorina. The comparatively slight difference between the gametes in these forms appears as the result of what may be regarded as an accidental difference in the rapidity and duration of the process of division of the mother cell. The divisions of the mother cell of the smaller (male) gametes are more rapid and continue longer, so that smaller cells are produced, while the divisions of the mother cell of the female gametes is slower, so that larger cells are 
produced, the structure of the two being identical. The individual sluggishness of the larger gametes in Pandorina is probably merely a result of their larger size, but since they tend to remain passive and also contribute more cytoplasm to the zygote, we see here the foundations laid of the specific characters-the femaleness - of the egg in the higher forms. In these the differentiation is carried further and has become fixed-the smaller (male) gametes are no longer capable of conjugating with one another. They are shortlived cells, incapable of nourishing themselves, but extremely active and sensitive, their sole function being to get to the egg as quickly as possible and contribute their quota of nuclear material to the zygote. The female gamete has taken over entirely the function of feeding the new individual, and the amount of food that can be stored in it is not limited by the need of motility. This is a much more efficient arrangement for giving the new individual a good start in life than the conjugation of isogametes, both of which are motile and neither of which can contribute much food to the zygote.

The origin of sex is an excellent example of the origin of a differentiation which is at first, as it were, accidental, i.e. appearing without reference to its ultimate use, but is later fixed and further developed into an extremely efficient working mechanism. The more we learn of the evolution of structure and function in organisms the more we find that something like this is the history of the evolution of new characters.

In the higher forms the egg is often, as we shall see in the sequel, much reduced in size because parts of the parent organism take over from the egg the function of providing food for the new individual produced from the zygote. 
Besides the motile green algæ, some of which have been described in the preceding pages, there are many immotile unicellular forms, such for instance as Protococcus (Chapter IV). Some of these live on damp earth, tree-trunks, or in similar damp situations, while many float in water. Some are cœnobiate, like the Volvocines, and others form irregular loose colonies with an indefinite number of cells.

But there are also a large number of filamentous (thread-like) forms, the body commonly consisting of simple (Fig. 30, a) or branched (g) threads composed of cylindrical cells placed end to end and containing one or more chloroplasts of various shapes. Many of the filamentous forms which live in water are reproduced by the division of the contents of their cells into motile flagellate cells called zoospores (Fig. 30, $b$ ), of the same type of structure as the Chlamydomonas cell, but smaller and without a cell wall-cells in fact closely similar to Chlamydomonas gametes. These zoospores escape from the mother cell in which they were formed, swim about for a tmie $(c)$, and then settle down on some solid object and germinate, secreting a cell wall $(d)$, growing in length $(e)$ and dividing to form a thread of cells of the type characteristic of the species (Fig. 30, $f$ ). We may consider that in this form of reproduction the plant reverts to the condition of a Chlamydomonas-like ancestor for the purpose of reproduction: or to put the matter another way a Chlamydomonas-like ancestor settled down and divided without separation of the daughter cells from the cell wall, and then by growth and further division a thread of cells was produced, any of which at a later stage can produce a brood of Chlamydomonas-like cells which escape, and each of which reproduces the filament. 
The zoospores of such a filamentous alga may in certain cases conjugate instead of germinating at once, thus acting as isogametes. Such zoospores are called facultative gametes. But in most cases the gametes are
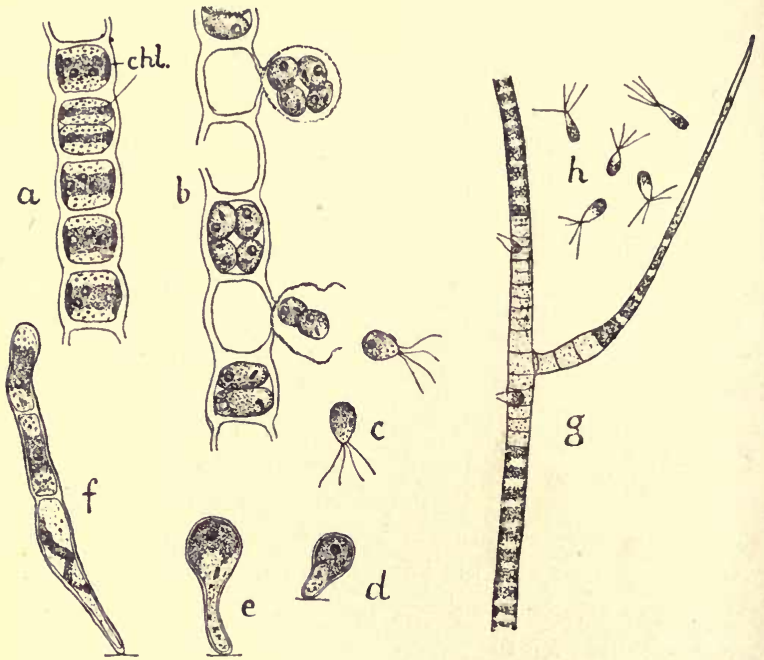

FIG. 30.-Filamentous green algæ: $a$, vegetative thread of Ulothrix with band-shaped chloroplasts $(c h l$.); $b$, thread whose cells are forming zoospores (like the individuals of Chlamydomonas but without walls); $c$, Free-swimming zoospore; $d$, germination of zoospore, which has lost its flagella, is attached by the front end to a solid object and has secreted a wall; $e$, the cell has grown in length; $f$, young filament derived from $e$ by further growth in length and transverse cell division (this grows into a filament like $a) ; g$, branched filamentous alga (Stigeoclonium), some of whose cells are vegetative, others have formed zoospores; $h$, free zoospores.

smaller than the zoospores, though quite similar in structure, i.e. more of the gametes, often double as many, are produced from the mother cell. Some 
filamentous forms have sexually differentiated gametes, and these in most cases show a wide differentiation between sperms and eggs, comparable with that seen in Volvox.

Sexual differentiation of gametes is found in various different groups among the algæ, evidently independently evolved along separate lines of descent. One may say that there is an inevitable tendency towards sexual differentiation, and towards the fixation of the extreme form in which the sperms are most widely different from the eggs. This high specialisation of the gametes, as already shown, is certainly the most efficient mechanism for the production of vigorous new individuals through conjugation.

The "Soma" in Plants and Animals.-The evolution of a soma or mortal body is beautifully illustrated in the Pandorina-Eudorina-Pleodorina-Volvox series. This evolution consists essentially in the separation of the vegetative and reproductive functions. In the lower forms of the series all the cells of the body discharge both functions, in the higher some cells discharge the vegetative, others the reproductive function. Directly we have any cells limited to the vegetative functions, we have a soma or mortal "body" by the very fact that these cells can no longer reproduce the species.

It must be noted, however, that this particular series of organisms is not in the direct line of evolution of any of the higher organisms. Volvox is the culmination of its own line of descent. It is probable that no further increase in size or complication is possible to the motile cœnobiate form of organism. The soma has been evolved on many other lines of descent from unicellular organisms. This particular line is chosen 
for illustration because of the existence of several forms which make up a closely connected series; and midway in this series Pleodorina shows the actual first appearance of the soma, $P$. illinoiensis being a very slight modification of the Eudorina type.

The vast majority of multicellular animals have a well-marked soma, i.e. a body consisting of tissues whose cells are not germ cells and do not reproduce the species by spore or gamete formation; but in many of the lower invertebrates new individuals are produced by budding of these somatic tissues which are not too highly specialised for particular vegetative functions. In the higher animals this process falls into abeyance, and the life of the somatic tissues is strictly limited to the service of the individual and comes to an end with the life of the individual.

In plants, however, the power of reproducing the species is much more often retained by the cells of the vegetative body. In the filamentous green algæ, for instance, referred to in the preceding section, all, or nearly all, the cells of the multicellular thread of which the body is composed retain the power of forming zoospores and gametes, and thus may become "germ cells." In the bulky algæ (seaweeds) with massive tissues, as well as in all the higher plants, this power is lost by the general body cells, but the plant may be reproduced " vegetatively" by budding, as in many of the lower invertebrate animals, new plants being thus formed apart from the germ cells proper. This power of "vegetative reproduction" is retained by some at least of the body cells of practically all plants, even the highest and most complicated forms, as we shall see in later chapters.

Taken together these facts show us that the dis- 
tinction between somatic and germ cells is not an absolute one. Though the germ cells (spores and gametes) are the specialised reproductive cells, whose sole function is to reproduce the species, this power may be retained by the body cells to a varying extent in different organisms. The body cells of plants retain it far more generally than those of animals, and this is undoubtedly connected with the fact that plant cells are, in general, far less highly modified for the performance of special functions than is the case with the cells of the higher animals.

Spirogyra.-This is a filamentous green alga belonging to a group which is distinguished by producing gametes that are not flagellated cells, conjugation taking place entirely within the mother cell walls. The body consists of an unbranched thread composed of cylindrical cells placed end to end. Each cell has a large central vacuole and a thin layer of cytoplasm lining the wall: in this is embedded a single chloroplast in the form of a green band which winds spirally round the cell from end to end (Fig. 3I, A). In the chloroplast is a row of pyrenoids. The nucleus is suspended in the centre of the cell. In other species several chloroplasts are present, running parallel with one another. The crossing lattice structure seen when the cell is looked through from the side is due to the fact that the parts of the chloroplasts which run round the further side of the cell are seen at the same time as the parts on the side towards the observer, so that the former appear to cross the latter. In a wide cell containing several chloroplasts, when the near side of the cell can be focussed alone, the far side being out"of view, it is clear that there is no actual crossing (Fig. 3I, B). In such a large cell the nucleus 


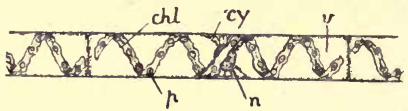

A

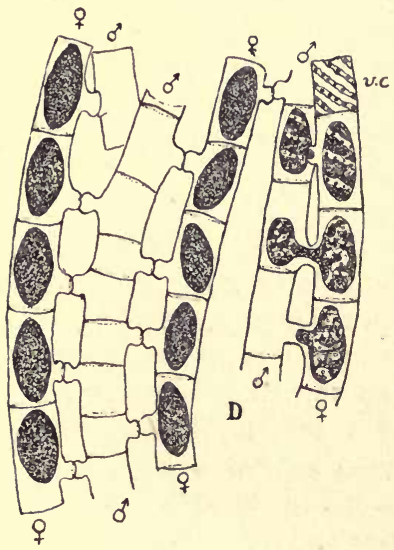

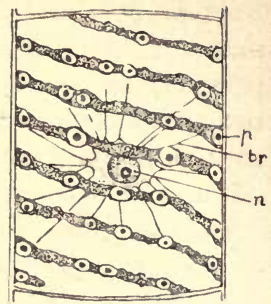

B

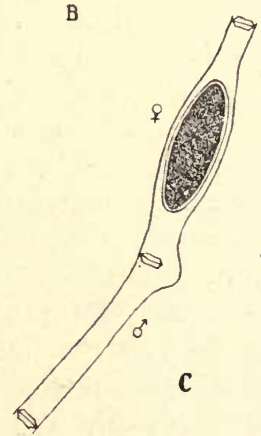

FIG. 31.-Spirogyra. A, one complete cell with parts of two adjacent cells of the filament of a species with one chloroplast in each cell ; $c y$., cytoplasm lining the wall; $v$, vacuole; $n$, nucleus suspended by a group of bridles; chl., spiral chloroplast; $p$, pyrenoid. $\mathbf{B}$, surface view of cell of large species with several chloroplasts, showing nucleus $(n)$ lying below, connected with surface by bridles $(b r$.$) which run to pyrenoids (p) . \quad \times$ about $550 . \quad \mathrm{C}$, zygote formed by conjugation of gamete formed in neighbouring cell (marked $\hat{\delta}=$ male) of the same filament with gamete formed in cell marked $o=$ female. $D$, five filaments which have taken part in conjugation. On the extreme left filament which has acted as female, and all of whose cells contain zygotes. Next is a filament all of whose cells have acted as male, alternate cells, conjugating with those of the filament on the left and on the right. On the extreme right are two filaments in process of conjugation-successive stages from above downwards (left, male; right, female). At the top of the right-hand filament a cell (v.c.) which has remained vegetative. (Modified from G. S. West. 
is suspended by long thin bridles $(b r$.$) , each of which$ runs into a chloroplast opposite a pyrenoid $(p)$. This is probably connected with the function of the nucleus in controlling nutrition (see p. 67), since it is round the pyrenoids that starch is laid down.

The cells of Spirogyra divide, after karyokinetic division of the nucleus, exclusively in the plane perpendicular to the long axis and halfway between the end walls, the new cell wall, secreted and constantly covered by cytoplasm, growing out in the form of a ring from the cylindrical wall, the effect being like the gradual closing of an iris diaphragm, till the two daughter cell cavities are completely separated. The cells then grow in length till they reach the length of the standard cell of the species. Under certain conditions the thread breaks up into lengths, or even into single cells, by the splitting apart of adjacent cells; and in this way the number of individual threads increases. Spirogyra is sometimes said to be "physiologically unicellular," because each cell functions as a self-contained unit-it is immaterial to its life whether it is isolated or whether it forms part of a thread.

\section{Gamete Formation and Conjugation.-Under certain} conditions two threads lying side by side form gametes, one from each cell. The cell walls of one of the threads on the side towards the other thread are thrust out in blunt projections, one from each cell, and the cell body of each cell begins to leave the wall. Almost immediately similar projections are thrust out from the cells of the other thread opposite those of the first set, the pairs of projections meet between the threads, the parts of the cell walls in contact are absorbed, and an open conjugation canal is thus formed 
between the two cell cavities. The cell bodies of the thread which began the process (male gametes) now slip through the canals and fuse with the bodies of the opposite cells of the other thread (female gametes), which have meanwhile contracted away from the cell wall, and a zygote is thus formed in the cavity of each female cell (Fig. 3I, D). The zygote becomes covered by a thick wall and ultimately germinates to form a new Spirogyra thread by splitting of the thick outer wall and protrusion of the inner thin wall (Fig. 32, C). Cf. the germination of the zygote of Mucor (p. I63, and Fig I6, i). In some species, however, adjacent cells of the same thread may conjugate (Fig. 3I, C), one acting as the male the other as the female cell, and in other cases, again, parthenospores may be formed, quite similar to zygotes in appearance and germination, but each produced from the contents of a single cell without conjugation.

The sexual differentiation of the gametes of Spirogyra is mainly seen in the active and passive rôles of the two conjugating cells: it does not involve a difference of structure or even of size. But in one species at least there is an interesting change in the structure of the male gamete after it becomes part of the zygote, its chloroplast degenerating and disintegrating in the zygote (Fig. 32, A, B), so that only the chloroplast derived from the female gamete remains and gives rise to the chloroplasts of the new thread formed on germination. This is a late occurring degeneration of the nutritive equipment of the male gamete, which may be compared with the reduction or disappearance of the chloroplast in the ordinary sperm before the formation of the gamete (cf. p. 200). Other slight indications of "maleness" may be found 
in some species, for instance the cells forming male gametes are sometimes shorter than those forming the
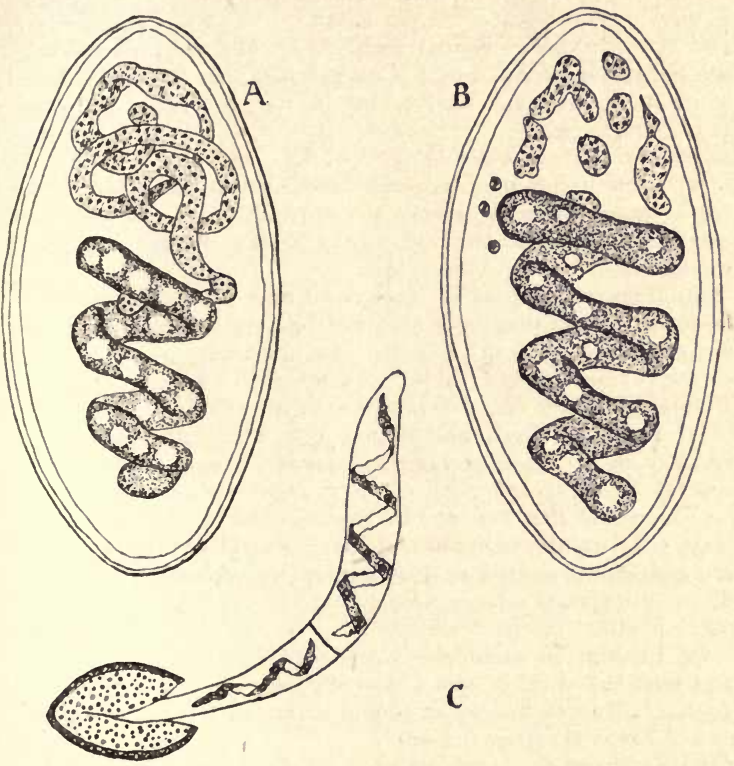

FIG. 32.-A and B, two stages of development of the zygote of a species of Spirogyra in which the wall is transparent and the chloroplast of the male gamete is degenerating and breaking up. The chloroplast of the female gamete alone produces the chloroplasts of the cells of the new individual. $\times 800$ (after Chmielevsky). C, germination of zygote: two cells of the new individual are produced. $\times 395$.

females, and in other cases the conjugation canal is mainly or even wholly formed by the male conjugating cell, the female cell merely swelling up to meet it. 


\section{PRACTICAL WORK.:}

(I) In a sample of Chlamydomonas note, under the low power, the moving green dots. Under the high power note in a specimen at rest (the flagella often get stuck to the slide or coverslip, and the cell thus anchored oscillates to and fro) the cell wall, the basin shaped chloroplast with pyrenoid, the clear front end of the body with the base of the flagella, and the red eyespot. Look out for stages of division. Run a drop of dilute iodine solution under the coverslip and observe again.

(2) In a living demonstration specimen under an apochromatic immersion lens observe the finer details of cell structure, including the nucleus situated in the colourless central protoplasm in the hollow of the chloroplast.

(3) Examine if possible Pandorina and Eudorina (preserved material if fresh cannot be obtained), noting the construction of the cœnobium and the fact that the structure of each cell is of the Chlamydomonas type. Examine also stages of division to form daughter cœnobia if these are available.

(4) Volvox. In $V$. aureus (on the whole the commonest species) note the large spherical cœnobia, each consisting of several hundred spherical cells. Compare several specimens, and trace the development of the daughter cœnobia from the large cells (parthenogonidia) of the mother. If sexual colonies are available note the development of the sperms by repeated division of special cells (androgonidia) and the large gynogonidia, each of which becomes an egg.

(5) Examine a single vegetative cell under the high power and note that it is of the Chlamydomonas type. Look for the flagella. Run in a drop of iodine and look for the threads of cytoplasm connecting the cells.

(6) Compare the structure of $V$. globator, and examine any demonstration slides of stages of development and details of cell structure that may be available.

- The material for this practical work may be varied according to what can be obtained. Chlamydomonas is generally available during the warmer months, and can in any case be kept in the laboratory without much difficulty. The cœnobiate forms are not so easy to keep in cultivation, and fresh material is by no means always available. For this reason it is advisable to keep a stock of material preserved in formalin, illustrating at least the vegetative structure of Pandorina, Eudorina, Pleodorina, and Volvox, and the formation of daughter cœnobia in these forms. Pleodorina can often be found by careful searching through samples of Eudorina. It is desirable also to prepare slides showing the formation of gametes, etc. 


\section{SPIROGYRA.}

(7) Draw a single cell $x$ on a large scale under the high power, showing cell wall, cytoplasm, chloroplast with pyrenoids, vacuole, nucleus (with nucleolus) and bridles (if present).

(8) Compare the pyrenoids of two preserved and decolorised samples, one of which, previous to killing has been well illuminated, the other kept for a day or two in the dark. Now stain each with iodine and observe again. Note that the pyrenoid itself stains brown with iodine (it is a protein crystalloid) as contrasted with the starch formed round it which stains dark blue (almost black).

(9) Examine the conjugation of Spirogyra, fresh if possible, if not in preserved material, and draw as many stages in the process of conjugation as you ean find.

(10) Plasmolyse vegetative Spirogyra ${ }^{2}$ with 5 per cent. calcium chloride solution (ordinary salt solution injures the cells) and draw stages in plasmolysis. Recover by placing in water.

I A species with a single chloroplast or two chloroplasts in each cell is the easiest to draw in the first instance. This should be compared with a large species such as $S$. crassa containing seven or eight chloroplasts, and showing bridles running into the pyrenoids.

If time permits. 
DIFFERENTIATION OF TISSUES. FUCUS : THE SEA-WRACK

Besides the Green Algæ, which are mostly unicellular or filamentous fresh-water forms (the "sea lettuce" is an example of a green alga which is marine and has a large thin body or thallus, which is soft and membranous, and coinposed of two layers of green cells), there are two other large groups of algæ, commonly called the Red and the Brown Seaweeds, because they are respectively red and brown in colour and they live in the sea, mostly in the intertidal zone or not very far below low-water mark. The plastids of the cells of these seaweeds contain the chlorophyll pigments, but in a different proportion to that in which they exist in green chlorophyll, and also other pigments in addition. For instance in the Brown Seaweeds there is a smaller proportion of the pure green pigments (chlorophyll $\alpha$ and $\beta$ ) and a larger proportion of the yellow and orange constituents (xanthophyll and carotin), and, in addition, a special orange pigment, fucoxanthin $\left(\mathrm{C}_{40} \mathrm{H}_{54} \mathrm{O}_{6}\right)$, peculiar to the Brown Seaweeds. This combination gives the phaoplasts, ${ }^{\mathrm{I}}$ as they are called, a brown or olive green colour.

The Brown Algæ vary from unicellular forms, through a considerable series of filamentous types, either con× Greek paúó, greyish. 
sisting of single rows of cells or of stouter threads several cells thick, to large forms ' with quite bulky bodies consisting of more or less differentiated tissues. One of these latter forms we shall now study in some detail, because it illustrates very well the fundamental principle of differentiation of function and corresponding differentiation of structure leading to the origin of distinct tissues.

Several different species of the genus Fucus live attached to rocks, mainly between tide marks, on the coasts of the cooler countries of the northern hemisphere. They are the commonest seaweeds on the rocky British coasts, and frequently cover the rocks so thickly as to make them very slippery to walk upon just after the tide has ebbed, leaving the mucilaginous surface of the seaweed wet and slimy.

The individual plants of Fucus vary from a few inches to several feet in length. The body or thallus consists of a cylindrical stalk or stipe attached to the rock substratum by a more or less branched spreading holdfast which cements itself firmly by means of the gluelike mucilaginous walls of the surface cells to the rock or stone on which the plant grows. Above, the stipe passes into the flat-forked frond by the gradual appearance of thin wings distinct from the thickened midrib, which is a direct continuation of the stipe. At the end of each branch of the frond is a groove or depression, often containing hairs consisting of single chains of cells, and it is by division of the cells at the base of the groove (Fig. 33, A) that the branches grow.

The end portions of some of the branches are swollen, and thickly set in the swollen frond are slightly

r Some of the Brown Seaweeds, for instance Macrocystis and Nereocystis, living in the Antarctic and Pacific Oceans, are immense plants, hundreds of feet long. 
raised papillæ, each of which is the projecting top of a spherical structure, hollow within, called a conceptacle. A minute hole leads through the tip of the papilla into the hollow of the conceptacle, which contains the sexual organs bearing the highly differentiated gametes. There are also (often) smaller, sterile conceptacles from which hairs protrude through the opening to the outside.

Thus in Fucus we have an external differentiation of parts or organs, though a very simple one: the holdfast which fixes the plant below, the stipe which is specially tough, and the frond which plays the chief part in photosynthesis and in growth; while the sexual reproductive organs are represented by groups of cells arising on what is really the surface of the frond, though the surface locally dips down, so to speak, to form the internal surface of the hollow conceptacle.

Microscopic Structure of the Thallus.-The minute structure of the different organs of bulky plants is best studied by examining under the microscope sections of the organ thin enough to be translucent when mounted in a liquid medium. To obtain complete information as to the structure of such an organ these sections have to be cut in different directions. The most instructive section is that taken at right angles to the axis of symmetry of the organ (transverse or cross section), for this displays the distribution of tissues about that axis. But the knowledge we gain from a transverse section must be supplemented by the examination of sections taken through and parallel with the axis (longitudinal sections), in order to study the structure of the tissues in longitudinal extension.

(I) Frond.-A cross-section of the middle of the frond shows three clearly marked regions: (a) the 
surface layer of cells (palisade or photosynthetic layer), (b) the larger isodiametric cells lying below (cortex), and (c) the central region of cells (medulla) the bodies of which are separated from one another by more than the thickness of an ordinary cell wall.

(a) Palisade Layer.- This consists of a single layer of cells (Fig. 33, A, $p$ ) whose long axes are perpendicular to the surface of the frond. Each cell of this layer essentially resembles a mesophyll cell (especially a palisade cell) of the leaf of a higher plant (Figs. IO, II). It contains a central vacuole and a peripheral layer of cytoplasm containing the nucleus and packed with phæoplasts. In this layer (as in the palisade layer of the mesophyll of a typical leaf) the greater part of the work of photosynthesis is carried on. These cells contain the greatest mass of phæoplasts, and the raw materials of the process (water, dissolved carbon dioxide and mineral salts) have direct access to them when the plant is covered by the sea at high tide.

(b) Cortex.-These cells (Fig. 33, A, c) are larger than those of the palisade layer, having larger vacuoles and fewer phreoplasts per unit bulk. The nucleus is often suspended in the vacuole by cytoplasmic bridles.

(c) Medulla.-The central region of the frond is occupied by cells (Fig. 33, A, me.) most of which are apparently isolated from one another. They are not, however, separated by air spaces like many of the cells of the tissues of a higher plant, but by a mucilaginous substance $(m u$.), which is really formed by the swelling of the middle layer of the joint wall between two adjacent cells. When these cells are first formed in development, the cell bodies are separated by thin walls, but the walls gradually increase in thickness and the middle layer becomes mucilaginous, takes up 
water and swells, forcing the cells apart and increasing the thickness of the thallus. The layers of wall on each side of this swollen middle layer, i.e. in direct contact with the cell bodies, also increase in thickness, more or less, but remain of firmer consistency, so that in the adult condition the cells appear isolated, each covered by a wall of its own (which may be thin or thick) and separated from its neighbours by a mucilaginous matrix. The medullary cells form chains or strands (like the threads of a filamentous alga) of cylindrical cells placed end to end. It is these chains which are separated from one another by the mucilaginous matrix derived from the middle layer of the original cell wall (Fig. 33, A). The cross walls separating the successive cells of a medullary strand remain thin. The structure of the body of a medullary cell is not different in essentials from that of a cortical cell, that is to say there is a central vacuole and peripheral cytoplasm with nucleus and phæoplasts, but these last are often very sparsely scattered.

The direction of the medullary strands differs as between the wings and the midrib : in the former they run horizontally or obliquely, in the latter longitudinally, so that they appear in cross-section as circles. Groups of cells at the outer edge of the medulla of the midrib, just below the cortex, have especially thick tough walls, and these may be called fibres, by analogy with the somewhat similar longitudinally running thick walled cells of the higher plants. Like them they increase the toughness of the thallus. The main function of the medullary cells is probably that of conduction of organic food substances tormed by the photosynthetic layer to the regions of growth at the apex of the frond. 
In longitudinal sections of the frond the palisade and cortical cells appear very much the same as they do in transverse sections, but the medullary cells of course look different, those of the midrib being now cut in longitudinal section, i.e. showing the length of the chains, instead of as single cells cut transversely.

(2) Stipe.-The stipe consists of cells similar to those of the frond, but with some notable differences. There is no single layer of surface cells overlying a distinct cortex, but several layers of cells in radial rows, the cells of each row separated by thin walls, indicating division parallel to the surface of the frond (tangential division). The surface itself is rough owing to the knocking about the stipe suffers from the waves against the rocks, and often shows radial splits. The medulla, which forms much the greatest bulk of the stipe, consists largely of fibres, with a few wide cells having dense vacuolated contents, including a number of phæoplasts and fairly thick walls. These may perhaps serve to conduct organic food from the frond down to the holdfast, which increases in size, sending out fresh short branches as the plant grows. As the base of the stipe is approached the thick-walled fibres increase and the holdfast itself consists exclusively of them.

Apical Growth and Nutrition.-The basis of growth in Fucus, as in the higher plants, is active cell division at the tips of the branches. At the bottom of the apical groove of each branch there is situated a comparatively large six-sided, box-like cell (Fig. 33, A, a), with one side forming part of the surface of the base of the groove. This apical cell gives rise by its divisions to the cells of all the tissues of the branch. The apical cell is constantly dividing unequally, cutting off 
a flattish oblong cell from each of the four sides and from the base (towards the centre of the frond) in turn. After each division it grows to its original size. The cells cut off divide further and form an almost homogeneous small-celled thin-walled tissue, densely filled with phæoplasts, in the whole region of the branch apex. As one passes away from the apex the different tissue regions quickly differentiate (Fig. 33, A). The surface cells do not change their character much and form the photosynthetic layer. The cells lying next below increase in size by swelling of the vacuole, but do not increase their cytoplasm. These cells become the cortex. The cells towards the centre of the frond grow in length very considerably and separate laterally by the swelling of the middle lamellæ of their lateral walls, as already described, thus forming the medulla. The strands of medullary cells are also passively stretched owing to the elongation of the frond by the continued division of the surface cells at right angles to the surface plane.

It is clear that at the apices of the branches there is constantly going on a great increase in the bulk of protoplasm and of cell wall substance, and this requires a continuous supply of soluble carbohydrates and proteins or other nitrogenous organic substances. Some of this organic food will no doubt be supplied by the synthesis of these substances from carbon dioxide, water and salts absorbed by the surface layers of cells close to the apex, which contain numerous phæoplasts. But in an actively growing plant the consumption will be much greater than this supply, and the balance required must come mainly from the palisade layer of the mature parts of the frond. The easiest channels for this flow of substances are neces- 

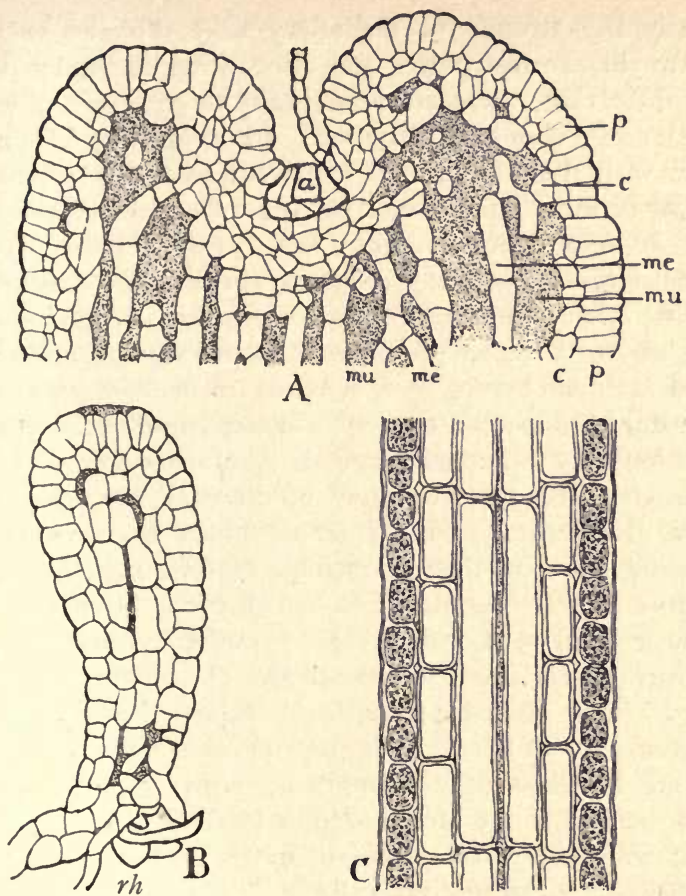

FIG. 33-A, longitudinal section through the apex of frond of Fucus (the cells are in outline, the intercellular mucilage dotted); $a$, apical cell surrounded by the cells produced from its previous divisions; $p$, photosynthetic layer; $c$, cortex; me., medullary cells (mu., mucilage formed from their walls). Further from the apex the cortical cells increase in size and the medullary cells in length. B, young plant of Fucus in the cylindrical stage. Apical groove just developing. Medullary cells beginning to lengthen and to separate; $v h$., first rhizoids which develop into the " holdfast." C, longitudinal section of part of the bristle-like thread of Stictyosiphon ( 6 cells thick) showing dense protoplasmic contents with phæoplasts of surface cells (photosynthetic layer), which are half the length of the next layer (cortex). These, again, are half the length of the central cells (medulla), which are slightly separated longitudinally. This arrangment is due to the more numerous divisions of the surface cells. Compare B. 
sarily the strands of medullary cells, because these form the shortest paths, and also, owing to the length of these cells, there are fewer walls to pass in a given distance. The medullary cells are connected at one end with the cortex, and through it with the palisade layer of the mature frond; at the other with the mass of embryonic cells lying behind the apical cell. Hence these medullary cells may be called conducting cells. The diffusion of substances from a region of higher to a region of lower concentration, in plant and animal tissues for instance from a region of constant production to a region of consumption (i.e. conversion into other substances), is a necessary consequence of the physical laws of diffusion, though the rate of diffusion and the actual paths it takes will depend on a number of variable factors, such as the nature of the substances and the obstacles to diffusion. The cortical cells will act to a certain extent as a storage tissue, since if the stream of soluble organic food from the photosynthetic layer through the cortex and medulla to the growing points is checked, owing to the supply being temporarily greater than the demand, some of the surplus food will be arrested and will tend to accumulate in the cortical cells as well as in the medullary cells.

Sexual Reproduction.-Unlike the plants hitherto considered Fucus reproduces itself exclusively by means of gametes, and these show high sexual differentiation, comparable with that of the gametes of Volvox (cf. Figs. 28, G, and 35, D). The two kinds of gametes are produced in the cells of the sexual organs arising on the inner surfaces of the conceptacles. In some species the male and female organs are formed in the same conceptacle in others in different concep- 
tacles which may be produced on different individual plants.

The female organ (oogonium) arises from a single cell on the surface of the conceptacle. This grows up to form a papilla which is cut off by a cross wall at the base, and then divides transversely to form a stalk cell and a body cell (Fig. 35, A). The latter becomes large and spherical and its nucleus divides into eight by successive bipartitions. These eight nuclei have different fates in different species. In some the cytoplasm divides correspondingly and eight eggs are formed in the oogonium (Fig. 35, B), but in others four or six of the nuclei degenerate and disappear and the whole of the cytoplasm forms four or only two eggs. In others, again, seven of the eight nuclei degenerate and only one egg is formed from the whole of the protoplasm of the oogonium. ${ }^{\mathrm{I}}$ The oogonium wall has two layers: the outer bursts, setting free the inner wall as a bladder enclosing the eggs (Fig. 35, C).

The male organs (antheridia) are club-shaped cells (Fig. 34, A, a), which are branches of a hair that arises, like the oogonium, from a surface cell of the conceptacle. The nucleus of the antheridial cell divides by repeated bipartitions to form 64 nuclei (Fig. 34, C, n), and the cytoplasm divides correspondingly so that 64 sperms are formed (D). Each of these is a minute biflagellate pear-shaped cell containing besides the nucleus (Fig. 34, D, E, n) a single orange phæoplast. The mass of 64 sperms is freed from the

s This process is essentially the same as the formation of the socalled "polar bodies" of animal eggs. In the animal a brood of four gametes is formed from the mother cell, of which three degenerate (polar bodies), leaving a single egg. In Fucus and allied genera a brood of eight gametes is formed, of which all are fertile eggs, or in some species four, six or seven degenerate. 
antheridial hair enclosed in the inner wall of the antheridium.

The bladders containing the eggs and sperms are
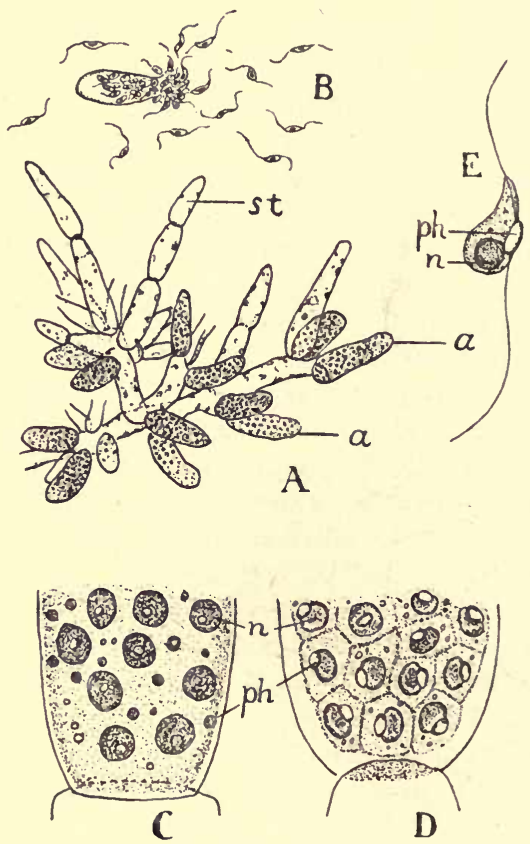

Frg. 34.-A, branched hair from conceptacle of Fucus showing antheridia $(a)$ and "sterile" cells (st.). B, bladder, formed by inner wall of antheridium, bursting and setting free the sperms (male gametes). C and D, stages in development of antheridium. E, free male gamete with two flagella; $n$, nucleus; $p h .$, phæoplast.

forced through the pore forming the mouth of the conceptacle by the pressure of the swollen walls of the hairs which fill up most of the space within its 
cavity. They are expelled from the conceptacle at low tide when the plants are exposed to the air, and lie in masses in the film of water adhering to the slimy surface of the frond. The masses of sperm bladders, where these are separate from the eggs, can be easily distinguished with the naked eye by their bright orange colour due to the orange phæoplasts of the sperms. The bladders enclosing the sperms and eggs then burst (Figs. 34, B, 35, C), setting the gametes free in the film of water.

The spherical egg contains much food material and is many times the diameter of the sperm. It secretes a substance of unknown nature which attracts the sperms, and these, usually in considerable numbers, cluster round it (Fig. 35, D), and by their constant oscillation frequently set up a vortex in which the egg rotates. One of the sperms eventually penetrates the egg and the sperm nucleus travels across the cytoplasm and fuses with the egg nucleus (Fig. 35, E, $\mathrm{F}, \mathrm{G})$. Though the sperm nucleus is much smaller than that of the egg, the amount of chromatin it contains is seen to be equivalent in bulk, as is always the case.

Development of the Young Plant.-The fertilised egg (zygote) germinates at once. A cell wall is secreted, the zygote lengthens, one end becoming pointed, and the nucleus divides by a cross wall; the cell corresponding with the pointed end forming the first attaching organ or rhizoid, ${ }^{\mathbf{I}}$ which sticks to any solid substratum with the help of its mucilaginous wall. The upper cell divides repeatedly, and this part of the plant body elongates into a club-shaped form (Fig. 33, B), fresh rhizoids meanwhile growing out at the base. Very soon an apical cell is established on

. Root-like organ, Greek $\rho l \zeta a$, root. 

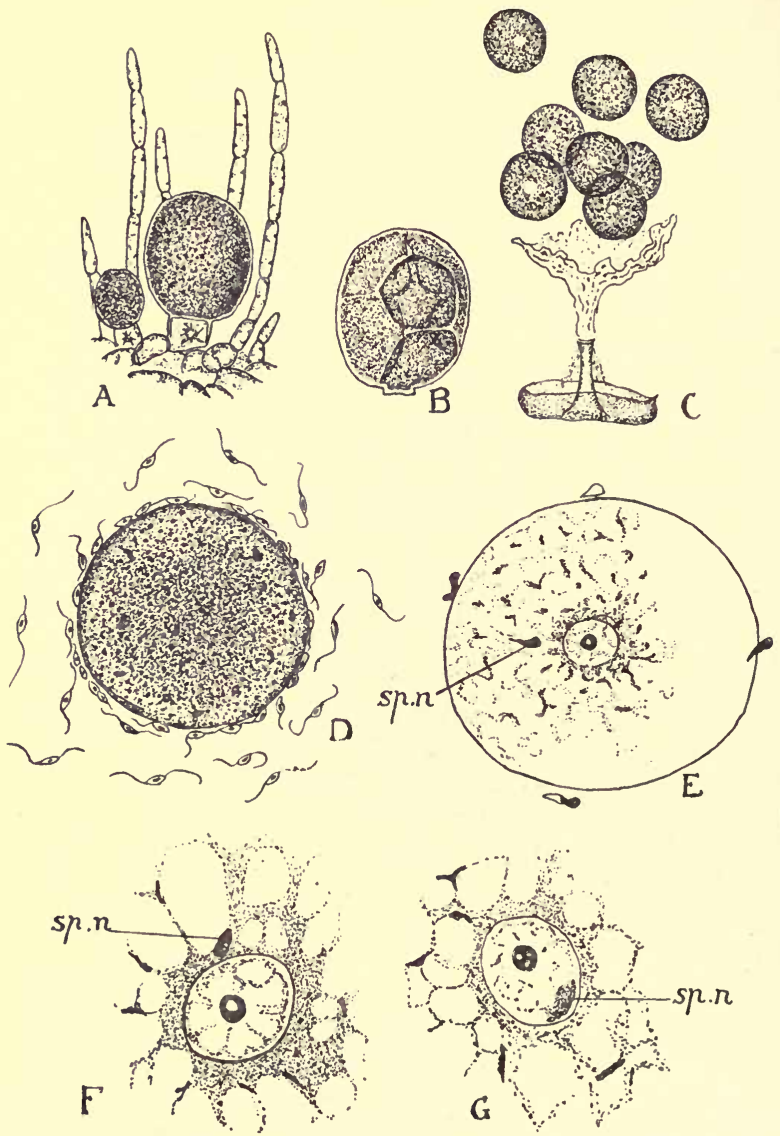
the surface in the centre of the upper end, and by the more rapid growth and division of the neighbouring surface cells the apical cell shortly becomes sunk in a hollow. Up to this point the young plant is nearly cylindical in form, but now the upper part grows more quickly in one longitudinal plane than in the plane perpendicular to it, and thus the wings of the frond are started.

A marked difference is soon apparent between the growth of the surface cells and those occupying the centre of the thallus. The former remain small, divide actively and form the palisade (surface) layer. The cells lying immediately below do not divide so rapidly and form the cortex. The central cells are still more sluggish and become passively stretched in the longitudinal direction as the surface increases in extent by active growth and division of the outer cells (Fig. 33, A). At the same time the middle layers of their walls become mucilaginous and separate the bodies, central cells from one another laterally, thus giving rise to the characteristic structure of the medulla. ${ }^{\mathrm{I}}$

This same contrast between outer and inner cells can also be seen in other Brown Seaweeds (not at all closely allied to Fucus) whose thallus consists of stout

FIG. 35-A, developing oogonia from surface of conceptacle with accompanying hairs. B, body cell of oogonium containing eight young eggs (female gametes). $\mathrm{C}$, freeing of the eight eggs by bursting of the bladder surrounding them. D, single egg (female gamete) surrounded by swarming male gametes. E, fertilisation. The nucleus of a sperm (male gamete) (sp.n.) has penetrated the egg and is approaching its nucleus. F, sperm nucleus in contact with wall of egg nucleus. G, sperm nucleus inside egg nucleus.

I The hypha-like chains of thick-walled cells (fibres) which form the whole of the holdfast, the greatest part of the medulla of the stipe, and also appear in the midribs of the fronds, begin to grow out from the cortical and the medullary cells as soon as these separate, and the "hyphæ" grow independently in the mucilaginous matrix. 
cylindrical threads from six to twenty cells thick. The surface cells are small and densely filled with phæoplasts, the central cells are larger and especially longer, and possess many fewer phæoplasts per unit volume (Fig. 33, C).

We can only relate this difference between surface and central cells, which is quite a general feature of bulky algæ, to the much more favourable conditions for active growth and division in which the surface cells are placed. They get more oxygen and more dissolved salts, for all these are obtained directly from the surrounding water; they are also better illuminated. Thus it appears that the differentiation of tissues in these algæ depends directly on the different conditions under which the cells develop. The different functions of the tissues in the adult alga are determined by their structure and position in relation to the source of food and oxygen and to the growth of the thallus as a whole. Since growth is localised at the tips of the branches, the medullary cells, as explained on p. 220, owing to their structure and position, are the natural channels of conduction of the organic foodstuffs elaborated by the photosynthetic cells.

This is a case in which the origin of differentiation in organisms appears to be directly due to differences in the conditions in which different cells develop, i.e. to physical and chemical differences of environment. The differentiation so initiated is, if it results in a workable mechanism, fixed and further specialised in higher forms.

In the Laminariaceæ, the group of Brown Seaweeds which have the longest bodies, including the gigantic Macrocystis and also the big "tangles" 
(Laminaria) of our own coasts, some of the medullary cells are very highly specialised for conduction of organic substances, and are strikingly like, even in small details, the sieve tubes of the higher plants, described in Chapter XVI.

\section{PRACTICAL WORK.}

\section{Fucus.}

\section{Vegetative Structure}

(I) Make a sketch of samples of the thallus of Fucus, showing the holdfast, the stipe, the branching, the positions of the apical grooves, and the conceptacles. Note that the stipe is extremely tough and cannot be broken by pulling with the hands, whereas the frond can. If the frond is broken the hair-like fibres may be seen projecting from the broken surfaces.

(2) Examine a cross-section of the frond under the low power and draw a diagram ${ }^{x}$ of the general plan of distribution of the tissues, marking :-

(a) the palisade (photosynthetic) layer on the surface,

(b) the cortex of large isodiametric cells, with fewer phæoplasts per unit volume,

(c) the medulla of elongated cells running in strand in various directions and separated by a matrix of cell wall substance.

Note that the extra thickness of the midrib is caused by the greater thickness of the medulla in that region.

(3) Examine a cross-section of the frond under the high power, and make careful drawings ${ }^{2}$ of small samples of the various tissues, including the thick-walled fibres ("hyphæ "), which are mainly localised just below the cortex of the midrib, on the edge of the medulla. Note that the conducting cells run horizontally or obliquely in the wings, longitudinally (so that they are cut transversely) in the midrib.

(4) Examine two longitudinal sections of the frond (a) cut through the midrib at right angles to the surface of the frond, (b) cut parallel to the surface of the frond through the centre. Identify the various tissues already seen in transverse section, and from a comparison of the appearance of the cells in the 
two views deduce their shapes. Draw under the high power samples of the cells which are not identical in appearance in transverse and longitudinal sections. Note the thin transverse walls of the conducting cells.

(5) Examine transverse and longitudinal sections of the stipe, noting the differences between its structure and that of the frond, especially $(a)$ the worn surface with radial splits between the surface cells; (b) the absence of a distinct palisade layer and the radial rows of cortical cells with relatively thin tangential walls, indicating that this layer has been largely formed by secondary cell division parallel to the surface; $(c)$ the mass of fibres (" hyphæ") mostly cut transversely, forming the medulla, interspersed with large isolated cells (the original medullary cells). Make drawings to illustrate these points.

In the longitudinal section of the stipe identify the tissues seen in transverse section.

(6) Examine a longitudinal section through the apex of the frond, showing the apical cell and the origin of the adult tissues.

\section{Sexual Organs.}

(7) Examine a section across the reproductive region of the thallus, first under the low power. Note that the vegetative tissues have the same general characters as in the purely vegetative part of the frond, but that the medullary cells here form a network, the cells being cut in various directions : this is the result of the increase in thickness of this part of the frond, the strands of cells being drawn out in all, not only in one direction.

(8) Examine the development and structure of the antheridia and oogonia in the same or in different conceptacles, and draw as many stages of development as you can distinguish. Note also the "sterile" hairs in the conceptacle.

(9) Examine fresh material in which eggs and sperms have been liberated, and appear as little masses on the outside of the frond. In species with the sexes on separate plants the sperms can be distinguished by their bright orange colour. Mix some eggs and sperms in a drop of sea-water or in salt solution of about the same concentration. Draw under the high power an egg and some sperms and watch the movements of the latter. The early stages of fertilisation (conjugation) can often be seen. 


\section{THE SIMPLEST LAND PIANTS : LIVERWORTS} AND MOSSES. THE PTERIDOPHYTA.

Hitherto we have been dealing entirely (except in the case of the Fungi) with plants which live in water, and get the whole of the raw materials of their food supply from water and the substances dissolved in it. But very far back in the history of the earth some plants emerged from the water and established themselves on the land, henceforward getting their carbon dioxide and their oxygen for respiration direct from the air. These terrestrial plants have, in course of time, dominated the surface of the earth, and, like the terrestrial animals, have developed the most complex structures. The most highly developed group are the seed plants or flowering plants which are the most completely adapted to land life. The history of the evolution of the plant kingdom, beyond the stage of the earliest land plants, is mainly a history of increasing adjustment to terrestrial conditions. We know nothing of how plants succeeded in first emerging from the water, nor can we follow in detail the course of their subsequent evolution. But by studying the simplest land plants now existing, plants which have become stabilised, so to speak, at an early stage of adjustment to terrestrial conditions, we can 
get some idea of how these conditions affected the land migrants, and of the adjustments in structure and function that have taken place. It must be clearly understood, however, that the simplest forms of land plants now existing certainly do not represent the actual stages in the evolution of land vegetation. They represent rather side lines of the evolutionary tree which have become stabilised, and are probably incapable of much further evolution.

The plants known as Liverworts and Mosses are two groups of green plants, all comparatively small and with tissues of comparatively simple organisation. They resemble one another in the structure of their reproductive organs and in their life histories, but are distinctly different in the form and structure of the plant body. In the last respect the Mosses are decidedly more highly developed than the Liverworts. Both represent comparatively low stages in adaptation of the green plant to terrestrial life. Most of them live in situations where the soil and the air are constantly moist, so that they are in little danger of losing water by evaporation so quickly that they dry up. They are not nearly so completely protected as the seed plants against this risk by having an almost impermeable waterproof covering, though many of them are protected to some extent. And they can absorb water over much or over the whole of their surface, which the higher plants cannot do. Some species actually live in places (e.g. rock surfaces, walls, etc.) where they are liable to dry up, and these can remain alive, though dormant, in the dry condition; when wetted they quickly absorb water and resume active life.

Liverworts : Pellia.-This is a common liverwort 
growing on soil among damp herbage, or in marshes. Pellia has no differentiation into distinct stem and leaves, any more than Fucus has. The body consists of a flat green thallus, which may be elongated

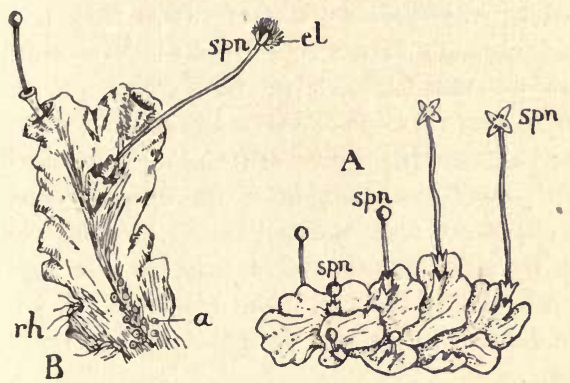

FIG. 36.-Pellia. A, plant (natural size) growing in bright light with crowded branches; spn., sporogonia of various ages, the oldest with long stalks and open capsules. B, part of thallus on a larger scale, showing forking at the tip; $r h$., rhizoids arising from lower side; $a$, antheridia; $s p n$., sporogonium with opened spore capsule showing bunch of hair-like elaters $(e l$.$) .$

and band-shaped, or may (especially in bright light) grow slowly in length and branch freely so that the plant is tufted (Fig. 36). The margins of the thallus (wings) are often "crisped" because they have grown quicker than the thicker central part, or midrib. The latter bears on its under surface brown rhizoids (Fig. 36, B, rh.), which enter the soil and absorb from it water and dissolved salts.

A thin cross-section of the thallus shows that all the cells are living and may contain chloroplasts, though these are mainly concentrated in the upper (sometimes also in the lower) surface layer. This represents an incipient differentiation of photosynthetic 
tissue, but there is little differentiation in the structure of the cells. In the surface cells starch grains may be detected enclosed in the chloroplasts if the plant has been exposed to fairly bright light; in the central cells there are large starch grains which have been formed by chloroplasts, out of which they have burst, and which may sometimes be still detected as green smears on the surfaces of the grains (cf. Pellionia, p. I26). The rhizoids are seen to be tubular outgrowths of the cells on the lower surface of the midrib. The midrib passes gradually into the wings, which at their outer edges are only one cell thick. Pellia, like Fucus, grows by division of apical cells at the tips of the branches of the thallus. The cells cut off from these divide further to form the whole of the tissue of the thallus.

Reproduction and Life History.-Pellia, like Fucus, and also like all the higher plants, is reproduced by means of sexually differentiated gametes; and as in Fucus these are formed in sexual organs arising from surface cells of the thallus. There is an important difference, however : in Pellia (as in all the Liverworts and Mosses and in the higher group called Pteridophyta) the sexual organ, both male and female, is covered in the mature state by a wall consisting of a layer of cells (Fig. 37, A, C, w), instead of by a cell wall only as in Fucus. This we may perhaps relate to the greater protection from evaporation required by subaerial life. The antheridia (Fig. 37, A) are spherical structures formed on the upper surface of the thallus, each in a little cavity due to the arrest of cell division in the thallus tissue just below the spot where the antheridium is formed and the growing out of the thallus cells to roof in the antheridium on each 
side. The sperms, very many of which are produced in each antheridium, are not unlike those of Volvox in shape, and like them have two flagella, but there is no trace of a chloroplast, and the long, narrow,
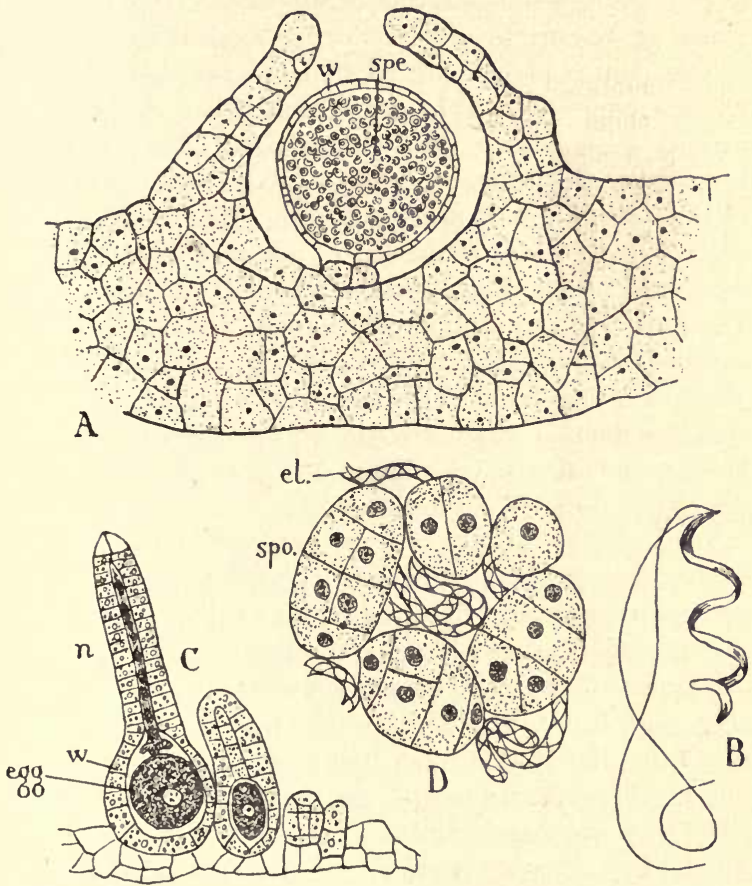

Fig. 37--Pellia. A, part of cross-section of thallus, showing ripe antheridium; $w$, wall of antheridium; spe., sperms (male) gametes coiled up. $\times 80 . \mathrm{B}$, single male gamete (after Guignard, $\times$ I22). C, four archegonia in various stages of development. On the left the archegonium is nearly ripe; $w$, wall of venter containing egg; $n$, neck, which will shortly open at the top. D, group of multicellular spores $(s p o$.) with spirally thickened elaters $(e l$.$) .$ 
spirally coiled body of the sperm consists almost wholly of nucleus, with a little colourless cytoplasm at the front end to which the flagella are attached (Fig. 37, B). The sperm cell is here reduced to its lowest limits-the paternal nucleus and the flagella, which are the means of carrying the nucleus to the egg.

The female organs are formed in groups, overhung by a membrane which grows out from the thallus behind them. They are flask-shaped, and in each there is a single egg contained in the body (venter $\mathrm{I}$ ) of the flask, which it practically fills (Fig. 37, C). The neck of the flask is composed of a single layer of cells continuous with those forming the wall of the venter. There is open communication from the egg to the exterior through the channel of the neck, which is filled with mucilage. This flask-shaped type of female organ, which is characteristic of the Liverworts and Mosses and also of the lower vascular plants (Pteridophyta), is called an archegonium.

When a film of water (rain or dew) is present on the surface of the thallus the ripe antheridium opens by the swelling and bursting of its wall and thus sets free the sperms, which are attracted to the mouth of the archegonium by the secretion of a substance, probably cane sugar, produced by the latter and diffusing out into the water. On reaching the mouth the sperms become entangled in the mucilage, wriggle down the neck and one of them fuses with the egg, which then becomes a zygote and secretes a wall.

The egg of Pellia (Fig. 37, C) is much smaller than that of Fucus, and this is related to the fact that it is not cast out from the thallus, and does not, therefore, have to depend on its own resources during germi- 
nation. The egg of Pellia is retained, after fertilisation, in the female organ, and germinates in situ, so that the embryo is protected by and can draw food from the thallus. This is the first example we have met with of the protection and feeding of the embryo by the mother organism, a condition which is of great importance, and is carried much further in the higher organisms, both plants and animals.

Sporogonium.-The embryo of Pellia, and indeed of all the Mosses and Liverworts, does not develop into a completely independent plant, but into a spore-producing structure called a sporogonium (Fig. 36, spn.), which remains attached to the thallus; and the spores produced by this germinate to produce new Pellia plants.

The mature sporogonium produced from the fertilised egg consists of (I) a foot embedded in and drawing organic food from the thallus, (2) a long stalk, and (3) a spherical head or spore capsule which is at first green. The stalk remains short for some two or three months in the spring while the spores develop, but when these are nearly ripe it quickly lengthens (Fig. 36, A), and in two or three days attains a length of 2 to 3 inches. The spore capsule is now dark, almost black, and in dry air it opens, four splits running down from the centre of its upper surface to the base where it joins the stalk. The four strips of wall thus separated fold back as four separate flaps, which form a cross-shaped structure standing out from the top of the stalk and exposing the mass of spores within (Fig. 36, A). If the opened capsule be examined with a hand lens there can be seen, interspersed with the dust-like spores, a number of delicate threads, the so-called elaters (Fig. 36, B, el.). These are long cells with spirally wound thickenings inside their walls 
(Fig. 37, D, el.). A large bunch of them arises from the base of the capsule. The elaters are very hygroscopic, i.e. very sensitive to small changes in the amount of water vapour in the air, and as they take up or lose water from the air they twist about, disturbing and scattering the mass of spores, which float off into the air.

The spores are large and multicellular (Fig. 37, D, $s p o$.$) , i.e. the original spore cell divides into several$ cells on ripening, and these contain chloroplasts. The spores do not form a resting stage in the life history, they die unless they quickly fall upon damp soil where they can germinate. One of the cells grows out and gives rise to the apical cell of the new Pellia plant, and the first rhizoids grow from the ends of the spore.

Besides the thalloid forms, of which Pellia is one, there are many Liverworts whose shoots have distinct stems and leaves, the leaf being a thin membranous structure consisting of a single layer of cells containing chloroplasts. The cells of the stem are longer and often thick-walled, but there is very little tissue differentiation. These leafy Liverworts live largely on rocks or tree-trunks in wet climates, and absorb water through the whole surface of the plant, especially the leaves. They are attached by rhizoids to the substratum on which they grow, but the rhizoids are of no importance in the absorption of water, as those of Pellia are.

Mosses.-The Mosses always have their shoots differ. entiated into stem and leaf, and most of them have a certain amount of tissue differentiation, considerably more than the Liverworts. They are on the whole larger plants than the Liverworts, and depend more upon absorption of water from the soil, though a 
number of species of Mosses are small plants growing on rocks and tree-trunks which can survive desiccation.

The rhizoids are branched multicellular cell threads which arise from the base of the stem and enter the soil, from which they absorb water. The stem has a central strand of long narrow thin-walled cells from which the protoplasm has disappeared, and these form a water channel from the absorbing region (rhizoids) to the evaporating region (leaves). The cells of the outer layers of the stem (cortex) have thick brown walls. The leaves ordinarily consist of a single layer of cells containing chloroplasts, but there is often a midrib, several cells thick, consisting of several cell-layers and possessing thin-walled waterconducting cells like those of the centre of the stem. The shoot grows by the division of a single apical cell at its tip, as in the Liverworts and in Fucus. Each segment cut off from this apical cell gives rise by division to the tissue of a single leaf and to the segment of the stem which bears it.

The Moss plant is a stage further on than the Liverwort in adaptation to land life. Though they can absorb water through their leaves, many Mosses have a regular water current from rhizoids to leaves. The existence of a definite water-conducting tissue corresponds with this localisation of absorption in one part of the body (rhizoids) and of evaporation in another (leaves), which is the mark of a terrestrial plant, and which is absent in Fucus and almost absent in Pellia. This feature is, as we shall see, carried to a much higher level of development in the Vascular Plants.

Sexual Organs and Sporogonium of Mosses.-The sexual organs and gametes of the Mosses have a general resemblance to those of Liverworts, though 
they differ in certain details of structure. The sexual organs, accompanied by hairs and surrounded by leaves, which are sometimes coloured yellow or red, are borne at the tips of upright shoots that have stopped growing.

The fertilised egg cell, like that of the Liverwort, germinates in situ within the archegonium, and the embryo grows into a sporogonium which, as in the Liverwort, remains attached to the parent plant. The Moss sporogonium, however, is of much more complicated structure than that of the Liverwort. It has a foot, stalk and spore capsule, but the stalk elongates much earlier and possesses a central water-conducting strand like that of the leafy stem. Surrounding this there is sometimes a layer of elongated living cells which conduct organic food to the developing spore capsule. These are comparable in function with the medullary cells of Fucus. The spore capsule itself is quite an elaborate structure. Its wall consists of several layers of cells containing chloroplasts and is covered by an epidermis with stomata, possessing in fact a structure like that of the leaf of a higher plant. Thus the sporogonium, which, during development, is supplied with organic food by the leafy parent plant, is able when nearly mature to make some of its own food from the carbon dioxide of the air and the water and salts brought up from the leafy parent plant through the foot and stalk. When the spores are ripe a lid is detached from the top of the capsule, and distribution of the spores is often assisted by the movements of hygroscopic teeth set round the edge of the opening, which thus perform the same function as the elaters of the Liverwort capsule. The spores germinate on damp soil to form an alga-like growth (proto- 
nema) of branching green cell threads, which spreads and often persists for a long time on damp soil. From the protonema leafy moss plants arise by budding.

Vegetative Reproduction.-Both Mosses and Liverworts spread largely by vegetative reproduction. The thalli of Liverworts branch freely as they creep on the soil, and the older parts, from which the branches have arisen, gradually die off, so that the branches, which have sent out rhizoids from their under surfaces, become detached independent plants. In the Mosses branching often takes place towards the base of the shoot, and the lateral branches, creeping on or in the soil, become attached by rhizoids, their tips growing up into the air. The decay of the older part of the shoot makes the new shoots independent plants. Very many Mosses branch at the base in this way, and the aerial shoots so produced grow up in close neighbourhood, their tips forming the surface of the characteristic moss tufts or cushions.

\section{Vascular Plants (Pteridophytes and Seed Plants).-} The plants above the Mosses in the scale of adaptation to terrestrial life are marked by several great steps in advance. First they have true roots instead of rhizoids. These are branches of the plant body many cells thick which enter the soil, branch and form very efficient organs for fixing the plant and absorbing water and salts from the soil. The branches of the root are comparable with the branches of the shoot, and like them consist of complicated tissue structures, but they differ from them in several important respects. We shall have to consider these differences in detail in later chapters. Here we need only note that roots are colourless, and that they often bear root hairs, which are tubular outgrowths of the surface cells 
like the rhizoids of Pellia, increasing the absorptive surface.

Secondly, there is a highly differentiated double conducting tissue system-the vascular system-running throughout the plant, and consisting of a waterconducting and an organic food-conducting portion. We have already seen that something of the kind, though not very highly differentiated, exists in the stalk of the sporogonium in Mosses. This double conducting system is a necessity in bulky plants in which the regions of water absorption, evaporation, photosynthesis and growth are all localised.

Thirdly, the photosynthetic organs, the foliage leaves, are typically plates of tissue several cells thick, in which the photosynthetic tissue (mesophyll) is interpenetrated by intercellular air spaces and is covered externally-in common with the whole shoot-by a layer of cells (epidermis) without chloroplasts, whose outer walls, in contact with the air, develop a waterproof layer, the cuticle. The epidermis and cuticle are pierced by pores (stomata), each surrounded by a pair of special cells (guard cells) containing chloroplasts which regulate the size of the pore (Figs. 50, 5I).

Most of these characters are foreshadowed in the Mosses. In the sporogonial wall, for instance, we met with the essential characters of the mesophyll, epidermis and stomata of the foliage leaf; and a cuticle is developed. more or less strongly both on the outer surface of the sporogonium and elsewhere. But the cuticle is neither so universal nor (usually) so strongly developed in Mosses and Liverworts as in the Vascular Plants. Again, we saw that rudiments of the double conducting system are found in the stalk of the sporogonium, which is in all respects the 
structure most highly adapted to subaerial life below the level of the Vascular Plants themselves. And finally, in one of the highest families of Mosses we have underground shoots which have many of the characters of true roots. But in their totality the features described above are characteristic of andapart from a few aberrant forms adapted to special conditions of life-are universally found only in the Vascular Plants, which include the Pteridophytes and the Seed Plants.

The Pteridophyta (Ferns, Clubmosses and Horsetails).-Important as these plants are to the botanist, it is beyond the scope of this book to devote to them any special study. All the vegetative characters of the Vascular Plants can for our purposes be more conveniently considered in connexion with the Seed Plants. But some knowledge of the outline of the life histories of Pteridophytes is essential to understanding the later phases of adaptation to terrestrial life which are characteristic of the Seed Plants.

In the Mosses and Liverworts we have two kinds of reproduction which regularly alternate with one another in the life history-the sexual process of fertilisation, which can only be carried out in water owing to the, fact that the sperms are motile swimming cells, and the process of spore formation and dispersal, which takes place in air. This emphasises the incompleteness of adjustment of these plants to terrestrial (subaerial) life. In the Pteridophyta, which include three or more divergent stocks or phyla of plants originating so far back in early geological time (certainly before the Devonian rocks were laid down) that we know nothing of their actual origin, we still find these two alternating types of reproduction, in spite of the 
development of the structural characters of the vegetative body described in the last section, which adapt them for terrestrial life.

In the Ferns we find a thalloid structure called the prothallus (Fig. 38, A), resembling in a general way the thallus of Pellia, which can only live in damp places, which, like Pellia, forms rhizoids $(r h$.$) on its lower$ surface, and which bears sexual organs (Fig. 38, A, an, $a, \mathrm{~B}$ and $\mathrm{D}$ ) very much like those of Pellia (but only on its lower surface). The sperms are formed and liberated in much the same way, though they differ in having numerous flagella (Fig. $38, \mathrm{C}$ ) instead of only two, and fertilisation takes place in a film of water on the surface of the prothallus. The fertilised egg (zygote) germinates in situ, producing an embryo within the archegonium wall. The embryo soon sends a sucker (the foot) into the tissue of the prothallus (Fig. 38, E). But now comes a wide divergence. Instead of developing into a sporogonium which remains attached to the thallus during its whole life, the embryo of the Fern soon develops a root which penetrates the soil, and a leaf which rises above the soil and begins to carry on photosynthesis (Fig. $38, F, r$ and $l$ ). The development of the stem is slow, but a second larger leaf and a second larger root are shortly produced. During this time the young plant has been drawing food from the prothallus, but after a while it becomes quite independent (the prothallus ultimately dying off), and grows constantly bigger, successive

Fig. 38.-Life history of Fern. A, prothallus from below; $r h$., rhizoids; an., antheridia; $a$, archegonia. $\mathrm{B}$, antheridium containing coiled sperms. C, single sperm with numerous flagella. $\mathrm{D}$, archegonium; $e$, egg; $n$, neck. E, section through embryo drawn in outline, showing prothallus, foot, beginnings of first root, first leaf, and position of growing point of stem, not yet developed; $r h$., rhizoids of prothallus. F, young plant still 

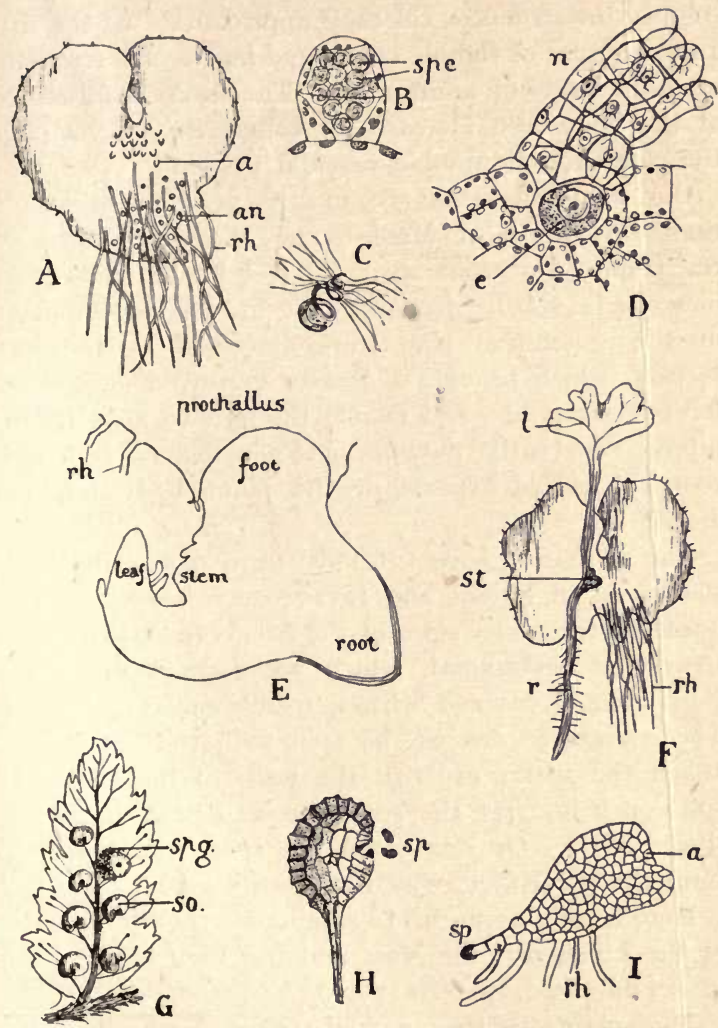

attached to prothallus with well-developed first leaf and root; st., stem (still small). G, pinnule of fern leaf from below showing seven sori (so.) and ripe sporangia (spg.) protruding from below membrane of sorus. $H$, single sporangium opening to set free spores (sp.). I, spore (sp.) germinated to form prothallus; $r h$., rhizoids; $a$, apical cell. 
leaves becoming larger and larger, and more and more complex in structure till they approximate to the size and structure of the big compound leaves characteristic of most kinds of adult ferns. The internal structure of the body also increases in complexity, developing the characteristic double vascular system.

Our common ferns are mostly very much larger than Liverworts or Mosses, and the Tree Ferns of the tropics are often 20 or 30 feet high. This great increase in stature is dependent on the development of the vegetative characters described in the last section, which provide a greatly improved equipment for terrestrial life, and enable the fern to grow indefinitely, constantly putting out new leaves and new roots. The older parts of the plant body tend to die off.

The adult fern leaves (fronds) form spores similar to the spores of Mosses and Liverworts. These are produced on the under sides of the fronds in little bag-like structures (sporangia), which are formed in groups (sori) usually covered with a membrane (Fig. 38, G). The sporangia can just be seen with the naked eye. When the spores are ripe the walls of the sporangia split open and set the spores free to float in the air (Fig. 38, H). On damp soil the spore germinates to form the prothallus, which bears the sexual organs.

Here it may be useful to summarise the life histories of the Liverwort, the Moss and the Fern in the form of a table (see opposite page).

The plant bearing sexual organs is called the gametophyte, the spore-bearing generation (free living only in the case of the Ferns and other Pteridophytes) the sporophyte. In the Moss the gametophyte is more highly adapted to terrestrial life than 
LIFE HISTORIES OF LIVERWORT, MOSS AND FERN 247

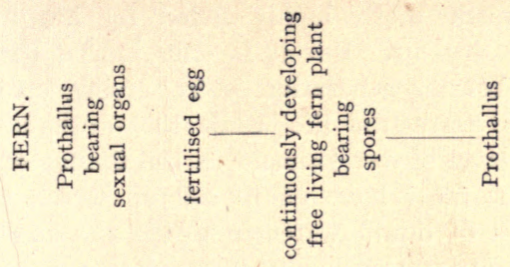

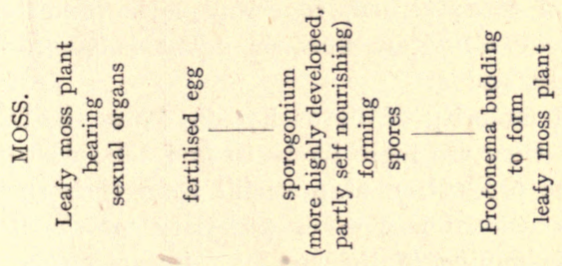

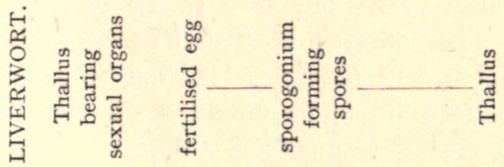

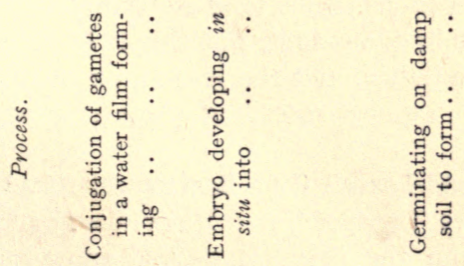


in the other two, and the sporogonium of the Moss is also much more highly fitted for life in comparatively dry air. But it is only in the Fern that the spore-bearing generation is free living, completely adapted to terrestrial life, while the prothallus is on the same level of development as the thallus of Pellia, and like it must have damp soil on which to grow and a film of liquid water in which the sperms can swim for fertilisation. The Fern, therefore, though a terrestrial plant, is still tied, so far as its sexual reproduction is concerned, to semiaquatic conditions, undoubtedly a heritage from the aquatic algæ from which it is descended.

The majority of Ferns live in damp shady places, where they are more likely to find suitable conditions for the production of prothalli from their spores and the occasional free water necessary for the liberation and swimming of the sperms. But some depend for their propagation almost entirely on vegetative reproduction, and these can live in comparatively dry habitats. The common bracken fern (Pteridium aquilinum) is a good example. It flourishes on rather dry sandy soil, its underground stems spreading far and wide and continually sending up new fronds, while the older parts gradually die off. It can and does produce prothalli from its spores when they fall in a suitable damp spot, but this is in many localities a rare possibility, and the great bulk of this vigorous, aggressive, rapidly spreading plant is produced vegetatively.

The Small-Leaved Cone-Bearing Pteridophytes.-The Clubmosses (Lycopods) and Horsetails (Equiseta) differ widely from the Ferns in general appearance, though the broad outlines of their life history are essentially 
the same. Instead of large compound fronds they have small scale-like leaves, and in the Horsetails these are so small that the great bulk of the photosynthesis is done by the surface tissues of the stem. Another point in which they differ from the Ferns is that their sporangia are borne in cones, i.e. in connexion with small crowded leaves, mostly differing more or less from foliage leaves, at the ends of some of the shoots (Fig. 39, A). The prothalli also are unlike those of Ferns, being cylindrical or thin, branched structures instead of flat. In many of the Lycopods the prothalli are not green, but live saprophytically in humus or humous soil. These prothalli grow very slowly, and the sporophytes depend very largely on vegetative reproduction. The British species of Lycopods live mostly on the hills of the north and west where the soil is humous, i.e. contains a large amount of plant débris, which decays very slowly indeed, owing to the cool damp climate. The Horsetails mostly live in marshes, by pond sides and in damp places generally, but one species, the Field Horsetail (Equisetum arvense), grows very commonly on hedgebanks and the edges of fields, though on stiff, moisture-retaining soils. It is the only British Pteridophyte occurring in such situations and holding its own with the dominant seed plants-the common hedgerow plants. Like the other Horsetails, it has long underground stems which spread below the soil and send up the green aerial shoots at intervals.

Heterospory.-In the great majority of existing Pteridophytes the spores are all alike, but in a few two kinds of spores are produced, large megaspores, much larger than ordinary spores, only one or four being produced from a single sporangium, and 
small microspores, about the same size as ordinary spores (Fig. 39, C). These are formed in separate sporangia (Fig. 39, B). In these heterosporous species the ordinary kind of free-living prothallus, bearing both male and female sexual organs, is not produced. The megaspore on germination produces a small mass of cells at its apex, the bottom of the spore being sometimes afterwards filled with cells (Fig. 39, G), and on this small prothallus one or more archegonia are formed. The microspore produces a prothallus of even fewer cells, in most cases inside the microspore wall (Fig. 39, D), and with the exception of one or two, the whole of these form a single antheridium (Fig. 39, D). The spore absorbs water and the wall bursts, setting free the sperms $(E, F)$. Fertilisation takes place as in the ordinary (homosporous) Pteridophytes. The fertilised egg obtains the food which enables it to grow into a self-supporting plant mainly or entirely from the organic food supplies stored in the megaspore $(G)$.

The far-reaching reduction of the prothallus and the suppression of its powers of independent growth and nutrition that we see in these heterosporous Pteridophytes involves the direct dependence of the young sporophyte produced from the fertilised egg not only on the gametophyte but on the spore (megaspore) which produced it, instead of on a free-living sexual generation, as in the homosporous Pteridophyte. This opens the door, so to speak, to a further adaptation to land life, in which the megaspore is retained in the sporangium, and the sexual generation need no longer

FIg. 39.-Selaginella, a heterosporous cone-bearing Pteridophyte. $A$, end of leafy branch, with three cones (natural size). B, portion of stem of cone with megasporangium (left) opened showing megaspores, and (right) microsporangium setting free microspores. $\mathrm{C}$, microspore (mi.) and megaspore (me.) showing thick wall 

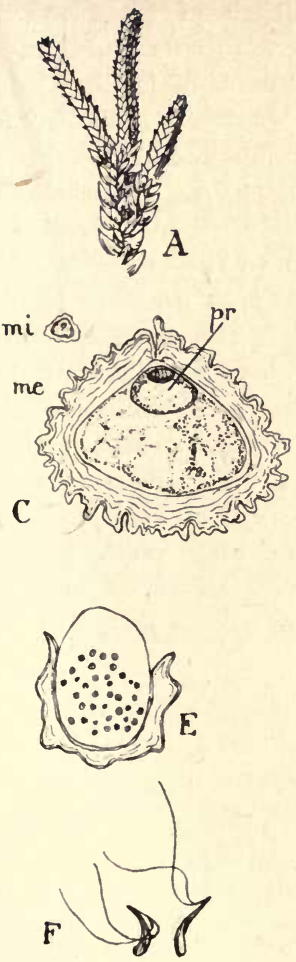
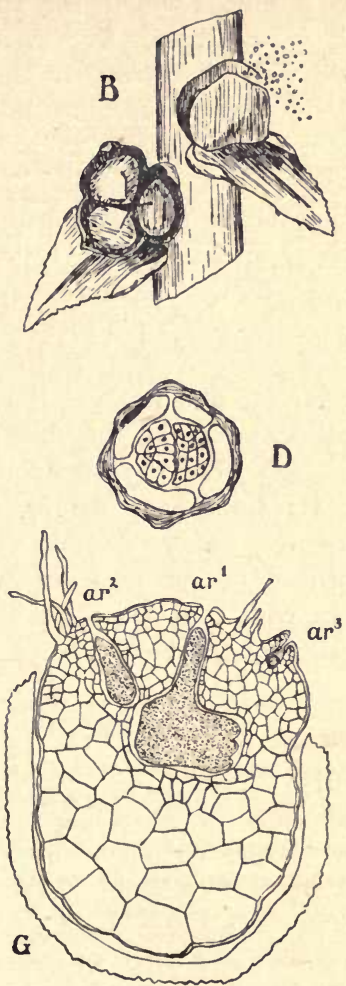

and cell inside $(p r$.$) from which the prothallus will be formed. D,$ microspore germinated to form antheridium which completely fills the spore cavity. E, microspore opened with sperms embedded in mucilage formed from breakdown of walls in water. F, two free flagellated sperms. G, megaspore opened and germinated to form prothallus with three archegonia (ar.), the eggs in two of which have developed into embryos at the expense of the prothallial tissue, while the third (ar3) is unfertilised and still contains the egg. 
be produced under the conditions of moist soil and air where it can live as a semiaquatic and find the free water necessary to the process of fertilisation by motile free swimming male gametes. The freedom from this necessity is not however attained by the existing heterosporous Pteridophytes, in which the microspores still germinate only on damp soil and the sperms still have to swim to the archegonia in a water film. The necessary step to complete freedom from the semiaquatic habitat is the retention of the megaspore in the megasporangium, where it may germinate and produce the eggs in a sheltered position, withdrawn from the danger of desiccation, and the bringing of the microspores to the megasporangium or its immediate neighbourhood, so that the male gametes produced from them may reach the egg without the presence of external water. This is the step which, as we shall see in the sequel, has been taken by the Seed Plants.

\section{PRACTICAL WORK.}

(I) Examine plants of Pellia, if possible growing plants. Sketch the form of the band-shaped or crisped green branching thallus, with thick midrib attached to the soil by rhizoids and passing gradually into the thinner wings at the edges.

(2) Examine a transverse section of the fresh thallus, showing the uniform thin-walled tissue. Note that the chloroplasts are mostly concentrated in the upper (sometimes also in the lower) surface layer of cells. In these small starch grains can be seen if the plant has been well illuminated. The central cells contain large starch grains on the surface of which can sometimes be seen the remains of a chloroplast. From the lower surface of the midrib rhizoids arise, each as a tubular branch of a single cell.

Draw under the high power samples of the cells of the upper and lower surfaces with rhizoids and of the central cells.

3) Place some of the contents of the ripe spore capsule of Pellia in a drop of dilute glycerine and examine the spores and 
elaters under the high power. Note that each spore is divided into several cells packed with chloroplasts.

(4) Examine a prepared longitudinal section through the centre of a spore capsule showing stalk, capsule wall, spores and elaters.

(5) Make a sketch of a single plant of Funaria (or other moss) with the naked eye or under a hand lens. In Funaria note the short axis (stem) with a tuft of brown rhizoids at its base, and bearing broad delicate green leaves. The leafy axis may bear a slender stalk terminating in a nodding pear-shaped spore capsule.

(6) Detach a single leaf, mount in a drop of water and examine under the high power. Observe that the leaf consists of a single layer of cells more or less alike, except the midrib, which is composed of elongated cells. Note the leaf cells have thin walls : each is lined with a layer of cytoplasm containing chloroplast and enclosing a large central vacuole.

(7) Examine some of the brown rhizoids in dilute glycerine, and note that they are branching threads of different thicknesses composed of long cylindrical cells with oblique end walls.

(8) Examine a prepared transverse section of a moss stem showing the outer cortex of thick-walled cells, the inner cortex of thin-walled cells, and the strand of narrow thin-walled waterconducting cells in the centre. 


\section{CHAPTER XV}

\section{THE SEED PLANTS: FORMS AND LIFE HISTORIES}

TAKEN as a whole the Seed Plants represent the most complete adaptation to terrestrial life that we find within the plant kingdom; and, correspondingly, they are the plants which now dominate the face of the earth. On the vegetative side this adaptation is represented by the broad features of organ and tissue development described on pp. $24 \mathrm{I}-2$, which are common to all the vascular plants, but are carried to a higher pitch of specialisation in the Seed Plants than in the Pteridophyta. On the reproductive side it is represented primarily by the seed itself and by the structures and processes leading up to and accompanying its development. The seed is a direct metamorphosis of the ovule: in other words the ovule changes directly into the seed. And the ovule is simply a megasporangium containing a single megaspore, which has germinated, not by producing any structure external to itself, but by producing the female gamete or egg inside the megaspore as a result of the division of the megaspore nucleus. The male gamete, produced, as in the heterosporous Pteridophytes, inside the microspore, is brought to the neighbourhood of the megasporangium still contained in the microspore, and then, by the growth of a germ tube put out from the microspore, to the megaspore and to the egg itself. The process 
of conjugation of the gametes is thus rendered quite independent of external water, and the gametes are withdrawn from the danger of desiccation. The fertilised egg also germinates inside the megaspore, where it produces the embryo, which develops up to a certain point and then enters upon a resting stage. The ovule (megasporangium) containing the resting embryo, together with a store of food providing for the development of the embryo into the free-living plant, is called the seed. Here then not only the sexual generation, but also the early stages of the new individual developed from the fertilised egg are produced within the body and nourished at the expense of the parent sporophyte.

The hundreds of thousands of existing species of Seed Plants are exceedingly various in form and life history. We can only consider a few of the general types into which these can be grouped.

First, a broad distinction, though by no means an absolute distinction, can be drawn between woody plants and herbaceous plants. The subaerial shoots of woody plants (trees and shrubs) persist from year to year, and constantly form new portions of the shoot system which are continuations of the portions already formed. At the same time the parts already formed in most cases grow continuously in thickness, and the new layers so added are mainly composed of hard woody tissue. Thus the whole plant body constantly increases in size.

In the herbaceous plant, on the other hand, the subaerial shoot consists mainly of soft tissue and is short-lived, generally dying down after one growing season. The whole vegetative plant may die after one season's growth, when its seeds have ripened 
(annual plants), or the aerial shoots alone may die off, leaving persistent underground shoots (perennial plants). The underground shoot may be woody and persist indefinitely, constantly increasing in thickness as well as in length like a woody subaerial shoot, or while growing at the apex from year to year it may continuously die off behind. In this chapter we shall consider some of the leading herbaceous types of seed plant body.

Erect Herbaceous Annual.-This is the simplest type of herbaceous plant, but is not to be considered the most primitive, the form from which the other types are derived. On the contrary, it is believed by botanists, on very good grounds, that woody perennial plants are the most primitive forms of the higher plants, and that the herbaceous, and finally the annual plants, have been derived from these.

The body of an annual (Fig. 40, A) consists of a descending portion, the primary root or taproot, and its branches. These penetrate the soil, fix the plant, and absorb water and inorganic salts from the soil; and the green shoot, which ascends into the air and consists of the axes (stems) and typically flat plate-like organs borne on them-the foliage leaves.

The first leaves borne by the stem-those nearest the root-are typically a pair already formed on the embryo in the seed, and generally simple in form: these are called the cotyledons. The part of the stem between the cotyledons and the top of the root is called the hypocotyl. In the young seedling plant

FIG. 40.-A, diagram of annual plant: t.r., taproot; hyp., hypocotyl ; cot., cotyledouns; $l$, foliage leaf; $a x$., axillary bud; $t . b$., terminal bud ; fl.b., flower bud. B, part of underground stem (rhizome) of Coral root (Dentaria) showing fleshy scale leaves (sc.l.) of adventitious roots $(v)$, lateral bud (l.b.), aerial shoot $(a . s$.$) , arisen from the ter-$ 


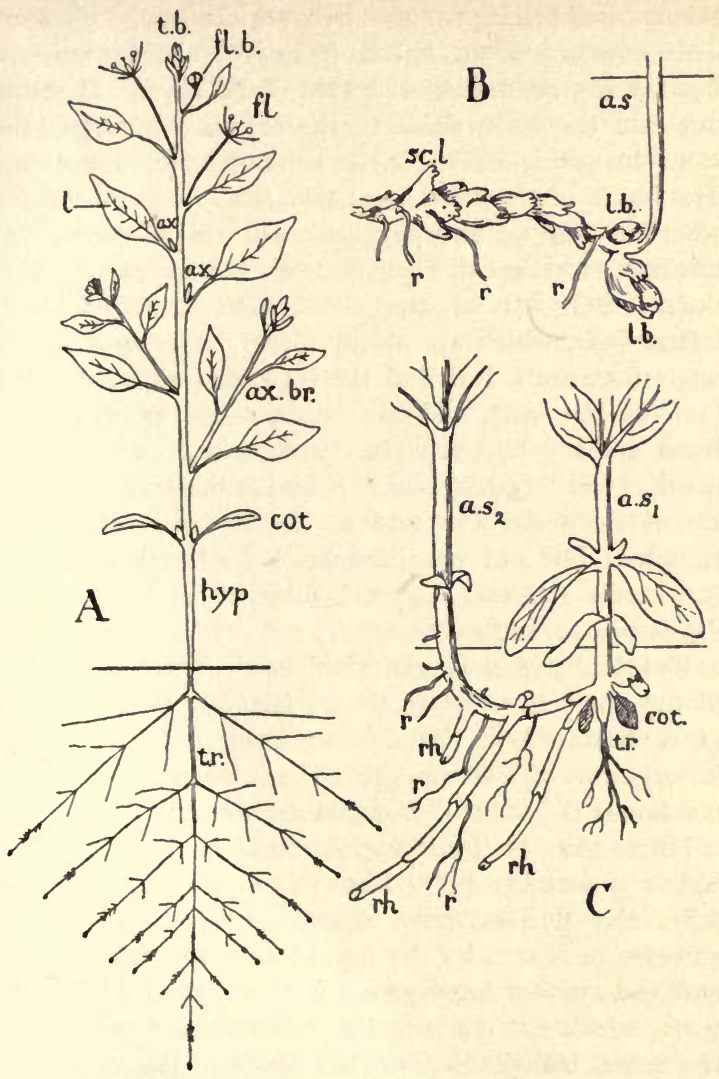

minal bud. The rhizome is branching from two lateral buds. C, base of a young plant of the Yellow Loosestrife (Lysimachia vulgaris), with lateral shoots forming rhizomes. cot, cotyledon; t.r., taproot; $a . s_{x}$, aerial shoot from epicotyl; $a . s_{2}$, from axil of cotyledon; rh., rhizomes. 
the terminal bud is produced between the two cotyledons. This consists of an apical (primary) shoot meristem bearing the rudiments of leaves on its sides. It grows out into the main shoot of the seedling (epicotyl), the stem elongating and the leaves developing and unfolding. The levels of the stem from which a leaf (or a pair or whorl of leaves) actually arise are called nodes, and the bare stretches of stem between the nodes are internodes. Branches of the shoot are developed from lateral buds, which are nearly always developed in the angle between a leaf and the internode above the leaf (axil of the leaf), and are hence called axillary buds. Each axillary bud repeats the structure and development of the terminal bud. A bud is maintained at the tip of the main axis and at the tip of each branch (terminal buds of the branches), the meristem being constantly renewed by cell division as its products develop into mature tissue.

Eventually flowers are produced. These are parts of the shoot or separate shoots (i.e. the whole product of a lateral bud) bearing specialised leaves (floral leaves), some of which produce the gametes. Sometimes one flower is produced from the terminal bud, generally a larger number from various axillary buds. A bud which is forming floral leaves is called a flower bud. After the flowers have opened, conjugation of the gametes produced by the floral leaves has taken place, and the zygotes have grown into embryos within the seeds, which are contained in the modified remains of the flower called the fruit, the whole of the vegetative part of an annual plant-the entire root and shootdies, the life of the species being continued solely in the seeds, which under favourable conditions in due course germinate to form new plants. 
Rhizome-forming Plants.-Many herbaceous plants, indeed the great majority of species, do not merely form a single aerial shoot system which dies at the end of the growing season, but, in addition to aerial shoots, an underground or surface shoot or shoot system which survives from one season to the next, and is called a rhizome. The rhizome may be formed by the whole epicotyl of the seedling plunging into the earth, and growing horizontally instead of vertically upwards to form an aerial shoot; but in most cases the rhizome is produced by an axillary bud near the base of the aerial shoot growing out horizontally. Usually the rhizome bears scale leaves (Figs. 40, B, 4I, A, C, sc.l.) which remain small and do not turn green, but sometimes it bears foliage leaves which grow up into the air on long stalks. From the rhizome one or more aerial shoots, usually bearing both foliage leaves and flower buds, grow up into the air. Very often the terminal bud of the rhizome turns up and produces a single aerial shoot (Figs. 40, B, 4I, B, a.s.), the growth of the rhizome itself being continued by the growth of one or more axillary buds. Aerial shoots also often arise directly from axillary buds.

At the end of the growing season the aerial shoots with their foliage leaves die down, but the rhizome remains alive in a dormant condition till the beginning of the next growing season, when growth is renewed from terminal and lateral buds and a fresh portion of the rhizome and new aerial shoots are produced. The older parts of rhizomes commonly die off and decay. In countries like England, with comparatively mild winters, the growth of the rhizome of many species continues throughout the winter except during periods of severe frost, and new leaves and buds are slowly produced, 


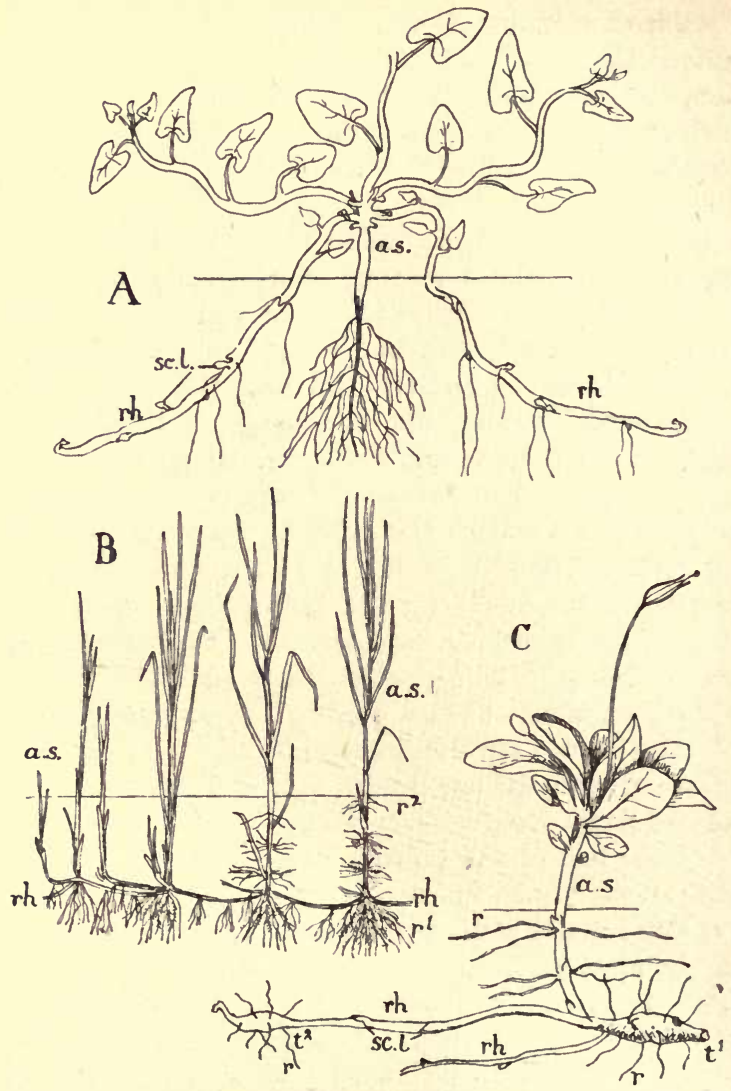

FrG. 41.-A, young plant of the Field Bindweed (Convolvulus arvensis), showing the thin white underground shoots $(v h$.) which ramify deeply in the soil and make the plant a troublesome weed. B, horizontally growing rhizome of a grass. C, plant of the Chickweed Wintergreen (Trientalis) which has arisen from the tuber ( $\left.t^{2}\right)$. The new tuber $\left(t^{2}\right)$ has arisen at the end of the rhizome. rh., rhizomes; $r$, adventitious roots; sc.l., scale leaves; a.s., aerial shoots. (After Warming.) 
in preparation for the more active growth and the formation of the aerial shoots in the next spring.

Roots are always produced on rhizomes, either generally distributed on the surface or localized at the bases of the aerial shoots (Fig. 4I, B), or at the bases of foliage leaves arising directly from the rhizome. Such roots, arising directly from a stem, are sometimes called adventitious roots as opposed to the taproot and its branches, which in rhizome-growing plants are shortlived. Organic food materials (starch or oil-sometimes sugar-and proteins) are stored in rhizomes during the winter, and are " mobilised," i.e. converted into soluble forms when new growth takes place.

Rhizomes may be short and compact, the axis of the rhizome shoot being then usually vertical or oblique and growth being very slow (" male fern," dandelion, plantain); or they may be elongated and horizontal, often running for considerable distances below the surface of the soil (Figs. 40, B, 4I). It is this type of rhizome, with brown or dingy coloured surface, from which the name (meaning "root-like organ") is taken. But rhizomes are quite sharply distinguishable from roots because they bear leaf structures and have the anatomical construction of stems, not of roots. The spread of many plants, such as sedges, rushes, some grasses (e.g. couchgrass or "twitch"), is due to this type of rhizome, which grows and branches freely, sending up aerial shoots from the upward turning terminal, or from lateral buds. Stolons or runners are very thin, horizontal, quickly growing shoots, which run usually upon the surface of the soil (e.g. strawberry runners), and do not bear roots. The terminal bud eventually turns up, thickens, produces a rosette of foliage leaves, strikes roots down into the soil, 
and becomes an independent plant. These offsets are regularly used by gardeners to propagate the plant.

Some horizontal rhizomes, on the other hand, are thick, fleshy and comparatively slow growing (Solomon's Seal), and these store considerable quantities of organic food reserves during the winter.

Tubers.-When the food is localised in a definite portion of an underground stem, the rest being thin, the swollen portion is called a tuber (Fig. 4I, C, $t$ ). The beginnings of tuber formation are well seen in the Chinese Artichoke (Stachys tuberifera). Many species of Stachys have uniform rhizomes, but in the Chinese Artichoke several successive internodes are swollen, the nodes being somewhat constricted by comparison with the internodes and bearing alternating pairs of triangular scales. A thinner portion of the rhizome follows, the terminal bud turning up to produce the aerial shoot. The lateral buds which continue the growth and branching of the rhizome grow out to form several thin internodes, followed by the thick tuberous portion in each case, so that the tubers are connected together in branching chains.

In the Potato plant (Solanum tuberosum) the main shoot is erect, but thin lateral shoots are formed from buds in the axils of basal leaves, and each of these grows horizontally or obliquely downwards and swells at its apex into a potato tuber. When very young (about the size of a pea) the tuber is seen to bear minute scale leaves, but these do not grow as the body of the tuber swells, and when the latter is full grown their scars appear, with the bud in the axil of each, as the " eyes" of the potato. The "rose" end of the potato is the apex of the tuber where the " eyes" are crowded. 
When fully grown the stalk separates from the tuber. If left in the ground over the winter, the eyes grow out next year into the aerial "haulms" of new potato plants. Roots ("adventitious roots ") are produced from the base of the haulm just above the point at which it springs from the tuber.

Corms.-This name is given to a local thickening at the base of the erect aerial shoot, which carries the life of the plant from one growing season to the next. At the end of the growing season the aerial shoot dies and falls off, leaving a scar on the top of the corm, which remains dormant during the autumn and winter, and produces new aerial shoots from axillary buds in the next growing season. The garden Crocus is an excellent example.

In the autumn, when the corm is "ripe" and ready for planting out, it is a rounded structure flattened at top and bottom and covered with thin brown membranous scales, which are inserted along horizontal lines encircling the corm and appearing as brown circular scars when the scales are stripped off. In a large vigorous corm there are two or three stout buds covered with white scales and projecting through the brown covering at the top of the corm. These can be seen to spring from the axils of the brown scales around the apical scar left by the detachment of the aerial shoot. Sometimes there are smaller buds on the sides of the corm in the axils of lower scales.

When the corm is planted, a circle of roots, the beginnings of which are already laid down in the ripe corm, first grow out, and growth soon begins in the axillary buds, which produce the green shoots that push up through the soil in February and flower in March. The rudiments of the foliage leaves and flowers 
can be clearly seen in longitudinal section of a bud taken in the autumn. The food which supplies the material for growth is stored, largely in the form of starch, in the tissues of the corm. When the aerial shoots are fully developed the base of each begins to swell by the growth in thickness of the stem tissue, and starch, made from the sugar produced by the foliage leaves, is deposited in this tissue. Thus a new corm is formed at the base of each aerial shoot, and each new corm goes on growing so long as the foliage leaves are alive (May or June). During the summer or autumn the new corms, covered by the scale leaves which were developed at the base of the aerial stem, and which gradually became dry and brown, become detached from the old corm, which is now finished and shrivelled, while the upper part of the aerial shoot, also dead, is detached from the top of the corm. Meanwhile axillary buds develop in the axils of the scale leaves of the new corms, swell and produce the rudiments of next year's foliage leaves and flowers, so that by October the new corms are "ripe."

If the Crocuses are left in the ground, the new corms develop where they are, and after a few years become very crowded, so that the new corms are apt to be small and feeble, often possessing only one new axillary bud which is strong enough to flower. That is why a better crop of flowers is produced if corms are taken up as soon as the leaves die, and only the most vigorous selected for replanting.

Other examples of plants which go through the winter in the form of corms are the Meadow Saffron (Golchicum autumnale)-found in meadows and woods especially in the west of England-which produces its new corm in the same way, but at the side of the old 
one; the bulbous buttercup (Ranunculus bulbosus); common in dry pastures; and Cyclamen.

Bulbs.-The life history of a plant which perennates by means of bulbs is very similar in general features to that of the corm-forming plants, the distinction being that in the bulb the store of food is contained in the scale leaves (bulb scales) of the winter shoot, the stem being represented by a disc at the base of the bulb. On this are inserted the outer membranous brown protective scales, and the massive bulb scales which form the greater part of the body of the bulb.

In the Tulip, which is a suitable type for study, there is however another difference. The aerial flowering shoot is produced from the terminal bud of the bulb, not, as in Crocus, from axillary buds. The new bulbs are formed, not by the swelling of part of the aerial shoot, but by the formation of buds in the axils of the bulb scales. These buds gradually swell during the growing season, as the old bulb scales become emptied, and thus produce the new bulbs inside the old one. It is even more important to dig up and remove tulip bulbs from the soil at the end of the growing season if the crop is to be maintained.

Some bulb-forming plants (onions, lilies, etc.) also produce under certain conditions small bulbs called bulbils in place of all or some of the flowers. These become detached, fall to the ground, and in suitable soil strike root and reproduce the plant.

Perennation, Multiplication and Spreading. - The types of more or less specialised underground shoots described-rhizomes, tubers, corms and bulbs-all serve to carry the plant on in the vegetative form from one growing season to the next, for they can exist unharmed in a dormant condition in the soil through the winter, 
when the aerial shoots have died; and they store food from which the next season's growth is started. Primarily these underground shoots are thus a means of perennation. Many plants which flower in early spring have bulbs, corms or rhizomes, and are able to make new growth quickly because of the great store of organic food at their immediate disposal.

When a rhizome branches and the old parts die off so that the branches are separated, the plant is propagated vegetatively, i.e. the number of individuals is multiplied by purely vegetative means, just as we saw happened in Mosses and Liverworts, and as happens also in Spirogyra when the cell thread, after increasing in length by cell division and growth, splits into lengths, and produces a corresponding number of new plants.

It is essentially the same process when a plant produces many tubers, or a Crocus corm several aerial shoots, at the base of each of which a new corm is formed, or when a bulb produces several new lateral bulbs. Though the plant is thus multiplied, the new individuals are developed close together, and compete for the same space, light and food-the species is not dispersed. But dispersal occurs, though gradually; if the underground stems spread widely, producing new roots and new aerial shoots from their tips. The advantage of the bulb or corm is that it is better protected from desiccation than many rhizomes, and is thus particularly well adapted to plants living in climates with a very dry season. South Africa, for instance, and the Mediterranean region have a large number of bulb and corm producing species. The British climate has no very dry season, and there are many more rhizomatous plants which are able to stand our comparatively mild winters perfectly well, 
though the rhizomes are not specially well protected, and often grow slowly throughout the winter.

The methods of vegetative propagation in the higher plants show that any shoot which has the power of rooting at its base may be regarded as a potential individual. In artificial propagation by means of cuttings, a branch shoot is cut off and the cut end stuck into damp soil. Roots are produced from this surface, and the detached shoot becomes a new plant. This extensive power of producing new individuals vegetatively is a character which separates the highest plants very sharply from the highest animals. The bodies of the latter are very highly integrated, i.e. they form very closely knit wholes, so that none of their parts can live independently, while the bodies of plants, with their indefinite growth and number of branches, constantly repeating the structure of the original shoot, and able under suitable conditions to live independently, do not form nearly such highly integrated individuals. This is largely connected with the fact that the bulk of their bodies is composed of comparatively undifferentiated living cells which are not so far removed from germ cells as those of the more highly specialised tissues of the higher animals. (Cf. pp. 206-7.)

\section{PRACTICAL WORK.}

\section{Annual Herbaceous Plant.}

(I) Examine a small herbaceous plant with a single main shoot and a taproot. Make a sketch of the plant showing the following parts : taproot, branch roots, hypocotyl, cotyledons, nodes, internodes, leaves, leaf veins, terminal buds, axillary buds, flowers or flowerbuds.

[Any small quickly developing annual is suitable if it retains its cotyledons till it produces flowers. Chickweed (Stellaria media) or one of the annual Veronicas- $V$. agrestis, Tournefortii or hedevefolia-does very well.] 


\section{Rhizomes.}

(2) Examine and sketch a portion of the Couchgrass or " Twitch" (Agropyrum repens) or any grass of similar habit. The main stem of the plant is the rhizome, bearing scale leaves and roots. The aerial leafy shoots arise from buds in the axils of the rhizome scales. Branching of the rhizome is secured by the outgrowth of other axillary buds which grow horizontally. Note the abundant production of roots at the bases of the aerial shoots, and the sharp points formed by the folded scales covering the terminal buds of the rhizome branches. These can penetrate stiff clay and often bore through objects such as potatoes which they may encounter.

(3) Compare the stout, more slowly growing rhizome of Solomon's Seal (Polygonatum). The scales which cover the terminal bud fall off, leaving only brown circular scale scars on the surface of the rhizome. The single aerial shoot is formed each year by the turning up of the terminal bud. The continuation and branching of the rhizome are secured by other buds formed in the spring. Compare the early spring condition with the summer condition (museum or herbarium specimens).

\section{Tubers.}

(4) Sketch the tuberously thickened branching rhizomes of the Chinese Artichoke (Stachys tuberifera). Note the thin portions of rhizome preceding and following the tubers, the constricted nodes, with triangular scales and axillary buds in opposite pairs, alternating on succeeding nodes, and the upwardly turned terminal bud.

(5) Examine potato tubers-a seed potato ready for planting, and a sprouted potato are most instructive. Mark on your drawings the scar of the tuber stalk, the eyes, the terminal bud, and in the sprouts the scale leaves, developing foliage leaves and adventitious roots. Examine a specimen showing early stages in the formation of tubers with the stalk arising from the axil of a leaf on the parent plant and the minute scales on the very young tuber.

\section{Corm.}

(6) Crocus, autumn stage. Carefully peel off the dry membranous scales and note that they are inserted one above the other, the lower ones overlapping the upper on the side of the corm, leaving brown circular scars when detached. Identify the stem scars at the top and bottom of the corm. The former is the scar of last spring's flowering and leafy shoot, of which 
the present corm is the swollen base. The scar at the bottom is the attachment of the present to last year's corm, which has shrivelled and fallen off. Note the circle of young roots ready to grow out round this scar.

With a sharp knife cut longitudinally through the corm, taking care to pass through the terminal scar and through one of the buds at the top of the corm, and note in the bud $(a)$ the external scale leaves, $(b)$ the rudiments of the foliage leaves, (c) one or more flower buds. Make a drawing of the cut face showing the vascular cylinders passing to the top scar and to the buds. Test the cut surface for starch.

(7) Compare the flowering stage of the Crocus plant, and note the swelling at the bases of the aerial shoots which will become the new corms.

\section{Bulb.}

(8) Tulip, autumn stage. Cut longitudinally exactly through the centre of the bulb. Note the disc-shaped stem at the base with point of attachment to the old bulb, rudiments of roots, membranous protective scales, fleshy bulb scales, terminal bud of aerial shoot with rudiments of foliage leaves and flower. Look for a small bud or buds in the axils of the bulb scales. Test the cut bulb scales with iodine.

(9) Examine the flowering stage and note the roots grown out from the edge of the bulb stem, and the flowering shoot developed from the summit of the bulb. Cut longitudinally through the bulb and note the flabby depleted bulb scales, and the new bulb or bulbs arisen from the axillary bud or buds seen in (8).

(10) Examine any other examples of underground shoots or of vegetative propagation that may be available. 


\section{THE TISSUE ELEMENTS OF SEED PLANTS}

BEFORE we study the microscopic structure of the vegetative organs of the Seed Plants it is desirable to have some knowledge of the different elements of which the tissues are built up, i.e. of the different kinds of more or less specialised cells of which the bodies of the higher plants are composed. If we can recognise the elements of the different tissues we meet with in the structure of root, stem or leaf we shall the more readily grasp the way in which these tissues are arranged and interrelated in these organs.

All the various elements that make up the tissues of a vascular plant are derived from embryonic cells such as are found in the primary meristems at the tips of the branches of the root and shoot (see Chapter VI, p. I02). Most of the body of a herbaceous plant, the "soft" tissues, are composed of living cells with cellulose wall of moderate thickness, and a large central vacuole, so that the cytoplasm forms a layer on the cell wall. The development of such cells from the embryonic cells was described in detail on p. I07. This sort of tissue is called parenchyma, and it forms most of the "ground tissue" of the plant body; it is also found associated in masses, strands or single cells with the more specialised tissues. The mesophyll of the leaf (pp. II5) is composed of this sort of cell in which the numerous plastids embedded in the cytoplasm have 
become chloroplasts. Certain adult living cells, however, differ in one way or another from this typical structure.
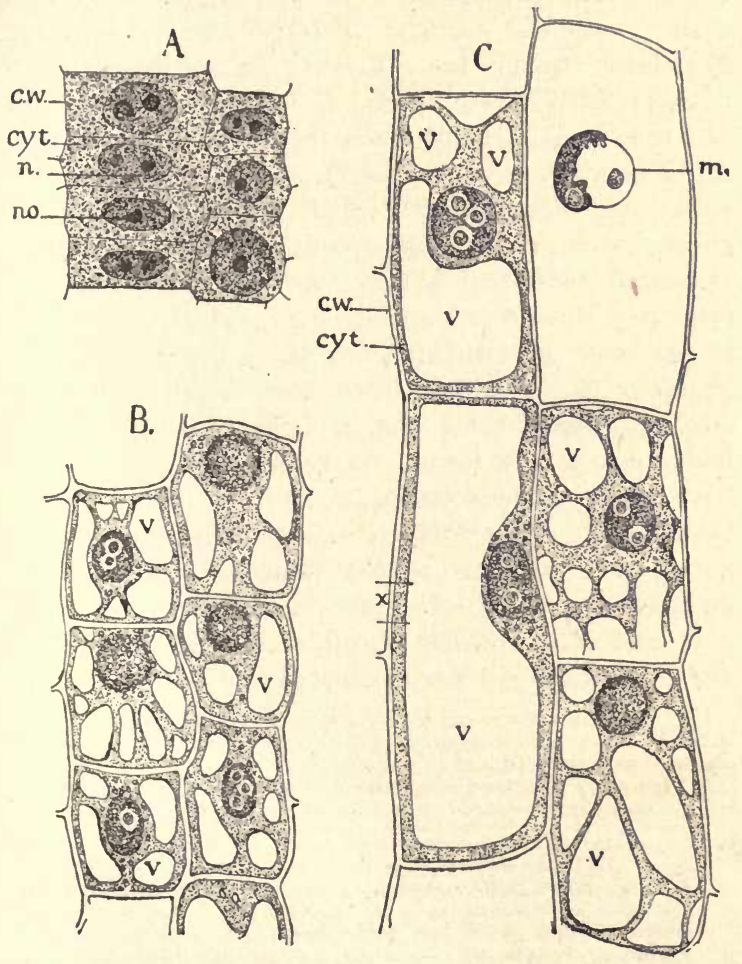

FIG. 42.-Development of adult living cells (parenchyma) from embryonic cells. A, embryonic (meristernatic) cells. B, beginning of vacuolation. C, fully grown cells (here much elongated) with characteristic large central vacuole; c.w., cell wall; cyt., cytoplasm; $n$, nucleus; no., nucleolus ; $v$, vacuole. 


\section{Cells rich in Protoplasm. Secretory Cells : Protein}

Cells.-Some cells remain densely filled with protoplasm even when adult, or at least contain much more cytoplasm in proportion to cell sap than the average tissue cell. The nucleus is large and conspicuous. Prominent among these are the cells (gland cells or secretory cells) whose special function is the secretion of a definite substance, such for instance as the cells of the nectaries of flowers which secrete a sugary fluidnectar; as also the cells which produce a particular enzyme, such as are found in seeds where large quantities of stored food material are brought into a soluble form in a short time, ${ }^{x}$ and diastase, cytase, or protease is produced in comparatively large quantities. The glands of insectivorous plants, also, secrete proteolytic enzymes which bring the proteins of their victims' bodies into soluble forms. Secretory cells, when active, have large conspicuous nuclei, and are often destitute of a vacuole. They closely resemble in structure and appearance the cells of the glandular epithelium of animals (Fig. 5, B) whose function is the same.

A kind of cell which resembles these in appearance and structure, and may indeed be said to belong to

It is to be understood that the production of such substances is by no means the monopoly of "secretory cells." They may be produced and are produced in the most various living cells. The cells called secretory are those which are specialised in this direction, and continuously produce large quantities of the substances in question.

FIG. 43.-Living tissue elements of the Seed Plant. A, secreting (glandular) hair arisen from epidermis of leaf. The four cells of the hair are richly protoplasmic and have conspicuous nuclei. Compare with living cells of epidermis and mesophyll below which contain much less protoplasm; s.h., secretory hair; $n$, nucleus ; $v$, vacuole ; ep., epidermis ; mes, mesophyll; ch.,chloroplast. $\mathrm{B}$, secreting cells from seed of Rye (Secale), with dense granular cytoplasm and conspicrous nucleus $(n)$. C, part of sieve tube in longitudinal section showing two sieve plates (covered with callose) and funnel-shaped slimy cell contents (contracted away from wall). On the right two companion cells (protein cells) with dense cytoplasm and conspicuous nuclei. On the left an 

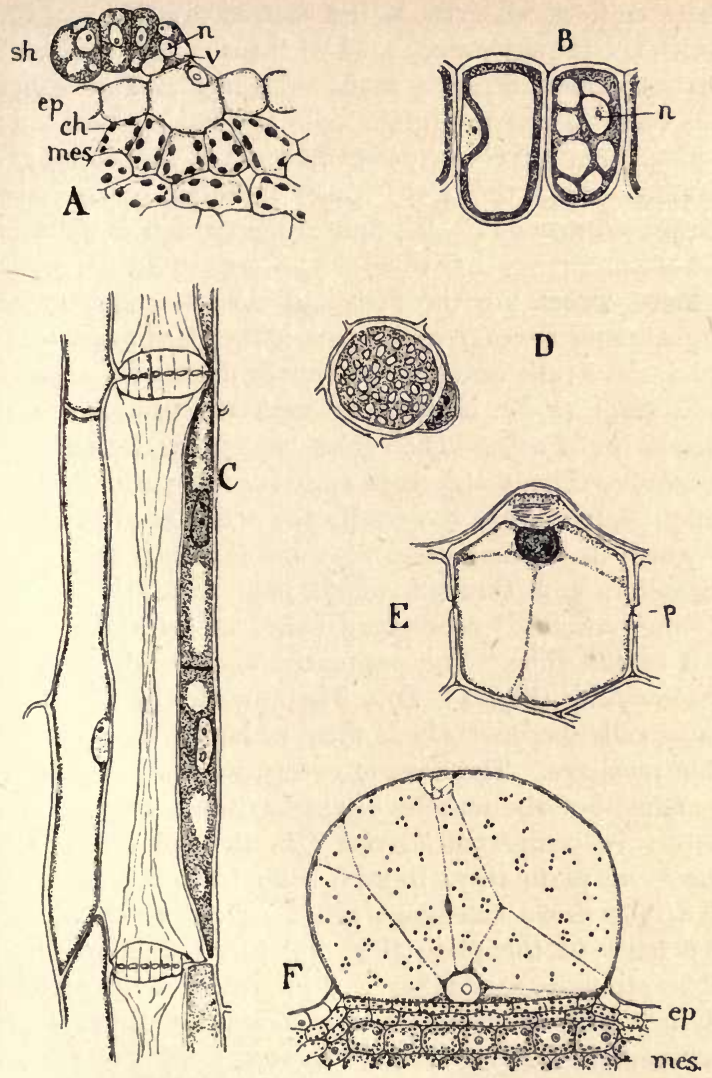

ordinary parenchyma cell. $\mathrm{D}$, sieve plate and companion cell as seen in cross-section. E, epidermal cell with large vacuole, but. large conspicuous nucleus in contact with local thickening of outer cell wall which is being formed; $p$, pit in lateral wall. F, large water bladder representing a single much swollen cell of the epidermis, with little cytoplasm but large nucleus; $e p$. , ordinary epidermal cells; mes., mesophyll. 
the secretory cell type, is the protein cell (Fig. 43, C), which is constantly associated with the cells (sieve tubes) that conduct organic food substances. The exact functions of these protein cells are not understood: perhaps they secrete enzymes which act on the substances passing along the sieve tubes. Protein cells have large conspicuous nuclei, and are very rich in protein contents.

Sieve Tubes are the main channels of transport of organic substances from one part of the plant to another. Each sieve tube consists of a row or chain of elongated cells (Fig. 43, C), comparable with the medullary cell chains of Fucus. The cross walls separating the successive cells of the chain early become perforated by holes-eaten out as it were by the action of an enzyme -which in extreme cases may be as much as $5 \mu$ in diameter, and through which pass relatively coarse "slime strands" connecting each cell with the next cell of the tube. The perforated cross wall is called a sieve plate (Fig. 43, D). The vacuoles of the sieve tube cells become filled with a highly nitrogenous thin mucilage. The layer of cytoplasm lining the walls remains, but the nucleus disappears, and the cells can hardly be considered "living" in the full sense. On the sieve plate there is eventually formed a mass of a carbohydrate substance called callose which blocks the holes in the plate (Fig. 43, C). Callose refracts light strongly and takes a deep colour with certain stains. The blocking of the holes by the callose apparently interrupts the transport of substances along the tube; later the callose is dissolved, and ultimately the sieve tube becomes empty and no longer functional, for instance in the parts of woody plants which are several years old. 
Each sieve tube cell has one or more companion cells alongside of it (Fig. 43, C). These are typical " protein cells" (see above). In most Seed Plants they are cut off by division from the mother cell of each sieve tube segment at an early stage of its development, before the sieve tube cell acquires its special character.

The sieve tubes are certainly the main conducting channels of organic nitrogenous substances (amino-acid compounds, amides and perhaps the simpler proteins) and of sugars, but we know very little indeed of the manner in which the transport takes place.

\section{Living Cells poor in Protoplasm. Water Tissue.-At} the other extreme from secretory and protein cells are water cells, which have a layer of cytoplasm lining the wall very thin in comparison with the size of the cell (Fig. 43, E, F), the vacuole being filled with a very watery sap, i.e. an extremely dilute solution. These cells are, however, only an extreme form of the typical thin-walled parenchymatous cell. The bodies of succulent plants consist mainly of such thin-walled water cells, but in the typical succulents (Cacti) the cells contain a large quantity of pentosans (substances having a similar relation to pentose sugars that the celluloses have to the hexose sugars) which attract water strongly. The active cells, for instance the photosynthetic cells near the surface of the succulent stem or leaf, can draw upon the store of water in the water tissue.

The epidermal cells act as water cells in an ordinary leaf and provide a small supply of water which is drawn upon by the mesophyll cells if they are losing water to the air more quickly than they can be supplied from the water-conducting system. In some cases isolated epidermal cells are many times the size of 
their neighbours, and act as special water bladders (Fig. 43, F). And the leaves of certain plants growing in places where they are specially liable on occasion to lose water quicker than it can be supplied have a many layered thin-walled epidermis, and this massive water tissue loses water to the mesophyll and partially collapses, bellows fashion, under such conditions. Thick leaves often possess a central water tissue.

Thick-Walled Cells-Pits.-Many of the living cells of a plant thicken their walls considerably after the cell has grown to its definitive size. This thickening of the wall is often general or uniform over the whole wall surface, and may be carried so far that the cell cavity is greatly reduced in volume (Fig. 44, C, D, E). Successive layers of cellulose are laid down by the cytoplasm, and the layering (stratification) of the wall can often be clearly seen under the microscope (Fig. 44, B, C). This appearance is supposed to depend upon the smaller or greater amount of water contained in successive layers of wall, making the cellulose more or less solid in consistency.

The continuity of the layers is, however, generally interrupted at certain spots, where the original wall, formed at cell division (middle lamella), is not added to. These thin places in the wall are called pits. Pits are always formed opposite to one another in adjacent cells of a thick-walled tissue (Fig. 43, E, p), so that the cell cavities are only separated by the thickness of the middle lamella (pit membrane). A pit is very commonly circular in section, so that it appears as a lighter circular area when the wall is seen in surface view under the microscope. The walls of a pit are commonly perpendicular to the middle lamella, so that the pit is of equal diameter from top to bottom (" simple pits "). 
But in some cells, particularly the vessels and tracheids of the water-conducting system (see below), the pit is narrower where it opens into the general cell cavity than at the bottom on the pit membrane. The walls thus overhang the pit, which is then said to be bordered
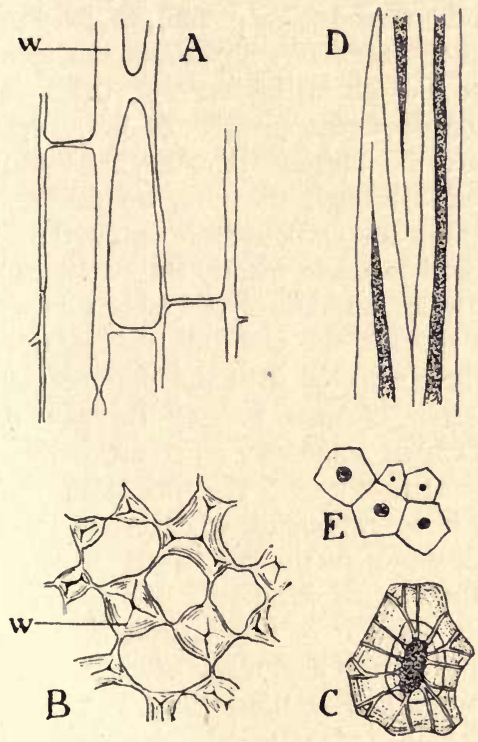

Fig. 44--Mechanical tissue elements of the Seed Plant. A, collenchymatous cells in longitudinal section, showing great thickening of parts of the walls forming longitudinal "pillars" of wall substance. $\mathrm{B}$, the same in cross-section; $w$, thickened walls. C, thick walled "stone cell" with branching pits. D, part of three fibres in longitudinal section. E, fibres in cross-section.

(Fig. 45, I). Some bordered pits are not included in the general thickness of the cell wall, but are built out, so to speak, into the cell cavity in the form of a flattish dome, with the pit opening at its summit. The two 
opposite pits of adjacent cells then form a lens-shaped structure on the cell wall. The extreme example of this type of bordered pit is seen in the tracheids (waterconducting cells) of conifers, for instance the commonpine.

The fine threads of cytoplasm, originally the achromatic spindle threads (see p. I06), connecting the cytoplasm of adjacent cells, are sometimes, though not always, confined to the pit membranes of thick-walled cells. The existence of pits greatly facilitates the passage of substances in solution from one thick-walled cell to another, and through the pits, no doubt, the sugar which supplies the carbohydrate material of which the thick cell wall is made mainly enters the cell. We do not know what determines the formation of a pit at any given spot on the cell wall, though in some cases it is clearly connected with the presence of a bundle of cytoplasmic threads passing through the middle lamella which in some way must arrest the deposition of cellulose at that spot. The formation of thick walls in general is an expression of excess of soluble carbohydrate substance in the cell, which is condensed and added to the wall as cellulose. In many cases, indeed, thick-walled tissue is of no particular use to the plant, but is simply formed as a result of carbohydrate excess, when photosynthesis is unchecked, but there is not a sufficient supply of salts to form proteins for the manufacture of new protoplasm. Thus most plants living in dry climates form great masses of thick-walled tissue. But thick-walled cells may be of great use to the plant, since they lend rigidity and toughness to its body (mechanical tissue).

Local Thickening of Cell Walls.-Sometimes the thickening of a cell wall is not uniform over the whole surface, but local: for instance in a box-shaped cell 
one wall only may be thickened. The external walls of the epidermal cells covering the shoot are generally considerably thicker than the lateral and inner walls, and sometimes the outer wall is very thick in the centre and lens-shaped (Fig. 43, E). In collenchyma, the supporting tissue of elongated living cells commonly met with just below the epidermis in herbaceous stems and in the midribs of leaves, the thickening is confined to the corners of the cells, so that longitudinally running pillars of cellulose are formed, connected with the neighbouring pillars by thin membranes (Fig. 44, A, B). Thus there is a certain amount of "give" under horizontal strain in this tissue. In many cells ribs or other local projections of various shapes are laid down on the inner surface of the wall. The spiral thickenings on the walls of the elaters of Pellia, and various similar thickenings in the cells of Seed Plants (Fig. 45, A-G), are cases in point.

Dead Cells.-In many cells of the plant body the protoplasm dies after the development of the cell is completed, the wall alone remaining. While these dead cells can no longer take an active part in the life of the plant, they often form an integral part of its structure, and many of them are of essential importance. The two chief functions they carry out are those of mechanically strengthening the plant body and of water conduction. The chief mechanical or supporting tissues are composed of thick-walled elongated cells with long tapering ends, which fit between one another, so that the tissue is very tough and not easily broken by strains. Such cells are called fibres (Fig. 44, D, E). The wall of the fibre is sometimes very thick, almost obliterating the cell cavity. It is usually penetrated by narrow pits, which serve to bring the sugars used 
for thickening into the cell. The protoplasm generally dies when the thickening is complete. The fibres are commonly found in bands or masses not very far from the surface of a herbaceous stem and in a similar position in the midrib and larger veins of the leaf, but they also form a large part of the wood of many woody plants, for instance the oak, to which they give great hardness and toughness. This depends partly on changes in the cell wall (see below). Fibrous tissue is used for making linen, canvas and paper, in which the fibres are woven together to make the textile fabric. The walls of the fibres in linen and the best paper are nearly pure cellulose.

Other thick-walled cells are box-shaped, spherical or oval, and when the walls are very hard they are called stone cells, which form the substance of the stone in stone fruits (plum, cherry, etc.), and isolated cells or nests of cells in the flesh of a gritty pear. Stone cells frequently have beautiful branched pits (Fig. 44, C), formed by the meeting and coalescence of several different pits as the inner surface of the cell is diminished by the progressive general thickening of the wall.

Masses of thick-walled cells near the surface of an organ may also have an important function in checking loss of water by evaporation.

The water-conducting cells of a plant are called tracheids and vessels (Fig. 45). These are dead cells with lignified walls (see below), which form continuous water-conducting channels throughout the plant parallel with the sieve tubes A tracheid is usually an elongated cell whose wall may be thickened in various ways.

(a) Spiral tracheids have one or more lignified bands of wall substance (Fig. 46) laid down on the inner surface of the wall and running round and round the 
cell through its entire length. The successive turns of the spiral are at first in close lateral contact (Fig. 45, A). The wall itself, apart from the thickening, usually remains thin and cellulose.

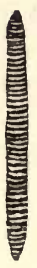

A

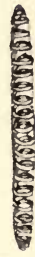

B

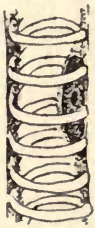

C
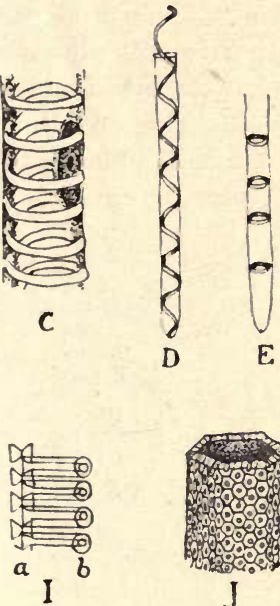

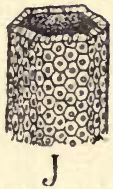

$\mathrm{H}$

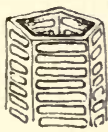

F1G. 45.-Water-conducting tissue elements. A, spiral tracheid, not yet elongated, surface view. B, elongation beginning, separating coils of spiral thickening. C, portion of B more highly magnified, showing nucleus and cytoplasm still alive. D, part of tracheid with coils of spiral further separated. E, ditto of annular tracheid. F, spiral tracheid with cavity nearly obliterated owing to pulling out of spiral thickening till it no longer supports the thin wall. G, ditto in annular tracheid. $H$, part of scalariform vessel. I, bordered pits: in (a) sectional view, (b) surface view (corresponding points joined by horizontal lines). J, part of pitted vessel. $K$, part of reticulate vessel.

(b) Annular tracheids (Fig. 45, E, G) are similar, but have the thickening in the form of separate rings of lignified substance, set one above the other, at first in close contact. 
Both these types are formed in tissue which is still elongating, and the stretching of the thin cell wall separates the coils of the spiral or the annuli, as the case may be (Fig. 45, B-G).

(c) Pitted tracheids have general thickening of the cell wall, but with numerous pits often set so close together (Fig. 45, J) that only ribs of thickening are formed between them. The pits are generally bordered, often with greatly overhanging walls (Fig. 45, I). When the pits are elongated and horizontal in direction, as seen in surface view, running across one face of a poly-

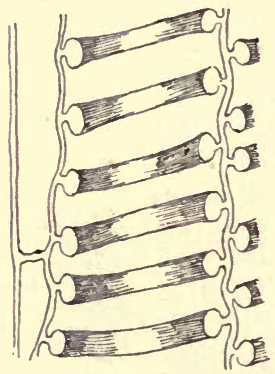

FIG. 46.-Part of a spiral tracheid very highly magnified and seen in longitudinal section, showing attachment of the spiral thickening band to the thin wall.

gonal tracheid, so that there are horizontal bars of thickening between successive pits, like the rungs of a ladder, the tracheid is called scalariform (Fig. 45, H). When the pits are angular and variable in outline, so that there is a network of thickening between them, we have the reticulate type $(\mathrm{K})$. The pits are, however, very often circular, oval or polygonal in outline and very closely set $(\mathrm{J})$.

A vessel has its walls lignified and thickened just like a tracheid, but it consists of a row or chain of 
cells with open communication between them, owing to the cross walls having been dissolved wholly or partially by the protoplasm before the cells were fully developed. A vessel is thus comparable with a sieve tube in that both are conducting structures formed of a chain of cells in continuity with one another. Vessels may be pitted, scalariform or reticulate, and have exactly the same function as chains of tracheids. They are, however, more efficient than chains of tracheids because, owing to the central perforation or complete disappearance of the cross walls, they offer less resistance to the passage of water. The wider water-conducting elements are always vessels. The widest tracheids are not more that Ioo $\mu$ in diameter, usually much less, while vessels commonly reach a width of $300 \mu$, and some measure as much as $700 \mu$ across.

\section{Alteration of the Substance of the Cell Wall.-The} walls of the living cells of the plant body commonly remain cellulose, but in many cell walls important changes affecting the functions of the cell takes place in the wall, usually while the cell is still alive, but sometimes after death.

One of the most important modifications is lignification. Lignification occurs in its extreme form in the walls of the tracheids, vessels, wood fibres, and often in the parenchyma of the wood. It also often affects other fibres to a greater or lesser degree. It depends on the deposition in the wall of lignocellulose, consisting of cellulose and two other constituents (an aromatic substance and a pentosan) often classed together as lignin. This gives characteristic colour reactions with various chemical reagents, such as phloroglucin and hydrochloric acid (magenta red), pyrogallol and hydrochloric acid (bluegreen), aniline chloride or aniline sulphate (golden 
yellow). Lignified walls also take up the aniline dyes strongly, so that the walls of the tracheids and vessels and often also of the fibres are deeply coloured in permanent microscopic preparations of plant tissues that have been stained with these dyes. Lignification hardens the wall considerably, but does not decrease its permeability to water.

In contrast to lignification, in which lignin is laid down in a cellulose matrix, is the formation of parts of certain cell walls, not by cellulose, but by one of two aggregate substances known as cutin and suberin. These are substances which differ chemically from one another, but are closely allied and have the same physical property of being impermeable to water. They are not true fats, but they are allied to fats and show some of the same reactions, for instance staining with Sudan 3. Cutin is the substance of the cuticle covering the epidermis of the shoot, and suberin forms one of the layers of the wall in cork cells. Hence both the outer walls of the epidermis of the herbaceous shoot and also cork tissue (which is formed in the bark of woody plants, see Chapter $\mathrm{XX}$ ) are practically impermeable to water, and thus prevent the drying up of the shoot by evaporation. The impermeability of cork to water accounts of course for the use of the bark of the cork oak (Quercus suber), which is very pure cork, to make bottle corks.

The contrast between cutin and cellulose can be demonstrated by treating a section through the epidermis with Schulze's solution (solution of iodine in zinc chloride) which stains the cellulose blue, owing to the formation of " amyloid," a substance that gives, like starch, a blue colour with iodine, but the cutin of the cuticle yellow. 
Another important substance of the cell wall is pectin, of which the middle lamella (the original thin wall formed at cell division) is often composed. Pectin is also formed in the cell walls (and cell contents) of succulent fruits. The pentosans (see p. 275), whicls, as we have seen, are characteristic of certain succulent plants, also occur in lignified cell walls, being the chief constituents of the "wood gums." The presence of these and other carbohydrates which readily take up water and become mucilaginous are the cause of the ready formation of slimy substances from many cell walls and cell contents on addition of water, for instance the swelling of the middle lamellæ of the medullary cells of Fucus, the swelling of the surface cells of linseed (p. 137), the breakdown into mucilage of the external cells of the root cap (p. 290), and many other cases. The process is the absorption of water by a carbohydrate gel, quite comparable with the swelling of gelatine considered in Chapter III (p. 53).

Intercellular Spaces.-Most of the living tissues of the higher plants are interpenetrated by a system of aircontaining spaces (intercellular spaces) initiated by the breaking apart of the cells along the plane of the middle lamella. The air in these spaces is often called the " internal atmosphere" of the plant. Its composition agrees more or less closely with ordinary atmospheric air, but except in tissues like the mesophyll of the leaf (Fig. Io), which are constantly setting free oxygen as the result of the photolysis of carbon dioxide, it contains a smaller proportion of oxygen and a larger proportion of carbon dioxide than atmospheric air, owing to the respiration of the living cells, which use up oxygen and set free carbon dioxide. The air in the intercellular spaces is normally saturated or nearly 
saturated with water vapour, because it is surrounded by living cells saturated with water. The exchange of gases between the living cells in the interior of the plant and the external air takes place by diffusion through this system of intercellular spaces.

\section{PRACTICAL WORK.}

(I) Ordinary living parenchyma. Several examples of this, with and without chloroplasts, have already been seen.

(2) Secretory (gland) cells. Examine demonstration specimens of sections through the enzyme secreting glands on the inner surface of the "pitcher" of the Pitcher Plant (Nepenthes) and also of sections through a nectary. Note in both cases that the cells are densely filled with cytoplasm and have conspicuous nuclei.

(3) Thick-walled cells, local thickening of cell walls, secretory cells and protein cells in transverse section of leaf of pine (a permanently stained and mounted section of a large-leaved species, such as $P$. pinaster, is best).

Examine first the thick-walled cells in the corner of the leaf. Those just below the surface layer have abundant cytoplasm and conspicuous nucleus-they are still active. Note the stratification of the walls, the middle lamella, and the pits. The cavities of the epidermal cells are almost obliterated. Note the thick layer of cuticle on their outer surfaces.

The mesophyll (photosynthetic) cells below the thick-walled tissue have numerous choloroplasts and plate-like projections of the walls (local thickenings) into the cavities.

Note the resin canals at intervals (one in each corner of the leaf). These are intercellular channels surrounded by secretory cells, and (outside these) thick-walled cells. The resin is expelled by the secretory cells and accumulates in the canal.

Examine the protein cells on the outer flanks of the central pair of bundles. These are rich in cytoplasm and have conspicuous nuclei. They adjoin the sieve tubes of the bundles.

Note also the lens-shaped bordered pits on the walls of some of the cells around the bundles.

(4) Stone cells. Tease out on a slide in a drop of dilute glycerine a little of the flesh of a pear, and note the thick-walled pitted stone cells, singly and in groups, among the thin-walled parenchyma. When the stone cells are numerous the pear is gritty between the teeth. 
(5) Sieve tubes and companion cells. Examine the demonstration preparations of sieve tubes and companion cells in transverse and longitudinal section of the stem of Cucurbita. Note the thick perforated sieve plates (cross walls of sieve tubes), the callose, the cytoplasm contracted from the side walls of the tube but not from the plates, and the narrow companion cells (protein cells) with granular contents and conspicuous nuclei. Measure with the micrometer eyepiece the diameters of a sieve tube, of a companion cell and of the pores of the sieve plate.

(6) Vessels, fibres and wood-parenchyma. Examine a piece of Cucurbita stem and identify the longitudinally running strands (bundles) with large openings (vessels) visible to the naked eye. Tease a little of a previously macerated bundle from the stem of Cucurbita in a drop of dilute glycerine, and observe the narrow spiral and annular vessels and pieces of the broad reticulate vessels. Measure their diameters. Thin-walled parenchyma cells are associated with the vessels. Treat similarly a little macerated willow wood and note that it consists mainly of fibres-long narrow cells with thick walls and tapering endsand of a few vessels bearing close-set bordered pits where they abut on one another. Measure the diameter of a vessel. Note also the thick-walled oblong cells with simple pits running through the wood in plates (medullary rays).

(7) Water tissue. Note the massive thin-walled tissue forming the bulk of the succulent leaf of Kleinia (or other "leaf succulent "). Contrast this with the layer of tissue containing chloroplasts near the surface. The green cells draw on the water tissue when they lose water more rapidly than it can be supplied through the bundles from the roots.

Examine transverse sections of the fresh Begonia leaf and note the many layers of thin-walled colourless cells on the upper side. This is derived from the upper epidermis by divisions parallel with the surface. These cells are living, and the very thin cytoplasmic lining of the cell walls can be seen here and there. The water tissue on the lower side contains chloroplasts, and is not derived from the epidermis but from the mesophyll tissue. It is, however, very distinct from the main photosynthetic layer in the centre, which is densely packed with chloroplasts. 


\section{CHAPTER XVII}

\section{THE ROOT}

HAviNG now obtained some knowledge of the appearance and structure of the elements of which the tissues of the bodies of the higher plants are composed, we pass on to consider the nature, structure and functions of the main vegetative organs of the plant-the root and the shoot (stem and leaves)-aggregations of these tissues.

Roots are colourless axes of the plant which typically descend into and grow in the soil, fixing the plant and absorbing water and dissolved salts from the soil. It is pretty certain that they are to be regarded as specialised underground branches of the thallus which formed the body of remote ancestors of the higher plants. According to their origin in the life of the individual seed plant we distinguish the primary root system, i.e. the taproot or main descending axis of the plant (already laid down in the embryo) and its branches, from the adventitious roots which arise from some part of the shoot, e.g. from a rhizome or from the lower part of an erect stem (see Chapter XV, p. 26I). These two kinds of roots do not, however, differ structurally in any essential respect.

Tropisms of Roots.-Some of the most important physiological characters of roots are their distinctive tropisms (see Chapter V, p. 87). The taproot is in the great majority of cases positively geotropic, i.e. it grows towards the centre of the earth, and if it is placed 
in a horizontal position the region just behind the tip bends so as to bring the apex pointing vertically downwards. This bending is carried out by the cells on the upper side of the elongating region growing faster than those on the lower side, but how the root perceives the stimulus of gravity is not by any means fully understood. The branches of the taproot do not grow vertically downwards but obliquely, making some angle less than a right angle with the continuation of the main root.

Many roots are also negatively phototropic, i.e. they tend to grow away from a source of bright light. The strongest tropism of roots is, however, positive hydrotropism, or the tendency to grow towards a region of greater moisture. In the case of a taproot growing in ordinary moist soil these three tropisms will clearly all act in the same direction, they will all take the root directly downwards, for not only is this the direction of the pull of gravity, it is the direction away from the light, and it is also the direction of greater moisture, for the surface layers of soil will be drier than the deeper ones as a result of evaporation to the air. But if the soil is rather dry and the source of moisture is to one side, then the roots will bend in that direction, the hydrotropic tendency overcoming the others.

Growing roots require a supply of free oxygen for respiration, and the roots of most ordinary land plants flourish best and develop root hairs (their absorbing organs) most abundantly in well aerated soil, in which the air is saturated or nearly saturated with water vapour.

Structure.-The apical (primary) meristem of the root is covered by a root cap (Fig. 47, A, r.c.), thickest at the apex and gradually thinning at the sides till it comes to an end a short distance behind the tip. The 
root cap is composed of living cells derived from the primary meristem $(p . m$.) from which they are continually renewed by active cell division, while those on the surface of the cap break down into mucilage and are rubbed off as the root tip is pushed through the soil. Behind the root tip (Fig. 47, $a$, A) there is a bare stretch of varying length, often about 5 to Io $\mathrm{mm}$. long, where the root is actively growing in length: this is the elongating region (E). Behind this again is the roothair region (R), thickly covered under favourable conditions of soil moisture and aeration, with root hairs, each of which is a tubular outgrowth from a single cell of the surface layer (piliferous layer) (Fig. 48). The root hairs come into intimate contact with moist soil particles and absorb water owing to the greater osmotic strength of the cell sap than of the dilute solution outside, and also salts in solution. It should be understood that the entrance of the salts into the root hair is independent of the entrance of water, and depends primarily on the difference of concentration of the various solutes inside and outside the root-hair cell. The ectoplasm of the root hairs, which is impermeable to the "osmotic substances" in the cell, must of course be permeable to these salts. New root hairs are formed in front of the root hair region by the growing out of the cells of the piliferous layer into papillæ which elongate to form the hairs (Fig. 49, A). The hairs at the back usually die, but in some cases they are persistent and clothe the root for a long distance even to the point of origin of a root several inches long. But ordinarily the life of a root hair is short, and behind this region the root is again bare.

Growth of the Root Tip.-As new cells are formed by division of the meristem the ones already formed pass 
in to the elongating region, increase actively in length by the formation and enlargement of the vacuole, and
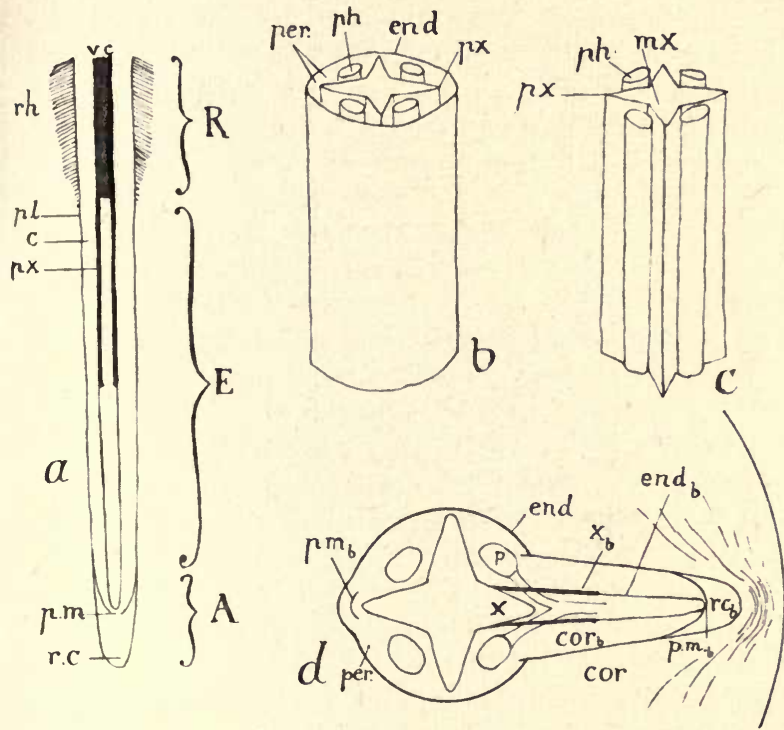

FIG. 47.-Diagrams illustrating the structure of the root. $\mathbf{a}$, in longitudinal section. A, apical region; $\mathrm{E}$, elongating region; $\mathbf{R}$, root-hair region; $\boldsymbol{r} . c$. root cap; p.m., primary meristem; v.c., vascular cylinder; $p x$., protoxylem; $c$, cortex; p.l., piliferous layer; $r . h .$, root hairs. b, part of vascular cylinder (the conjunctive tissue of the cylinder is supposed to be transparent, the endodermis opaque). c, the same with endodermis removed; end., endodermis; per., pericycle; $p h .$, phloem; $p x$., protoxylem; $m x$., metaxylem. d, Diagram of cross-section of cylinder through origin of branch root; cor $_{b}$, end $_{b}, x_{b}, p . m_{b}, r . c_{b}$, cortex, endodermis, xylem, primary meristem and root cap of branch root. On the left the primary meristem of the opposite branch is just formed in the pericycle. The root in $\mathbf{b}-\mathrm{d}$ is tetrarch, i.e. with 4 xylem and 4 phloem strands.

gradually become differentiated into the various tissues. The force of elongation of the cells in this region exerts 
a push up and down the root. The region behind the elongating region is held fast in the soil by the root hairs, but the root apex, covered by the root cap, is

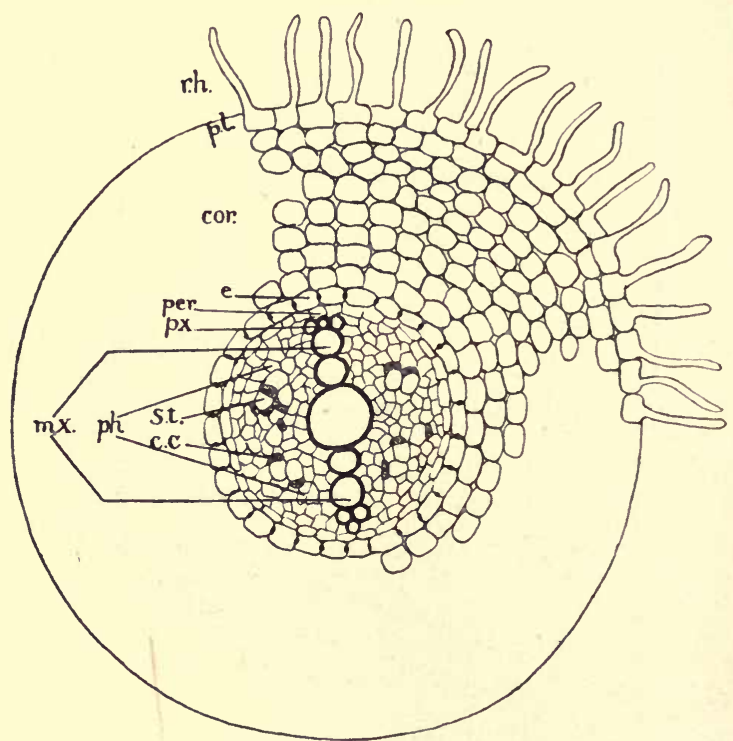

FIG. 48.-Cross-section of a small root taken in the root-hair region : r.h., root hairs; p.l., piliferous layer; cor., cortex; $e$, endodermis; per., pericycle; $p x$., protoxylem; $m x$., metaxylem; $p h_{\text {, }}$. phloem (s.t., sieve tube; c.c., companion cell). This root is diarch, i.e. with 2 xylem strands, joined to form a plate, and 2 phloem strands.

pushed between the particles of soil, its passage being lubricated by the mucilaginous surface of the cap.

Internal Structure.-A cross-section of the root taken in the middle of the root-hair region gives the best idea of the primary plan of construction of the . root. On the surface is the piliferous layer (Fig. 48, p.l.), 
each cell of which is capable under favourable conditions of growing out to form a root hair $(r . h$.$) . Within$ is the cortex (cor.), composed of thin-walled parenchyma interpenetrated by intercellular spaces. In the centre is the vascular cylinder, containing the conducting (vascular) system.

The vascular cylinder is separated from the cortex by a single layer of cells, the endodermis I (Fig. 48,e). The lateral and transverse cell walls, i.e. those walls which are common to adjacent cells of this layer, or the central strips of these walls (Fig. 49, B), are composed of cutin (see p. 284) : the outer and inner walls of the endodermis, i.e. those abutting on the cortex and the tissues of the cylinder respectively, are cellulose. Thus the endodermis is stiffened and united into a more or less rigid layer whose cells cannot be separated (Fig. 49, C), and there is a free passage for water and solutes from cortex to cylinder only through the inner and outer walls and through the bodies of the endodermal cells.

Immediately below the endodermis is a layer of parenchymatous cells, the pericycle (Fig. 48, per.), ${ }^{2}$ generally only one cell thick. Below and immediately abutting on the pericycle come the vascular (conducting) clements proper, arranged in alternating strands of tracheids and vessels-xylem 3 and sieve tubes (s.t.) with companion cells (c.c.)-phloem. 4 The xylem and phloem strands are separated from one another by

I Greek $\tilde{\varepsilon} \nu \delta o v$, within, $\delta \varepsilon ́ \rho \mu \alpha$, skin, i.e. the inner skin, the skin of the vascular cylinder. This is not to be verbally confused, as is often done by beginners in biology, with the "endoderm," the lining of the primitive gut of animals. The terms are both derived from the same Greek words, but have a very different technical meaning. It is therefore important to keep the distinctive ending.

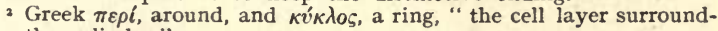
ing the cylinder."

3 Greek $\xi u ́ \lambda o v$, wood - the wood of a tree is secondary xylem, see p. $33 \mathrm{r}$.

4 Greek, $\phi$ גoı́s, bark-the inner bark of a tree is secondary phloem (p. 340$)$. 


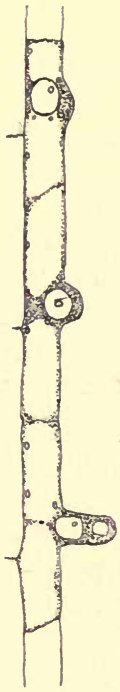

A

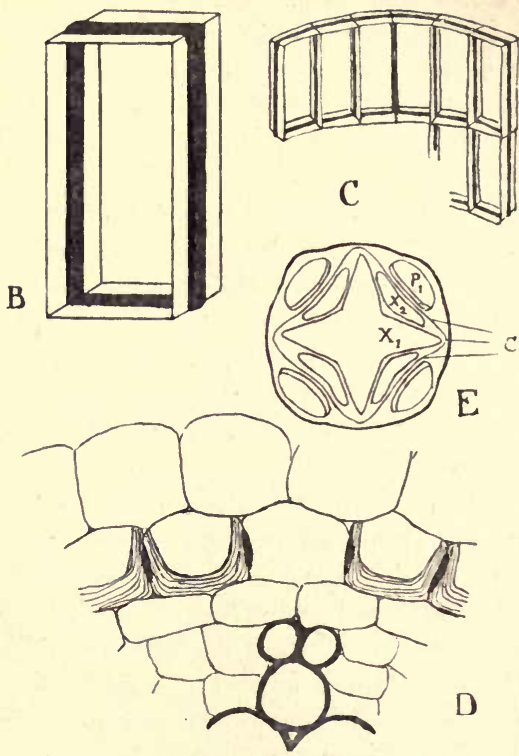

FIG. 49.-A, three cells of the piliferous layer at the beginning of the root-hair region in longitudinal section, showing the early stages of the development of root hairs. Note the accumulation of cytoplasm and the presence of the large nucleus (which passes into the hair) at the point of origin of the root hair. B, diagram of single endodermal cell, showing the cutinised band (black) round the radial and horizontal walls. The tangential walls (front and back) are supposed to be transparent. C, diagram of portion of endodermis, supposed to be seen from within the cylinder, showing the way the cells are fitted together and "cemented" by the cutinised bands. D, small portion of edge of cylinder in an old and large root, showing "horse-shoe" thickening of endodermis, and "passage cell" opposite protoxylem. E, diagram of cross-section of vascular cylinder in which secondary thickening has begun, the secondary tissue at present formed only opposite the primary phloems. $X_{\mathrm{I}}$, primary xylem; $X_{2}$, secondary xylem; $P_{1}$, primary phlom; $C$, cambium. 
parenchymatous rays which are continuous with the pericycle. In slender cylinders, where the number of alternating strands of xylem and phloem is small the centre of the cylinder is filled up with large xylem vessels in contact with the strands of xylem which abut on the pericycle (Fig. 48). In massive cylinders where the number of xylem and phloem strands is large the centre is occupied by parenchyma (pith).

The outermost tracheids, abutting on the pericycle, are narrow, and spiral or annular. They are the first xylem elements to be formed, and are hence called protoxylem (Fig. $48, p x$.). Within these are larger pitted vessels (metaxylem) (Fig. 48, $m x$.), which, in a narrow cylinder, fill up the centre (Fig. 48). The spiral or annular thickenings of the outermost tracheids are laid down and lignified in the elongating region of the root, the protoplasm dying after the thickenings are complete. The thin cellulose wall of the tracheid is passively stretched by the active elongation of the surrounding parenchymatous tissue, and the successive turns of the spiral thickening (or the annuli) are pulled apart (Fig. 45, B-G). As long as they are not too widely separated, the internal thickenings of the tracheid prevent the obliteration of the cavity by the pressure of the surrounding turgid tissue, and so this first formed water-conducting channel is kept open.

Thus the spiral and annular tracheids are very beautifully adapted to perform their function under the conditions in which they are formed, for they both admit of longitudinal extension to keep pace with the growth in length of the tissue, and are protected against obliteration by the surrounding turgid tissue. In organs which elongate a great deal, the spiral tracheids first formed are ultimately stretched so much 
that the thickening no longer supports the wall and the tracheids are crushed (Fig. 45, F).

A transverse section across the elongating region shows the protoxylem elements already lignified, but the cells which will develop into metaxylem vessels still thin walled and living. In the root-hair region the walls of these gradually thicken and lignify, and various stages in their development can be traced.

Absorptive Function of the Root.-Owing to the existence of substances such as sugar, which attract water, in the cell sap of the root hair, water is drawn in from the soil outside. As the solution within the vacuole becomes more dilute, the absorptive power of the root-hair cell decreases, and when the dilution has passed a certain point the absorptive power of the adjoining cortical cells, whose cell sap will then be more concentrated than that of the root-hair cell, will cause water to be drawn into them from the root-hair cell. In the same way water will be drawn across the cortex from cell to cell through the endodermis and pericycle to the xylem tracheids and vessels. The mechanism of the passage of water from the living cells of the cylinder into the xylem tracheids and vessels is not fully understood, but it seems probable that osmotically active substances exist in considerable quantities in these also. When they lose their protoplasm after differentiation a considerable amount of organic substance must be left in their cavities.

It must be clearly understood that the passage of solutes, for instance the mineral salts-nitrates, sulphates and phosphates-absorbed from the soil and ultimately passing into the xylem, do not necessarily travel across the cortex at the same rate as the water. Their passage depends on the state of equilibrium between the different 
cells in regard to each salt, and on the relative permeability of the cytoplasmic membranes to the different salts.

Structure of the Older Parts of the Root.-In exceptional cases, as has already been said, root hairs may persist indefinitely during the life of the root, but normally their life is quite short, and they die and peel off, with the piliferous layer, behind the limited root-hair region. The surface of the root is then formed by the outer cells of the cortex, and the outer walls of these cells, now in contact with the soil, are cutinised and no longer absorb water from the soil. Meanwhile the metaxylem vessels of the vascular cylinder are now completely lignified, and the conducting capacity of the primary structure has reached its fullest extent. Secondary changes may now occur :-

(I) Branching.- Branch roots arise in this region, by the division of the cells of the pericycle, usually opposite a protoxylem strand. A new apical meristem is established, covered by a root cap, and the tip of the new root grows out through the endodermis and cortex of the mother root (Fig. 47, d), partly by digesting the cells, partly by mechanical pressure, and emerges into the soil. When a root is branching freely a fairly close-set row of branches may grow out from opposite each protoxylem of the mother root, so that if the mother root is tetrarch, four such rows of branches will appear. The vascular tissues of the new root establish connexions with the corresponding tissues of the mother root; thus the xylem strands connect with the xylem opposite which the new root has arisen by the differentiation of tracheids in the intervening tissue, the phloems by the formation of sieve tubes connecting with the sieve tubes of the two phloem 
strands on each side of the point of origin. In this way the conducting systems of the mother root and of the branch roots become part of one system. The cortex of the branch, however, never has any connexion with the cortex of the mother root.

(2) Secondary Thickening.-In perennial plants which have a persistent primary root system, and also in many annuals, the primary tissues are added to by the activity of a secondary meristem. In other words, certain living cells of the primary tissue begin to divide, and the products of their division become differentiated to form new permanent tissues, which are called secondary tissues, especially new (secondary) xylem and phloem. This process takes place in the roots and stems of all the woody plants, and to a certain extent in most herbaceous plants belonging to the Dicotyledons (the largest group of seed plants) and to the Conifers (pines, firs, etc.). The secondary vascular meristem is called the cambium, and its activity results in the great and continual increase in thickness which occurs in the stems and roots of trees. We shall have to consider it again in connexion with the woody stem, but we may here note the beginning of the process in persistent roots.

The formation of the cambium starts by the tangential division of the conjunctive cells just inside and on the flanks of the primary phloem strands. The cells cut off on the inside, i.e. towards the centre of the root, become new (secondary) xylem, the strands so formed alternating with the primary xylem strands (Fig. 49, E). A few sieve tubes are cut off on the outside of the cambium and added to the primary phloem. The cambium now extends round the outside of the primary xylem strands so as to form a complete layer round 
the cylinder. This layer forms a wavy line as seen in cross section, sweeping out round the primary xylem and in round each primary phloem (Fig. 49, E, c). Opposite the primary xylems it sometimes forms nothing but parenchymatous tissue (secondary rays), but in other species it forms xylem to the inside and phloem. to the outside, just as it does opposite the primary phloems. Owing to its greater activity at first opposite the primary phloems and to the formation of hard masses of secondary xylem in these regions the cambium soon straightens out and becomes circular in crosssection. In the root of a tree it continues its activity year by year, and eventually forms a bulky cylinder of secondary wood, and a much thinner cylinder of secondary phloem.

(3) Cork Formation.-Another secondary meristem besides the cambium also arises in woody roots, usually in the pericycle. This is called the cork cambium or phellogen, because it cuts off cells to the outside, certain layers of whose walls are formed of suberin. These are called cork cells, and owing to the impermeability of the corky walls to water and solutes, the cortex and endodermis are cut off from any functional connexion with the vascular cylinder and soon scale off. This formation of " bark" on woody roots is quite parallel to that which takes place on tree-trunks. On the inside the phellogen often forms parenchymatous tissue, which is sometimes called the "secondary cortex."

Modified Roots.-In some species the roots, or some of them, depart more or less from the typical root structure and behaviour. In some plants (climbers and epiphytes) they may turn green and carry on photosynthesis for the plant. Later they may grow down to and enter the soil, then assuming typical 
root characters. In parasitic seed plants organs called haustoria of very variable structure bore into the tissues of the host and absorb food from it. Sometimes these haustoria (Thesium, Rhinanthus) are just modified branches of ordinary soil roots, which fasten on and bore into the roots of other plants. In other cases (Mistletoe) the parasite never has any connexion with the soil at all, but the seed germinates on a tree, and its rootlike haustoria bore into the tissues of the tree, obtaining water and mineral salts from the xylem. In other cases, again (dodder), the haustoria are strands of tissue (in which sieve tubes may be developed) that tap the sieve tubes of the host. And finally, in the most extreme cases (Rafflesia, a tropical parasite), the whole body of the parasite consists simply of a branching mass of strands of tissue, like the hyphæ of a fungus, which live entirely in the tissues of the host, occasionally coming to the surface and producing immense flowers.

In some plants (carrot, turnip, parsnip) the main taproot is greatly swollen, and acts as a food storage organ through the winter in the same way as a corm or tuber. The great mass of fleshy tissue is produced by the cambium, and may be reckoned as parenchymatous secondary xylem and phloem, in which a few strands of conducting elements are formed here and there.

\section{PRACTICAL WORK.}

(I) Draw a single mustard seedling that has been germinated in moist air (being careful to keep the root moist), and note the long densely crowded root hairs, not yet full grown towards the tip of the root, and the bare elongating region between the root hairs and the apex. Distinguish root from hypocotyl. Compare with seedlings grown in soil, and in the latter note the clinging of the root hairs to soil particles.

(2) Transfer a seedling to a slide, just covering the end of the root with water under a coverslip, and examine under the micro- 
scope. Note especially the region of short (young) root hairs, each of which arises from a surface cell. Note also the region of primary meristem covered by the root cap, the bare elongating region and the vascular cylinder seen through the semi-transparent cortex.

(3) Examine the fresh taproot, 2 or 3 inches long, of a Bean seedling. Observe the root cap covering the growing point and the four or more longitudinal rows of lateral roots. $\mathrm{Cut}$ the root longitudinally through the centre in the region of origin of the branch roots, and make out with a lens that these arise from the surface of the central cylinder.

In the older part of the root the cortex can generally be separated from the cylinder by twisting the root so as to break the cortex and then pulling it off.

(4) Make a low power diagram of a transverse section of a buttercup root taken through the root hair region, and showing piliferous layer, cortex and cylinder, and in the cylinder the crossshaped xylem with four arms, the ends of which are formed by the narrow tracheids of the protoxylem. Between the arms of the xylem are the four strands of phloem. Put in the boundaries of the tissue regions, but not individual cells.

With the high power examine the different tissues in detail, and draw a portion of the cylinder, including a segment of endodermis and pericycle, protoxylem, large vessels of the metaxylem (in the centre these still have thin walls-cytoplasm and nucleus can sometimes be seen in them), also sieve tubes and companion cells of the phloem, and conjunctive tissue. Starch grains can be seen in the cortex.

(5) Examine the cross-section of a young Bean root, noting that the outline of the cylinder is not so easy to define as in the Buttercup, the endodermis being less sharply distinguished from neighbouring cells, but on careful examination with the high power the cutin strips of the radial walls can be made out. The xylem strands are not joined in the centre, which is occupied by pith. The thicker walled cells forming the bulk of the phloem are fibres: a few sieve tubes can be seen outside these. In older sections cambium (formed early here) and secondary tissues can be made out.

(6) Examine longitudinal sections through the centre of the tips of Maize and Bean roots. Note in each root cap, primary meristem, and the growth in size and elongation of the cells as the apex is left. In the Maize especially note the great increase in width of the rows of cells which will form the large vessels, and the stages of development of these. 


\section{THE FOLIAGE LEAF}

THE leaves and stems of the higher plants are differentiated organs of the shoot. In most of the lower chlorophyll-containing plants (Fucus, Pellia) the shoot does not show this differentiation, but it is established in all the vascular plants, except a few peculiar forms which have lost it. The foliage leaf is essentially the organ of photosynthesis, its principal tissue, the mesophyll, containing the great majority of the chloroplasts of the plant, though the parenchymatous tissue of the stem generally contain a certain number.

Essential Structure.-The typical foliage leaf (Fig. 50) is essentially a thin plate of mesophyll, thin enough to allow light of sufficient intensity to reach all the cells, with air spaces between the cells, communicating with the outer air, and thus permitting free diffusion of gases between the cells and the air. The mesophyll is interpenetrated by vascular bundles (veins) which carry water and salts coming from the root (through the vessels and tracheids of the xylem) to the mesophyll, and formed organic substances away from it (through the sieve tubes of the phloem) to other regions of the plant, principally to growing regions and storage organs. The mesophyll is protected by a layer of cells (the epidermis), typically not containing chlorophyll and acting as a water tissue (see p. 275), and the continuous outer layer of the 
external walls of these cells, in contact with the outer air, consists of a waterproof covering, the cuticle, composed of cutin (see p. 284). If the cuticle were perfectly continuous over the whole surface of the leaf it would prevent the interchange of gases between the mesophyll and the outer air, for instance the supply of carbon dioxide which the chloroplasts use as part of the raw

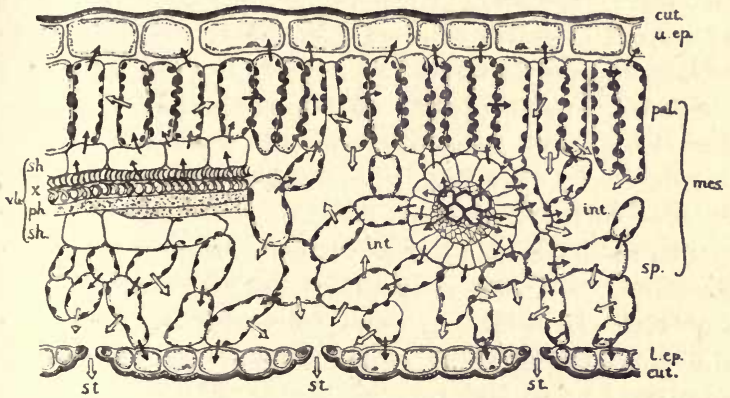

FIG. 50.-Part of transverse section of dorsiventral foliage leaf: cut., cuticle; u.ep., upper epidermis; mes., mesophyll ; pal., palisade tissue ; $s p$. , spongy tissue ; int., intercellular space, v.b., vascular bundle (in longitudinal section, passing out of the plane of section to the right-further to the right a bundle is seen cut in transverse section) ; sh., bundle sheath ; $x$, xylem; ph., phloem ; l.ep., lower epidermis; st., stoma. The black arrows indicate the passage of liquid water from the xylem tracheids through the sheath into the mesophyll cells and epidermal cells. The white arrows the evaporation of water from the mesophyll cells into the intercellular spaces and diffusion out through the stomata.

material of photosynthesis. The epidermis, with its cuticle, is in fact pierced by minute holes (stomata), each enclosed by two specialised epidermal cells (guard cells), and these pores lead into the system of intercellular space of the mesophyll (Fig. 50).

Transpiration.-The pores of the stomata not only allow of the diffusion of carbon dioxide from the air 
into the leaf as it is used up by the chloroplasts of the mesophyll cells, but also, of course, allow the water vapour evaporated from the saturated mesophyll cells into the intercellular spaces to diffuse out to the (usually) drier air outside the leaf. This process is called transpiration.

Large quantities of water are lost by the plant in this way. If a plant growing in a flower-pot is weighed, and after a certain interval weighed again, the loss in weight represents almost entirely transpired water, for the gain or loss in weight from photosynthesis or respiration is negligible by comparison with the loss by transpiration. The pot itself and the soil must be carefully covered with a metal or rubber covering waxed to the stem round the hole through which the stem passes, to prevent loss of water by direct evaporation from pot and soil. If desired, transpiration from the surface of the stem through the stomata it bears can also be prevented by waxing its surface. In this way it can be shown that it is through the leaves that the loss of water in transpiration mainly occurs. A sunflower plant whose total leaf area was 5,6I6 square inches lost $I \frac{1}{2}$ pints of water in I2 hours; and it has been calculated that the trees in an acre of beech forest transpire about I, 400 tons of water during the summer.

This constant loss of water from the leaf is probably useful to the plant because it helps to set up a current (transpiration current) through the xylem vessels and thus to bring up dissolved salts from the root more rapidly. That active transpiration is not necessary to the plant, or at least not to some plants, is shown by the fact that growth continues in tobacco plants, for instance, which are kept in air saturated with water vapour, so that transpiration takes place very slowly 
indeed. Evaporation from the mesophyll cells does however, take place even in saturated air, because the living cells, owing to the process of respiration, which is always going on and which liberates heat, are kept at a somewhat higher temperature than the surrounding air.

Structure and Mechanism of Stomata.-We have seen that if the leaf were covered by a perfectly continuous layer of cuticle it would lose little or no water, but it could not obtain carbon dioxide from the air. The stomata allow of the carbon dioxide getting in, but they also allow of water vapour getting out, and though this may be useful to the plant it introduces the danger of water loss so rapid that it cannot be covered by a corresponding supply from the root. This danger is partly met by the automatic closing of the stomatal pore when there is a deficiency of water in the tissues of the leaf. The guard cells of the stoma which surround the pore, unlike the other epidermal cells, contain chloroplasts (Fig. 5I, C, E), and are thus able to make sugar and maintain a high osmotic pressure and power of ahsorbing water. The guard cells are firmly joined to one another at the two ends, but in the centre there is a split (the stomatal pore) between them, while the side walls away from the pore abut on, and can be pushed into the cavities of, the adjacent epidermal cells. The cavity of the guard cells is often confined to the middle part of the cell as seen in vertical section: above (next the outer air) and below (next the intercellular space into which the pore leads) the wall is thick and cutinised.

When the guard cells become turgid the cell cavities increase in size, and since the ends and the top and bottom are firmly held, the side walls increase in length, 
the outer ones bulging into the adjacent epidermal cells, the pressure of whose cell sap is lower, and the
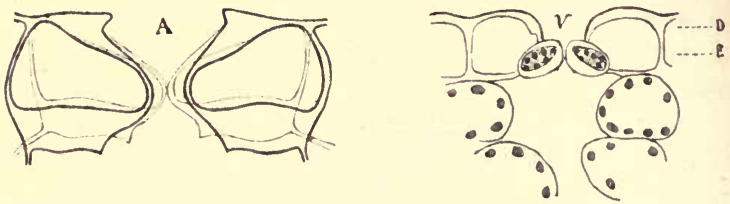

B

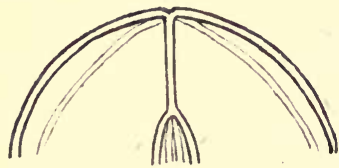

C

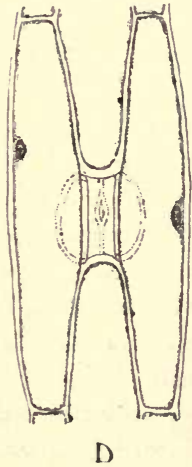

FIG, 51.-A, diagram of stoma seen in vertical section. The thin lines represent the position of the walls of the guard cells when the stoma is closed, the thick lines when it is open, the outer lateral walls bulging into the adjacent epidermal cells. $B$, the same in surface view (half the stoma only shown). C, stoma of Iris leaf in vertical section, showing the guard cells sunk below the surface of the epidermis at the bottom of the vestibule $(v)$. $D$, the same in surface view: epidermal cells focussed, the outlines of the guard cells seen faintly below. $E$, the guard cells focussed. F, ending of a fine vein in the mesophyll : one line of tracheids surrounded by sheath cells with few chloroplasts. 
inner ones curving away from each other and thus opening the pore (Fig. 5I, A, B, heavy lines). This is the normal condition in bright light and when there is plenty of water in the leaf. If the air outside becomes very dry water vapour diffuses out through the pores very rapidly, and thus the vapour pressure in the intercellular spaces of the mesophyll is reduced. This in turn increases the rate of evaporation from the mesophyll cells into the intercellular spaces and depletes the cells of water. Water is drawn into the mesophyll cells from the epidermal cells and eventually into these from the guard cells of the stomata. The guard cells in consequence lose their turgor and become flaccid, closing the pore by the straightening of the extensible portion: of the walls (Fig. 5I, A, B, thin lines). ${ }^{\mathrm{I}}$ At night also, owing to the cessation of sugar formation by the chloroplasts of the guard cells, the osmotic pressure falls and the pore closes.

This automatic mechanism closing the stomata when the supply of water is depleted does not always completely stop the loss of water from the plant, as may be seen towards the close of a hot dry day in summer, when the leaves of some plants become limp and droop owing to the continued loss of water to the air, a loss which they are unable to make good from the root quickly enough to maintain the turgor of the cells. This may be due partly to the incomplete closure of the pores, and partly to the fact that the cuticle is not completely impermeable to diffusion of water vapour. A drooping plant will gradually recover if a bell jar be placed over it. The jar prevents the removal by currents of air of the vapour

- This mechanism can be imitated by cutting off a piece of the inner tubing of a bicycle tyre ro inches long ( 5 inches on each side of the valve), tying up the two ends tightly and fixing them to a straight piece of wood so that the concave side of the tube lies along the wood. If air is pumped into the valve the tube curves away from the wood as it becomes inflated, and straightens as it is deflated again. 
escaping from the leaf, and the accumulation of this round the leaves checks further diffusion from their intercellular spaces, and thus enables the turgor of the leaf cells to be recovered by the retention in the leaf of the water coming from the root.

Most land plants possess structures which tend to produce the same sort of effect as the bell jar placed over the plant, i.e. the prevention of the rapid removal by air currents of the water vapour escaping through the stomata. The commonest of these is the sinking of the stoma in a pit (vestibule), the bottom of which, occupied by the guard cells and pore, is sunk below the general level of the cuticle (Fig. 5I, C). Sometimes there is a deep groove or cavity in the leaf containing several stomata. In other cases the leaf rolls or folds up in dry weather, thus protecting the surface bearing the stomata. In other cases, again, there is a thick covering of hairs either over the whole surface bearing stomata or confined to the cavities in which the stomata are sunk. All these structures serve the same purpose-to keep the air just outside the stomata relatively still and thus prevent the rapid removal of water vapour by currents of air, which is a very much quicker process than the slow diffusion, which continues in any case. The drying effect of wind is well known, and the shoots of plants exposed to strong, and especially of course to dry, winds, very quickly wilt and even die (through loss of water greater than they can recover), unless they are very well protected in one of the ways described. It is among plants living in very dry climates, or frequently exposed to strong winds during the growing season, or growing in dry soil, that the structures described are most developed, for it is only such plants (xerophilous $x$

× Greek $\xi \eta \rho o ́ s$, dry; $\phi i \lambda o s$, friend. 
or xeromorphic plants), thoroughly well protected against water loss, that can survive in these habitats.

Structure of the Mesophyll.-The commonest type of foliage leaf is the dorsiventral leaf, in which the upper and lower sides are distinctly differentiated. In the first place the stomata are usually present in much greater numbers on, or are even confined to, the lower surface of dorsiventral leaves. Secondly, the mesophyll is differentiated into two strata-the palisade and the spongy tissue. The palisade tissue lies immediately below the upper epidermis. I It consists of from one to several layers of cylindrical or prism-shaped cells with their long axes perpendicular to the surface of the leaf, and with narrow intercellular spaces between them. ${ }^{2}$ The cytoplasm lining the side walls of the palisade cells is packed with chloroplasts (Figs. II, 50), the palisade tissue being the main photosynthetic tissue of the leaf. The spongy tissue occupies the space between the palisade and the lower epidermis, and consists of rounded or irregularly shaped cells with large intercellular spaces between them. Under each stoma there is usually a particularly large intercellular space. The spongy cells contain chloroplasts, but not nearly so many as the palisade cells. The spongy tissue is the main transpiring tissue of the leaf.

The more a plant is exposed to sun and dry air the greater the development of palisade tissue, and in some plants which live in dry sunny climates the whole of the

I Sometimes separated from it by a layer of hypoderm (i்ó, below, $\delta \varepsilon \rho \mu \alpha$, skin), which often acts as a supplementary water tissue, and is often thick walled and helps to strengthen the mechanical structure of the leaf.

${ }_{2}$ The palisade cells are generally in close lateral contact, so that on a cross-section of the leaf no intercellular spaces may be visible. But if a "flat" section parallel to the leaf surface be made through this tissue, the narrow intercellular spaces can always be seen between the cells. 
mesophyll is palisade, thus reducing the rate of transpiration. Leaves held on the plant in a position in which both sides are equally illuminated frequently have the two surfaces alike, palisade being developed under each epidermis. Inversely, in plants growing in damp and shady situations the palisade tissue takes on the characters of spongy tissue, the cells are short and are separated by large intercellular spaces; and in extreme "shade leaves" the whole mesophyll is of the spongy type. The deeper the shade and the damper air (conditions generally found together in nature) the greater the total volume of intercellular spaces in proportion to the total volume of the mesophyll cells.

Water Tissue.-The epidermis of a typical foliage leaf acts, as we have already remarked (p. 275), as a water store for the mesophyll cells ; and, as we have also seen (p. 276), some leaves have a many layered epidermis which greatly increases this water store. In extreme cases the epidermal water tissue may form the greater part of the thickness of the leaf. Part of the mesophyll may also be very poor in, or even destitute of, chloroplasts, e.g. the central tissue of isolateral leaves, and this also acts as a water store for the photosynthetic tissue.

Vascular Bundles (Veins).-Water and salts are brought to the mesophyll of the leaf through the vessels and tracheids of the xylem; while sugars and organic nitrogenous substances are conducted away from the mesophyll through the sieve tubes of the phloem. A strand of xylem and a strand of phloem are associated together to form a vascular bundle, the xylem being towards the upper, the phloem towards the lower face of the leaf. In the leaves of dicotyledons (the greater number of seed plants) there is a large central vein, the midrib, running up the centre of the leaf, and this 
usually projects from the lower surface (sometimes from the upper surface also) and contains several vascular bundles. The outer tissue of the midrib below the epidermis commonly consists of collenchyma (p. 279). From the midrib large secondary veins branch off, and these also are usually thicker than the general thickness of the leaf, so that they project on the lower surface. From the large secondary veins smaller ones branch, and from these smaller ones still, and the branches join again (anastomose), so that the whole substance of the leaf is interpenetrated by a network of vascular bundles. The smaller veins are embedded in the general thickness of the leaf. Each of these smaller bundles is surrounded by a sheath of living cells (bundle sheath), which may or may not contain chloroplasts (Fig. 5o, sh.). The finest branches of the bundle network are destitute of phloem and each ends blindly in the mesophyll as a strand of tracheids covered by the bundle sheath (Fig. 5I, F).

From these terminal tracheids the water coming from the roots through the continuous xylem channels is drawn by osmosis into the mesophyll cells, and the dissolved salts also diffuse, though independently of the water (cf.pp. 290, 296), into the same cells, which are constantly using them up to form complex organic substances. The sugars and amino-compounds, formed as the result of photosynthesis, diffuse out of the mesophyll cells into the bundle sheath, and thence into the sieve tubes, along which they pass into those of the larger veins, and so into the midrib and out of the leaf.

Fibres.-The larger leaf bundles nearly always have a strand of fibres, often crescentic in cross-section, on the outer surface of the phloem, i.e. the surface turned towards the lower face of the leaf, and there is sometimes another similar strand on the upper surface of the xylem, 
i.e. turned towards the upper face. In the larger veins and in the midrib these fibre strands are frequently massive, and they help, sometimes in very notable degree, to stiffen the mechanical structure of the leaf. The veins of the leaf then act rather like the ribs of an umbrella.

The rigidity of the leaf is partly maintained by the turgor of the living cells and partly by the veins, and these two factors vary in their relative importance in different species. ${ }^{I}$ Leaves in which the veins are sufficient by themselves to keep the leaf rigid do not droop even when their living cells are seriously depleted of water. In other cases the base and centre of the leaf is kept rigid by the larger veins, while the less supported tip and edges are drooping. The edges and tip also suffer first from loss of water by evaporation because they are farthest from the source of supply.

Other Forms of Foliage Leaf.-While the great majority of foliage leaves have the form of thin plates, exposing a great surface in proportion to their bulk to the light and air, there are many leaves which differ from this type. Besides the succulent leaves (see p. 275), which may be thick and flat, or oval or even circular in cross-section, the commonest types are the needle-shaped leaves (pine) and the long bristle-shaped leaves of many grasses, especially those which grow in dry places. The narrow leaves of other grasses often fold on the midrib, the stomata being confined to the approximated surfaces, or roll up, in dry weather. These characters all tend to reduce or to protect the transpiring surfaces, and are generally related to diminished water supply or to diminished water-conducting capacity of the xylem.

I A thick and rigid cuticle and a thick-walled hypoderm may also be important in maintaining the rigidity of leaves under severe water loss. This is one reason why evergreen leaves of leathery texture such as those of laurels do not readily droop. 
Sometimes the leaves are small and scale-like, though still green (cypress), and in such cases the stem as well as the leaves may have a well-developed photosynthetic tissue. Finally, the leaves may be reduced to minute functionless scales, photosynthesis and transpiration being carried out entirely by the green stem, to which the photosynthetic tissue, resembling in all respects the mesophyll of a typical leaf, is then confined.

Occasionally in such cases the leaves are represented by bristles or spines instead of scales.

\section{PRACTICAL WORK.}

(I) Make a clean cut under water across the base of the leaf stalk of a geranium (Pelargonium) leaf, and place it in a glass with the cut end dipping below the surface of aqueous eosin solution. After a time observe that the eosin has been sucked up the xylems of the vascular bundles and eventually appears in the leaf veins.

(2) Examine in a drop of dilute glycerine the transverse section of the typical dorsiventral leaf blade (Hellebore) provided. Make a drawing under the high power of $(a)$ the midrib showing xylem, phloem, fibres, collenchyma and epidermis, $(b)$ of a typical segment of the blade showing the upper epidermis with cuticle, palisade cells, spongy tissue, with fewer chloroplasts and large intercellular spaces, and the lower epidermis with a stoma in section.

(3) To another section add a drop of Schulze's solution, and note the different staining of the cuticle and of the wall substance below it.

(4) Strip off a piece of the epidermis from the surface of the leaf of Iris, taking care that you get part of it at least free from adherent mesophyll cells. Mount the strip in dilute glycerine with the outer surface uppermost. Find and draw a stoma with adjacent epidermal cells carefully under the high power. In focussing down, the sides of the rectangular vestibule first come into view, and below this the two guard cells with stomatal pore between them.

(5) Now examine a transverse section of the same leaf. Find and draw very carefully a stoma, which is now seen in sectional view. Note especially the cuticular thickenings of the sides of 
the vestibule and of the walls of the guard cells, and that the latter bulge into the adjacent epidermal cells. Note the intercellular spaces and the mesophyll cells below the stoma.

Examine also in the same section and draw a vascular bundle in cross-section, distinguishing (a) xylem with vessels and tracheids, including the narrow protoxylem elements at the extremity of the bundle, (b) phloem with sieve rubes and companion cells, (c) fibres. Treat with aniline chloride or aniline sulphate. The lignified tissues stain bright yellow.

(6) Cut the end of the leaf stalk of the floating leaf of Limnocharis across with a sharp knife, place the blade in water, and suck the end of the stalk vigorously. Dark patches appear in the leaf blade owing to water being sucked in through the stomata and filling the large intercellular spaces which communicate with those of the leaf stalk.

(7) Examine the shoots of Gorse (Ulex). Note the arrangement. of leaves, branches and buds. The leaves are narrow and spinous, but not so rigid as the branch spines.

(8) Examine any other examples of needle-shaped, bristlelike, folded or rolled leaves that may be available, both on the plants and in section. 


\section{THE PRIMARY STEM}

THE shoot or subaerial part of the body of the higher plant consists, as we have seen, of stem and leaves together. The stem forms the branching axis of the shoot system and bears the leaves, which grow out from its sides. It thus $(a)$ supports and displays the foliage leaves in the light and air, (b) acts as the channel of communication between root and leaves, and $(c)$ supports and displays the flowers, in which the reproductive cells are formed and in which conjugation of the gametes takes place.

Tropisms.-The erect aerial shoot is negatively geotropic, growing away from the centre of the earth, just as the taproot is positively geotropic, growing towards it. This can be seen in plants kept in the dark which grow straight upwards. If the plant is laid on its side in the dark, the actively elongating region of the shoot bends so as to bring the apex into the vertical position again. The shoot is also positively phototropic, growing towards the source of maximum illumination. These two tropisms normally act in the same direction and cause the shoot to grow straight upwards as the opposite ones cause the root to grow downwards. But if illumination is one-sided, the positive phototropism of the shoot overcomes its negative geotropism, so that it bends towards the source of light. This can be seen in the case of many herbaceous plants grown in pots in windows 
which bend so that their shoot apices point towards the open sky, and in plants growing on the edge of a wood which behave in the same way. The leaves tend to set themselves at right angles to the source of light (diaphototropism). As in the case of roots, the bending occurs by the cells on one side of the elongating region growing faster than those on the other, but the apex alone perceives the light, as can be seen by covering the apices of some of a crop of seedlings (e.g. of canary grass) with tinfoil caps and illuminating from one side, when bending no longer takes place in those which are so covered.

Etiolation.-When a normally erect aerial shoot is grown in the dark, it does not turn green, the plastids becoming yellowish, the internodes grow enormously in length and the leaves remain small and scale-like, while the tissues are not properly differentiated. Such a shoot is said to be etiolated.

Structure of the Aerial Stem.-The general structure of the stem depends a good deal upon the fact that it is essentially a leaf-bearing organ. We distinguish the nodes or levels of insertion of the leaves (which may arise from the stem singly or in pairs or circles, called whorls), from the internodes or bare stretches of stem between these levels. The stem terminates in a bud, which is simply the developing apex of the shoot bearing the developing leaves on its sides, and usually covered by the incurving of the partly grown leaves, which arch over and serve to protect the very delicate apical meristem from desiccation.

The minute structure of the outer layers of the stem resembles that of the foliage leaf in more than one respect. Thus it is covered by an epidermis which has the same character as the leaf epidermis, and possesses 
stomata identical with leaf stomata, though they are usually much fewer in number. Below this there is in the green aerial stems of the great majority of plants a living tissue (cortex) containing chlorophyll, though generally not highly specialised like the mesophyll of a foliage leaf. Very frequently, especially in the primary shoots of woody plants, the outer cortex is collenchymatous. In some few cases, especially when the leaves are reduced and functionless, the outer cortex, or portions of it, may be differentiated as palisade tissue.

Below the cortex, as in the root, the vascular cylinder occupies the centre of the stem. But the vascular cylinder of the stem is very different in character from that of the root. In the first place it is generally very much wider (the cortex being correspondingly narrower) in proportion to the whole width of the axis. Secondly, the vascular tissue is arranged like that of the leaf in bundles, each consisting of a strand of xylem and a strand of phloem. Each of these bundles has the xylem towards the centre of the stem, and the phloem outside, towards the cortex; and all the bundles (in the plants called dicotyledons) are arranged side by side, separated by strips of parenchymatous tissue, the rays, and forming a hollow cylinder enclosing the usually parenchymatous pith, which fills up the centre of the stem. Each bundle, like those of the larger leaf veins, nearly always possesses a strand of fibres outside the phloem (Fig. 52, A, B, per.). These fibrous strands may be separated laterally by parenchyma, or they may be joined laterally to form a complete fibrous cylinder. This fibrous cylinder, or the fibrous strands together with the intervening parenchyma, forms the pericycle or outermost layer of the vascular cylinder, and immediately outside it comes the endodermis, whose 
lateral and horizontal walls do not always (as in the root) possess a central band of cutin (see p. 293), though
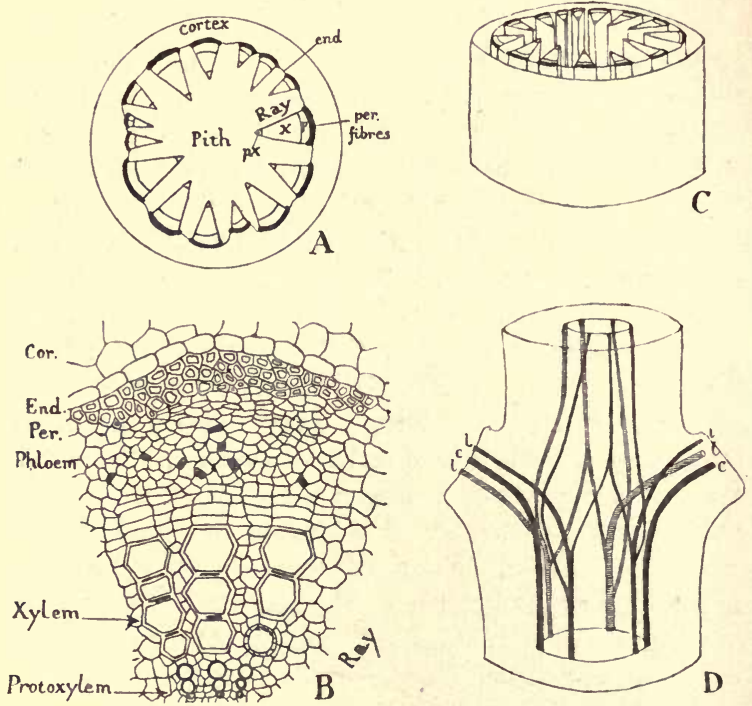

FIG. 52.-Structure of the primary stem. A, diagram of cross-section of stem showing vascular bundles arranged in a cylinder; $X$, xylem; $p x$., protoxylem; $P$, phloem. $B$, single bundle in crosssection ; cor., cortex ; end., endodermis ; per., pericycle (fibrous opposite bundle). C, diagram of segment of stem seen obliquely from above. The cortex and conjunctive tissue of the cylinder are supposed to be transparent, the epidermis and endodermis opaque. $D$, diagram of the course of the leaf trace and stem bundles at a node bearing two opposite leaves in Clematis. The bundles towards the observer are black, those away from the observer shaded. Three bundles enter the stem from each leaf. The laterals $(l)$ at once join bundles from the internode above (two of which fork), the central bundle (c) of each trace continues alone into the internode below (joining with others at a lower node).

sometimes they do. The stem endodermis often differs, however, in other ways from the cells of the cortex 
outside it, e.g. by possessing specially large and conspicuous starch grains, when it is called the "starch sheath."

The vascular cylinder of the stem thus differs in many ways from that of the root, and this is related to its very different conditions of life and to the fact that its bundles are primarily the direct downward continuation of those of the leaves (Fig. 52, D). They are identical in structure with the leaf bundles, and their orientation is the same, the outward position of the phloem in the stem clearly corresponding with its lower position in the leaf.

The stem bundles do not pursue a completely independent course. A certain number come in from each leaf, they often branch, and they always sooner or later join on to neighbouring bundles which have come down through the internode above from higher leaves (Fig. 52, D). Traced downwards through the internode the bundles fuse with one another laterally at various levels, so that they leave room for those entering the cylinder at the next node below. As the base of the stem is approached more fusions take place, so that comparatively few bundles enter the hypocotyl, and these usually fuse with the cotyledon traces, so that only the latter are directly continuous with the vascular cylinder of the root. In the hypocotyl or at the top of the primary root the cylinder narrows, the pith disappears, the pericyclic fibres die out, and the xylem and phloem strands change their relative positions, so that they come to be alternate, i.e. situated on different radial planes. At the same time the cortex changes its character and the shoot epidermis is replaced by the piliferous layer.

Maintenance of the Erect Position.-The herbaceous stem is maintained in an upright position partly by the 
turgidity of its living cells. This is shown by the drooping of many stems, or of their young upper portions, when they are wilted. This source of rigidity is, however, supplemented in many plants by the collenchyma of the outer cortex, which is differentiated early, that is fairly close behind the apical growing point, and stiffens the surface layers of the stem. This surface stiffening resists slight bending strains to which the upper part of the shoot is subjected by the wind. Soon the lignification of the primary xylem elements increases the support afforded by the collenchyma. But the most important mechanical support of most primary herbaceous stems is contributed by the fibres of the pericycle. These are thickened later, i.e. further from the growing point, than the collenchyma, after growth in length has ceased. Owing to the relative narrowness of the cortex compared with the width of the stem, the fibres of the pericycle, which often have very thick walls, form a rigid continuous or interrupted cylinder not far from the surface, and enable the stem to support the weight of leaves and branches and to resist the bending strains imposed by the wind.

Apical Meristem.-A longitudinal section through the tip of the shoot shows the stem tip composed of meristematic (embryonic) cells, and bearing on its sides the first beginnings of the leaves, which arise as projections on the surface caused by locally increased cell division. As the young leaves increase in size they usually grow faster on the lower than on the upper surface, with the result that they curve over the growing tip of the stem with its younger and as yet smaller leaves (Fig. 53), thus serving to protect these and the meristem itself, which are not as yet covered by a well-developed cuticle from drying up and other injury. This curving of the 
partly developed leaves over the shoot tip gives the characteristic " bud" structure.

The surface layer of the meristem is quite separate from the underlying meristematic tissue, i.e. it forms new cell walls only in the direction perpendicular to the surface, and thus gives rise only to the epidermis of the shoot. Below this surface layer of the meristem the cell divisions give rise to the young tissues of the cortex and of the vascular cylinder.

\section{Development of the Shoot immediately behind the} Apex.-As in the case of the root, the products of division of the meristematic cells away from the apex increase in size by the development of vacuoles, and at the same time divide less frequently.

Pith.-The cells in the centre of the stem, which will give rise to the pith, are generally the first to lose their meristematic activity. They often increase greatly in size, partly by their own growth, and partly because they are passively stretched by the active growth of the surrounding tissues. This very frequently results in the pith cells becoming the largest parenchymatous cells in the stem. Sometimes the energetic growth of the surrounding tissues, especially in length, after the growth of the pith cells has ceased, leads to the breaking and ultimate death of the pith. This is the cause of the frequent hollowness of herbaceous stems in which the whole of the pith or its central portion has disappeared in the adult stem.

Zone of the Vascular Bundles, etc.-Just outside the pith, in the zone which gives rise to the vascular bundles, the rays and the pericycle, very many of the cells soon cease to divide by horizontal walls, divisions continuing only parallel to the axis of the stem. Growth in length of these cells continues, so that they become elongated. 
The strands of such elongated cells which will eventually form the vascular bundles are known as the desmogen strands (Fig. 53, desm.), and these soon become distinct from the surrounding tissue. The outlines of the tissues which will be formed later from these often become clear at an early stage of development, and the large cells which will become the vessels of the xylem become apparent. About the same time, or even earlier, the limit between the vascular cylinder and the cortex becomes evident, owing to differentiation between pericycle and cortex, the cells of the latter eontinuing to grow in width.

\section{Development of Leaves and Axillary Buds.-While} these changes have been taking place in the stem tissue, the leaves, which first appear very close to the stem apex as small papillæ, or, where the adult leaves have a broad insertion on the stem, as curved ridges of meristematic tissue, have been growing in length and breadth. In the axil of each leaf there arises, sooner or later, another papilla of meristematic cells, the rudiment of the axillary bud (Fig. 53); and upon the sides of this there arise the rudiments of the first leaves of the lateral shoot into which the bud may develop. The bud may grow out at once to form a branch, not much behind the main shoot in development. On the other hand, it may remain a bud for a long time, even for many years; and in some cases it never develops further at all. Its fate depends on various external and internal conditions.

Differentiation of the Tissues. - The first tissue to attain the adult form is the protoxylem of the vascular bundles. Close to the inner limit of the desmogen strand certain elongated cells become spirally thickened (see p. 280), and the thickening band rapidly becomes lignified, the protoplasm then dying. This happens at first at 
or just below the node of a leaf, in the largest central bundle of the leaf trace, and the differentiation of the protoxylem progresses in both directions, downwards

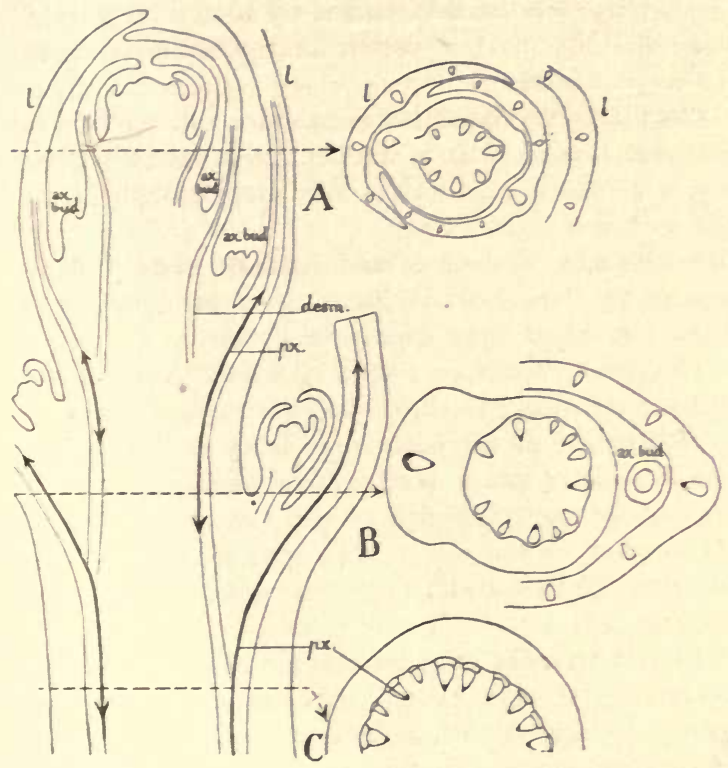

FIG. 53-Diagrams of the differentiation of a stem behind the apex. On the left is a diagram of a longitudinal section, on the right of transverse sections at the levels A, B and C: $l$, leaf; ax.bud., axillary buds; desm., desmogen strand; $p x$. , protoxylem. For further explanation see text.

into the internode below and upwards into the leaf (Fig. 53, $p x$.). In other words, the elongated cells in a line with the first formed spiral tracheids likewise become converted into spiral tracheids, so that a strand of proto- 
xylem is formed which, so to speak, grows in length at each end, extending up into the leaf and down into the stem, and these strands form the first water channels of the shoot. Later, protoxylem is formed in the smaller bundles, and the strands extend till they meet those of other bundles, and thus form a continuous waterconducting system.

Elongating Region of the Stem.-The formation of the protoxylem takes place at the beginning of the elongating region of the stem, which is much longer than that of the root, and often extends over several centimetres or even several inches. It is the internodes which elongate, separating the successive leaves from one another, the nodal regions growing mainly in diameter.

Meanwhile the other tissues of the shoot are rapidly differentiating. The cuticle as it is exposed to the air by elongation of the internodes becomes thicker; the outer layers of the cortex frequently become collenchymatous by the thickening of the longitudinal walls at the corners of the cells; chlorophyll develops in the plastids; and intercellular spaces between many of the cortical cells.

In the vascular cylinder the fibres of the pericycle are clearly marked at an early stage, but their walls are not thickened till relatively late, generally after elongation has ceased. .Narrow sieve tubes (protophloem) usually appear early just below the pericyclic fibres and opposite the protoxylem of each bundle. When growth in length has ceased, the development of the primary vascular bundles continues by the centripetal formation of the larger sieve tubes and companion cells (metaphloem), and of metaxylem, consisting of larger scalariform or some other type of pitted vessels. Differentiation progresses outwards (centrifugally) in the 
primary xylem, the pitted vessels following on the spiral tracheids of the protoxylem. A band of tissue between the xylem and phloem remains undifferentiated, and this is the seat of the cambium or secondary vascular meristem in stems which undergo secondary thickening.

The tissues of the leaf keep pace in differentiation with those of the stem, the leaf blade expanding, while the epidermis, veins and mesophyll acquire their adult characters.

Modified Forms of Shoot.-While the typical aerial shoot stands erect in the air, other forms do not develop the mechanical tissues required to maintain the erect position. Some of these trail on the ground, others twine round any support (such as the erect stem of an upright plant, the side of the stem away from the support growing faster than the side touching it). Other shoots, as we have already seen (Chapter XV), grow below the surface of the soil and do not bear foliage leaves, but only scale leaves. Others, again, grow very little in length but increase in diameter (" rootstocks," corms, tubers). All these types of shoot have the same essential plan of construction as the typical erect aerial stem, but they differ very widely in details. Thus the cortex may be relatively very broad, and it may contain vascular bundles in addition to those of the cylinder In some cases the vascular cylinder early loses its identity altogether, so that the bundles are scattered through the stem. Chlorophyll is not developed in subterranean shoots, nor do they, as a rule, bear stomata. Adventitious roots are practically always produced on subterranean shoots, and very often also on creeping shoots, especially at the nodes, as well as from the bases of erect aerial shoots below the surface of the soil. 


\section{PRACTICAL WORK}

(I) Sketch the piece of herbaceous shoot provided, marking leaf blade, leaf stalk, axillary buds, nodes, internodes. Note and indicate the arrangement of the vascular bundles as seen on the cut surfaces of the stem and leaf stalk: also the epidermis, cortex, pericycle, rays and pith. [A piece of the mature shoot of almost any large herbaceous plant is suitable, e.g. sunflower, vegetable marrow, bean.] Examine also the preparation of a piece of shoot made transparent in canada balsam so that the course of the vascular bundles can be followed.

(2) Pull off the outer leaves of the Brussels sprout provided. This is a bud whose outer leaves become mature with very little elongation of internodes. With a sharp knife or a razor cut a longitudinal section as accurately as possible through the tip, and make a diagrammatic sketch showing (a) growing point (primary meristem), (b) the youngest leaves, (c) older leaves with buds in their axils, (d) young vascular bundles. Pull off the remaining leaves and note their insertions and their axillary buds.

(3) Examine the prepared slide of the same for microscopic details. Note especially the origin of the vascular bundles (desmogen strands), the first spiral tracheids of the protoxylem and the general appearance of the pith cells.

(4) Examine in dilute glycerine a cross-section of the stem of the sunflower, at first with the naked eye and with a hand lens; and then draw a diagram with the help of the low power. Mark the outlines of the following tissues and tissue-systems : epidermis, outer cortex, inner cortex, endodermis (starch sheath); vascular cylinder, consisting of pericycle (composed of fibres opposite the bundles, parenchymatous between), rays, pith, and vascular bundles, showing xylem, phloem and beginning of cambium.

Put a section in a drop of Schulze's solution for a few minutes, and note the colour reactions of the various tissues, showing the distinction between cellulose and lignified tissues.

(5) Now make careful high-power drawings of a few cells of each of the following tissues: (a) epidermis with cuticle; (b) outer cortex (collenchymatous); (c) inner cortex (parenchymatous); (d) endodermis; (e) pericycle fibves; $(f)$ phloem, including sieve tubes, companion cells and parenchyma; $(g)$ cambium; $(h)$ xylem, including large (pitted) vessels, parenchyma and xylem fibres; and (i) protoxylem; (j) cells of the medullary rays which are beginning to form interfascicular cambium.

Examine a prepared stained section of the same to verify details and compare the staining. 
(6) Examine the radial longitudinal section, and draw under the high power a sample of the following tissues: (a) phloem, showing sieve tube (with sieve plate) and companion cell; (b) metaxylem, showing part of a pitted vessel with adjoining parenchyma and fibres; (c) protoxylem, showing spiral vessels and adjoining thin-walled parenchyma. [It is necessary that the section should pass radially through a bundle in order that the above tissues should appear in it.]

(7) Examine the demonstration showing positive phototropism and the fact that the apex of the shoot is the light-perceiving organ [capped Setaria seedlings are suitable]: also examples of etiolated shoots to compare with examples grown in light [bean plants are suitable]. 


\section{CHAPTER XX \\ THE WOODY STEM}

General Characters of Woody Plants.-We saw in Chapter XV that perennial herbaceous plants maintain their vegetative bodies from year to year by means of a persistent underground shoot (rhizome, tuber, corm or bulb), which throws up new aerial shoots at the beginning of the next growing season. New portions of the rhizome are formed every year, new tubers, corms or bulbs are produced, the older parts of the rhizome, the old tubers, corms and bulbs dying off. The aerial shoots of woody plants, on the other hand (trees and shrubs), do not die down every year, leaving only the rhizome or other form of underground shoot to continue growth the next year, but themselves continue growing from year to year, the terminal (or lateral) buds of the branches forming a fresh portion of shoot in each growing season, this being a direct (or indirect) continuation of the portion formed the year before. At the same time the portions of stem formed in earlier years grow in thickness each year, and are thus able both to support and to conduct water and salts from the root to the constantly increasing shoot system above. This process of growth in thickness is called secondary thickening, and is brought about by the activity of the cambium or secondary (vascular) meristem, whose beginnings we have already noted. In most climates, also, there is a definite season of the year unfavourable or impossible for growth (either a 
cold winter or a dry season), and the shoots of a woody perennial must be protected from drying up during that period, and this is effected by another secondary meristem, the cork-cambium.

The main structural features in which a woody perennial differs from a herbaceous plant are therefore three. First, it produces secondary vascular and supporting tissue. Secondly, it has means of protection (bark) of its general shoot surface during the unfavourable season. Thirdly, its aerial buds (winter buds) are protected during the same period.

Winter Buds.-At the tips and on the sides of the younger portions of the branches of a tree or shrub buds are to be seen during the winter covered with brown scales. These bud scales are modified leaves which were formed at the end of the last growing season, and they completely cover in the delicate tissue (apical meristem) in the interior of the bud. The winter bud scales are largely composed of cells with corky (waterproof) walls, and other (gland) cells are often present which secrete a resin or gum that glues the scales together and thus renders the bud additionally watertight. This arrangement not only prevents the tissues within from drying up, but also stops rain from soaking in and rotting the tissues.

Within the bud are young, partly developed foliage leaves, and sometimes, in addition, a group of ready formed flower buds (inflorescence). ${ }^{\mathbf{I}}$ In the case of fruit trees, such as the apple and pear, those winter buds which contain the young flowers are called fruit buds, because, of course, it is the potential production of fruit from the flowers which interests the fruit gardener.

I Compare the buds on the crocus corm and in the tulip bulb, which are also winter buds, though borne on an underground shoot. 
They can be distinguished from the "leaf buds" (buds of shoots which will produce leaves, but no flowers) at any time during the winter in such fruit trees as the pear, and very easily in early spring when they have begun to swell, by their more rounded form.

In the spring the tissue of the axis begins to grow, and this growth eventually bursts the bud and pushes out the foliage leaves, which unfold and grow to their full size, while the internodes of the stem elongate. If there are young flowers in the bud, these also grow and open. The winter bud scales fall off, leaving a zone of scars on the stem, and these will clearly mark the junction of last year's stem and the stem formed during the present growing season. At the close of the growing season the foliage leaves fall off, and the terminal bud (or sometimes a lateral which takes the place of the terminal) once more passes into the winter condition by forming winter bud scales which close it in. These again will fall off and leave another zone of scars in the next spring. In this way successive zones of bud scale scars are left on the stem, and the interval between two of these zones represents a year's growth of the branch. By noting the positions of these zones the history of a woody branch can be read for some years back. Eventually the scars are destroyed by the scaling off of the bark.

Some of the axillary buds formed on the current year's shoot may grow out during the growing season to form branches. The rest form winter buds, and of these some will grow out next spring, but others remain small (dormant buds), and will only grow out later on, under some special stimulus, such as may result, for instance, from the destruction of the terminal shoot.

Secondary Meristems.-Growth in thickness of a 
woody stem is carried on by the activity of a secondary (vascular) meristem (cambium) which arises in the undifferentiated tissue between the xylem and the phloem of each primary bundle, and extends across the rays to form a continuous ring $x$ of meristematic (actively dividing) tissue which runs round the stem. By far the greatest part of the mass of a tree-trunk is formed of secondary xylem, which is the rood of the trunk, by the activity of the cambium.

Another secondary meristem, the cork cambium or phellogen ${ }^{2}$, arises in the stems and roots of most woody plants, external to the vascular tissues. In stems it arises most often in the outermost layer of the cortex, immediately below the epidermis, but sometimes in the epidermis itself, in the pericycle, or in a deep layer of the cortex. This forms the tissue (cork) of which the outer bark of a tree or shrub is largely or wholly composed.

The Cambium and Secondary Thickening.-The cambium consists of active meristematic cells, typically elongated in the direction of the axis of the stem, and also tangentially, while they are often narrow in the radial direction. The top and bottom walls of each cell are commonly inclined like a roof or penthouse, the slopes being directed tangentially (Fig. 54, A). Properly speaking, the term cambium is confined to a single layer of cells, which continually divide by new tangential walls (Fig. 54, A-C), one of the daughter cells at each division becoming a tissue mother cell, giving rise to vascular, fibrous or parenchymatous tissue elements of the secondary xylem or phloem, according to the side on which it is cut off, the other growing again to form the cambial cell. The term is often, however,

I As seen in cross-section : it is really, of course, a cylinder.

2 Greek $\phi \varepsilon \lambda \lambda o ́ s$, cork, and $\gamma \varepsilon v v a ́ \omega$, produce. 
loosely applied not only to the cambial (initial) layer itself, but also to the undifferentiated tissue mother cells on each side of it. When cell division is rapid relatively to the rate of differentiation, there are several layers of this undifferentiated tissue between the fully formed secondary xylem and phloem (Fig. 54, E).

The cambial layer in the bundle itself (interfascicular cambium) is really a layer of the desmogen strand which has remained meristematic, and the process of cell division and differentiation of the products (tissue mother cells) into secondary xylem and phloem elements is a direct continuation of the cell division and differentiation, which has already taken place in the desmogen strand, producing primary xylem and phloem, and proceeding outwards from the protoxylem and inwards from the protophloem. In the case of the xylem especially the process often goes straight on with no pause, the elements of the secondary xylem continuing in the same radial rows as those of the primary xylem, so that it is impossible to say where the one ends and the other begins. In some cases, however, there is a distinct pause between the completion of the primary and the beginning of secondary development.

Each tissue mother cell cut off from a cambial cell on the inside, i.e. towards the primary xylem, may become either $(a)$ a tracheid, (b) a segment of a vessel, (c) a xylem fibre, or $(d)$ by horizontal division a vertical file of parenchyma cells (Fig. 54, D). The cells undergo the corresponding modifications of the cell wall, the protoplasm dying in all except the last case. Each tissue mother cell cut off from a cambial cell on the outside, i.e. towards the primary phloem, may become either (a) a segment of a sieve tube with one or more companion cells, $(b)$ a phloem fibre, or $(c)$ by horizontal 

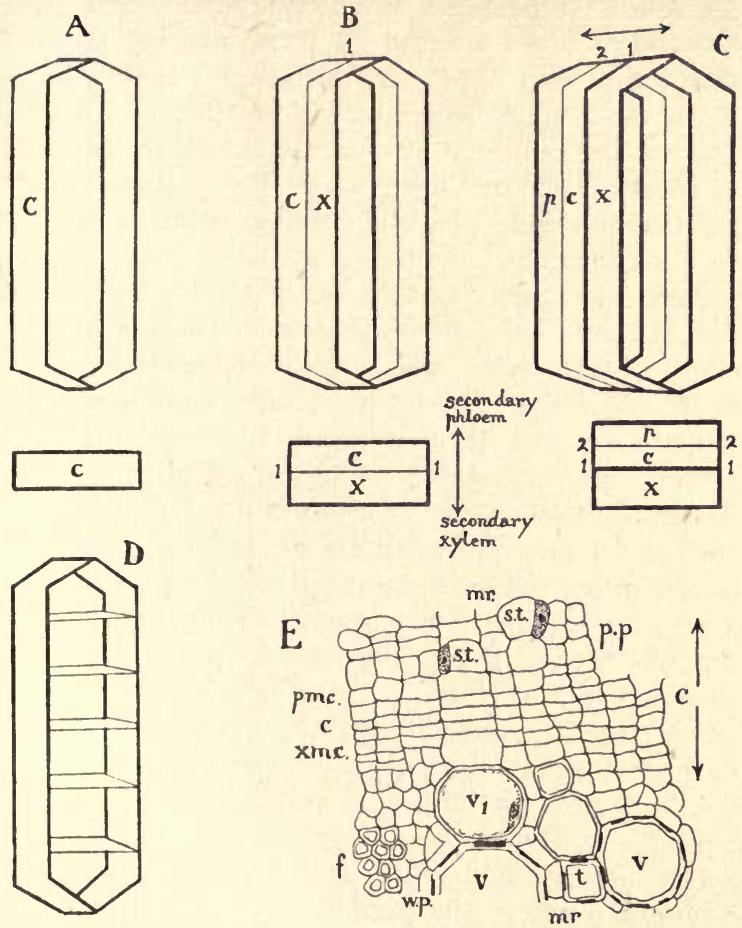

FIG. 54.-Activity of the cambium. A-D, diagrams of cambial cell and its divisions (inner and outer-tangential-walls supposed to be transparent, lateral-radial-walls opaque). A, single cambial cell seen obliquely from within. B, first tangential division (I). C, second tangential division (2); $c$, cambial cell; $x$, xylem tissue mother cell; $p$, phloem tissue mother cell. D, diagram of tissue mother cell which has divided horizontally to form a vertical file of short parenchyma cells. E, part of cambium and tissues it has produced as seen in transverse section under high power; $c$, cambial cells; $v$, xylem vessels; $t$, tracheid ; $m . r .$, medullary ray cells; s.t., young sieve tube; p.p., phloem parenchyma; w.p., wood parenchyma; $f$, fibres. 
division a vertical file of phloem parenchyma cells. The xylem fibres are usually more strongly lignified than the phloem fibres, and the xylem parenchyma is often, though not always, thick walled and lignified. The tracheids and fibres commonly elongate very much in developing from the tissue mother cells, and their pointed ends slide past one another, so as to overlap very considerably.

Secondary Rays.-Certain cambial cells, instead of forming any of the above-mentioned elements, cut off cells on both sides, which then divide horizontally, each cell of the file so formed sometimes elongating radially, so that it corresponds on each side with several elements of the secondary xylem or phloem. Several cambial cells adjacent in the vertical direction (sometimes also several adjacent in the tangential direction) behave simultaneously in this way, and once a set of cambial cells has begun to form them, it does not produce anything but this kind of cell. In this way vertical plates of tissue called secondary rays are formed, running through the secondary vascular tissue in a radial direction, several or many cells deep (i.e. in the vertical direction) and one or several cells broad (i.e. in the tangential direction). As the secondary xylem increases in bulk it also of course increases in circumference, and the cambium increases in circumference with it. This it does by the radial division of its cells, i.e. by putting new cells into its circumference. Every year fresh secondary rays are begun, while those already begun are continued, so that in proceeding from the centre to the circumference of the secondary xylem of a woody stem several years old, the number of rays cut by successively larger circles continually increases, and the secondary tissue, as seen in cross-section, is 
divided up into radial wedges by the rays. The rays are generally joined by tangential bands of xylem parenchyma, so that the wood is divided into relatively small blocks of tracheids and vessels, each in contact with living parenchyma cells. It is probable that this fact is related to the mechanism of conduction of water and other substances through the wood, which is not fully understood. Accompanying the rays are horizontally running intercellular channels which communicate with the cortical system of intercellular spaces, and thus serve to aerate the living cells of the secondary xylem. Broad rays (principal rays) are often formed as continuations of the primary rays (separating the primary bundles) when these are narrow, and can be distinguished from the narrow subordinate rays formed opposite the primary bundles (Fig. 55, C).

Annual Rings. - The well-known concentric rings seen on the cut stump of a felled tree are the expression of the fact that the cambium does not form perfectly uniform wood throughout the year. In the early summer, when its activity begins, it forms numerous and often large vessels with accompanying parenchyma ("spring wood"). These provide new conducting channels for taking water and salts up to the new foliage. In the late summer, after these channels have been provided and the foliage is no longer increasing, the vessels are smaller and fewer in number, and the bulk of the wood produced is often composed of fibres, the water-conducting elements being sometimes quite absent (" autumn wood"). The contrast between the alternating zones of spring and autumn wood gives the rings which can be counted with the'naked eye on the surface of a tree-stump. A check to growth, for instance a severe drought at midsummer or the wholesale destruction of the foliage by caterpillars, 

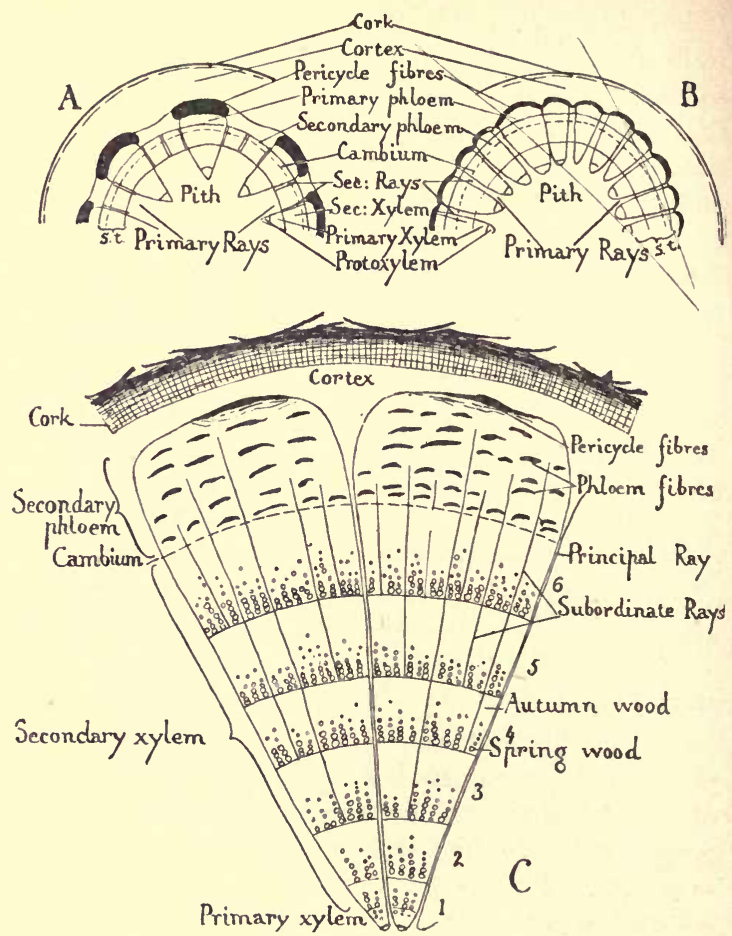

F1G. 55.-Diagrams of secondary thichening in the stem. A, crosssection of stem with bundles separated by broad primary rays in the first year of thickening; secondary xylem and phloem are formed all round the cylinder, bridging the broad primary rays. $B$, ditto, with bundles separated by narrow primary rays; the cambium continues the primary rays as secondary (principal) rays; s.t., secondary tissue. $C$, section of stem after six years of thickening; the primary rays are continued as principal rays, and new narrower subordinate rays are formed in the wedges of secondary tissue formed opposite the primary bundles. 
followed by the outgrowth of buds and the unfolding of fresh leaves, will give rise to a double ring in one year, so that the estimation of the age of a tree by counting the rings is not always quite accurate. Sometimes a similar annual alternation of bands of sieve tubes (spring) and fibres (autumn) can be detected under the microcope in the secondary phloem.

Sapwood and Heartwood.-When a tree-trunk has reached a certain age, which varies in different species of tree, the wood nearest the centre undergoes certain changes, which gradually spread outwards as increase in thickness continues. The living cells (rays and xylem parenchyma) die and the walls of the lignified cells lose water, increase in hardness and usually change in colour, often becoming darker (oak), and sometimes producing a pigment which stains them a distinct colour (e.g. black in ebony, yellow in satinwood and yellowwood, purple in logwood); this harder internal wood is called heartwood. It no longer functions as a waterconducting tissue, and it is the wood most suitable for use in building, furniture making, etc., because it does not warp by losing much water through evaporation on drying. The wood nearest the cambium which is still conducting water and still contains living cells is called sapwood. The sapwood forms a belt of approximately constant width, continually added to on the outside by the cambium, and continually converted into heartwood on the inside.

Some trees have no heartwood, the dead wood in the centre remaining soft and often decaying after it dies, as in the willow. This is frequently the cause of hollow trunks, though hard heartwood too sometimes decays.

Cork Formation.-The phellogen or cork cambium arises in most cases from the outermost layer of cortical 
cells (Fig. $56, a$ ), i.e. immediately below the epidermis, sometimes from the epidermis itself, occasionally in a deeper layer of the cortex, and fairly often in the pericycle. In roots the phellogen is nearly always formed in the pericycle.

Each phellogen cell arises as the result of two rapidly following divisions of the living tissue cell in which it is formed, the two new parallel walls being tangentially

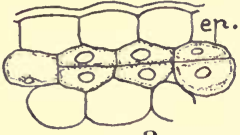

a.

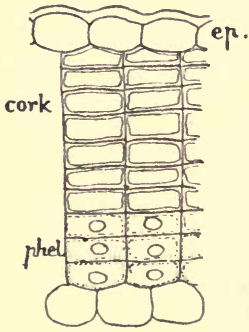

c.
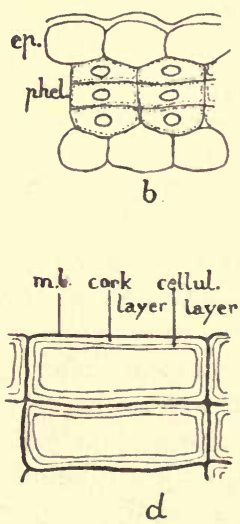

FIG. 56.-Cork formation: $a, b$, Beginning of phellogen in the outermost layer of cortex; ep., epidermis ; phel., phellogen. $c$, Seven layers of cork formed by phellogen below epidermis. $d$, Diagram of two cork cells showing the three layers of the wall (see text).

directed and cutting out the phellogen cell between them (Fig. $56, b, p h e l$. .). The phellogen cell then continues to divide,cutting off a series of daughter cells on the outside, i.e. towards the surface of the stem, forming a regular radial row, and the walls of these are thickened, and become partially converted into corky substance (Fig. $56, c$ ). The middle lamellæ remain unchanged, the next layers 
become corky (a complex substance, suberin, of which fatty acids are constituents, being deposited in them), and a cellulose layer is laid down next the cell cavity (Fig. 56,d). The cork cell then dies, for the corky cell wall is waterproof and prevents the diffusion of water and solutes into and out of the cell necessary to the continued life of the protoplasm. Brown colouring substances are usually formed both in the walls and in the cavities of the cork cells. The thick layer of cork cells with waterproof walls forms a very effective barrier between the living tissues of the plant and the outer world. It is effective not only in checking evaporation and gaseous interchange in general, but also in stopping the attacks of minute insects and the germ tubes of fungal spores. Like the epidermis, however, which it supersedes, it is interrupted at certain spots by openings, and these are called lenticels.

Lenticels are formed in the following way: Cork formation typically begins below the stomata at a somewhat deeper level of the cortex than over the rest of the surface of the stem, and thence it spreads to the outermost cortical layer (or to the epidermis itself) over the general surface. The vigorous cell division below the stomata raises the epidermis at these points and eventually bursts it. The cork cells formed below the stoma are loose, with abundant intercellular spaces between them, and this loose brown tissue is pushed out through the opening made by the bursting of the stoma, and forms a purulent projection on the surface. The whole structure is called a lenticel, and through the air spaces between the cells of the lenticellar cork, which communicate with the intercellular spaces of the cortex below, diffusion of gases takes place between the living cells of the stem and the outside air. 
Bark.-The original phellogen does not, as a rule, remain active indefinitely. In most cases it stops dividing after some years, and a new phellogen is formed in a deeper layer of the cortex, producing a second layer of cork, separated from the first by some layers of cortical cells. This second layer is succeeded by a third, after another interval, at a still deeper level, and so on till the new phellogen come to be formed in the parenchyma cells of the secondary phloem. The later formed phellogens cut deeper and deeper into the secondary phloem, the older parts of which, together with the pericycle and cortex (the whole mass being called the outer bark), are thus killed, being cut off from the living and growing tissues of the stem on each side of the cambium. The zone of functional living phloem (inner bark) thus remains approximately constant in width, being continually reduced on the outside by the formation of deeper and deeper layers of cork, and continually added to on the inside by the cambium. This is a parallel phenomenon to the formation of heartwood from the older secondary xylem. In an old tree-trunk only a comparatively narrow cylinder on each side of the cambium is alive and functional in conduction. The great mass of the tissue of the trunk is dead (heartwood and outer bark), though it may be useful to the tree-the heartwood giving extra support, the outer bark efficient protection.

The bark of trees, as is well known, varies very much in appearance. Some, like the plane tree and the " paper birch," have smooth bark, which scales off in uniform flat thin layers. This is because the phellogens, and consequently the layers of cork which they produce, are uniform and parallel. Others, such as the common oak, the elm and the pine, are rugged, the surface being 
very uneven because the bark scales off in chunks. This is because the courses of the phellogens are irregular and curved, intersecting one another. The actual nature of the cork formed also varies in different trees, sometimes being hard and woody, while in other cases the corky substances of the cell walls is very pure, giving an elastic character to the bark.

Uses of Cork.-The " cork" of ordinary life is obtained from the bark of the cork oak (Quercus suber), an evergreen oak native in the Mediterranean region. The firstformed bark is rough, but after this has been removed the bark subsequently formed is very uniform in structure, and the corky substance of the cell walls very pure. This bark is removed in sheets at intervals of several years, boiled in vats to soften it, pressed flat, and then cut up as required. The dark streaks seen in cork are the lenticles, composed of loose powdery cells, which are continuously formed by the phellogen, so that they penetrate the whole thickness of the bark. Bottle corks are always cut at right angles to the course of the lenticels, i.e. parallel to the surface of the bark, so that solid cork intervenes between the lenticels in the length of the cork. If they were cut the other way so that the lenticels ran through them from end to end, the cork would not be gas and water tight. Bungs, on the other hand, which are not intended to be gastight, are stamped straight out of the cork sheet, so that the lenticels run from top to bottom of the bung.

Cork tissue is not only gas and water tight, it is also a very slow conductor of heat. Thus a jacket of cork prevents the living tissues in the interior of a tree-trunk from being heated or cooled too quickly with external changes of temperature. This character, 
together with its lightness, makes it useful to man for a great variety of purposes.

\section{PRACTICAL WORK.}

(I) Make a sketch of a winter branch of horse-chestnut (or beech) showing several years' growth. Mark terminal buds, lateral buds (distinguishing dormant buds) covered with winter bud scales, leaf scars, bud-scale scars, lenticels; also (in horse-chestnut), if present, the scar of a fallen inflorescence. Mark also the parts of the branch originally formed in each year, beginning with last season and working backwards. Dissect a large terminal bud.

(2) Make a diagrammatic sketch showing the structure of any sprouting bud (sycamore is very suitable, but almost anything will do) after the first foliage leaves are clear of the bud scales, marking winter bud scales, foliage leaves, elongating internodes and terminal bud. [Opening buds preserved in spirit should be provided if fresh material is unavailable owing to the season.]

(3) Examine the transverse section of a woody twig about three years old, first with a hand lens, then with the low power of the microscope. [Sycamore is again suitable, but elm or lime will do equally well.] Draw a diagram under the low power, showing-(a) the cork; (b) the outer cortex; $(c)$ the inner cortex; (d) the pericycle; $(e)$ the secondary phloem; $(f)$ the cambium; $(g)$ the secondary xylem with the annual rings, and in each the spring and autumn wood; (h) the principal secondary rays; (i) the primary xylems; $(j)$ the pith.

(4) Examine the branch of Portugal Laurel that has been kept with its end dipping into red ink, or a watery solution of eosin. Split it longitudinally and note that the red solution has eosin in the wood only.

(5) Make diagrammatic sketches of the three faces (transverse, radial and tangential) of the segment of oak trunk provided, showing-

On the transverse section the annual rings, the large spring vessels which appear as small holes in the wood, the masses of fibres making up the great bulk of the wood, the broad secondary rays crossing the cambium and extending through the functional living phloem of the inner bark (yellow brown) into the dead phloem (red brown) cut off between the dark layers of cork of the outer bark.

On the radial face the spring vessels appear as grooves in the 
wood and the rays as "silver grain," because their course is sinuous. On the tangential face the rays are seen in their lensshaped transverse section, and their vertical extent can be determined.

Note on the transverse and radial faces the distinction between heartwood and sapwood.

(6) Draw under the high power a small portion of a crosssection of the outer tissue of the stem of a woody plant during its first summer's growth to show the origin of the phellogen and the development of cork [Ailanthus is suitable]. Compare the remaining tissues of the stem with (3).

(7) Examine the piece of willow branch provided. Observe the lenticels on the surface. Peel the branch and note that the bark separates along the line of the cambium. 


\section{CHAPTER XXI}

\section{THE FLOWER}

A FLOWER is a shoot, or the termination of a shoot, whose leaves (floral leaves) are specially modified in different ways. The upper (inner) leaves produce the reproductive cells, the lower (outer) ones serve to protect the flower bud and (usually) to render the flower conspicuous. The apical meristem of the flower bud ceases its activity with the production of the floral leaves, so that terminal growth in length of the floral shoot is at an end.

The seed plants are heterosporous, like Selaginella (p. 25I), and while one set of floral leaves bears microsporangia containing microspores, another set bears megasporangia, each megasporangium normally containing one megaspore. The prothallus produced by each is greatly reduced and is formed together with the gametes inside the spore. One of the essential characters of seed plants is the means by which the gametes are brought together. The microspores are carried by external agency, usually insects or the wind, to a special part of the floral leaf bearing the megasporangia. There the microspores germinate, each putting out a germ tube which, carrying the male gametes, grows towards and penetrates the megasporangium, setting free the male gametes inside the megaspore, in the immediate neighbourhood of the female gamete or egg. Conjugation thus takes place inside the megaspore. 
The flowers of some kinds of seed plants are of two kinds, the one producing microsporangia alone (" male" flowers), the other megasporangia alone ("female" flowers); but most seed plants have flowers producing both kinds of sporangia, though on separate floral leaves, and these are called "hermaphrodite" flowers.

Parts of the Flower.-The flower is a condensed shoot in which the internodes have elongated very little, if at all, and the crowded nodes on which the floral leaves are borne are together called the receptacle (Fig. 57). The lower part of the same axis continuous with the receptacle is often bare of leaves, and is called the peduncle if the flowers are borne singly arising from vegetative shoots, the pedicel if it belongs to a system of branches (inflorescence), each bearing a flower. Leaves in the axils of which flowers are borne or themselves borne on peduncle or pedicel and somewhat different from the foliage leaves are called bracts.

Perianth.-The floral leaves themselves are arranged in whorls (a whorl is a circle of leaves arising at the same level on the axis) one above the other. The outer (lowest) leaves form the perianth, which very commonly consists of two whorls, the calyx, whose leaves, the sepals, are often green and rather like small simple foliage leaves, and the corolla, whose leaves, the petals, are generally larger and more or less brightly coloured. Sometimes, however, the perianth consists of one whorl only, or of two whorls whose leaves are alike. In the flower bud the perianth leaves are curved inwards, enclosing the two inner (upper) whorls of floral leaves which bear the two kinds of sporangia.

Stamens and Carpels.-The first (lower) of these is called the androcium, ${ }^{\mathrm{I}}$ the individual leaves the stamens.

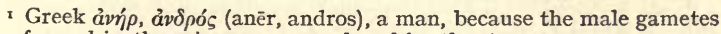
are formed in the microspores produced by the stamens. 
Each stamen has a stalk, the filament, and bears (usually two) pairs of microsporangia, which are known as pollen sacs, together forming the anther or head of the stamen. When ripe the pollen sacs are full

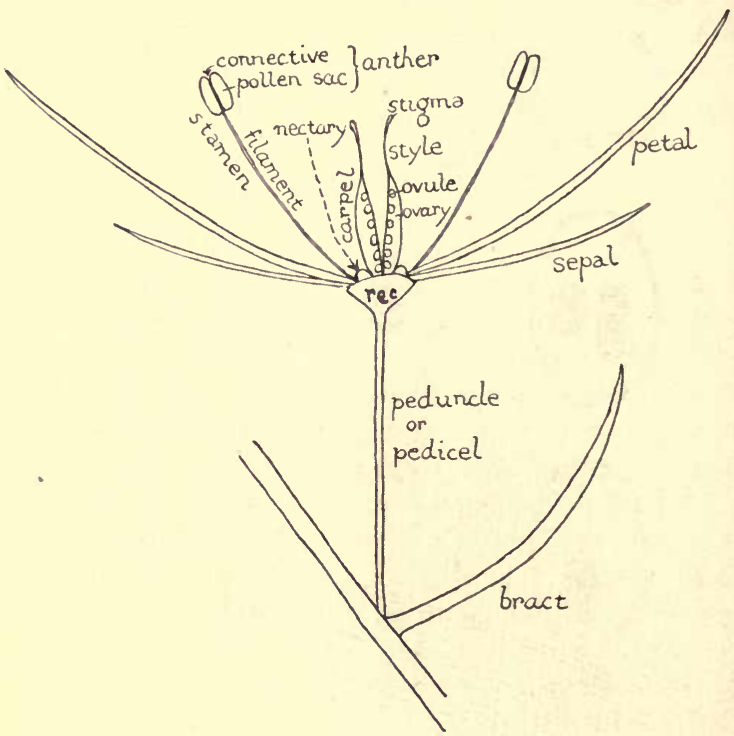

Fig. 57-Diagram showing the parts of a flower.

of pollen grains (microspores) (Fig. 58, A). The uppermost whorl of floral leaves, occupying the centre of the flower, is called the gynæcium, ${ }^{\mathbf{I}}$ and its leaves the carpels. ${ }^{2}$ A carpel is a folded leaf with its margins

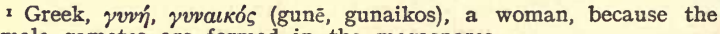
female gametes are formed in the megaspores.

2 Latin carpellum, a little fruit, from Greek $\kappa \alpha \rho \pi o ́ s$, a fruit, because the carpels later on grow into the fruit. 

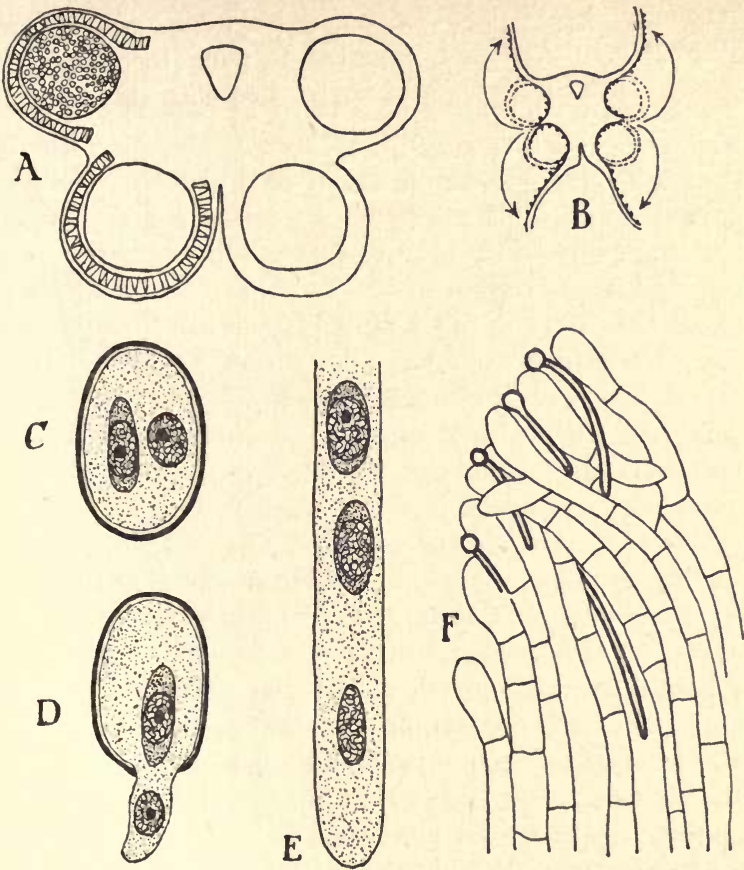

Fig. 58.-Development of pollen (microspores). A, cross-section of anther showing four pollen sacs (microsporangia), one full of pollen grains (microspores); also fibrous layer of wall and vascular bundle. B, cross section of opened anther, with the walls of the pollen sacs turned back. The dotted lines show their original position and the arrows the direction of movement when they break open. C, pollen grain with nucleus (right) and "generation cell," which represents the "male prothallus" (left). D, germination of pollen grain. The nucleus has entered the tube and is followed by the "generative cell." E, growing apex of pollen tube with nucleus and two male gametes into which the generative cell has divided. F, part of stigma with germinating pollen grains, their tubes pushing down between the loose stigmatic cells. 
coherent so as to form a closed bag-like structure, the ovary (Figs. 57, 59, A). The free end of the leaf forms a structure, which, when mature, lacks the characteristic shoot epidermis, bearing on its surface papillæ or hairs which secrete a sugary solution. This is called the stigma (Fig. 57), and is the organ which receives the pollen grains. The stigma is often raised above the ovary on a more or less hollow stalk, the style. On the thickened margins of the carpellary leaf (placenta) inside the cavity of the ovary are the ovules (Fig. 59, A). Each ovule (Fig. 59, B) is a megasporangium covered by two coats, each composed of one or more layers of cells, and with a body (nucellus) consisting of an ovoid mass of cells, of which one, the megaspore, early becomes much larger than the rest, and when mature fills much of the space within the nucellus, one end lying close to the surface of the free end of the nucellus, just below a pore (micropyle) left by the incomplete closure of the coats of the ovule. During development the ovule generally turns completely round upon itself, i.e. through an angle of 180 degrees, so that the free end comes to point towards the placenta on which the ovule is inserted. In a few cases the ovule does not turn, and the free end points away from the placenta.

Development of Gametes.-The pollen grain or microspore when ripe consists of a cell with a thick cutinised outer wall, often covered with projections so that the surface is rough, and a thinner internal wall consisting of cellulose. One or more interruptions in the thick outer wall leave thin places covered only by the thin inner wall. The cell is densely filled with cytoplasm and contains a large conspicuous nucleus. This nucleus divides into two: one daughter nucleus is the nucleus of the ripe grain; the other is somewhat 
elongated and is surrounded by a little cytoplasm, so that it forms a little naked cell within the pollen grain
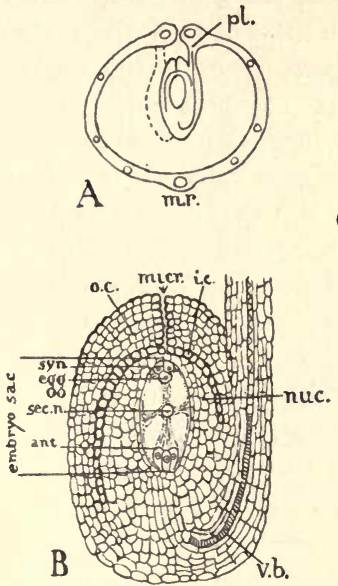
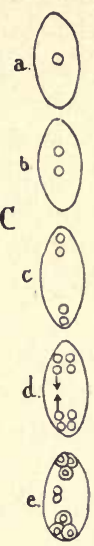

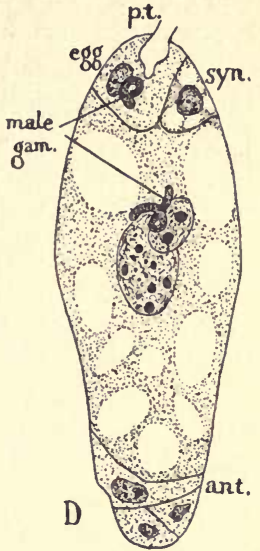

FIG. 59.-The ovule and fertilisation. A, cross-section of a single free carpel containing two rows of ovules; m.r., midrib of carpellary leaf; $p l$., placenta (thickened margin of carpellary leaf) bearing row of ovules. B, ripe ovule (megasporangium) in longitudinal section; v.b., vascular bundle; nuc., nucellus (body of ovule $=$ wall of megasporangium); o.c., outer coat; i.c., inner coat; micr., micropyle. The embryo sac (megaspore) contains in the centre the secondary nucleus (sec.n.); at the micropylar end the two synergidæ (syn.) and the egg, and at the opposite end the three antipodal cells (ant.). C, development of embryo sac; $a-d$, division of primary nucleus to form eight nuclei, four at each end; $e$., formation of egg apparatus, group of antipodal cells and secondary nucleus. D, embryo sac at the time of fertilisation; p.t., pollen tube, which has entered the upper end of the sac and evacuated the two male gametes, one of which is seen in contact with the nucleus of the egg (female gamete) and the other with the two polar nuclei, which in this case have not yet fused to form the secondary nucleus of the sac. Contact will be followed by fusion, in the former case giving the zygote, in the latter the mother nucleus of the endosperm.

cell (Fig. 58, C). This independent cell later divides to form the two male gametes (E). Thus the male 
prothallus is reduced to the lowest possible terms, for it consists of nothing but the single mother cell of the two gametes and the nucleus of the grain which later functions in forming the pollen tube.

The nucleus of the megaspore (embryo sac) divides by three successive divisions to form eight nuclei (Fig. 59, C, $a-e$ ), a group of four at each end of the sac. Two of these nuclei, one from each group of four, fuse together in the centre to form the secondary nucleus of the sac, the other three of each group remain together and accumulate cytoplasm, so that two groups of three cells are formed one at each end of the sac. The three cells at the micropylar end of the sac form the egg apparatus, the cell farthest from the micropyle being the egg (female gamete), the other two the synergids. ${ }^{x}$ The three cells at the opposite end of the sac are called the antipodal cells (Fig. 59, D, ant.).

It is to be noticed that while one cell only of the eight normally functions as a female gamete, its nucleus is the sister of a nucleus which fuses in the centre of the sac with a corresponding nucleus from the antipodal group. The secondary nucleus so formed undergoes, as we shall see presently, a further development. As an abnormality, also, one of the synergidæ or one of the antipodal cells may fuse with a male gamete, thus itself acting as a gamete. We must, therefore, probably regard the two groups of cells as broods of four gametes, only one of the eight being normally functional as a sexual gamete, i.e. fusing with a male gamete, though two others normally behave in a gametelike way. This recalls the brood of eight eggs in

I Synergidæ (Greek $\sigma v^{\prime} v$, with, and $\varepsilon^{\prime} \rho \gamma o v$, work) = "co-operators," because these two cells are supposed to assist in directing the pollen tube to the egg. 
Fucus, of which, it will be remembered, all are functional in some species, while in other species four, two, or only one are functional eggs.

Pollination.-When the pollen is ripe the anthers open in various ways. Very commonly a longitudinal split runs down the side of the anther between the two pollen sacs whose walls break through along this line and fold back, exposing the pollen grains (Fig. 58, B). The split is actually caused by the contraction on drying up of the wall of the sac, whose cell walls have fibrous thickenings, the tension which develops eventually tearing apart the weak unthickened tissue between the two sacs on each side of the anther.

The pollen grains are conveyed to the stigma in various ways. Sometimes the stigma touches the opened anther, rubbing off the pollen (self-pollination); but very often the pollen is conveyed, generally by insects or the wind, to the stigma of another flower of the same species (cross-pollination). The stigma is papillose and sticky, or hairy, so that the grains, which are often rough coated, readily stick to it.

Germination of the Pollen Grains. Fertilisation.The cells of the stigma secrete a sugary solution which is absorbed by the grains lying among them, and these germinate, a tube (pollen tube) being pushed out from the thin spot on the wall. Into this tube pass first the pollen grain nucleus, and then the independent cell, which divides to form the two male gametes during the growth of the tube (Fig. 58, D, E). The tube grows into the stigma (Fig. 58, F) and down through the loose tissue of the style till its tip reaches the cavity of the ovary. The growing tip is positively chemotropic to sugar, or some other substance secreted by the tissue 
in the region of the micropyle, which the tube enters, then pushing between the cells of the nucellus and entering the embryo sac. Here the tip bursts, setting free the two male gametes. These are elongated and often curved or slightly twisted spirally, recalling the spirally twisted male gametes of the lower plants. They appear to wriggle actively in the sac, one fusing with the egg, the other with the secondary nucleus of the sac (Fig. 59, D).

The zygote or "fertilised egg" gives rise to the embryo of the new plant, the secondary nucleus of the sac, to which three nuclei have now contributed, divides to form a new tissue, the endosperm, which fills the sac, and at the expense of which the embryo grows. The endosperm nucleus is of the nature of a zygote nucleus, being formed by the fusion of $(a)$ the sister nucleus of the functional female gamete, $(b)$ a corresponding antipodal nucleus, (c) a male gamete. This " triple fusion" is most unusual, and leads to irregularities in division of the endosperm "zygote" nucleus. The endosperm may thus be regarded as an "abnormally " formed embryo, which is sacrificed to the feeding of the normal embryo.

Varieties of Floral Form and Structure.-The variety of form and structure among flowers is exceedingly great, and seed plants are classified largely by means of these differences. Thus the floral leaves of each kind, particularly the stamens and carpels, vary from a large and indefinite number (buttercup) to a small and definite number (apple, cherry, corncockle). The floral leaves in each whorl may be quite free and separate from one another I (buttercup and Fig. 6o, A) or they

I This is expressed by the prefix apo- (Greek ảmó, away from). Thus the buttercup flower is apocarpous. 
may be joined to one another laterally ${ }^{I}$ (calyx of corncockle, carpels in Fig. 6o, B). The successive whorls of floral leaves may arise from the sides of a conical receptacle (Fig. 6o, A), or they may arise from
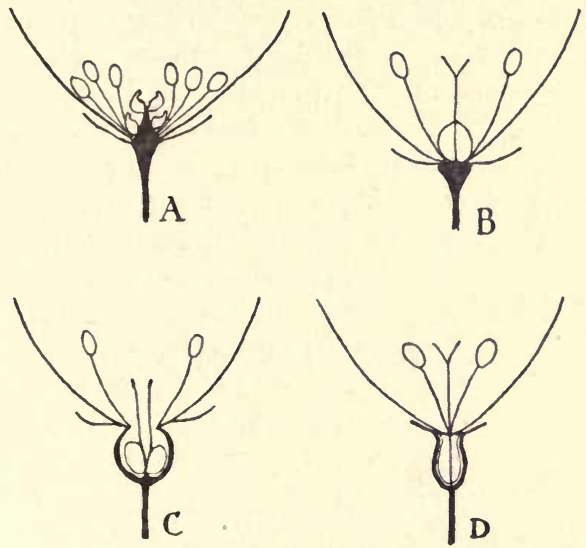

FIG. 60.-Diagrammatic vertical sections illustrating different relations of the receptacle (black throughout) to the floral whorls. A, conical receptacle bearing the successive whorls on its sides (hypogynous type), carpels separate. B, flat receptacle, carpels united (syncarpous gynæcium). C, basin-shaped receptacle with two free carpels at the base, the remaining whorls on the edge of the basin (perigynous type). D, receptacle fused to the sides of the ovaries (epigynous type), which are then said to be "inferior."

a flat receptacle (Fig. 6o, B), or the receptacle may be cup-shaped (Fig. 6o, C, and cherry), with the sepals, petals and stamens arising from the edge of the cup, and the carpels from its bottom. Finally, the sides of

3 Expressed by the prefix syn- (Greek $\sigma \dot{v} v$, together). The corncockle flower is synsepalous. The gynæcium in Fig. 6o, B, is syncarpous. 
the cup-shaped receptacle may be fused with the walls of the carpels (Fig. 6o, D : apple, pear). These different relations of the receptacle to the various whorls of the flower are expressed by the terms hypogynous, perigynous and epigynous, $\mathbf{I}$ the other whorls of the flower being below the gynæcium in the first case, round it (i.e. borne on the edges of the receptacular cup) in the second, and above or on it in the third, where the sides of the receptacle are fused with and close over the walls of the carpels.

On the whole, in the evolution of the great group of flowering plants, the flowers with separate and numerous floral leaves are more primitive, those with few and joined floral leaves the more advanced (the stamens, having thin stalks, generally remain separate throughout, though they are joined to one another in a few cases). And the perigynous and epigynous types of flower are similarly more advanced than the hypogynous. Especially in the case of the carpels, as we pass from more primitive to more advanced flowers, there is a tendency for the carpels $(a)$ to decrease in number, $(b)$ to become fused, and $(c)$ to become enclosed in the receptacle. The ovary is said to be inferior in this last (epigynous) type of flower (apple, narcissus).

Besides these general tendencies in the evolution of flowers there are many other differences depending on the relative size, shape and colour of the different floral leaves. These affect particularly the mode of pollination. A flower has to be regarded as a whole, as an organ which not only produces and protects the spores and gametes but is also so constructed as to bring about the conjugation of the gametes through the preliminary process of pollination. In many cases

× Greek $\dot{v} \pi \dot{r}, \pi \varepsilon \rho l$ and $\dot{\varepsilon} \pi l$, below, round and on. 
the flower is a very wonderful and perfect mechanism for securing this process.

Cross-Pollination by Insects.-A very large number of flowers, probably the great majority, are adapted to cross-pollination by insects. Insects of different kinds (mainly bees, flies, butterflies and moths) visit flowers to feed on the nectar or on the pollen, or on both, or to collect them (bees) for their young. While in the
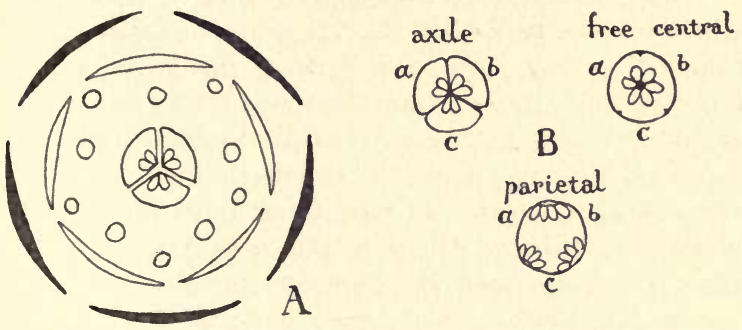

FIG. 61.-A, ground plan of a flower (floral diagram) showing five free sepals (black), five alternating free petals, ten stamens in two alternating whorls of five each, and three free (apocarpous) carpels. B, cross-sections of syncarpous ovaries of three carpels each $(a, b, c)$, showing different types of placentation : axilo (note correspondence of placentre with those of the carpels in A), free central (the infolded carpel walls have disappeared, leaving one central placental column), and parietal (carpels not infolded, margins joined to form placentæ on inside of outer wall).

flower they brush against the ripe anthers, and the pollen grains stick to their hairy bodies. On visiting another flower of the same species-and bees especially often keep to one kind of flower on one journey-the insect may brush against the stigma and rub off the grains. The positions of the ripe anthers and ripe stigmas in the flower are generally such that this probably or even inevitably happens.

The petals of insect-pollinated flowers are often large, brightly coloured and conspicuous, so that the 
larger insects can see them from a distance. The flower often has nectaries, little masses of gland cells which secrete a sugary solution, and this nectar is the main attraction of many flowers to nectar-eating insects. In the case of butterflies and moths it is the sole attraction. The ripe anthers and ripe stigmas are commonly held in such a position that the insect has to brush past them in reaching the nectary.

A very common arrangement is that in which the anthers ripen first, and the filaments bend so as to bring the anthers into the path of the insect, while later on the stigmas ripen and bend into the same position. Such a flower is called protandrous. ${ }^{x}$ An insect visiting the flower in the first stage will carry away pollen, and this will be rubbed on to the stigmas directly it visits a flower in the second stage, since the insect will brush the anthers and stigmas in the two flowers with the same part of its body.

Thus in the buttercup the nectaries are on the inner sides of the petals near their bases. When the petals first open the centre of the flower is occupied by a mass of anthers concealing the undeveloped stigmas. The first anthers to open are those on the outside, next the petals, and the ripening of the anthers progresses gradually inwards to the innermost ones, the ripe anthers bending outwards. This is the "male stage" of the flower. If a large insect visits the flower it alights in the centre on the mass of anthers and pushes its head down between these and the petals to get at the nectar. In doing so pollen will adhere to the lower side of its head or body. By the time the last anthers have opened the carpels have grown up in the middle

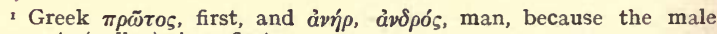
elements (pollen) ripen first. 
of the flower and their stigmas are ripe (incomplete protandry). The same insect alighting on the centre of the flower in this (the "female") stage will brush off on the stigmas any pollen that may be adhering to the lower side of its body from a previous visit to a flower in the male stage, thus effecting cross-pollination. A small insect, on the other hand, may alight anywhere, on the petals or among the anthers, and will crawl down to the nectary or wander about eating pollen, and it will only touch the stigmas in the female stage by chance, if at all. The majority of large conspicuous flowers are pollinated mainly by large insects. In the absence of insect visitors, which do not visit flowers in cold dull weather, the stigmas of the buttercup eventually curl out far enough to come into contact with the innermost anthers, which still probably have some pollen adhering to them, and thus effect self-pollination.

In the corncockle (Lychnis githago) and the pinks (Dianthus) protandry is complete, so that self-pollination is impossible. The anthers lie on the platform provided by the flat limbs of the petals on which the insect must alight. At this time the unripe stigmas are concealed in the narrow tube of the flower. Then the anthers fall off and the stigmas grow up and lie on the platform in exactly the same position that the anthers previously occupied.

In other flowers which are not protandrous the stigmas are generally held well in advance of the anthers, so that they are the first objects the insect meets in entering the flower, and any pollen it already bears will tend to be rubbed off on them. On pushing further into the flower the anthers are encountered.

Some flowers are so nicely adjusted to the structure 
and habits of particular insects that they are only crosspollinated when they receive visits from these insects, and remain sterile, setting no seed, in their absence.

Inconspicuous flowers are often adapted for windpollination (grasses, sedges, most catkin-bearing trees, pines and firs, etc.). Wind-pollinated flowers produce pollen in large quantities, and this is smooth and dustlike, not rough or sticky, like the pollen grains usually conveyed by insects. A great deal is wasted because its carriage to the stigmas of another flower of the same species is a matter of pure chance. The stigmatic surfaces are generally large, and this of course increases the chance of some of the right pollen being caught.

Darwin showed that the offspring of crossing are, on the whole, and with certain exceptions, more vigorous than the offspring of self-pollination, when individuals arising from seed produced in the two ways are grown side by side. Thus variations in the flower tending to secure crossing will tend to be fixed and perpetuated because the offspring of flowers with such variations will tend to survive more often than those in which self-pollination occurred. It seems to be only in this way that we can explain the origin and fixation of the various beautiful and often astonishingly accurate mechanisms which bring about crossing in flowers.

Many inconspicuous flowers (chickweed, the small field speedwells, etc.) are, however, habitually selfpollinated, and these perpetuate themselves indefinitely with perfect success. Many garden and field crops (green peas, wheat) are in the same position. There is no evidence that continued "self-fertilisation" is in any way harmful to the race, though a chance cross in an habitually self-fertilised species will often result in a distinct increase in the vigour of the offspring. 


\section{PRACTICAL WORK.}

(I) Cut a flower of buttercup I (Ranunculus) longitudinally exactly down the middle with a razor or very sharp knife, and draw the cut surface on an enlarged scale, showing $(a)$ receptacle, (b) calyx of sepals, (c) corolla of petals, with (d) nectaries, (e) stamens, (f) carpels.

(2) Draw under a lens a single stamen showing (a) the filament, (b) the anther with two pairs of pollen sacs, (c) the connective (continuation of the filament between the pairs of pollen sacs), (d) the line of dehiscence of the anthers. Draw also a side view of a carpel showing ovary and stigma. Mount a stamen and a carpel in a drop of dilute glycerine on a slide and examine with the low power.

(3) Make a diagram of a section through a mature anther, showing the four pollen sacs, in some of which two nuclei may be seen, the connective traversed by a vascular bundle, and the "fibrous layer" of cells between the epidermis and the cavity of each pollen sac. The cells of this bear rib thickenings on their walls, and it is the tension developed in this layer that splits the anther open.

Compare the section through a dehisced anther.

(4) Remove a carpel from the old flower or young fruit of Caltha (Marsh Marigold), draw the side view showing ovary and stigma, and then split the ovary open so as to show the two rows of ovules attached to the inner edges (towards the centre of the flower), which are the joined margins (placentæ) of the carpellary leaf.

(5) Under a low power draw a diagram of the transverse section of the carpel of Caltha or Aquilegia (Columbine), showing the closed ovary of the carpellary leaf with its midrib, and, on the opposite side, its joined margins (placentæ) to which the ovules are attached. The section should pass longitudinally through the centre of one ovule, and the side of the next belonging to the other row, since the ovules of the two rows alternate.

(6) In a section passing through the centre of an ovule identify and draw carefully under the high power (a) the coats of the ovule (identify if possible the micropyle, which is very narrow and may not be traversed), (b) the nucellus, (c) the embryosac with vacuolated cytoplasm and conspicuous secondary nucleus, $(d)$ the antipodal

I If the buttercup cannot be obtained fresh, it is well to examine first any fairly large fresh flower that can belobtained, draw a median section through it, and then compare with the preserved buttercup flower. 
cells (also very conspicuous), (e) the egg cell and the two synergida. Note that the body of the ovule is turned round so that the micropylar end faces towards the placenta. Note also the vascular bundle running from the placenta up the stalk of the ovule to the base of the nucellus.

(7) Compare the " male" and "female" stages of the corncockle (Lychnis), pink (Dianthus), or other strongly protandrous flower. Make careful drawings of longitudinal sections through the flower in each stage. [In Lychnis or Dianthus show the tubular calyx (synsepalous), the separate petals, each with "claw" and "limb" (the limbs forming the alighting platform for insects), the ten stamens, and the (five) carpels joined (syncarpous) to form a single ovary with free central placenta, and five stigmas.]

(8) Examine a flower of the cherry, and draw a median longitudinal section through it, noting especially the cup-shaped receptacle with sepals, petals and stamens borne on its edge, and the single carpel at the base of the cup, with a long style, which projects above the opening and bears a flat stigma at its summit. 


\section{CHAPTER XXII}

\section{THE FRUIT}

JUST as the development of the flower culminates with the ripening of the spores and the production of the gametes followed by fertilization, so the development of the fruit culminates with the ripening of the seeds, which are the ovules after the zygote (fertilised egg) has developed into the embryo of the new plant.

Development of Seed from Ovule.-After fertilisation the zygote divides and forms a (usually spherical) embryonal cell towards the centre of the embryo sac, and a stalk (suspensor) connecting this with a basal cell, which remains attached to the micropylar end of the sac (Fig. 62, A). The cells of the suspensor divide at right angles to its length and elongate, pushing the embryonal cell down into the endosperm 1 tissue which is formed by the rapid division of the secondary nucleus, food substances being poured into the sac through the vascular bundle. Between the numerous nuclei which arise from this, cell walls appear, thus filling the sac with parenchymatous tissue.

The embryonal cell now divides, and the mass of cells to which it gives rise forms the embryo proper, the suspensor ceasing to grow (Fig. 62, B). In a dicotyledinous plant (the majority of the flowering plants) two rounded projections arise on the free end of the embryo (Fig. 62, C), and these develop into the two

× From Greek $\tilde{\varepsilon} v \delta o v$ and $\sigma \pi \varepsilon \dot{\rho} \mu a$, "inside the seed." 


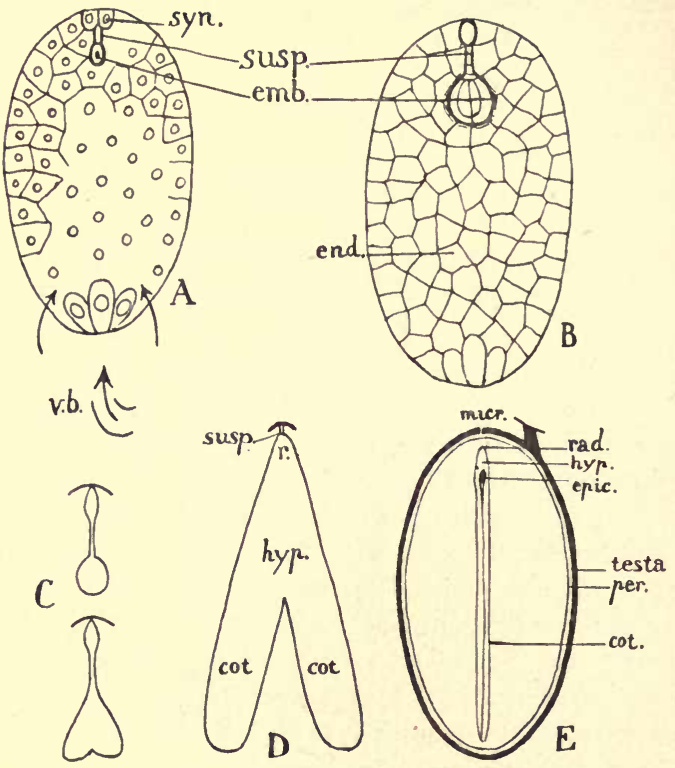

Fig. 62.-Development of embryo. A, embryo sac shortly after fertilisation. The secondary nucleus has divided many times and the sac is rapidly filling with the endosperm tissue; food is coming in through the vascular bundle of the ovule (arrows). The fertilised egg (zygote) has divided to form the suspensor and the embryonal cell. B, sac full of endosperm, into which the embryo is being pushed by the elongation of the suspensor. C, growth of embryo (cells not shown): the free end develops two lobes (the rudiments of the cotyledons). D, older embryo ; cot., cotyledons ; hyp., hypocotyl; $r$, radicle ; susp., suspensor. E, ripe endospermic seed showing embryo with two thin cotyledons closely appressed with epicotyledonary bud (epic.) between, hypocotyl (hyp.), radicle (rad.); also endosperm, perisperm (per.), testa, and micropyle. Note B-E are drawn on progressively smaller scales, the size of the seed and embryo having very greatly increased. 
cotyledons, which are the first two leaves of the plant (Fig. 62, D, E). Between them the terminal bud of the primary shoot axis (epicotyl) is formed. At the other end of the embryo, where it joins the suspensor, the primary root is formed, the apical meristem just inside the surface layer of cells which form the first root cap.

In the monocotyledons $\mathbf{I}$ the free end of the embryo forms the single cotyledon, and the epicotyledonary bud is developed laterally. The part of the primary axis between the cotyledons and the primary root is the hypocotyl.

The embryos of different species vary very much in the stage of development they have reached by the time the seed is ripe. In some cases, particularly in small seeds, the embryo remains in a very rudimentary stage of development, surrounded by the endosperm. In others it develops within the seed, not only large leaf-like cotyledons, but an epicotyledonary bud with the rudiments of several additional leaves. In such large well-developed embryos the vascular system of cotyledons, hypocotyl and primary root is all " blocked out," so that the various tissues can be clearly recognised in the embryo, though the cells remain small and without any thickenings on their walls till germination. Very often the seed grows to many times the size of the ovule at fertilisation, as for instance in the bean. This extensive development of the embryo requires of course an ample supply of food, which is brought up through the vascular bundles of the placenta and of the ovule stalk to the developing seed. The embryo itself grows at the expense of food absorbed from the endo-

I Monocotyledons include the grasses, sedges, lilies, orchids, palms, etc. 
sperm, and this is supplied from the bundle which terminates at the base of the nucellus.

When the embryo is ripe it may fill the whole of the space within the embryo sac, or it may still be surrounded by a mass of endosperm (Fig. 62, E) (see p. 362). The nucellus of the ovule is sometimes represented in the seed by a thin layer of tissue which may be stored with food substance (perisperm). The coat (or coats) of the ovule becomes differentiated in various ways, their cell walls generally thickened and cutinised or lignified, to form the seed coat or testa.

It will be useful here to summarise the corresponding structures in ovule and seed :-

The ovule becomes the seed.

The coat (or coats) of the ovule becomes the testa.

The nucellus may become the perisperm.

The tissue formed by the division of the secondary nucleus of the embryo sac forms the endosperm.

The zygote becomes the embryo (together with the suspensor-a transitory structure).

Development of Fruit from Carpels, etc.-While the changes described above are taking place in the ovule, others are proceeding in the rest of the flower. The petals and stamens usually fall off very soon after fertilisation, while the calyx is often, though not always, persistent, and sometimes grows considerably in size. The stigma and style fall off or wither away, but the walls of the ovary typically develop into the walls of the fruit (pericarp), keeping pace with, or even outstripping, the growth of the seeds. The pericarp, into which the ovary wall develops, differs very much in different species, being sometimes thin and membranous (pea), sometimes thick and woody (hazel nut), sometimes soft and succulent (raspberry, tomato). 
In popular language a fruit is a fleshy envelope which can be eaten enclosing seeds, but the edible part is not necessarily formed from the pericarp. For instance, the fleshy part of an apple, a fig, or a pineapple, is not pericarp at all. The botanical conception of a "true" fruit (i.e. the structure formed from the carpels alone by the time the seeds are ripe) is both wider and narrower than the popular conception. Thus it includes the bean pod and the coconut, while it excludes all but the "pips" of a fig, and the core of an apple. We may, however, conveniently include in the general term. "fruit" all the structures enclosing the ripe seeds, whether derived from pericarp or not.

The only way to understand the nature of the parts of a fruit in this wide sense is to follow their development from the flower. It is especially the receptacle that very frequently takes part in the structure of the fruit. This is necessarily the case in all fruits formed from " inferior" ovaries (p. 354), because the wall of the receptacle is here fused with the wall of the carpels. The wall of the receptacle and the wall of the carpel may together form quite a thin membrane, but, on the other hand, one or other or both may swell up and become fleshy in the fruit.

The cherry, the rose and the apple belong to the same family (Rosaceæ, the rose family), and they illustrate these differences very well. The cherry, as we have already seen, has a cup-shaped receptacle with a single free carpel at the base of the cup. It is the ovary of the carpel alone which forms the cherry. The receptacle does not grow after fertilisation, and is soon flattened out by the great growth of the young fruitit can still be seen as a little disc at the base of the cherry where the stalk joins the fruit. The wall of the carpel 
becomes differentiated into three layers-the "skin," the "flesh" and the "stone" : the "kernel" is the seed.

In the rose the flower is perigynous as in the cherry, but there are many separate carpels, each containing a single seed, within the urn-shaped receptacle, beyond the mouth of which the stigmas project. In the development of the fruit the carpels themselves do not increase very much in size, but the wall of the receptacle grows and becomes fleshy, forming the well-known red " hip " or rose fruit.

In the apple and pear the receptacle in the flower has very much the same shape as in the rose, though it is less elongated; but it is fused with the walls of the five carpels enclosed within it. Both receptacle and ovaries increase greatly in size after fertilisation, the former becoming the "flesh" of the apple and the latter the "core." The "pips" are the seeds. A cross-section of an apple shows that the ovaries are separate from one another as they are in the rose, though they are embedded in the flesh of the receptacle.

In the strawberry the receptacle, instead of being hollow and covering the carpels, is convex, and in the development of the fruit it swells and becomes succulent separating the one-seeded carpels (" pips ") by its great increase in surface, over which the carpels are distributed. The style of each carpel may still be seen attached to each " pip." The cinquefoils, which are close allies of the strawberry, have exactly similar carpels, but the receptacle does not become fleshy, so that the carpels are still crowded together in fruit.

In the mulberry, which is derived from an inflorescence (group of flowers), it is the perianth leaves (two pairs) which become succulent, enclosing the pip-like ovary. 
Sometimes a whole inflorescence develops into a single fruit (often called an "aggregate fruit"). In the fig, for instance, the axis of the inflorescence is concave, bearing the crowded flowers on its inner surface. In fruit the axis (common receptacle of the flowers) becomes succulent, the ovaries ("pips") remaining hard. In the pineapple the whole of the flowers of the inflorescence (including perianth and stamens), as well as the bases of the bracts, become succulent. The hard tips of the bracts are exposed on the surface of this " aggregate" fruit.

Indehiscent and Dehiscent Fruits.-Sometimes the change in form and appearance of the carpels in the passage from flower to fruit is very slight, as in the buttercup, rose, strawberry. In the buttercup, for instance, the carpels, which are one-seeded, scarcely grow at all, but their walls become dry and membranous, changing in colour from green to brown. When ripe they are easily detached from the receptacle and are shaken off by the wind. The pericarp softens and decays in damp soil, and when the seed inside germinates the young plant pushes through its remains. This is called an indehiscent fruit, because the pericarp does not open when the seeds are ripe. A dry one-seeded membranous walled fruit of this kind is called an achene. It is a common type, not only in the buttercup family (Ranunculaceæ), as in buttercup, anemone, clematis, but also in the rose family (Rosaceæ), where it is found in cinquefoil (Potentilla), herb-bennet (Geum) and others ; and it may, as we have seen, be associated with a succulent receptacle (rose, strawberry). Much the same type of fruit is found in the great family Compositæ, in which the flowers are aggregated in close heads (dandelion, thistle), and the outer ones are 
frequently sterile, with a conspicuous one-sided corolla, so that the whole flower-head looks like a single flower (daisy). Here the gynæcium of each flower consists of two united carpels, the ovary being inferior. Only one carpel, however, with one seed, comes to maturity. The wall of the ovary really consists of receptacle and pericarp fused, but since it is membranous and one-seeded the mature fruit is for practical purposes anachene, though it is derived from a bicarpellaryinferior ovary.

A nut is a one-seeded fruit with a woody pericarp. Here again it may be derived from a bicarpellary inferior ovary, as in the common hazel nut (Corylus).

Dry fruits which contain many seeds are practically always dehiscent, i.e. they open to let out the ripe seeds. A simple example is a pea or bean pod. This consists of a single elongated carpel held horizontally in the flower, and generally more or less flattened laterally. The lower edge is the midrib of the carpellary leaf, the upper edge the joined margins (placentæ) bearing the seeds. The seeds and the ovary grow very considerably before ripening, multiplying their original size many times over. When fully grown the walls dry and split along both edges, the seeds being easily detached and falling to the ground. Practically all the pea family (Papilionacex) have this type of fruit (legume). The marsh marigold, hellebore, columbine, larkspur and monkshood-all members of the buttercup family (Ranunculaceæ)-have similar fruits, but they have several separate carpels in each flower, and the fruit splits along the placental margin only (follicle).

Many dry dehiscent fruits are formed from syncarpous gynæcia, with axile, free central or parietal placentation -Fig. 6I (foxglove, corncockle, violet). These all open by 
the longitudinal splitting of the dry capsule, as this kind of fruit is called. In other cases the top of the capsule comes off along a horizontal line (pimpernel). Sometimes the capsule opens by pores in the wall (poppy), out of which the very small seeds are shaken when the stem is swayed by the wind, by which they may be carried a considerable distance from the parent plant.

\section{Methods of Fruit and Seed Dispersal. (I) Dispersal} by Wind.-This is a very common means of dispersal. Seeds and dry one-seeded fruits vary very much in the distance they may be blown. The smaller the seed or fruit the further it will be carried, if the shape and the specific gravity are the same, because the smaller the seed the greater the ratio of surface to bulk. Many small seeds are carried for some distance in this way. On the other hand, a large seed like the broad bean is scarcely affected by the wind and simply drops out of the pod. If a seed is flat instead of being round, its surface will be greatly increased, and it will blow much further. Many seeds and one-seeded fruits bear outgrowths from the testa or pericarp, which greatly increase the surface on which the wind can act. These are of two main kinds-wings and hairs (plumes).

Winged fruits are pretty common. A well-known example is the sycamore fruit. There are two joined carpels in the flower, and from the free side of each a curved wing grows out. When the fruit is ripe the two carpels split apart, and the two are often detached from the tree separately. The shape of the wing causes the one-seeded " mericarp" (separated part of the whole fruit) to spin as it falls, and it is thus more likely to be carried away by the wind. Other examples of winged one-seeded fruits are those of the ash (" keys"), the birch, and the hornbeam. In the last two 
the wings are formed by adherent bracts. Examples of winged seeds are those of the pine and of the tropical genus Zanonia.

Examples of plumed fruits are those of the " old man's beard" (the wild clematis), where the styles are persistent in the fruit and are clothed with long silky hairs, and those of the dandelion, groundsel, thistle ("thistle down"), and of many other members of the Compositæ. Here there is a special plumed structure, the pappus, surmounting the indehiscent one-seeded fruit. Examples of plumed seeds are those of the willows, and of the various species of willowherb (the riverside " codlins and cream," the " rosebay " willowherb of open woods, and the other species). Here the capsule opens and sets free the crowds of tiny seeds which drift about, floated in the air by their very fine silky hairs.

(2) Dispersal by Animals.-Some indehiscent fruits bear hooks which catch on passing animals and stick to their coats, the fruits being readily pulled off the plant. Brushed off later on, the seed may germinate at a distance from the parent plant. The hooks are sometimes outgrowths of the pericarp ("cleavers," Galium aparine), or the hook is formed from the style (herb-bennet, Geum urbanum). In the burdock (Arctium), a member of the Compositæ, the bracts surrounding the flower head are hooked, and the head when in fruit is easily detached, so that it is carried off as a whole, and the dry one-seeded fruits drop off. All these fruits very readily stick to the trousers or skirt as the wearer brushes against the plants.

Animals are also constantly distributing dry fruits and seeds even if these have no special means of adhering to them-carrying them about in the crevices of their 
bodies, in the mud adhering to their feet and so on. An almost incredible number of different seeds and small dry fruits may often be found in the pockets and seams of the clothes as well as on the boots of men who spend much time in the open country.

In the case of succulent fruits which are sought and eaten by birds and mammals, the seeds, which are protected by an indigestible covering, are often swallowed and voided at a distance. The attractive succulent part of the fruit may be the inner layers of the pericarp (melon, raspberry, date, etc.) or special ingrowths from the pericarp (orange), or it may be the perianth leaves (mulberry), or the receptacle of the flower which has become fleshy in the fruit (strawberry, apple), or of the whole inflorescence. The seeds of such fruits are nearly always protected by a layer of hard-walled cells, which resist the digestive enzymes secreted in the alimentary canal of the animal and thus prevent the substance of the seed from being digested. In most cases this is the testa of the seed, but when the succulent part of the fruit is the receptacle or perianth, it is the whole pericarp (strawberry, apple, fig, mulberry), or where the outer layers of the pericarp alone are succulent, it is the inner layer, i.e. the "stone" of "stone fruits," the seed itself (the kernel) being digestible once the stone is removed.

Very often the seeds of succulent fruits stick to the beaks of birds pecking at the fruit, because they are surrounded by a sticky pulp, and are later rubbed off or fall off when the pulp dries. The seeds of mistletoe are distributed in this way. The birds peck at the mistletoe berries and the seeds stick to their bills. They clean their bills by rubbing them on the bark of

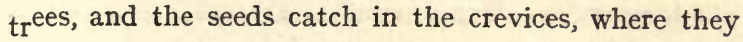


germinate. The mistletoe is a parasite on trees, and the modified roots (haustoria) produced by the seedling penetrate the bark and into the wood of the tree.

(3) Dispersal by Water Carriage.-Many seeds and fruits float when they drop into water, and may thus be carried considerable distances by rivers, germinating if they are stranded in a suitable spot. Many seeds become waterlogged and sink after a short time, but others float for long periods, or indefinitely. The seeds of many riverside plants are surrounded by a tissue containing much air, and are thus specially equipped for floating.

The seeds and fruits of a large number of species of tropical seashore plants-both trees and herbs-have such a floating tissue and are able to stand long soaking in salt water without harm. They are constantly carried about in shore currents and stranded on the coast, where they germinate and produce fresh vegetation. Newly formed islands of coral or volcanic material are largely populated by plants in this way, and this feature of tropical coast plants is also largely responsible for the great uniformity of this vegetation over very wide areas. The coconut is the best-known example, the very thick woody pericarp being very light in proportion to its bulk and causing the fruit to float indefinitely.

It has been shown that this character of coastal plants has been developed in different species from other causes not related to the capacity for floating. But it is clear that the species whose seeds and fruits can float will have a much greater chance of successful propagation, and especially of wide dispersal, than if they had seeds or fruits which sank at once or could not long resist soaking in salt water. There is thus a 
strong selective effect which will inevitably lead to the prevalence of these species in this particular habitat.

The same general consideration holds of most of these so-called " adaptive" characters. They have not in the first instance appeared in relation to the conditions in which they are of special use to the organism, but the existence of the character may favour the spread of the species when these conditions arise, and may even in extreme cases secure its continued existence in circumstances which would lead to the extinction of a species not so equipped.

\section{PRACTICAL WORK.}

\section{Development of Seed and Embryo.}

(I) Slit open the fresh ovaries of Shepherd's Purse (Capsella), choosing those from which the petals have just fallen, and older stages up to the full-sized capsules. Draw under the low power seeds of different stages of development.

Squeeze the ovules of different ages under a coverslip so as to press out the embryos, and draw various stages in the development of the embryo. Note the suspensor and the spherical embryo in the young stage, and the subsequent development of the two cotyledons.

\section{Dehiscent Fruits.}

(2) Examine the young fruit of the Sweet Pea, (Lathyrus) projecting from the remains of the flower. Split the ovary down and note the two rows of young seeds attached to the upper edge. Note the similarity of the single carpel here with the carpel of Caltha (p. 359). In the ripe fruit note the mature seeds and the mode of dehiscence.

(3) Examine any available examples of syncarpous, dry dehiscent fruits (capsules).

\section{Indehiscent Dry One-Seeded Fruits.}

(4) Cut the young " aggregate fruits" of Herb-bennet (Geum) longitudinally in half so as to show the insertion of the individual achenes on the receptacle. Note that a hook is developed in the style just below the stigma, which falls off. 
(5) Draw a single fruit of Dandelion (Tavaxacum), showing the stalked pappus surmounting the one-seeded, dry indehiscent ovary.

(6) Draw the fruit of sycamore (Acer), consisting of two dry one-seeded carpels. The pericarp of each has grown out in the flower to form a long curved wing. Note that the two carpels easily separate. Dissect one of the seeds, and note the thin testa and the green coiled embryo with long strap-shaped cotyledons.

Examine the flower of the sycamore and note the developing wings of the ovary, and that each carpel originally contained two ovules.

\section{Succulent Fruits.}

(7) In the young cherry (Prunus) note the suture (joined margins) of the single carpel, the scar where the style has fallen off, and the flattened disc-like (once basin-shaped) receptacle. Cut a longitudinal section, and distinguish the three layers of the pericarp (skin, flesh and stone). In the young seed distinguish the testa nucellus and gelatinous endosperm. Look for the young embryo at one end.

Compare the ripe cherry. Break the stone and dissect the seed (kernel). The embryo with two cotyledons now occupies the whole space.

(8) Draw a median longitudinal section of an Apple or Pear flower (Pyrus) from which the petals have fallen and in which the receptacle is distinctly swollen. Note the tubular receptacle, with the sepals attached to its rim, and the remains of the stamens just inside it. The ovaries of the carpels are buried at the base of the tube, and the styles pass up through the centre.

(9) Draw transverse and longitudinal sections of a partly grown apple or pear (about three-quarters of an inch in diameter) showing the relation of the carpels (core) to the fleshy receptacle.

(Io) Draw similar sections of a ripe apple or pear, identifying the structures visible with those seen in the earlier stages. Note the withered styles still lying in the central tube, the remains of the stamen still often present within the sepals : the vascular cylinder running from the top of the stalk to the base of the carpels and its continuation through the central column serving the placentæ; also the bundles running through the flesh of the receptacle. 


\section{THE SEED AND ITS GERMINATION}

We have already seen (p. 363) that different seeds vary very much in the size attained by the embryo when the seed is ripe, apart from the dimensions of the seed itself, which varies from the microscopic seeds of orchids to the enormous seed of the coconut. During development the embryo absorbs more or less of the endosperm, but in some cases it is still surrounded by endosperm in the ripe seed, while in others it absorbs the whole of the endosperm and comes to occupy the entire space within the testa. Seeds in which endosperm is still present at maturity are called endospermic seeds, ${ }^{\mathbf{x}}$ and those which have no endosperm left when they are ripe are non-endospermic. ${ }^{2}$ Examples of the former are wheat, maize (and the cereals generally), castor oil, date, etc. : of the latter, bean, pea, acorn, etc. In non-endospermic seeds the embryo, and especially the cotyledons, are often large and swollen, their cells packed with the food material which in the endospermic seeds would remain in the endosperm till the time of germination. This food material takes the forms with which we have already become familiar. The nitrogenous organic substance is in the form of solid grains of protein material, the non-nitrogenous, either as carbohydratestarch grains, or more rarely as thick cellulose walls

"The old name is " albuminous seeds," from the analogy of the "white" or "albumen" of a bird's egg, which, like the endosperm, is a store of food for the developing embryo.

"Or " exalbuminous." 
e.g. date) - or as fats, in the form of oil drops, plant fats being liquid at ordinary temperatures: the cytoplasm of the cells of the endosperm (and perisperm if present) or of the embryo itself being densely packed with these substances. The proportion of water in a seed is very much less than in the actively growing vegetative parts of the plant.

Conditions of Germination.-So long as the ripe seed is kept dry the protoplasm of its cells remains in a dormant condition. Seeds may remain alive in this condition for many years, and though the germination of "mummy wheat" has not been verified, such an occurrence is not by any means out of the question. Seeds have been known to germinate after keeping dry for a century, though some seeds die within a comparatively short time.

The first requisite for germination is liquid water Some ripe seeds (e.g. acorns) contain enough water to germinate at once if evaporation is checked by keeping them in saturated air, but once they have lost a certain proportion of their water they must have more supplied to them from outside in order to germinate. The second requisite is free oxygen. The living cells of the embryo must be able to respire in order to liberate the energy necessary for growth; and both water and oxygen are necessary to the chemical changes which precede and accompany germination. The third is a suitable temperature. At or below $0^{\circ} \mathrm{C}$. protoplasm is inactive, and germination cannot occur any more than can the growth processes of an ordinary growing plant. Above this temperature the chemical changes on which germination depends takes place more and more quickly, so that the higher the temperature, up to a certain point, the more rapid germination will be. But above a 
certain temperature the working of the protoplasmic "machine" is thrown out of gear and death results.

Enzyme Formation and Activity.-One of the first processes preparatory to germination is the production of enzymes in the cells of the endosperm and embryo, by the activity of which the various reserve foods are converted into soluble forms. The starch is acted upon by diastase, the cellulose (as in the date seed) by cytase, the fats by the lipases, the proteins by various proteases (see p. 45). The carbohydrates and fats thus give rise to great quantities of sugars, and these are largely used for respiration, i.e. for the liberation of energy which is seen in the active growth of the embryo: part of this energy takes the form of heat. Thus the main interchanges between the germinating seed and its surroundings are first of all the active absorption of water resulting in the swelling of the seed (cf. the experiment with peas, p. 6o), then the absorption of free oxygen, and the evolution of large quantities of carbon dioxide and heat (p. 89). Some of the sugar is used for forming new cell walls, while the soluble nitrogenous substances into which the solid proteins are converted by the activity of proteolytic enzymes are worked up again to form the basis of the new protoplasm produced in active cell division.

Structural Changes in Germination. Epigeal and Hypogeal Cotyledons.-Most of the cells of the embryo, especially if it is small and relatively slightly developed in the resting stage, actively divide during the first stage of growth, but cell division soon becomes mainly concentrated in the apical meristems. The actual growth of the embryo into the seedling takes place very largely by vacuolation and consequent increase in size of the cells. 
The first external change is the pushing out of the radicle into the soil, sometimes through the micropyle, sometimes through a break in the testa, into the soil, where it bends straight downwards to become the taproot, and often grows to a considerable length before the shoot appears. In plants with a "fibrous" root system the development of the taproot soon stops, and other roots grow out from the base.

In the development of the shoot we have to distinguish two types of germination. In the first, by far the commonest type, the hypocotyl increases enormously in length, thrusting, or sometimes pulling, the cotyledons, with the epicotyledonary bud lying between them, out of the testa (Fig. 63, A). The cotyledons are said to be epigeal I (raised above the soil). The cotyledons are often held with a certain firmness by the testa, and the base of the hypocotyl being fixed by the primary root in the soil, the hypocotyl curves in elongating, setting up a strain and eventually hauling the cotyledons out of the testa. As soon as these are free the hypocotyl straightens out, carrying the cotyledons on its summit, and these expand and turn green (if they are not green already), standing out on each side in a horizontal or upwardly inclined position.

This is the end of the first stage of growth of the seedling, which thus becomes an autonomous green plant, no longer dependent on the food in the endosperm, which has been mostly absorbed by the time the hypocotyl elongates. Many epigeal cotyledons are thin, like the later formed foliage leaves, but in some cases the fleshy cotyledons of non-endospermic seeds are raised above the ground (French bean).

Sometimes the cotyledons are not at once successfully

× Greek $\dot{\varepsilon} \pi \hat{i}$, on, and $\gamma \bar{\eta}$, earth. 
freed from the testa, which is carried up, sticking on to one or both. In most cases it is soon shaken off by their further growth, but occasionally seedlings are

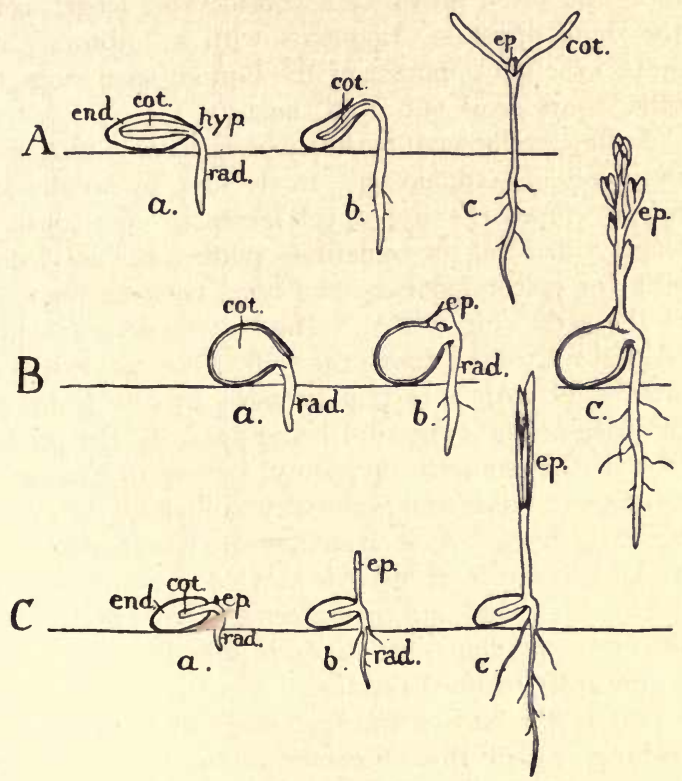

FIG. 63.-Types of germination. A, endospermic seed with epigeal cotyledons. B, non-endospermic seed with hypogeal cotyledons. C, endospermic seed with hypogeal cotyledons, which remains embedded in the endosperm and acts as a sucker ; end., endosperm; cot., cotyledon; ep., epicotyl; rad., radicle.

handicapped, or even killed from this cause. In the marrow a projection of tissue is developed from the base of the hypocotyl which holds down one side of the testa (the seed being here flattened and the testa 
consisting of two valves which grip the cotyledons rather like the two shells of an oyster) and acts as a fulcrum on which the elongating hypocotyl works in pulling the cotyledons out of the testa.

In the second type of germination the cotyledons remain within the seed (hypogeal $\mathbf{x}$ ). The hypocotyl does not elongate, but the epicotyledonary bud at once grows out, at the expense of the food stored in the cotyledons, when these are large and swollen as in non-endospermic seeds (Fig. 63, B), (bean, acorn); while in endospermic seeds the cotyledon acts as a "sucker," remaining embedded in the endosperm, absorbing and transmitting the soluble foodstuff from the endosperm to the growing parts of the seedling (Fig. 63, C). This is well seen in the monocotyledonous date seed in which the peg-like cotyledon embedded in the endosperm produces cytase, which attacks the solid cellulose of the endosperm and absorbs the sugar produced. In the cereals a special organ, the scutellum, lies between the axis of the embryo and the endosperm, and its surface cells secrete diastase which passes into the starchy endosperm, attacks and hydrolyses the starch grains, the sugar produced being absorbed by the scutellum. In all cases the endosperm or cotyledon cells contain protein grains, the nitrogenous material represented by which is of vital importance in the formation of new protoplasm, though much less in amount than the carbohydrate material. Much of the sugar derived from this latter is broken up in the intense respiration which occurs in the germinating seed.

The plant may in fact be regarded as living at its highest intensity during germination. At no other time is there so extensive and rapid a mobilisation of food s $\pi \delta$ and $\gamma \tilde{\eta}$, below the earth. 
reserves for purposes of growth, whether on the side of the formation of new protoplasm and cell walls, or on the side of the expenditure of energy. A comparable situation occurs at the resumption of growth in a perennial plant after the winter rest, when the winter buds on the rhizome or woody stem grow out to form new shoots; but it is not so intense or concentrated as in the seedling.

Use of Seeds by Man.-Owing to the concentrated supplies of organic food they contain seeds form by far the most important vegetable foodstuffs of man. The great bulk of his cultivated crops are grown for the seed. First in importance among these come the cereals : wheat, rice, maize, millet, barley, oats and rye, which all belong to the great family of grasses (Gramineæ). These are all starchy seeds, but with a considerable proportion of protein. The protein varies in different cereals, for instance wheat, the standard food of the white man, contains considerably more than rice, the standard food of the natives of large parts of the tropics. Barley is grown in central and north Europe mainly for malting, the seeds being germinated (malt) and the reserves rendered soluble, so that they can be dissolved out in hot water to form the basis (wort) for the making of beer (p. I32). But in other parts of the world barley is grown for bread-making.

Next in importance to the cereals come the pulses (beans, peas, lentils, etc.), which belong to the legume family, the fruit consisting of a single dehiscent carpel (p. 368). Most of these are also rich in starch, but contain a particularly high proportion of proteins. The pulses are also widely used as foodstuffs, both for men and cattle, but not so extensively as the cereals. Finally, there are the fat (oil) containing seeds. 
Of these certain species of palm are among the chief. The West African oil-palm (Elais guineensis) and the coconut palm (Cocos nucifera) are the two leading species. The former is a constituent of most of the native food in West Africa, and it has been extensively planted in other parts of the continent. In comparatively recent years a great export trade in " palm kernels" has been developed, and from these the oil is extracted for various purposes, prominent among which is the manufacture of margarine. The coconut is used very extensively by the natives throughout the damp tropics for food, lighting and other things, and the oily endosperm is very extensively sun-dried and exported as copra, which is used for margarine, soap-making, etc.

Other important oil-bearing seeds are the " soy bean " (a leguminous plant), linseed (the seed of the flax), and cottonseed (the seed of the cotton plant), the oils of which are all used in making food and for a variety of industrial purposes. After most of the oil is extracted the residue is utilised as cattle food (" cake ").

Relation of the Size of the Seed to Dispersal and to the Chances of Life of the Seedling.-A large seed containing a large amount of food material naturally enables a large seedling to be produced in a comparatively short time, just as a considerable shoot system can be quickly produced from a bulb or tuber: whereas a seedling produced from a small seed uses up its reserves very quickly, and has the laborious work of slowly building up new organic substance from inorganic, with a root and leaf surface which is at first of very limited extent. Provided it finds a suitable spot for germination and growth, the seedling derived from a large seed thus has a great initial advantage in competing with other plants for space and light, because it is less likely to be 
smothered and to have its light cut off by the vegetation around it

Plants producing small seeds, on the other hand, have two great advantages. The seeds can be produced in far greater numbers with an equal supply of food from the parent plant, and they are much more readily dispersed, so that there is more chance of many of them reaching comparatively distant spots where they can germinate and establish themselves, and thus the species has a better chance of wide distribution and ultimate survival. The two most widely distributed and numerous families of flowering plants, the grasses (Gramineæ) and the composites (Compositæ), both have small one-seeded fruits, and distribution is often facilitated in the latter case by the pappus (p. 370).

Death Rate and Competition.-The death rate of seeds and seedlings in nature, like that of all young organisms, is enormous. Besides the large number of seeds which fall in places where they cannot germinate and the large number that are eaten by animals, many seeds which do begin to germinate are killed at an early stage by finding no suitable soil in which they can root, by being smothered or cut off from light by other plants, or by the attacks of fungi or small insects. At a rather later stage very many are eaten off by rodents or by browsing animals. No seedling of a woody plant can survive, for instance, in heavily pastured grassland. Perennial herbaceous plants like the grasses survive in such land because of their underground and surface shoot systems, which possess buds that grow out as soon as the upper shoots are eaten off.

If we suppose an annual plant to produce only ten seedlings a year, and all of these survive and themselves produce seed, we should have in the twelfth 
year a million million individuals of that species, the offspring, in the twelfth generation, of a single plant. Many species at the present time are actively spreading and increasing their territory, though the increase is never more than a minute fraction of that which would occur if all offspring survived. Many others are decreasing, not holding their own in the struggle for space, light and water. Probably a much larger number of species are more or less holding their own. It is by competition between individuals of the same and different species that this approximate balance is maintained.

\section{Significance of the Seed in the Evolution of the Plant} World.-The ovule, and the seed into which the ovule develops, represent the culmination of the adaptation of the sexual reproductive processes to the conditions of plant life on land, where an external supply of water on the surface of the soil is no longer available. We saw (in Chapter XIV) the preparatory stages which necessarily preceded the appearance of seeds, and which developed many millions of years ago, during the Palæozoic age, in the ancestors of our modern seed plants. These preparatory stages are still represented in the few existing heterosporous Pteridophytes. Instead of uniform spores which germinate to form green freeliving plants - the prothalli of such plants as fernswhich produce the sexual organs, the heterosporous forms produce two kinds of spores, the large megaspores and the small microspores, which germinate to form microscopic prothalli, dependent on the spores, and respectively producing the male and the female gametes. The process of conjugation still depends, however, on external liquid water in which the flagellate male gametes can swim, and this dependence severely restricts the habitats in which such plants can live. 
The progress to the development of the ovule depends on the retention of the megaspore within the megasporangium and the appearance of some means other than their own locomotion in water of bringing the male gametes to the female. We do not know all the steps in the evolutionary process, because we have not discovered the fossil forms which were the actual ancestors of our modern seed plants. But the essential new features are two, first the appearance of a wall of cells to the megasporangium (nucellus of the ovule) and of one or two coats of cells covering the nucellus, and secondly of the germination of the microspore (pollen grain) to form a germ tube (pollen tube) which can grow to the neighbourhood of the egg, carrying the male gametes with it. A further feature is the closure of the leaf (carpel) round the megasporangia (ovules). This is not found in the most primitive existing seed plants, such as the pines and firs, which are called " Gymnosperms," 1 because their ovules are not enclosed in carpels.

The pollen grains are brought to the ovule in Gymnosperms, and to a special receptive organ of the carpel (stigma) in most flowering plants, by the wind or by insects, and the perfection of the method of their transport has undoubtedly increased the chances of fertilisation in a great variety of species. From the stigma to the ovule the growth of the pollen tube and the life of the male gametes it encloses are protected by the fact that it takes place inside the tissue of the carpel. The female gamete is well protected by nucellus, ovule coats and carpel wall. All these arrangements enable the process of fertilisation to be carried out in flowers exposed to dry air in which exposed gametes would at once be killed.

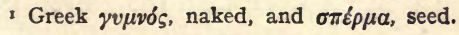


But the withdrawal of the process of fertilisation to the interior of the well-protected ovule has another great advantage-it enables the zygote, the result of fertilisation, to be developed into an embryo in a protected position, and to be supplied by the parent plant with the food necessary to its development in that position. In this way the young plant is able to establish itself in a short time after the seed germinates-it has a chance of penetrating quickly into the damp soil below the surface because it has a store of food at its disposal for rapid growth.

In the fern plant the sexual generation is a freeliving green plant produced by the germination of the spore on the soil. The young sporophyte is "parasitic" on the prothallus during the first stage of its growth. In the seed plant the prothallus is reduced to its lowest terms and is "parasitic" on, or rather in, the mother sporophyte (the " vegetative" plant). But not only so: the new sporophyte produced from the zygote is also parasitic on (i.e. it derives its food from) the mother sporophyte, and is protected by it in the altered megasporagium (seed) up to a certain stage of its development, when it can, on the germination of the seed, rapidly become free living.

Thus the seed plant has successfully solved the problem of sexual reproduction, and of giving the new generation a good start in life under conditions which the lower plants are quite unable to meet. The principle of the mating of the delicate and easily destroyed gametes inside the body of the parent, and of the protection of the embryo during the early stages of its life, is the same that we see successful in the higher animals, though the details of the mechanism are so widely different. Taken together with the power of maintain- 
ing and increasing the aerial shoot from year to year, thus permitting of that great increase in the bulk of the body which has given us the trees, the dominant plants over a large part of the earth's surface, the production of ovules and seeds has enabled the higher plants almost to cover the face of the globe.

\section{PRACTICAL WORK.}

Seeds and Seedlings.

A. The Broad Bean (Vicia Faba): a seed without endosperm and with hypogeal cotyledons.

(I) Make drawings of the side and front views of the seed showing the long scar of attachment to the pod and the position of the micropyle at one end of the scar. [By squeezing a bean which has been thoroughly soaked a drop of water can be forced out through the micropyle.] Pull the testa from a soaked bean to show the embryo, which completely fills the seed. Detach carefully one of the massive cotyledons, and draw the internal surface of the other with the curved epicotyl and the straight radicle at one side.

(2) Sketch a series of germinating seeds and seedling in different stages, marking cotyledons, epicotyl, foliage leaves (the lowest are rudimentary), axillary buds, terminal bud, primary root, lateral roots. The hypocotyl remains short.

B. The Vegetable Marrow (Cucurbita) : a seed with perisperm and thick epigeal cotyledons.

(3) Note the flatness of the seed, the scar of attachment (pitlike) and the adjacent micropyle. Split the seed lengthwise and observe the greenish layer of perisperm (nucellus), two rather thick cotyledons (though much thinner than in the bean), with very minute epicotyledonary bud between, and radicle.

(4) Make a series of sketches from stages illustrating the elongation of the hypocotyl, the pulling of the cotyledons from the testa, the straightening of the hypocotyl and the expansion and greening of the cotyledons. Mark the various organs of the seedling, the epicotyledonary leaves differing in shape from the cotyledons and the "peg" or "heel" at the base of the hypocotyl, which holds down one valve of the testa while the cotyledons are extracted. 
C. The Castor-Oil Plant (Ricinus): A seed with oily endosperm and epigeal cotyledons.

(5) Remove the mottled brittle testa (with its wart-like swelling at one end) from the seed, exposing the white mass of oily endosperm: split this open very carefully and observe the embryo in the middle. The very thin membranous cotyledons are extremely delicate and easily tear. They extend almost the whole length of the seed and have a complete system of veins already developed. Draw the embryo, noting the short cylindrical hypocotyl, continuous with the radicle, and the epicotyledonary bud.

(6) Sketch a series of stages in the development of the seedling, showing the gradual straightening and elongation of the hypocotyl (which may reach a length of 8 to $\mathrm{r} 2$ inches and a considerable thickness), the large flat cotyledons and the development of the epicotyledonary shoot. Note the buds in the axils of the coty?edons and the well-developed root system.

(7) If available, compare maize, wheat and date seedlings, showing endosperm and "sucking" organs : also the very small seedlings with epigeal cotyledons of some of our common weeds and garden crops. 


\section{CONCLUSION}

IN the foregoing chapters we have become acquainted with some facts about living beings, especially plants, and we may now briefly consider the chief lessons about life that we learn from them.

In the earlier chapters we saw that life everywhere depends upon a substance we call protoplasm-that this is present wherever we find life-in all organisms from amœba to man, and from bacteria to the seed plants-and that it is not present in lifeless objects. This protoplasm, as we saw, has a more or less definite chemical constitution. It is not itself a chemical compound, but a mixture made up in all organisms of the same classes of compounds. In physical structure, too, it is everywhere similar, belonging to a kind of substance-or rather a special condition of matter known as the colloid condition-which consists of very minute particles or droplets of one substance (disperse phase) dispersed through a continuous medium (continuous phase) of another. When the continuous phase of a colloid is a liquid such as water the colloid is called a sol, and certain sols as the result of the loss of water, or of other causes, pass into a jelly-like conditon known as a gel, in which the disperse particles are more aggregated, though there is no sharp limit between the sol and the gel conditions.

Protoplasm, as we saw, is a mixed colloid sol or 389 
gel, often fluctuating between the two conditions, with particles of protein, or loose combinations of proteins and fats or proteins and salts as the disperse phase, and water, with various substances in solution, as the continuous phase; and it is on this definite chemical nature and physical structure that the definite forms of activity-the "vital functions"-which are displayed by all living beings, depend. So much is certain, though we are still far from understanding in detail exactly how all the processes which take place in the protoplasmic sol or gel result in the characteristic life phenomena. In some cases we can already partly understand how this happens-as for instance in the case of the amœba taking certain objects into its body and casting out others (p. 69)-and as the study of the constitution and activity of protoplasm progresses we are steadily getting to understand more and more of these details.

Perhaps the two most fundamental properties or powers of protoplasm are first its power of maintaining the equilibrium of its physical structure within a certain range of external conditions (temperature, vapour, pressure, etc.), and secondly its power of assimilation, i.e. of taking into and making part of its own structure certain chemical substances that contain the elements of which protoplasm is itself composed. The first of these properties is the basis of the maintenance of an organism, of its continued life under varying conditions, provided these do not pass beyond certain limits; the second is the basis of growth and reproduction, of the increase in size and number of existing organisms. Neither of these powers is in itself unique in nature. There are many cases in which a portion of matter retains its equilibrium as a system for a longer or 
shorter time, and many others in which a portion of matter may be sald to assimilate and grow, for instance the simple case of a crystal growing in a solution of the salt of which it is composed by adding to its own structure molecules of the salt from the solution. But there is no other case in which so complicated and delicately adjusted a system as a unit of protoplasm (a free living cell) can do both these things indefinitely. It is the combination of complexity of structure and instability of its essential chemical constituents with the power of maintaining equilibrium of the system as a whole and of adding to the system by the assimilation of fresh material from outside that makes protoplasm a unique substance in nature, and what we call life a unique phenomenon.

To these two powers-of self-maintenance and of assimilation and growth-we must add the power of respiration, the power, that is, of oxidising organic substances, principally sugars, within the protoplasmic complex, and thus of setting free their potential energy, which is available for the production of mass movement and heat.

Though protoplasm has everywhere the same general chemical composition and physical structure, it differs in certain details in every different kind of animal and plant. If we accept the conclusion that all the phenomena of life depend upon the chemical and physical composition and structure of protoplasm, we must believe that the differences in the manifestations of life shown by different kinds of organisms depend on differences of composition and structure, just as the essential general uniformity of life depends on the essential general uniformity in the composition and structure of the protoplasm of all organisms. And this 
belief is obtaining more and more detailed support from recent research into the biochemistry of different species (p. 43).

The differences between closely allied but distinct kinds of animals and plants probably depend mainly on comparatively small differences in some of the proteins of which their protoplasm is composed, or on differences in the forms of aggregation of their protein molecules. When life first appeared upon the earth, or rather in the water, there were probably different kinds of very simple organisms having such differences, which affected their behaviour and consequently their mode of life. As to the details of such differences we know of course nothing, but it is evident that they must have gradually led to the adoption of such very different modes of life as we see to-day distinguishing, for instance, the animals from the plants. There are still existing many different kinds of minute unicellular organisms (Protista) which are neither unmistakable animals nor unmistakable plants, but which show a mixture of animal and plant characters. Yet the vast majority of organisms are either distinctively animals or distinctively plants.

The main characteristic of animals-on which all their other features are based-is that they can only take the nitrogen which all organisms must take in to make their proteins from ready made proteins, and since proteins are only found in nature in the bodies or as products of the bodies of organisms, animals must consequently feed on other organisms or on organic products. Plants, on the other hand, can take their nitrogen in simpler forms-as simple salts, or even in certain cases as free nitrogen. The colourless plants, which in the matter of nutrition are intermediate 
between animals on the one hand and green plants on the other, vary very much in respect of the form in which they can assimilate nitrogen. Most bacteria take it in complex compounds, but some in simpler ones, while others can fix free nitrogen. The fungi proper show a similar but more restricted variability. The green plants, on the whole, take their nitrogen in the form of simple salts, such as nitrates, but even they show variability. Some of the lowest forms can take their nitrogen as complex organic compounds, and even some of the seed plants (which as a class only use nitrates) can feed on organic nitrogenous compounds. This variability doubtless arose very early in the history of life, if it did not exist from the very beginning, and was the origin of the great differences of structure and mode of life in existing organisms.

The animals are the most specialised class. Organisms which can only take nitrogen in the form of proteins, and thus from organic sources, were forced, so to speak, into certain structures and habits, or they could not survive. They must either have a naked protoplasmic surface, as amœba has, through which solid food can be directly ingested, or alternatively they must have a special opening in the surface (mouth) through which food can be taken in. A further development, found in the great majority of animals is the existence of a cavity in the body $(g u t)$ into which the mouth leads and where the food is digested-broken up and rendered soluble - so that it can be absorbed by the living protoplasm; and an exit from this cavity (anus) through which the indigestible residue (faces) can be discharged. A still further development is a system of tubes (circulatory system, vascular system) which carries the soluble products of digestion to the living cells of 
all parts of the body where it is actually assimilated. A further consequence of the habit of living on solid organic food is that most animals-at least most of the higher animals-have to seek it, since it is very unevenly distributed through their surroundings, and thus have to move actively about, both for this purpose and in order to escape from being themselves devoured. They must move in definite directions and capture and devour their prey in definite ways. Therefore they must be sensitive to a great variety of external impressions of definite kinds, and hence the gradual development of the muscular system, the nervous system and the sense organs.

The higher animals are, in fact, brought into relation through their specific life-needs with a much greater range of their environment than is the case with plants, and this fact has called forth the very high differentiation and specialisation of their bodies. The necessities of locomotion have kept the bodies of active animals compact, with the different specialised organs and tissues very closely dependent on one another, so that no considerable part can be detached without crippling or killing the whole. This characteristic is known as integration (the making of a whole individual, the parts of which are closely interdependent) and is a feature of the highest (vertebrate) animals as well as of such highly developed specialised invertebrates as the insects and crustacea. Integration becomes greater and greater as we ascend the animal scale. The lower animals are much less integrated-the worms for instance, which can be cut into pieces without being killed-and when we come down to such forms as the hydroid polyps we find that they share many of the characters of plants. They are fixed branching forms of indefinite continuous 
growth, and little or no integration of the body as a whole, though they are true animals, living exclusively on organic food.

The powers of rapid locomotion and extreme sensitiveness to varied stimuli, together with the compactness and high integration of the body, are thus seen to be not necessarily associated with the fundamental animal character of living on solid organic food, but to be merely a possible development of animal organisation.

The early organisms which had the power of living on liquid foods, whether simple mineral salts and carbon dioxide dissolved in water, or more complex solutes such as sugars and nitrogenous substances derived from other organisms, were not forced to retain or develop the means of ingesting solid food, and it is these forms which have given rise to the class of organisms we call plants. Those which could intercept light energy by means of a pigment such as chlorophyll were able to use the simplest forms of raw food material, carbon dioxide and simple salts, and it is these forms which have given rise to the green plants-the main line of plant evolution.

The green plants, as the result of their greenness, have the unique power of building up protoplasm from simple inorganic constituents - they can assimilate carbon and nitrogen from carbon dioxide and nitrates. These they find everywhere around them, the latter in water and soil, the former in the air as well. Since they are able to find their food wherever there is enough water to dissolve it and to maintain their protoplasmic structure, they are the only possible forerunners of the extension of life upon the earth, living where other organisms cannot live, and themselves providing organic food for animals and for colourless plants. 
But the power which green plants have of constructing carbohydrates from carbon dioxide and water led, broadly speaking, to the formation of more carbohydrate material (sugars) within the cell than could be used for the formation of new protoplasm, since the available nitrogen in the form of nitrates, though widely distributed, is nothing like so abundant as the carbon dioxide and water. This excess carbohydrate material is condensed in the form of the polysaccharides starch and cellulose, the latter covering the protoplast in the form of the characteristic cell wall, which is such an important feature of the structure of plants and very largely determines the configuration and structure of their bodies and the direction of their evolution.

Under most conditions of life green plants make new protoplasm slowly, and their bodies contain a far smaller proportion of protoplasm and proteins, and a far larger proportion of carbohydrates, than those of animals. They grow slowly, continuously, and more or less indefinitely, and correspondingly their bodies are much less highly integrated than the bodies of the higher animals. An "individual plant" is much less of an "individual" than an individual higher animal, such as an insect or a vertebrate. Large parts of it can be cut off with little or no injury to the rest of the body, and any sufficient portion (in some cases very small fragments indeed) can grow into a new plant under suitable conditions.

These features are shared by the colourless plantsthe fungi-which have not the power of making carbohydrates from carbon dioxide and water. But fungi must live where they can obtain at least their carbohydrate food ready made, and thus they are not pioneers, like the green plants, but camp followers, 
like the animals. Their nutrition and metabolism are intermediate between the two, since they can use simpler nitrogenous foods than animals can, and though they grow much more quickly than green plants they tend, like the latter, to form an excess of carbohydrates. Some authorities believe that the fungi are all derived in evolution from green plants through the loss of chlorophyll, but we have no sufficient grounds for certainty. The fungi may have been derived, like the bacteria, directly from primitive forms which had a type of nutrition intermediate between that of animals and that of green plants.

We have thus seen that the starting points of the great primary differentiation of living beings into animals and plants must have depended upon differences in the constitution of the protoplasm of the earliest forms of life, just as the differences between species in existing organisms must ultimately depend upon differences between their protoplasms. Some organisms happened to produce the complex pigment chlorophyll (p. II2), which by the absorption of light enabled the protoplasm to use carbon dioxide as food-to assimilate carbon in that simple form. Such organisms differed from those which did not produce chlorophyll perhaps in no other respect, ${ }^{x}$ and yet so small a difference has eventually led, in the course of long ages, to the difference between a man and an oak tree. And this is the story of all evolutionary development. The widest differentiations have their origin in such small differences, of the order of specific differences, which are just sufficient to give the necessary bias to wholly

I We know existing unicellular organisms which differ from one another in just this way, and consequently, while almost identical in form and structure, have different habitats and modes of feeding. 
divergent lines of development; though the converse is by no means true-only a few specific differences actually lead to wide divergence in subsequent evolution. We are still very ignorant as to the causes of the first appearance of such small differences. From the point of view of their effects they are chance occurrences.

We have seen that the activities of living substance which are common to all organisms-the vital functions -are functions of the particular, the unique physicochemical complex which we call protoplasm, and that we can partly explain how these functions or activities result from the physico-chemical structure. We have also seen reason to believe that the great primary differentiation between animals and plants arose from small differences in the protoplasm of different primitive organisms, and that similar differences exist now between closely allied species. There is evidence that such differences are still frequently occurring between different individuals or groups of individuals of the same species, and that in this way new species originate. In the higher forms of life, animals and plants with complicated bodies of definite structure, such slight changes in the chemical composition of the protoplasm may be expressed in various definite ways, in slight but definite alterations in the form and structure, or in the colour, of the body or of particular parts of the body, such as we know is actually characteristic of the differences between species. In some cases such changes, leading to the origin of new species, can be shown with fair probability to be the result of the effect of some change in the surroundings, for instance increased dryness or wetness, or the presence of some chemical substance which is absorbed by the body. But in the greater number they are probably the result 
of changes in the protoplasm due to internal causes which we cannot yet trace.

Another and probably very important cause of the appearance of new species is the crossing of distinct but closely allied species, the gametes contributing to the zygote protoplasm (chromatin) of slightly different chemical composition or physico-chemical structure, and thus producing an individual of a new type.

Not all new forms which come into existence in one of these ways succeed in surviving and maintaining themselves. Some of the changes that may occur may make the protoplasmic "machine" unworkable, and in that case the new organism dies and no new species appears. In other cases the change may lead to a modification of structure or function which is badly out of harmony with the conditions in which the new organism finds itself. For instance, to take a purely hypothetical case, a seed plant living in a climate where it was occasionally exposed to very dry air might undergo a protoplasmic change which led to the cells of the epidermis being unable to form a good cuticle, so that the plant would rapidly lose water by evaporation from its epidermal cells. Such a plant would dry up and die, and the new form would never establish itself.

But in some cases the change may actually lead to the new form being better adapted to the life conditions, and in that case the new form will not only survive, but will have an advantage in the struggle for existence. We saw in the last chapter that a very small proportion of the seeds produced by a seed plant actually find suitable conditions for germination, and that a very small proportion of the seedlings produced by the seeds which do germinate grow into adult plants which 
themselves set seed. It is clear that those which are best equipped for succeeding under the existing conditions will be most likely to survive in the struggle to establish themselves. For instance, suppose the place where the seeds fall to the ground is an open spot where heavy rain falls at intervals of several weeks, but that there are very dry periods between: suppose further that the surface layers of soil, say to a depth of 2 or 3 inches, are very permeable to water and easily dry by evaporation, but that the deeper layers are permanently moist. If the radicles of seeds germinating during the rain period grow slowly, so that they have not penetrated more than an inch when the dry period begins, the root will be starved of water and the seedling will die. But if a change occurs in the constitution of the seedling so that the radicle grows quicker and the root gets down to the permanently moist layers of soil before the dry period sets in, the seedlings will establish themselves and grow into adult plants.

To take another case: suppose the seeds to be those of a plant growing on the floor of a forest where the soil is at all times moist, but the light penetrating the foliage of the trees is not more than sufficient for the needs of the seedlings. Suppose the seeds of various species fall on the soil in great numbers, and, the conditions for germination being favourable, nearly all germinate. Here there is no difficulty about the roots-there is plenty of moisture in the soil for all. But the seedlings will grow up very crowded and will cut off the light from one another. Those which grow most rapidly will rise above the others, will get all the available light, and will establish themselves; the slower growing seedlings will be badly shaded and 
will eventually die. Any change in the constitution of the seedling of a species under these conditions which enables its shoot to grow more quickly will give it a decisive advantage in the struggle for light.

In the former case the seedlings were struggling with their inorganic environment, in the latter competing with other organisms. The world of organisms is the scene of both these kinds of struggle at every stage of the life history of individual species, but the struggle is most severe in the early stages of life, when the individuals are most numerous and have not yet established themselves. This struggle for existence necessarily leads to the success of the forms which are best equipped to succeed. There is a selection of such forms by the elimination of those which do not succeed, and it is this which Darwin called natural selection.

One of the first things that must strike the student of biology is the marvellous adaptation of the different kinds of plants and animals to the conditions in which they live, and this adaptation is in large part brought about by the success of those forms which have changed in directions advantageous to them. Since they have succeeded they are the forms we actually meet withthe failures have disappeared. But there are many degrees of success. Some species are tremendously successful, spreading widely and constantly establishing themselves in new places-carrying, so to speak, all before them. Others hold their own, but no more, succeeding perhaps in some places, failing in others. Again, there are species which are not now holding their own, but are slowly, or rapidly, dying out.

In the course of evolution different species have become closely adapted to different kinds of life conditions-habitats as they are called-for instance hot 
moist climates, hot dry climates, cold climates, and so on; and again to seashores. marshes, forests, as weeds of cultivated land, and to many other special habitats. They continue to grow in these places and no others, because their economy is adjusted to these particular conditions of life.

In all this we see varied special cases of the great universal law of equilibrium, which governs all the processes of which we have any knowledge, from the movements of the planets to those of molecules, atoms and electrons, from the activity of protoplasm to the vagaries of the human mind. All things which exist are constantly tending towards positions of balance or equilibrium, i.e. of relative repose, and all activity, all motion, represents some phase of this universal process. The universe consists of the most varied kinds of systems in relatively stable or unstable equilibrium, and every fresh disturbance of equilibrium from outside any system leads to fresh activity in the system which tends towards the establishment of a new equilibrium.

A free-living unit of protoplasm represents a very striking special case of a system of particles which can maintain a moving equilibrium, by assimilation of fresh particles of special kinds of matter from without, and the breaking down (katabolism, respiration) of certain substances within. This double process it can only maintain within a certain range of external conditions. Outside these the equilibrium is destroyed and the organism dies, the matter of which it is composed breaking up into simpler systems of dead matter which return to simpler states of equilibrium. By assimilating faster than it katabolises the protoplasmic unit 
grows, till its equilibrium can no longer be maintained as a single system, and it divides, breaking up into fresh units like itself. When these units separate reproduction takes place, when they remain together a multicellular body arises. The different cells of this, because they are differently placed in regard to each other and to the environment, are differently affected by each other and by the environment, and differentiation begins between the different cells. A new equilibrium tends to be established, and we have a characteristic form and structure developed. This must be in general harmony with (i.e. in equilibrium with) the external conditions, or the organism will die and disappear. Only those forms which are in harmony with their surroundings will survive, and it is only those which we see around us. But the possible kinds of harmony are very varied, for in the first place the protoplasm of different kinds of organism is different, owing to the perpetual changes which go on in the protein complexes that form the basis of protoplasm, the perpetual readjustments of internal equilibrium; and in the second place the external conditions are different for different individuals. The constant interactions of one and the other are as constantly bringing about fresh adjustments, new positions of the total equilibrium of the whole organism -in other words, different species of organisms.

These are the causes of the immense variety in the forms and structures of the organisms that we see around us, and of the fact that they are in general, and very often in the minutest particulars, wonderfully well adapted to their surroundings. Otherwise they could not come into existence. But alongside of, coexisting with, the features of structure and function that are thus adapted, we see many others which are 
not. There is no bar to the appearance of characters which are of no use to the organism, nor even of characters which are disadvantageous to it, provided they do not handicap the organism sufficiently to destroy its chances of continued existence.

Proof that a structural or functional character is of use to an organism is no explanation of the origin of the character in question. A useful character, even when the organism could not survive without it, is merely an expression of a partial equilibrium with the environment. The origin, that is the causation of a character, is not to be sought along these lines, but by studying the play of forces at work. We have considered several cases, for instance the origin of the sexual differentiation of algal gametes (pp. 200-202), the causes or the differentiation between the external and internal cells in the Brown Seaweeds (p. 228), the ability of the fruits or seeds of many seashore and riverside plants to float in water for a long time without injury (p. 372), in which it is clear that the causation of these characters is distinct from their advantageousness to their possessors. Their usefulness may explain their maintenance, but not their origin.

All characters alike, essential, useful, useless, or harmful, are the inevitable products of an organism's constitution-in the last analysis of the constitution of its protoplasm-reacting according to the laws of physics and chemistry to the conditions of its environment. The science of biology, like every other science, is concerned with causation, the fixed relations between phenomena, and it is only by the study of the causation of the characters of living organisms that our knowledge of biology can be advanced. 


\section{INDEX}

achromatic spindle, 104-106, 278 adaptation, 401 adsorption, 53 adventitious roots, 26I, 288, 235 æcidiospores, I 79-1 81

aerial (subaerial) shoot, 255-26I, $263,264,266$ aerobic respiration, $132,135,150$ aggregation of protein molecules, 63

albuminous seed, 375

alcoholic fermentation, 132-136

anabolism, 79

anaerobic respiration, $132,135,150$ Algæ, 29, 72, 73, 96, 184, 203 , $206,207,214$

alternate hosts, $18 \mathrm{I}$

amino-acids, 122

A maeba, 64-72, 74, 79, 81, 83, 84, $92,96,393$

andræcium, 345

Angiosperms, 28

animals and plants, differences between, 22-26, 206, 207, 392397

annual plant, 256,257

annual rings, 335,336

anther, 346, 347, 351, 355-357

antheridium, 223, 224, 234-236, $244,245,250,25 \mathrm{I}$

antipodal cells, $349,35^{\circ}$

apical cell (of Fucus), 219

apical (primary) meristem, 289, $291,297,320,363,377$

apocarpous, 352

archegonium, 235, 236, 244, 245, 250,251

" aromatic substances," 44

assimilation, 69, 78, 390, 402

autumn wood, 335,336

axil, 258

axillary bud, 258, 259, 262-265, $322,323,326,330$ bacillus, I 39,148, I 49, I $54^{-1} 5^{6}$

Bacteria, 30, 51, I I $3,127,136$, 138-156, 393

bacteriology, 148

bark, 299, 329, 340, 341

binary fission, 72,88

bone, 98

Bordeaux mixture, 178

bordered pits, $276-278$

bracts, 345

brewing, I 32-1 34

Brownian movement, 50, 62

Bryophytes, 29

bud scale scars, 330,342

bud scales, 329, 330, 342

buds, 256-259, 261-266, 316, 322, 223, 326, 328-330, 378, 381

bulb scales, 265

bulbils, 265

bulbs, 265, 266

callose, 274

calyx, $345,353,364$

cambium, 298, 325, 328, 331, 336

carbohydrates, 37-41, 81, 90, 92, $120,122,152,154,159,220$, $274,278,285,375,377,380$, 396,397

carotin, I 1 2, I86, 214

carpels, 346, 352, 353, 354, 355 , $365-368,369$

cartilage, 95, 98

catalysts, 44

cell, $31,32,54,56,58,66,80$, 92-109, 39 I

cell doctrine, 96-98

cell sap, 101, 107, 109, III, II8, 290,296

cell wall, $31,72,73,98,99-101$, 396

cellular structure, 31

cellulose, 41, 45, 47, 92, 96, 98, $107,122,128,136,141,152$, 
I 54, I 86, 270, 275, 276, 278$281,284,377,380$

cereals, $38 \mathrm{I}$

chemosynthesis, I I 5

chemotaxis, 87

chemotropism, 35I

Chlamydomonas, 24, 87, 185-192, 194, 195, 201, 203

chlamydospores, 162

chlorophyll, 23, 32, 112-114, I I 7, I 78, 21 4, 302, 317, 395, 397

chloroplasts, $32,72,73,74,80$, IO2, I12, I I 3, I I 5-I 20, I 85, $190,233,234,236,242,302$, 303

chromatin, 66-68, 103, 106, 128, I 29, I 40, 399

chromosomes, 104-106

cilia, $85,93,141$

classification, 26-28

Clubmosses, 29, 248, 249

coagulation of proteins, 62

coccus, I $39, I_{4} 8, I_{54}$

cœnobium, 192-199

coffee disease, $\mathbf{r} 8 \mathbf{r}$

collenchyma, 279, $311,317,320$

colloids, $48-60,61,62,141,389$

companion cells, $272,273,275,293$. 324,332

competition, 384,401

conceptacle, $216,222-225$

conducting cells (of Fucus), 222

conidia, 164, 173, 176, I 77

connective tissue, 95,98

continuous phase, $49,61,390$

contractile vacuole, 65,84, I 86

cork, 284, 299, 331, 337-342

corms, 263, 264, 265, 266, 325

corolla, 345

cortex, 29I-293, 296, 297, 30I

$317,320,321,322,326$

cortex (of Fucus), $217,220,222$

cotyledons, $256,257,362,363$, $378-380$

crystalloids, $49,5 \mathrm{I}, 55$

cuticle, II $, 242,284,303,307$, 308

cutin, 284, 293, 294, 297, 303, 305

cytase, $45,272,377,380$

cytoplasm, 66, 67, 73, 74, 93, 96-

98, 100, I0I, 107, 108, I 1 2, I I5,

I 27. I 58, I61, 164, I 85, 186,

I89, 190, 200, 202, 207-209,

$217,218,223,236,270,274$,

$276,278,286,348,350,376$
" damping-off," I 72-3

death rate of seedlings, $38_{3}$

dialysis, 55

diastase, $45,120,272,377,380$

differentiation, $80,93,94,215,238$

differentiation, intracellular, 81 , 93

diffusion, 54, 55, 99, I 14, I I6, I I 8, 222,339

disease bacteria, I $54-1566$

disperse phase, $49,55,61,62,390$

dormant buds, 330

ectoplasm, 62, I01, I02

egg apparatus, $35^{\circ}$

elaters, 235, 237, 240, 279

embryo, 237, 240, 244, 245, 25I, $255,288,361,362,363,364$, $374-377,386-388$

embryonal cell, 361,362

embryonic cells, 100-102, 270, 320 emulsoid, 49

endodermis, 291, 293, 294, 296, $297,317-319$

endoplasm, 62, 102

endosperm, 349, 352, 361, 362, $364,374-380,388$

endospermic seed, $375,379,380$

energid, 97, 98

energy, $25,26,38,42,77,81,82$, $84,85, \mathrm{I}_{2} \mathrm{I}, \mathrm{I} 30, \mathrm{I}_{32}, \mathrm{I}_{42}, 38 \mathrm{I}$ enzymes, $44-46,67,94,120,132$, I50, 272, 274, 377

epicotyl, $25^{8}, 362,37^{8-380}$

epidermis, I I $5,242,272,273,275$, $278,284,302,303,305-307$, 309, 310, 316, 321, 338, 339

epigeal cotyledons, $378,379,387$, 388

epigynous, 353,354

equilibrium, $55,57,108,404$

equilibrium, law of, $55,402,403$

ethyl alcohol, 132

etiolation, 316

Eudorina, 194, 195

exalbuminous seed, 375

eyespot, I 86

facultative gametes, 204

facultative parasites, I 58, I69

families of plants, 27

fat-cells, 95

fats, 41, 42, 6r, 63, 77, 91, 95, I 20, I 54, 376, 377, 381, 382, $390,392,396$ 
feeding, $68,77,78$

female gamete, $19 \mathrm{I}, 195,200,202$, $210,344,350-352$

fermentation, 130, 132-136, 138, I5O, 151

Ferns, 29, 244-248

fibres, 2 I $8,279,280,3 \mathrm{II}$

filament, 346

filamentous green algæ, 203

flagella, 85 , I 4 I, 172, I86, I92, 193 , $198-200,235,236,244,245$

floral leaves, $258,344-346,352-$ 354

flower, $258,344-360$

flower bud, $257,25^{8}$

Flowering Plants, 28

foliage leaf, 22, 242, 244, 246, 256, 302-314, 31 5, 325

food vacuoles, 69

foot, $237,244,245$

formaldehyde, II 4

frond, $215,216,220$

fruit, $258,361-374$

fruit buds, 329

fucoxanthin, $2 \mathrm{I}_{4}$

Fucus, 214-230, 240, 351

Fungi, 30, 96, 127, 157-183, 231,393

gametes, 188-192, 195, 198, 204, $205,209,222,234,239,250$, $252,344,349,350,35^{2}$

gelatine, $54,59,285$

gel membrane, 54-56, 58, 62, 66, IOI, $\mathrm{IO}_{3}$

gels, 53, 54, 58, 59, 62, 63, 66, 103, 285

genera, $27,139,148$

geotropism, $87,288,315$

germ cells, 185, 197, 198, 206, 207, 267

gland cells, 94, 95, 272

glucose, 38, 39, 8I, I I 4, 120

glycogen, 26, I 29

growth, $79,87,88,90,106,160$, 390

guard cells, 242, 303, 305-308

Gymnusperms, 28, 385

gynæcium, 346, 353, 354

habitats, 4 or

hæmoglobin, 43, 82

haustoria, 300, 372

heartwood, 337

herbaceous plants, 255-258, 267, 328,329 heterogametes, 190

heterospory, 249-252

holdfast, 215, 219

holophytic, 78

holozoic, 78

Horsetails, 29, 249

humus, $125,152,166$

hydrotropism, 87, 289

hyphæ, 157, 164

hypocotyl, 256, 257, 362, 378-380

hypogeal cotyledons, $379,380,387$

hypogynous, 353,354

"immortality" of unicellular organisms, 184,185

inferior ovary, $353,354,368$

inflorescence, 329,345

integration, 394,395

intercellular spaces, 99, 115,285 , 293, 304, 309, 310, 335, 339

intercellular substance, 98

" internal atmosphere," II 5, 285

internodes, 258, 316, 345

invertase, 45

irreversible gel, 62

isogametes, 190, 204

karyokinesis, 103-106, 209

katabolism, 79, 83, 84, 402

kinetic energy, $25,38,8 \mathrm{I}-83,85$, I 2 I

lævułose, 38, 39, 45

lenticels, $339,34 \mathrm{I}, 343$

leucoplasts, I 19

Lichens, 30

lignification, 283,284

lignin, 283

lignocellulose, 283

lipases, 45,377

liver, 26,83

Liverworts, 29, 232-238

Lycopods, 248,249

male gametes, 191, 195, 200, 202, $210,250,252,344,347,350$, $35^{2}$

maltase, 45 , 120

malting, $133,38 \mathrm{r}$

maltose, 38,45

mechanical tissue, 278, 279, 312, 320

medulla, 217

megasporangium, 249-252, 254 , $255,344,345,347,348,385$ 
megaspore, 249-252, 344, 348-350, 384,385

meristem, 100, 102, 258, 270, 289, $320,321,377$

meristem, secondary, 298, 299, $328,330,331$

meristematic cells, 101-103, I27, $320,32 \mathrm{I}$

mesophyll, I15-117, 242, 270, $302-305,307,309-311,313$

metabolism, 62, 65, I 22

metabolites, 62

metaphloem, 324

metaxylem, 295, 296, 324

micropyle, $348,349,350,352,378$, 387

microsporangium, 249-252, 344, 345-347

microspore, $249-252,344,346$, $347,348,384,385$

middle lamella, 107, 276, 278, 285,338

midrib, $215,233,234,310$

Mosses, 29, 232, 238-241

movement, $22-24,68,84-87,93$

Mucor, I 58-164

multicellular organism, $66,80,93$, $96,206,403$

muscle tissue, $94,95,98$

must, 134

mycelium, I 57,164

natural selection, 401

nectary, $272,356,357$

nitrification, 153

nodes, $258,316,345$

nomenclature, 27

non-cellular plants, 97, I 58

non-endospermic seed, 375,380

nucellus, 348, 349, 364, 385

nuclear membrane, 66, 103, I04, Io6

nucleolus, I00, $\mathrm{IO}_{3}$

nucleus, $62,66-68,73,95,97$, 100, 102, 103-106, 107, II7, I25, I 39, I $58,162,185,187$, 189, 191, 20I, 207, 217,2 I , 223-225, 226, 236, 272-274, $347-350,352,36 \mathrm{I}$

obligate parasite, 157 oils, $42,376,381,382$ oogonium, 223

"organic " compounds, $36,3^{8}$ osmosis, 57, 3 I I osmotic pressure, $57, \mathbf{1 0 8}, \mathbf{1 3} \mathbf{1}, 305$ osmotic substances, 57,108

ovary, 348, 351, 353, 354, 365368

ovule, $254,255,348,349,359,360$, $36 \mathrm{I}$

palisade layer (of Fucus), 217 , 222

Pandorina, 192-194

pappus, $370,374,383$

parasites, 78, I 54, I 58, I 69-1 83 , 300

parasitic, $23,78,157,300$

parenchyma, 270,271

parthenogenesis, $I_{72}$

pectin, 285

pedicel, 345

peduncle, 345

Pellia, 232-238

Pellionia, I26, 234

Penicillium, 164-166

pentosans, 275,285

perennation, 265

perennial, 256

perianth, 345

pericarp, 364, 365, 368, 369-37 r

pericycle, 293, 317, 318, 320, 338, $34^{\circ}$

perigynous, $353,354,366$

perisperm, 364, 387

petals, 345, $346,353,355$

phroplasts, $214,217,218,223$, 225,228

phellogen, 299, 331, 337-341

phloem, 292, 293, 295, 297-299, $310,317,318$

phloem fibres, $332,334,336,337$

photolysis, I I 5, 285

photosynthesis, 32, 113-115, I I 8 , I $21,220,302,303,309$

phototaxis, 87

phototropism, 87, 160, 289, 315

Phytophthora, 175-177

piliferous layer, 290, 292, 297

pith, 295, 317, 319, 321

pits, I08, 276-278, 279-282

placenta, 348, 349, 355, 368

placentation, 355,368

plankton, I 84

plant pathology, I $8 \mathrm{r}$

plasmolysis, I09, I I I , 2 I 3

plastids, 102, 108, $119,127,270$

Pleodornia, 195-197, 206

pollen, $347 ; 355^{-35^{8}}$ 
pollen grains, 346-348, 351, 355, 358,385

pollen sac, $346,347,351$

pollen tube, $347,349,350,351$, 385

pollination, $35 \mathrm{I}, 354$

pollination, cross, $351,355,35^{8}$

pollination, self, $35 \mathrm{I}, 35^{8}$

polysaccharides, $\mathbf{4 0 , 4 1}, 49,396$

Potato blight, I $75^{-1} 77$

potential energy, $25,38,42,81$, 85,121

principal rays, 335,336

protandrous, 356, 357

proteans, $45,272,377$

protein cells, 272,274

proteins, 42-44, 49, 61-63, 73, 77, $122,130,142,151,154,272$, $278,375,377,381,390,392$

prothallus, $244,245,24^{8-25}$ I

Protista, 30, 392

Protococcus, 72-74, 80, 92, 93

protophloem, 324, 332

protoplasm, 31, 32, 33, 36, 56, 58 , $6 \mathrm{r}-64,64-70,76,77,82,84$, IOO-IOI, 389, 404

protonema, 240

protoxylem, 295, 296, 297, 322325

pseudopodium, 62, 65, 68, 7I

Pteridophytes, 29, 243-251, 384

Puccinia, 178-181

pulses, 381

pure culture, 147,148

"pure strains," 67, 149

putrefaction, I $5 \mathrm{I}$

pyrenoid, 72, 73, 185, 186, 190, 207, 208, 209

Pythium, 172-1 75

rays, 295

receptacle, $345,353,354,365-368$

reproduction, $71,73,87,184$

respiration, $39,81-83,118,121$,

$132,135,136,150,159,289$,

$305,376,377,391$

reversible gel, 54,62

rhizoids, 221, 225, 233, 238, 239, $241,244,245$

rhizomes, $257,259-262,265,266$, 381

ringworm, 169

root cap, 285, 289, 290-292

root hairs, 24I, 289, 290, 294, 296

root, the, 24I, 288-301 runners, 26I

Rust of wheat, 178-18I

Saccharomyces, 128,136

salts, $73,77,123,130,142,152$, I 59, 186, $217,220,240,241$, $290,296,300,304,311,392$, 393,395

Saprolegnia, I7 7,172

saprophytes, 127, 157-168

saprophytic, $78,157,158$

sapwood, 337

scale leaves, $259,262,325$

secondary meristem, $298,328,331$

secondary rays, $334-336$

secondary thickening, 255,298 , 328

secondary tissue, 298, 329

secretory cells, 272

seed, 254, 255, 258, 361-364, $365,368,369-372,375-388$

Seed Plants, $28,243,252,254,255$, $344,384-386$

semipermeable membrane, 56,62 , 108, 109

sepals, 345, 346, 353

sexual differentiation, 184, 190, 200, 205, 210211,234

sieve plate, 274

sieve tubes, 229, 274, 293, 297. $311,324,332,333,337$

sol, $48-50,53,54-56,61-63$, го , 389

solutes, 48, 55-57, 6r, 108, 293 , 296, 299

solution, colloid, 48

solution, crystalloid, $48,55,59,39$ I

solution, "true," 48,59

solvents, 48, 55, 56

soma, 184, 185, 195, 198, 205, 206 species, $27,42,43,67,148,149$, $398,401,403$

spectrum (of chlorophyll), I 13

spermophytes, see seed plants

sperms (= male gametes) 198, 199-

20I, 212, 223-225, 230, 235 .

$236,244,245,248,250,25 I$

spirillum, 139, 148

spirochæte, 148, 154, 155

sporangiophore, 160

sporangium, $160,16 \mathrm{I}$

spore capsule, 237,240

spores, I $31,1_{43}-I_{45}, 1_{4} 6, I_{5}$ I,

$155,160-162$

sporidia, 179,180 
sporogonium, 237,240

spring wood, 335,336

stamens, 345, 346, 352, 353, 364

starch, 40, 43, 45, 77, 90, I I 8-1 20,

I 22, I 78, I86, 209, 376, 377, 381,396

starch grains, 40, 46, I1 7, I I 8, 126, 234, 301, 319, 375, 380

sterilisation, 146,147

stigma, 348, 35r, 355, 356, 357, 364,385

stimulus, 26, 86

stipe, 215, 216, 219

stolons, 261

stoma, II5, 242, 303, 305-308, 31 7, 339

stone cells, 280

storage tissue (of Fucus), 222

struggle for existence, 4 or

style, $346,348,351,364,370$

subaerial shoot, see aerial

suberin, 284, 299, 339

subordinate rays, 335,336

sucrose, $38,39,45$

sugars, 38, $39,45,56,57,77,81-$

$83, x_{4}, x_{1}$ - I 22, I 49, I 59, 305,

$310,380,395,396$

supporting tissue, 279,320

surface energy, 52

surface tension, 52, 69, 70

suspensoid, 49, 59

suspensor, $36 \mathrm{r}-364$

synergidæ, $349,35^{\circ}$

taproot, 256, 257, 267, 288

taxis, 87

teleutospores, I 79-181

terminal bud, $256,258,261,262$, $265,316,320,321,328$

testa, 364, 369, 37 r, 378-38o

thallus, 214,233

tissue mother cell, 33I-334

tissues, $80,94,95,98,106,107$. $217,238,241,242,270$

tracheids, $280-283$

tracheids, annular, 28r, 295

tracheids, pitted, 282

tracheids, reticulate, 282

tracheids, scalariform, 282

tracheids, spiral, 28o-282, 295

transpiration, 304, 310

tropisms, $87,288,315$

tubers, I I9, 262, 265, 266, 325

turgor (turgidity), 108, 109, I I0, 305,307 ultra-microscope, 50

ultra-microscopic particles, 50, 5I, $6 \mathrm{r}$

unicellular organism, 66, 93, 96, I 84,185

urea, 65,83

uredospores, r78-r 81

uric acid, 83

vacuole, 62, 100, 101, 107-109,

I I I, I I 5, I I 7, I 25, I 28,158 ,

$207,217,218,270,273,274$,

275,296

vacuole wall, 101, 102

vascular bundles, 302, 310, 317 ,

$318,321,324,325$

vascular cylinder, 29I, 293, 294, 295, 297, 317, 318, 319, 322, 324,325

Vascular Plants, 24 I-243

vascular system, 242, 246

vascular tissue, 317

vegetative reproduction, 206, 241 , $248,266,267$

veins, $302,310-312,325$

vessels, 282, 283, 293, 296, 297 . $324,325,332,335$

vestibule, 306,308

"vital functions," $76,92,97$. 390

Volvox, 197-200

water cultures, I 23

water, essential importance of, 37

water tissue, $275,276,310$

wilting, 109, 308, 320

wind pollination, $35^{8}$

wine-making, I34

wings, $215,216,218$

winter buds, 329,330

woody plants, 255,328

wort, 132-134, 381

xanthophyll, I I2, 2 I 4

xylem, 293, 295, 298, 302, 304, $310,311,317,318,331-334$

xylem (wood) fibre, $332-335$

yeast plant, I 28-136, I 42, I43, I50

zooglœa, I4 I

zoospores, I 72, I 73, I 75, I 76, 203 zygote, I62-I $64,175,189,208-$ $210,225,23^{6}, 244,35^{2}, 36$ I 
Printed in Great Britain by UNWIN BROTHERS, LIMITED

LONDON AND WOKING 


